\title{
Composting of Residuals from a Strawboard Manufacturing Facility
}

\author{
by
}

\section{Scott Douglas Chapman}

\author{
A Thesis Submitted to the Faculty of Graduate Studies of \\ The University of Manitoba \\ in Partial Fulfillment of the Requirements of the Degree of
}

MASTER OF SCIENCE

\author{
Environmental Engineering Program \\ Department of Civil Engineering \\ University of Manitoba \\ Winnipeg, Manitoba R3T 5V6 \\ Canada
}

Copyright $@ 2006$ by Scott Douglas Chapman 
THE UNIVERSITY OF MANITOBA

FACULTY OF GRADUATE STUDIES

COPYRIGHT PERMISSION

Composting of Residuals from a Strawboard Manufacturing Facility

by

Scott Douglas Chapman

\begin{abstract}
A Thesis/Practicum submitted to the Faculty of Graduate Studies of The University of Manitoba in partial fulfillment of the requirement of the degree

of

Master of Science
\end{abstract}

Scott Douglas Chapman (C) 2006

Permission has been granted to the Library of the University of Manitoba to lend or sell copies of this thesis/practicum, to the National Library of Canada to microfilm this thesis and to lend or sell copies of the film, and to University Microfilms Inc. to publish an abstract of this thesis/practicum.

This reproduction or copy of this thesis has been made available by authority of the copyright owner solely for the purpose of private study and research, and may only be reproduced and copied as permitted by copyright laws or with express written authorization from the copyright owner. 


\begin{abstract}
Dow BioProducts Ltd. operates a strawboard manufacturing facility located near Elie, Manitoba that has an annual waste stream composed of approximately 6,000 wet tonnes (wt) of straw too wet to use in the process and 5,000 wt of fine process residuals (unders). Composting is a viable alternative for managing this waste stream although limited research has been completed on effectively composting a high carbon to nitrogen ratio material such as straw.
\end{abstract}

To determine optimal compost recipe formulation, Phase 1 of this study utilized benchscale reactors to compost various recipes using straw, process unders, and a lagoon mixture (nitrogen amendment) as feedstock materials at different moisture levels for a nine-week period. It was concluded that water addition and the addition of unders to the compost mixture increased degradability, while the added nitrogen source of a lagoon mixture did little to enhance degradability.

Due to changes in the strawboard manufacturing facility's waste stream and the results obtained in Phase 1 of the study, Phase 2 focused solely on the straw feedstock and investigated the effects of straw particle size using four unshredded/shredded straw combinations (by volume): (1) all unshredded straw, (2) 2/3 unshredded straw, 1/3 shredded straw, (3) 1/3 unshredded straw, 2/3 shredded straw, and (4) all shredded straw. The recipes were composted in 140L rigid plastic containers for a period of 180 days at a moisture content of $70 \%$ (wet basis). The rate and degree of straw degradation was assessed by measurements of volume, volatile solids (VS), and lignocellulose fibre (lignin, cellulose, and hemicellulose). It was found that VS degradation was greatest for recipes containing both shredded and unshredded straw, with the recipe containing $2 / 3$ shredded straw performing the best (35\% VS removal). Pure unshredded and shredded 
straw had VS reductions of $29 \%$ and $26 \%$, respectively. Total volume reductions ranged from 80 to $90 \%$. Hemicellulose was completely degraded for all recipes by day 95 . By day 180 cellulose content had decreased from $3.75 \mathrm{~g} / \mathrm{g}$ ash to $0.75 \mathrm{~g} / \mathrm{g}$ ash for all four recipes. By day 180 , lignin degradation was greatest for recipes containing both shredded and unshredded straw (reduction from $1.0 \mathrm{~g} / \mathrm{g}$ ash to $0.4 \mathrm{~g} / \mathrm{g}$ ash) while lignin content decreased from $1.0 \mathrm{~g} / \mathrm{g}$ ash to $0.6 \mathrm{~g} / \mathrm{g}$ ash for the other recipes. Based on the experimental results it was concluded that recipes containing a mixture of shredded and unshredded straw provided better composting conditions. These two recipes had both greater overall removals and greater removal rates of volatile solids, cellulose, and lignin. 
TABLE OF CONTENTS

SECTION

PAGE

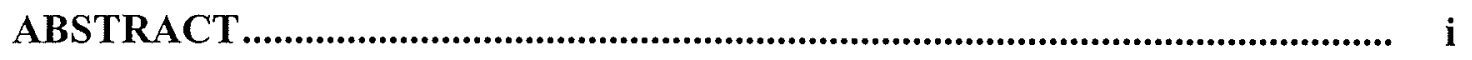

TABLE OF CONTENTS ….......................................................................... i

LIST OF TABLES ............................................................................................. v

LIST OF FIGURES ........................................................................................

LIST OF EQUATIONS.................................................................................... vi

NOMINCLATURE AND ABBREVIATIONS................................................... vii

ACKNOWLEDGEMENTS ................................................................................. viii

$1.0 \quad$ INTRODUCTION..................................................................................... 1

$2.0 \quad$ LITERATURE REVIEW ................................................................. 3

3.0 OBJECTIVES …............................................................................ 5

4.0 MATERIALS AND METHODS .................................................... 7

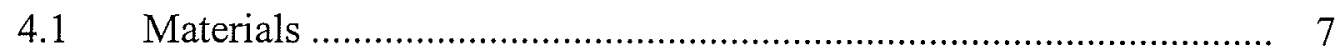

4.2 Composting Set-Up.................................................................. 9

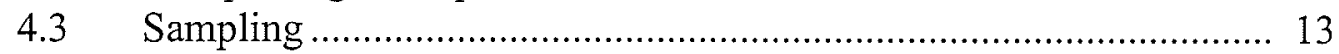

4.3 Physical and Chemical Analyses ...................................................... 14

$5.0 \quad$ RESULTS AND DISCUSSION ......................................................... 16

5.1 Phase 1 Composting...................................................................... 16

5.2 Phase 2 Composting.................................................................. 19

5.2.1 Particle Size, Mass, Volume and C:N Ratio ......................... 19

5.2.2 Temperature and Oxygen.................................................. 22

5.2.3 Volatile Solids Reduction ................................................. 24

5.2.4 Fibre Degradation ........................................................... 26 
6.0 SUMMARY AND CONCLUSIONS .................................................. 31

7.0 RECOMMENDATIONS AND ENGINEERING SIGNIFICANCE ...... 33

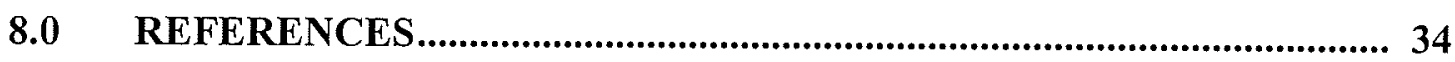

APPENDIX A - PHASE 1 COMPOSTING PHOTOGRAPHS APPENDIX B - PHASE 2 COMPOSTING PHOTOGRAPHS APPENDIX C - PHASE 1 EXPERIMENTAL LABORATORY DATA APPENDIX D - PHASE 2 EXPERIMENTAL LABORATORY DATA 


\section{LIST OF TABLES}

Table 4.1: Phase 1 Small Bench-scale Reactor Recipe Descriptions ..................... 9

Table 4.2: Phase 1 Recipes Used for Comparisons ......................................... 9

Table 4.3: Masses of Unshredded and Shredded Straw Used in Phase 2 Recipes .... 13

Table 5.1: Phase 1 \& 2 Feedstock and Initial Bench-scale Recipe Material Characteristics ............................................................... 16

Table 5.2: Final Compost Characteristics of Phase 1 Recipes............................ 17

Table 5.3: Compost Characteristics of Phase 2 Recipes.................................... 20

Table 5.4: Initial Particle Size Analysis of Phase 2 Recipes ............................... 20

\section{LIST OF FIGURES}

Figure 4.1: Phase 1 Environmental Chamber .................................................. 10

Figure 4.2: Phase 1 Reactors Inside Environmental Chamber ......................... 11

Figure 4.1: Phase 2 Compost Reactors ............................................................ 11

Figure 5.1: VS Reduction as a Function of Moisture Content for Phase 1 Recipes. 18

Figure 5.2: Volume Reductions for Phase 2 Recipes ...................................... 22

Figure 5.3: Temperature Measurements for Phase 2 Recipes.............................. 23

Figure 5.4: Volatile Solids Reduction for Phase 2 Recipes ............................... 26

Figure 5.5: Hemicellulose Degradation During Phase 2 Composting.................. 27

Figure 5.6: Cellulose Degradation During Phase 2 Composting.......................... 29

Figure 5.7: Lignin Degradation During Phase 2 Composting ............................ 30 


\section{LIST OF EQUATIONS}

Equation 4-1: Compressive Stress ………………...................................... 12

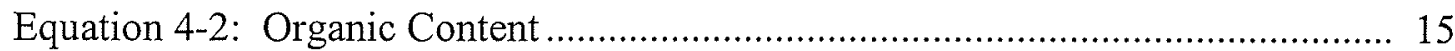




\section{NOMENCLATURE AND ABBREVIATIONS}
$\mathrm{ADF}$
acid detergent fibre
APHA
American Public Health Association
ASTM American Society for Testing and Materials
$\mathrm{C}: \mathrm{N}$
carbon to nitrogen ratio
$\mathrm{C}_{U}$
coefficient of uniformity
d
depth
$\mathrm{D}_{10}$
particle size such that $10 \%$ of particles are smaller than that size
$\mathrm{D}_{30}$
particle size such that $30 \%$ of particles are smaller than that size
$\mathrm{D}_{60}$
particle size such that $60 \%$ of particles are smaller than that size
FAS free air space
FS $\quad$ fixed solids
g gravitational constant
MC moisture content
$\mathrm{N} \quad$ nitrogen
NDF neutral detergent fibre
OC organic carbon
$\mathrm{P}_{\mathrm{d}} \quad$ stress at depth, $\mathrm{d}$
$\rho_{\text {wbd }} \quad$ wet bulk density
TKN total Kjeldahl nitrogen
TS total solids
USCC United States Composting Council
VS volatile solids 


\section{ACKNOWLEDGEMENTS}

Thank you to Dr. Daryl McCartney for both his patience and guidance throughout my research. Special thanks to Ms. Judy Tingley and Mrs. Janice Haines for their laboratory expertise.

This research was funded by the University of Manitoba and the Waste Reduction and Pollution Prevention (WRAPP) fund from Manitoba Conservation. Straw was supplied by Dow BioProducts Ltd. 


\subsection{INTRODUCTION}

Dow BioProducts Ltd. operates a manufacturing facility located near Elie, Manitoba that utilizes straw from the surrounding area to produce strawboard. Each year approximately 6,000 wet tonnes (wt) of straw become too wet for use as feedstock (i.e. $>25 \%$ moisture content). To date there is approximately 60,000 wt of unusable straw stored at the facility in $1 \mathrm{~m} \times 1 \mathrm{~m} \times 2 \mathrm{~m}$ rectangular bales. The manufacturing process also produces, on average, 5,000 wt/year of fine process residuals (unders). Together, the wet straw and unders represent a significant annual waste stream from the strawboard manufacturing facility.

Composting technology has the potential of addressing this waste stream and diverting organic material from a landfill to produce a valuable soil conditioner. However, the lignified fibre composition and high carbon content that makes straw an appropriate structural material for producing strawboard also attributes to the long composting times needed to degrade it. Limited research has been completed in composting a high carbon content material such as straw.

Therefore, to optimize the composting process, the selection of the correct feedstocks and feedstock proportions is critical. Potential compost recipes would include the two components of the plant's waste stream (straw and unders) and require the addition of water to enable an efficient composting process. As a nitrogen amendment, a lagoon mixture obtained from the James Valley Colony, a Hutterite village, was used in select compost recipes to potentially decrease the duration of the active composting period. James Valley Colony lies approximately six kilometres southwest of the strawboard manufacturing facility and uses a lagoon which stores manure from its livestock 
operations. This lagoon mixture is used as fertilizer for the Colony's crops but also has the possibility of becoming a nitrogen source for the composting of straw at the strawboard facility.

The rates and degree of degradation as well as volume reduction are of great importance for the design of a full-scale straw composting facility. However, the effects of particle size on the composting of a high carbon material such as straw have not been well documented and an assessment of how particle size impacts straw composting could result in potential cost savings with regards to straw shredding. 


\subsection{LITERATURE REVIEW}

Straws vary greatly in their chemical composition according to variety and age. The approximate chemical composition of straw is $36 \%$ cellulose, $25 \%$ hemicellulose, and 18\% lignin (Rykens, 1977). The remaining portions are composed of salts, insoluble ash (silica) and various other organic compounds. The rates and degree of straw degradation is therefore largely dependent upon the characteristics of the lignocellulosic structure of straw.

Cellulose is a long chain of glucose molecules. The simplicity of the cellulosic structure, using repeated identical bonds, means that only a small number of enzymes are required to degrade this material. Hemicelluloses are branched polymers of xylose, arabinose, galactose, mannose, and glucose. Hemicelluloses bind bundles of cellulose fibrils to form microfibrils, which enhance the stability of the cell wall. They also cross-link with lignin, creating a complex web of bonds which provide structural strength, but also challenge microbial degradation (Ladisch et al., 1983; Lynch, 1992). Lignin is a complex polymer of phenylpropane units, which are cross-linked to each other with a variety of chemical bonds. This complexity has impeded the understanding of its effects on microbial degradation. However, some organisms, particularly fungi, have developed the necessary enzymes to degrade lignin. Actinomycetes can also decompose lignin, but typically degrade less than 20 percent of the total lignin present (Crawford, 1986; Basaglia et al., 1992).

Because lignin is the most recalcitrant component of the plant cell wall, the higher the proportion of lignin the lower the bioavailability of the substrate. The effect of lignin on the bioavailability of outer cell wall components is thought to be largely a physical restriction with lignin molecules reducing the surface area available to enzymatic 
penetration and activity (Haug, 1993). Thus, the creation of composting conditions which favour the growth of fungi, including adequate moisture and temperature as well as an appropriate particle size to maintain a balance between available surface area for enzymatic activity and available oxygen to maintain suitable aerobic conditions, all appear to be important in encouraging decomposition and are essential for efficient straw composting. In addition, as straw is a high carbon material and slow to degrade, a nitrogen amendment is usually added to the composting process to reduce the carbon to nitrogen ratio and enhance the composting process.

Many researchers have observed the degradation of hemicellulose and cellulose in straw composting (Epstein, 1997; Eklind, 1998). However, the effects of particle size on the composting of a high carbon material such as straw have not been well documented. 


\subsection{OBJECTIVES}

Although the rates and degree of degradation as well as volume reduction are of great importance for the design of a full-scale straw composting facility, an assessment of how particle size impacts straw composting could result in potential cost savings with regards to straw shredding during feedstock preparation. The specific research objectives of this two-phase experiment were to:

- Determine the optimal feedstock proportions for straw composting by adding water, process unders, and nitrogen to the compost recipe .

- Evaluate the effects of particle size on compost degradation and volume reduction.

- Monitor the rate of degradation (measured by VS reduction) and degree of degradation (measured by lignin, cellulose, and hemicellulose) over a typical Canadian Prairie summer composting period.

Phase 1 of the experiment attempted to ascertain optimal moisture levels in a purely straw-based composting recipe. Also, the addition of unders to the straw feedstock would not only utilize a by-product of the strawboard manufacturing process but investigate the potential benefits to reducing the average particle size of the compost matrix and increasing the surface area available for straw degradation. The addition of the lagoon mixture explored the potential benefits of adding a nitrogen source to a highcarbon material such as straw.

Upon completion of Phase 1 of the experiment, it was learned that the historic waste stream at the strawboard manufacturing facility (process unders and straw too wet for use in the manufacturing process) had been eliminated. Process unders were now being incorporated into the strawboard without any effects on the physical properties of the 
strawboard. Additionally, better management practices were initiated in the stack yard such as covering the straw stacks with tarpaulins and constructing raised gravel bases for the straw stacks which eliminated wet straw from the annual waste stream. Therefore, Phase 2 of the experiment focused solely on the wet straw stockpiled at the facility. Using the moisture content results for the straw-based composting recipes from Phase 1, Phase 2 of the experiment further investigated the effects of particle size on the degree and rate of degradation during straw composting. 


\subsection{MATERIALS AND METHODS}

Phase 1 of the research was a preliminary investigation that employed small bench-scale compost reactors containing various recipes using three feedstock materials (straw, process unders, and lagoon mixture) at different moisture levels in an attempt to gain an understanding of composting effectiveness and to aid in the selection of several recipes that could be used for Phase 2 research. The recipe variations used formed the basis for comparing the impact of: (1) water addition; (2) unders addition; and (3) nitrogen addition in the form of a lagoon mixture.

Phase 2 was a more in-depth study focused solely on the straw feedstock utilizing four unshredded/shredded straw combinations (by volume): (1) all unshredded straw, (2) 2/3 unshredded straw, 1/3 shredded straw, (3) 1/3 unshredded straw, 2/3 shredded straw, and (4) all shredded straw. Process unders were not included in this phase of the experiment since process improvements at the strawboard manufacturing plant led to the elimination of the unders from the waste stream. Specific objectives for Phase 2 were to: (1) investigate the effects of particle size on compost degradation and volume reduction; and (2) monitor the rate of degradation (measured by VS reduction) and degree of degradation (measured by lignin, cellulose, and hemicellulose) over a typical Canadian Prairie summer composting period to estimate the ultimate degradability of straw compost.

\subsection{Materials}

The straw used in Phases 1 and 2 was obtained from the Dow BioProducts stack yard and is mainly AC Barrie wheat straw, a predominant variety of wheat farmed in the $50-80 \mathrm{~km}$ feedstock collection radius of the strawboard manufacturing facility. The stack yard was 
estimated as $75 \% \mathrm{AC}$ Barrie wheat straw with the remainder composed of up to 15 other wheat straw varieties. The straw bales in the stack yard all had common dimensions of $1 \mathrm{~m} \times 1 \mathrm{~m} \times 2 \mathrm{~m}$. Straw used for Phase 1 was sampled from 1998 stock on June 18, 2001. Five separate straw bales from the 1998 stock were sampled and three straw subsamples (4-8 L each) were collected from each straw bale based upon a randomly selected position within each bale. The three straw sub-samples collected from each bale were then combined to form a composite sample. The straw was shredded using a 3 horse power Chipper/Shredder, (Crary Bearcat Model 530, West Fargo, North Dakota, USA) with a screen size of approximately $10 \mathrm{~cm}$. Straw used for Phase 2 was sampled from 1997, 1998, 1999, and 2000 stock on April 10, 2002 from several locations in the stack yard. A total of eight straw bales (two from each year) were used to obtain representative straw samples following the method outlined for Phase 1 of the experiment with the exception that the sub-samples were approximately 30-50 L each. Portions of the Phase 2 straw samples collected from the stack yard were shredded using a 460 horsepower tub grinder (Haybuster Model H1100E, Jamestown, ND, 58401, USA) that is owned and operated by Dow BioProducts.

The process unders used for Phase 1 were obtained from the Dow BioProducts strawboard manufacturing facility. The unders are removed from the strawboard manufacturing process at the wet screens $(0.3 \times 0.2 \mathrm{~mm}$ slots $)$ just before the dryers.

The lagoon mixture was obtained from James Valley Colony, a Hutterite farming colony. Colony farming operations include livestock such as diary cows (approximately 55 head), laying hens (approximately 14,000 head), and farrow-to-finish hogs (approximately 600 head). Manure from the chicken and hog barns flows by gravity to a collection pit and is 
then pumped to a two-cell lagoon from which $19,000 \mathrm{~m}^{3}$ of the material is used annually as fertilizer on the Colony's fields.

\subsection{Composting Set-Up}

The bench-scale composting period for Phase 1 was nine weeks in duration. Seven different recipes were investigated in duplicate resulting in a total of 14 reactors. The recipe descriptions are shown in Table 4.1. Recipes $2 \mathrm{~A}$ to $2 \mathrm{E}$ were based on the projected long-term waste generation at the strawboard manufacturing facility in 2001 (6,000 wet tonnes straw/year, 5,000 wet tonnes process unders/year). The various recipes were selected to answer three specific research questions for Phase 1. The questions and recipes are summarized in Table 4.2 .

Table 4.1: Phase 1 Small Bench-scale Reactor Recipe Descriptions.

\begin{tabular}{|c|c|c|c|c|c|}
\hline \multirow[b]{2}{*}{ Reactor Recipe } & \multicolumn{4}{|c|}{ Feedstock (\% of wet weight) } & \multirow[b]{2}{*}{$\begin{array}{c}\text { Initial MC } \\
(\%) \\
\end{array}$} \\
\hline & Straw & Unders & $\begin{array}{l}\text { Lagoon } \\
\text { Mixture }\end{array}$ & Water & \\
\hline $1 \mathrm{~A}$ & 100 & NA & NA & NA & 57 \\
\hline $1 \mathrm{~B}$ & 70 & NA & $\mathrm{NA}$ & 30 & 70 \\
\hline $2 \mathrm{~A}$ & 55 & 45 & NA & NA & 40 \\
\hline $2 \mathrm{~B}$ & 41 & 34 & $\mathrm{NA}$ & 25 & 55 \\
\hline $2 \mathrm{C}$ & 34 & 28 & $\mathrm{NA}$ & 38 & 62.5 \\
\hline $2 \mathrm{D}$ & 27 & 22 & $\mathrm{NA}$ & 51 & 70 \\
\hline $2 \mathrm{E}$ & 32 & 25 & 43 & $\mathrm{NA}$ & 62.5 \\
\hline
\end{tabular}

Note: $\mathrm{NA}=$ not added

Table 4.2: Phase 1 Recipes Used for Comparisons

\begin{tabular}{|c|c|}
\hline Research Questions & Recipes Used for Comparison \\
\hline Impact of water addition & $1 \mathrm{~A}$ and $1 \mathrm{~B} ; 2 \mathrm{~A}$ to $2 \mathrm{D}$ \\
\hline Impact of under addition & $1 \mathrm{~A}$ and $2 \mathrm{~B} ; 1 \mathrm{~B}$ and $2 \mathrm{D}$ \\
\hline Impact of additional N source & $2 \mathrm{C}$ and $2 \mathrm{E}$ \\
\hline
\end{tabular}


Plastic 3.8 litre pails were used as bench-scale compost reactors and placed in an environmental chamber $(1.2 \mathrm{~m} \times 1.0 \mathrm{~m} \times 1.2 \mathrm{~m})$ maintained at $55^{\circ} \mathrm{C}$ (Larsen and McCartney, 2000) as shown in Figure 4.1. The compost reactors were laid on the floor of the environmental chamber with the reactor lids fitted loosely on top to minimize moisture loss while still maintaining aerobic conditions. Open containers of water were also placed on the floor of the chamber to increase chamber humidity and minimize reactor moisture losses as shown in Figure 4.2. Reactors were mixed and readjusted to target moisture contents on 3-4 day intervals. Initial amounts of dry material in each reactor ranged from $80-150$ grams.

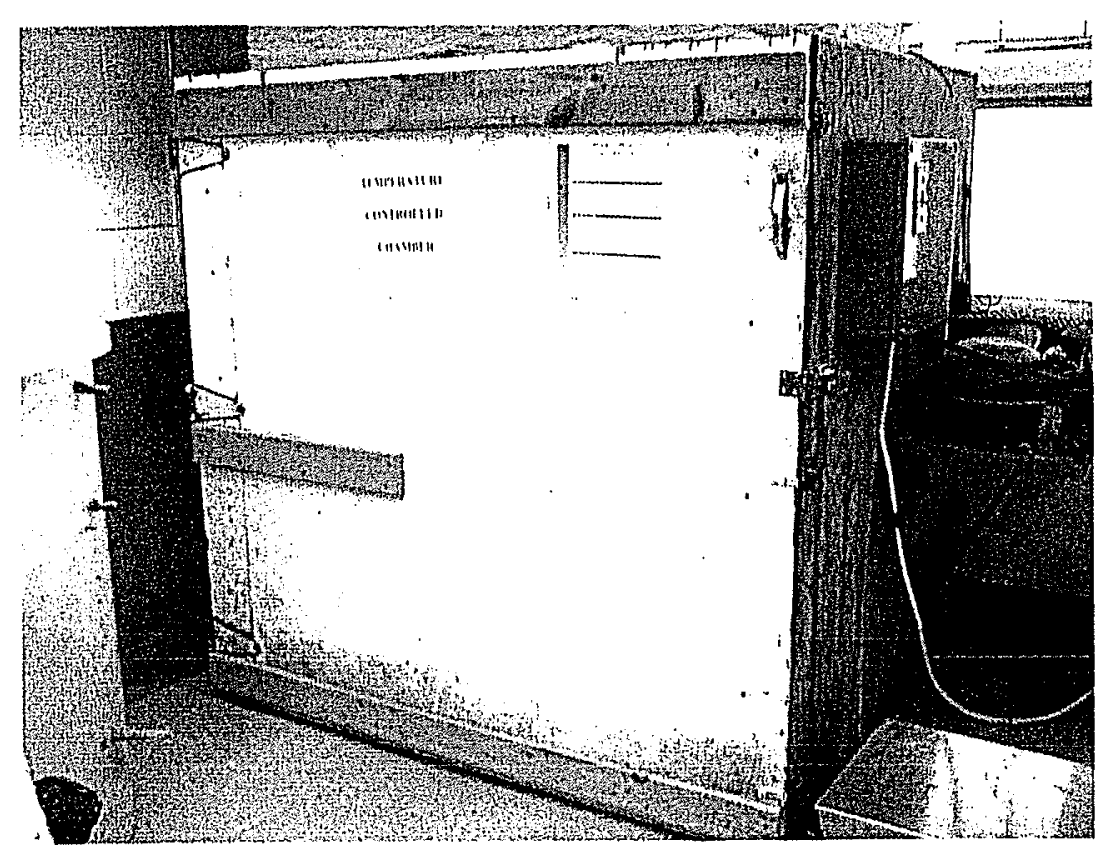

Figure 4.1: Phase 1 environmental chamber. 


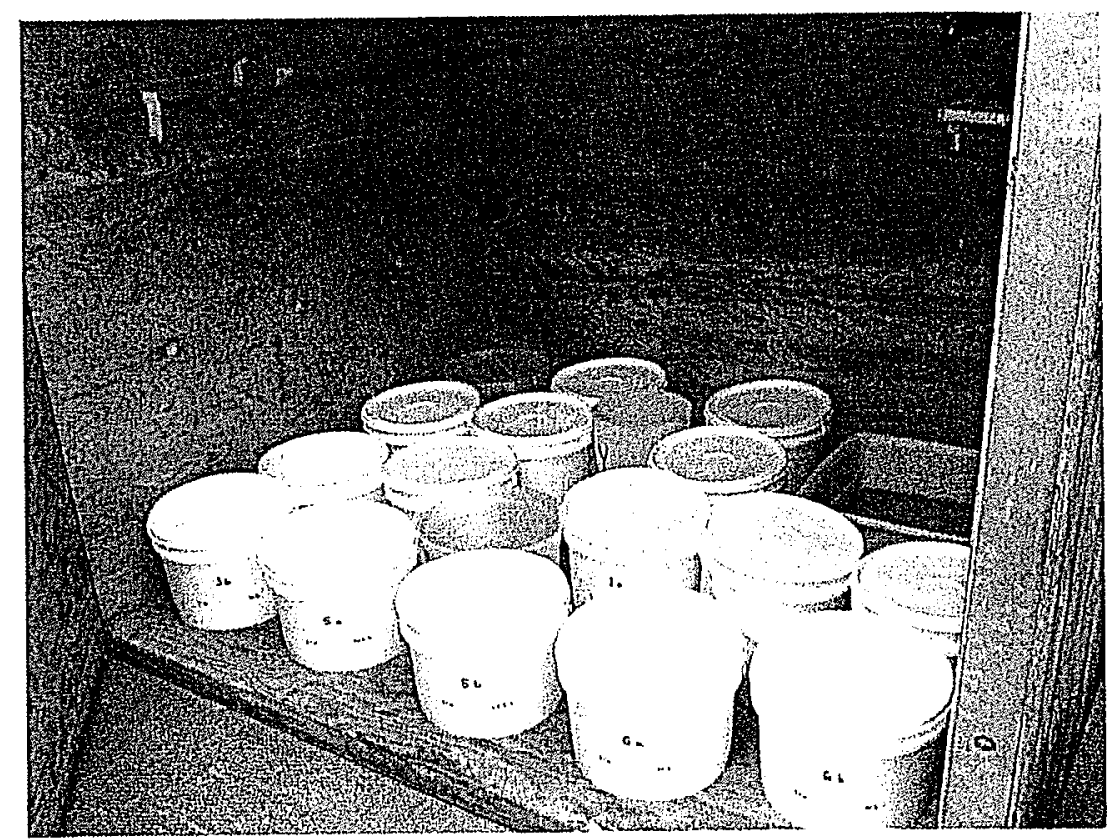

Figure 4.2: Phase 1 reactors inside environmental chamber.

Phase 2 of the experiment was a more in-depth study lasting a typical six-month composting season in the Canadian Prairies. Four recipes of varying straw particle sizes were composted in duplicate $140 \mathrm{~L}$ plastic containers as shown in Figure 4.3 (Schaefer System International Limited Compostainer Model, Brampton, Ontario, Canada).
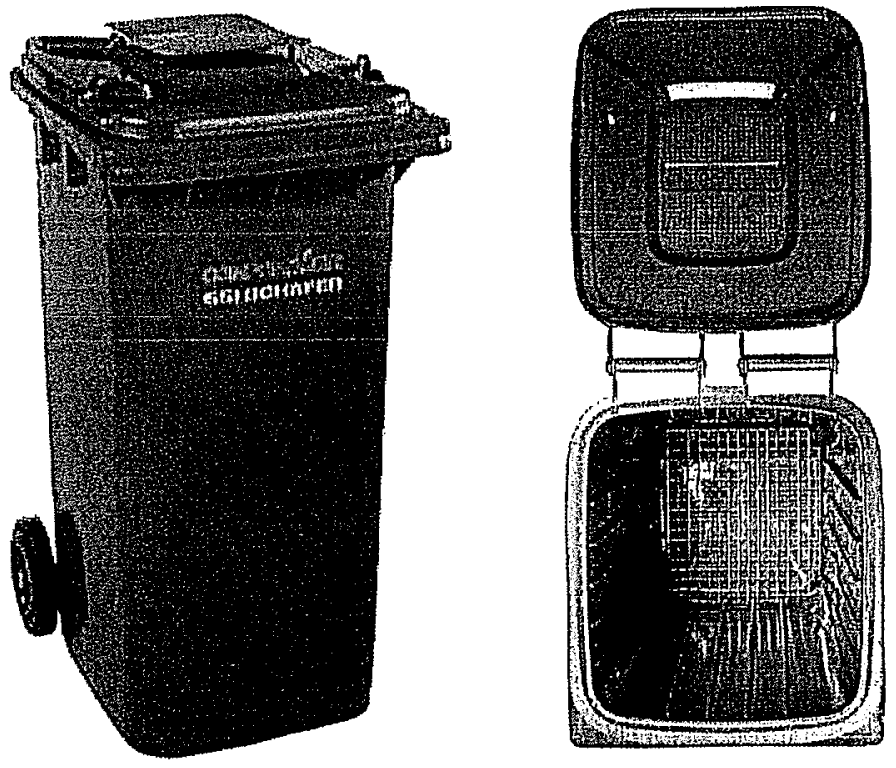

Figure 4.3: Phase 2 compost reactors. 
Three oxygen/temperature sampling ports $(1 \mathrm{~cm}$ diameter) located 10,35 , and $50 \mathrm{~cm}$ above the container bottoms were drilled into two sides of each container. The containers were placed in an environmental chamber where the average temperature and relative humidity for the duration of the experiment was $42.6^{\circ} \mathrm{C}$ and $41.7 \%$ respectively. Passive aeration was used as opposed to forced aeration. The contents of each reactor were mixed weekly to allow for water addition as needed to maintain a moisture content of $70 \%$ (wet basis) and to reestablish the structure and pore space of the compost matrix to allow for adequate distribution of air. Loads were placed on top of the reactor contents to simulate a $2.5 \mathrm{~m}$ depth within a windrow compost pile. The target moisture content of 70 $\%$ was chosen based on the results of Phase 1 of the experiment and to ensure that with the simulated depth of $2.5 \mathrm{~m}$, a free air space (FAS) greater than $30 \%$ for all recipes at could be maintained. FAS refers to the inter-particle voids in the compost matrix and is discussed in Eftoda and McCartney (2002). The FAS level of 30\% is recommended by Rynk (1992). The loads needed were calculated by first running a series of tests on each recipe to determine the wet bulk density in a large cylindrical container, or biocell (Wizbicky, 2002), with added loads of 3, 6, and $10 \mathrm{kPa}$. An equation from McCartney and Chen (2001):

$$
P_{d}=\rho_{w b d} d g \quad \text { (McCartney and Chen, 2001) }
$$

where $P_{d}$ is the stress at any depth $(\mathrm{kPa}) ; \rho_{\text {wbd }}$ is the material wet bulk density $\left(\mathrm{kg} \mathrm{m}^{-3}\right)$; is the material depth $(\mathrm{m})$; and $\mathrm{g}$ is gravity $\left(9.81 \mathrm{~m} / \mathrm{s}^{2}\right)$, was used to calculate depth so a plot of compost pile depth versus FAS could be constructed (included in Appendix A). The loads used for Phase 2 of the experiment (Day 0 - Day 119 only) were 2.70, 3.54, 5.41 , and $6.88 \mathrm{kPa}$ for Recipes 1 through 4, respectively which, using the average cross- 
sectional area of the compost reactors, translated into weights of $38.70,50.75,77.55$, and $98.62 \mathrm{~kg}$, respectively. A decision was made to remove the loads on Day 119 since they were no longer compressing the compost material (volume and particle size reduction were considerable at this point). The initial masses of unshredded and shredded straw used in each recipe were calculated on a volume basis and are shown in Table 4.3. The total masses of straw in Recipes 1 through 4 were $12.3,19.8,27.3$, and $34.8 \mathrm{~kg}$, respectively.

Table 4.3: Masses of unshredded and shredded straw used in Phase 2 recipes.

\begin{tabular}{|c|c|c|}
\hline \multirow{2}{*}{ Recipe } & \multicolumn{2}{|c|}{ Mass of Straw (kg) } \\
\cline { 2 - 3 } & Shredded & Unshredded \\
\hline $\begin{array}{c}1 \\
\text { (unshredded straw) }\end{array}$ & 12.3 & 0 \\
\hline $\begin{array}{c}2 \\
(2 / 3 \text { unshredded straw, } \\
1 / 3 \text { shredded straw) }\end{array}$ & 8.2 & 11.6 \\
\hline $\begin{array}{c}3 \\
(1 / 3 \text { unshredded straw, } \\
2 / 3 \text { shredded straw) }\end{array}$ & 4.1 & 23.2 \\
\hline $\begin{array}{c}4 \\
\text { (shredded straw) }\end{array}$ & 0 & 34.8 \\
\hline
\end{tabular}

\subsection{Sampling}

Initial samples were taken for both phases of the experiment. Phase 1 sampling continued every 3-4 days thereafter - the weight of the reactor contents being recorded following each moisture readjustment to track weight loss. Phase 2 employed a weekly sampling protocol in which the contents of each reactor were spread out on a tarp and ten approximately equal random samples of $50 \mathrm{~g}$ each were taken to form a composite sample. Quartering techniques were then used to obtain a $50 \mathrm{~g}$ sample for analysis. The cumulative amounts of sample removed from each reactor throughout the entire 
composting period range from 4.5 to $9.1 \%$ of the initial dry weight. Temperature and oxygen measurements were taken at a horizontally centered position in the composting reactor using the three oxygen/temperature sampling ports. Reported values are an average of measurements taken from the three sampling ports for each reactor. Temperature measurements were taken daily from day 2 to day 56 only, at which time the temperature profile for each compost reactor had reached a plateau. Pore space oxygen measurements were made during a one-week period from day 13 to day 20 .

\subsection{Physical and Chemical Analyses}

In Phase 1 of the experiment, moisture content was monitored every 3 to 4 days using an Infra-red Moisture Balance (CSC Scientific Company, Inc., Fairfax, Virginia) with a 125watt infrared lamp (McCartney and Tingley, 1998). The 5-gram samples used for moisture determination were returned to the reactor.

The initial compost feedstock materials (straw and manure) and the initial and finished compost recipes were analysed for total solids (TS), volatile solids (VS), fixed solids (FS), organic carbon (OC), total Kjeldahl nitrogen (TKN), and fibre composition (lignin, cellulose, and hemicellulose). Phase 2 included analyses of bulk density, particle density, FAS, and particle size in addition to weekly analysis of TS, VS, FS, OC, and fibre composition. TS, VS, and FS were determined using standard methods $2540 \mathrm{~B}$ and $\mathrm{E}$ (APHA, 1995). The OC was calculated using Equation 2 where OC and FS are based on the dry weight fraction. TKN analysis was conducted by following the "Micro-Kjeldahl Digestion Followed by Steam Distillation: Without Pretreatment to Include $\mathrm{NO}_{2}^{-}$and $\mathrm{NO}_{3}{ }^{-}$Quantitatively" method (Carter, 1993). The $\mathrm{C}: \mathrm{N}$ ratio was calculated using the $\mathrm{OC}$ and TKN results. For Phase 1, mass balances were conducted to determine VS reduction 
and $\mathrm{N}$ loss. Phase 2 fibre analysis was based on separate determinations of neutral detergent fibres (NDF), acid detergent fibres (ADF) and lignin. ADF (Komarek et al. 1993) and NDF (Komarek et al. 1994) were analysed using the ANKOM Fibre Analyser \#F200 (Fairport, NY). The NDF fraction, that material which is not solubilised by neutral detergent, is an estimate of total fibre content (lignin, cellulose, and hemicellulose), while the ADF fraction, that material which is not solubilised by acid detergent, contains cellulose and lignin. Lignin was determined on the remaining material after $\mathrm{ADF}$ analysis and further treatment with $72 \%$ sulphuric acid (van Soest, 1963). Results from NDF, ADF and lignin determinations were used to estimate contents of hemicellulose (NDF - ADF) and cellulose (ADF - lignin). Bulk density was determined using the procedures in method 07.01-A proposed by USCC (1997), with the exception that the vessel used had a diameter of $25 \mathrm{~cm}$ and a height of $57 \mathrm{~cm}$. Particle density was determined using the water pycnometer method (Klute et al. 1986) with the exception that a 1 litre volumetric flask was substituted for the pyconometer in order to accommodate larger sample volumes. Details on FAS calculations can be found in Eftoda and McCartney (2002). Particle size analysis was completed using the ASTM standard method D 422-63 (1990). Hand sieving was used with sieve screen sizes of $37.5,19.0,9.5,4.75,2.36,1.18,0.6,0.3$, and $0.15 \mathrm{~mm}(1.5,0.75,0.375,0.187,0.091$, $0.0465,0.0236,0.0118,0.00591$ inches). Temperature and oxygen measurements were made using an oxygen-temperature probe (Demista Instruments Model No. OT-21, Mt. Prospect, Illinois, 60056, USA).

$$
O C=\frac{(1-F S)}{1.8} \quad(\text { Haug 1993, Liao et al. 1995) }
$$




\subsection{RESULTS AND DISCUSSION}

A summary of the physical and chemical characteristics for the raw feedstock materials and initial compost recipes for Phases 1 and 2 is presented in Table 5.1.

\subsection{Phase 1 Composting}

The final material characteristics of the compost recipes were analyzed to determine the composting properties of each recipe over the entire nine-week composting period. Table 5.2 compares the final $\mathrm{C}: \mathrm{N}$ ratios as well as the mass balance results for VS reduction and $\mathrm{N}$ loss in each compost recipe.

Table 5.1: Phase 1 \& 2 Feedstock and Initial Bench-scale Recipe Material Characteristics

\begin{tabular}{|c|c|c|c|c|c|c|c|c|}
\hline \multicolumn{2}{|c|}{ Materials } & \multirow{2}{*}{$\begin{array}{c}\begin{array}{c}\% \text { MC } \\
(\mathrm{db})\end{array} \\
57 \\
(16.6)\end{array}$} & \multirow{2}{*}{$\begin{array}{c}\begin{array}{c}\text { \%TKN } \\
(\mathrm{db})\end{array} \\
1.43 \\
(0.22)\end{array}$} & \multirow{2}{*}{$\begin{array}{c}\begin{array}{c}\% \mathbf{O C} \\
(\mathrm{db})\end{array} \\
45.3 \\
(2.2)\end{array}$} & \multirow{2}{*}{$\begin{array}{c}C: N \\
\text { (wt:wt) } \\
28.5\end{array}$} & \multirow{2}{*}{$\begin{array}{c}\text { \% Lignin } \\
\text { n.d. }\end{array}$} & \multirow{2}{*}{$\begin{array}{c}\begin{array}{c}\% \text { Cellulose } \\
(\mathrm{db})\end{array} \\
\text { n.d. }\end{array}$} & \multirow{2}{*}{$\frac{\begin{array}{c}\% \text { Hemicellulose } \\
\text { (db) }\end{array}}{\text { n.d. }}$} \\
\hline 7 & Straw & & & & & & & \\
\hline : & Unders & 18 & 1.48 & 45.6 & 31.0 & n.d. & n.d. & n.d. \\
\hline E & $\begin{array}{l}\text { Lagoon } \\
\text { Mixture }\end{array}$ & $\begin{array}{c}93 \\
(0.2) \\
\end{array}$ & $\begin{array}{c}23.5 \\
(13.8)\end{array}$ & $\begin{array}{l}13.9 \\
(1.5) \\
\end{array}$ & 0.6 & n.d. & n.d. & n.d. \\
\hline \multirow{7}{*}{ 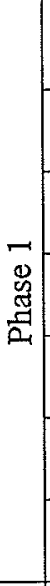 } & Recipe $1 \mathrm{~A}$ & 57 & $\begin{array}{c}1.43 \\
(0.22) \\
\end{array}$ & $\begin{array}{l}45.7 \\
(0.1) \\
\end{array}$ & 32.0 & n.d. & n.d. & n.d. \\
\hline & Recipe 1B & 70 & $\begin{array}{c}1.43 \\
(0.22) \\
\end{array}$ & $\begin{array}{l}44.3 \\
(0.6) \\
\end{array}$ & 31.0 & n.d. & n.d. & n.d. \\
\hline & Recipe 2A & 40 & $\begin{array}{c}1.29 \\
(0.13) \\
\end{array}$ & $\begin{array}{l}44.8 \\
(0.2) \\
\end{array}$ & 34.7 & n.d. & n.d. & n.d. \\
\hline & Recipe 2B & 55 & $\begin{array}{c}1.29 \\
(0.13)\end{array}$ & $\begin{array}{l}44.5 \\
(0.1) \\
\end{array}$ & 34.5 & n.d. & n.d. & n.d. \\
\hline & Recipe 2C & 62.5 & $\begin{array}{c}1.29 \\
(0.13) \\
\end{array}$ & $\begin{array}{l}44.8 \\
(0.3) \\
\end{array}$ & 34.7 & n.d. & n.d. & n.d. \\
\hline & Recipe 2D & 70 & $\begin{array}{c}1.29 \\
(0.13)\end{array}$ & $\begin{array}{l}45.1 \\
(0.4) \\
\end{array}$ & 35.0 & n.d. & n.d. & n.d. \\
\hline & Recipe 2E & 62.5 & $\begin{array}{c}1.55 \\
(0.18) \\
\end{array}$ & $\begin{array}{l}44.6 \\
(0.0) \\
\end{array}$ & 28.8 & n.d. & n.d. & n.d. \\
\hline 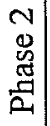 & $\begin{array}{l}\text { Recipe } \\
1,2,3,4\end{array}$ & 70 & $\begin{array}{c}0.76 \\
(0.06)\end{array}$ & $\begin{array}{l}48.9 \\
(0.8)\end{array}$ & 64.2 & 11.4 & 42.5 & 19.6 \\
\hline
\end{tabular}

Notes:

1) Values shown are mean values (standard deviations shown in parentheses).

2) n.d. = not determined 
Table 5.2: Final Compost Characteristics of Phase 1 Recipes

\begin{tabular}{|c|c|c|c|c|}
\hline Recipe & $\begin{array}{c}\% \text { VS } \\
\text { Reduction } \\
\end{array}$ & $\% \mathrm{~N}$ Loss & $\begin{array}{c}\% \text { Weight Reduction } \\
\text { (dry basis) }\end{array}$ & $\begin{array}{c}\text { Final C:N } \\
\quad \text { Ratio } \\
\end{array}$ \\
\hline $1 \mathrm{~A}$ & $\begin{array}{l}45.8 \\
(0.0) \\
\end{array}$ & $\begin{array}{l}17.0 \\
(5.5) \\
\end{array}$ & $\begin{array}{r}39.3 \\
(0.1) \\
\end{array}$ & 20.9 \\
\hline 1B & $\begin{array}{l}58.2 \\
(2.0)\end{array}$ & $\begin{array}{c}8.5 \\
(3.2) \\
\end{array}$ & $\begin{array}{l}48.9 \\
(2.0)\end{array}$ & 14.2 \\
\hline $2 \mathrm{~A}$ & $\begin{array}{l}46.9 \\
(2.1)\end{array}$ & $\begin{array}{l}17.1 \\
(3.1)\end{array}$ & $\begin{array}{l}38.9 \\
(2.8)\end{array}$ & 22.2 \\
\hline $2 \mathrm{~B}$ & $\begin{array}{l}58.7 \\
(0.2) \\
\end{array}$ & $\begin{array}{l}18.8 \\
(0.4)\end{array}$ & $\begin{array}{l}48.7 \\
(0.1) \\
\end{array}$ & 17.6 \\
\hline $2 \mathrm{C}$ & $\begin{array}{l}65.9 \\
(2.7)\end{array}$ & $\begin{array}{l}23.4 \\
(1.2)\end{array}$ & $\begin{array}{l}55.4 \\
(2.0)\end{array}$ & 15.5 \\
\hline $2 \mathrm{D}$ & $\begin{array}{l}71.3 \\
(1.5) \\
\end{array}$ & $\begin{array}{l}18.6 \\
(4.7)\end{array}$ & $\begin{array}{l}58.8 \\
(1.6) \\
\end{array}$ & 12.3 \\
\hline $2 \mathrm{E}$ & $\begin{array}{l}69.9 \\
(1.5)\end{array}$ & $\begin{array}{l}32.4 \\
(0.1) \\
\end{array}$ & $\begin{array}{l}57.9 \\
(1.0)\end{array}$ & 12.8 \\
\hline
\end{tabular}

Note: Characteristics shown here are averages of duplicate reactors for each recipe (standard deviations shown in parentheses).

For the recipes consisting of only straw ( $1 \mathrm{~A} \& 1 \mathrm{~B})$, it was found that an increase in moisture content resulted in greater VS reduction. A similar trend was seen for the recipes including straw and unders - increased VS reduction with increased MC where VS reduction reached a maximum of $71.3 \%$ for Recipe 2D. Figure 5.1 more clearly indicates the strong positive linear trend $\left(\mathrm{R}^{2}=0.9983\right)$ associated with $\mathrm{MC}$ for Recipes $2 \mathrm{~A}, 2 \mathrm{~B}, 2 \mathrm{C}$, and 2D. Using Figure 5.1, comparisons between Recipes $1 \mathrm{~A} \& 2 \mathrm{~B}$ and Recipes 1B \& 2D showed an increased VS reduction with the addition of unders to the compost mixture. The increased VS reduction, an indication of increased degradability, was likely due to better degradability of the unders material. Since decomposition occurs on particle surfaces, the smaller particle size of the unders increases the surface area available and therefore improves degradability.

It was also possible to assess the potential advantages of adding a nitrogen source in the form of a lagoon mixture by analyzing VS reduction. Recipes $2 \mathrm{C}$ and $2 \mathrm{E}$ were compared to make this assessment and their initial $\mathrm{N}$ contents and $\mathrm{C}: \mathrm{N}$ ratios are shown in Table 
5.1. Figure 5.1 indicates that the added $\mathrm{N}$ source in Recipe $2 \mathrm{E}$ did not result in a statistically significant advantage over Recipe $2 \mathrm{C}$ with respect to VS reduction. The starting recipe characteristics shown in Table 5.1 indicate only a marginal difference in initial $C: N$ ratio, which may have reduced the expected advantages of the nitrogen addition.

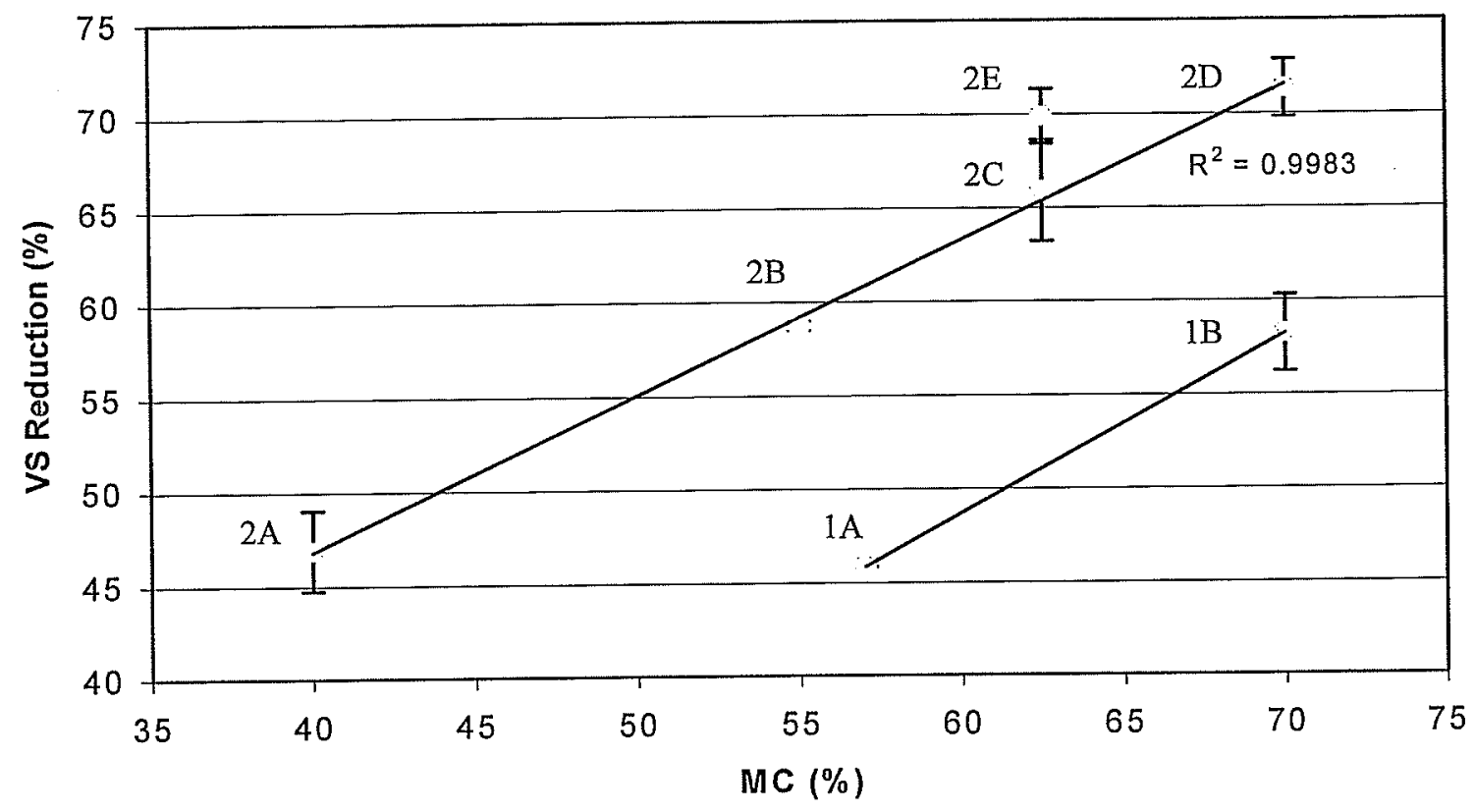

Figure 5.1: VS reduction as a function of moisture content for Phase 1 recipes. Note: Results indicate averages of duplicate reactors. Error bars represent \pm one standard deviation of recipe duplicates.

Assuming the dry weight loss in the reactors was due to VS losses, the VS removal profiles for the entire small bench-scale test period were calculated. For Recipes IA and $1 \mathrm{~B}$, similar drops in \% original VS occurred in the first 20 days but Recipe $1 \mathrm{~B}$, with an increased $\mathrm{MC}$, showed a greater overall reduction for the remainder of the experiment. This trend was similar for Recipes $2 \mathrm{~A}$ to $2 \mathrm{D}$. Similar reductions of VS were exhibited for the first 10 to 12 days of the trial but after this point, differentiation was noticed for the remainder of the experiment with greater reductions of VS occurring for reactors with 
higher moisture contents. Comparisons of the VS removal profiles for Recipes 1A \& 2B and Recipes 1B \& 2D showed that the addition of unders to the compost recipe resulted in increased reduction of original VS by day 10 , once again indicating the greater degradability of the unders when they are added to the compost mixture. By comparing Recipes $2 \mathrm{C} \& 2 \mathrm{E}$ it was noticed that Recipe 2E displayed a quicker initial reduction of VS but after 15 days the decrease in VS for the two treatment recipes was similar. As with the results shown in Figure 5.1, only a small advantage was noticed with the addition of nitrogen to the compost mixture.

The $\mathrm{N}$ loss for each of the compost recipes is shown in Table 5.2. Comparison of Recipes 1A \& 1B (varying only in MC) indicated a smaller $\mathrm{N}$ loss for the recipe with a higher MC (Recipe 1B). This comparison seems to agree well with conventional theory - the higher moisture content in Recipe 1B would be expected to aid in preventing the volatilization of nitrogen in the form of ammonia. No statistical significance was noted between the straw-only recipes and the straw/unders recipes. Additionally, there appears to be no trend with respect to $\mathrm{N}$ loss as $\mathrm{MC}$ increases in straw/unders recipes (Recipes 2A through 2D). The $\mathrm{N}$ loss exhibited for Recipe 2E (containing an additional nitrogen source) was greater than that for Recipe $2 \mathrm{C}$. The greater $\mathrm{N}$ loss was expected since the initial \% TKN was greater for Recipe 2E (see Table 5.1).

\subsection{Phase 2 Composting}

\subsubsection{Particle Size, Mass, Volume and C:N Ratio}

The final material characteristics of the compost recipes used in Phase 2 of the experiment are shown in Table 5.3. Results of the initial particle size analysis are shown in Table 5.4. The particle size such that $10 \%$ of the particles are smaller than that size is 
denoted by $D_{10} . D_{30}$ and $D_{60}$ are defined similarly. The coefficient of uniformity $\left(C_{U}\right)$ indicates the range of particle sizes in the material (i.e. the higher the value of $\mathrm{C}_{U}$ the larger the range of particle sizes).

Table 5.3: Compost Characteristics of Phase 2 Recipes

\begin{tabular}{|c|c|c|c|c|c|c|}
\hline \multirow[b]{2}{*}{ Recipe } & \multicolumn{2}{|c|}{$\begin{array}{c}\text { Dry Bulk Density } \\
\left(\mathrm{kg} / \mathrm{m}^{\mathbf{3}}\right)\end{array}$} & \multirow{2}{*}{$\begin{array}{c}\text { Initial } \\
\text { FAS } \\
(\%)\end{array}$} & \multirow{2}{*}{$\begin{array}{l}\text { \% Weight } \\
\text { Reduction } \\
\text { (dry basis) }\end{array}$} & \multicolumn{2}{|c|}{ C:N Ratio } \\
\hline & Initial & Final & & & Initial & Final \\
\hline $\begin{array}{c}1 \\
\text { (unshredded straw) }\end{array}$ & 20.1 & $\begin{array}{c}219.5 \\
(3.2)\end{array}$ & 87 & $\begin{array}{l}77.5 \\
(1.9) \\
\end{array}$ & 64.2 & 13.5 \\
\hline $\begin{array}{c}2 \\
\text { (2/3 unshredded straw, } \\
1 / 3 \text { shredded straw) }\end{array}$ & 31.4 & $\begin{array}{l}327.0 \\
(37.2)\end{array}$ & 79 & $\begin{array}{l}73.6 \\
(0.9)\end{array}$ & 64.2 & 11.1 \\
\hline $\begin{array}{c}3 \\
\text { (1/3 unshredded straw, } \\
\text { 2/3 shredded straw) }\end{array}$ & 49.1 & $\begin{array}{l}399.5 \\
(43.4)\end{array}$ & 68 & $\begin{array}{l}74.3 \\
(0.2)\end{array}$ & 64.2 & 9.4 \\
\hline $\begin{array}{c}4 \\
\text { (shredded straw) }\end{array}$ & 58.0 & $\begin{array}{l}225.7 \\
(22.6)\end{array}$ & 62 & $\begin{array}{l}67.3 \\
(0.3)\end{array}$ & 64.2 & 10.7 \\
\hline
\end{tabular}

Note: Characteristics shown here are averages of duplicate reactors for each recipe (standard deviations shown in parentheses).

Table 5.4: Initial Particle Size Analysis of Phase 2 Recipes

\begin{tabular}{|c|c|c|c|c|}
\hline Recipe & $\begin{array}{c}\mathbf{D}_{10} \\
(\mathrm{~mm})\end{array}$ & $\begin{array}{c}\mathbf{D}_{\mathbf{3 0}} \\
(\mathrm{mm})\end{array}$ & $\begin{array}{c}\mathbf{D}_{60} \\
(\mathrm{~mm})\end{array}$ & $\begin{array}{c}\mathbf{C}_{\mathbf{U}} \\
\left(\mathrm{D}_{60} / \mathrm{D}_{30}\right)\end{array}$ \\
\hline $\begin{array}{c}1 \\
\text { (unshredded) }\end{array}$ & 1.5 & 3.6 & 8.0 & 5.3 \\
\hline $\begin{array}{c}2 \\
\text { (2/3 unshredded, 1/3 shredded) }\end{array}$ & 1.1 & 2.0 & 4.7 & 4.3 \\
\hline $\begin{array}{c}3 \\
\text { (1/3 unshredded, 2/3 shredded) }\end{array}$ & 1.1 & 2.3 & 4.1 & 3.7 \\
\hline $\begin{array}{c}4 \\
\text { (shredded) }\end{array}$ & 0.9 & 1.8 & 3.2 & 3.6 \\
\hline
\end{tabular}

The initial dry bulk density increased for Recipes 1 through 4 . This was expected due to the decrease in particle size for each recipe. Initial FAS determinations are in agreement with the dry bulk density and particle size analysis. Recipe 1 had the highest initial FAS (87\%) while Recipe 4 had the lowest initial FAS (62\%). An analysis of the initial and final dry bulk densities for each recipe indicated that the recipe with the largest initial 
particle size (Recipe 1) attained the largest increase in dry bulk density (10.9 times the initial) followed by Recipes 2 through 4 at 10.4, 8.1, and 3.9 times their initial dry bulk density. Weight reduction also followed this trend with the exception that Recipe 3 showed a greater weight reduction than Recipe 2. High initial $C: N$ ratios of 64.2 were reduced the most by Recipe 3 (final $\mathrm{C}: \mathrm{N}$ of 9.4). The final $\mathrm{C}: \mathrm{N}$ ratio was highest for Recipe 1 (13.5) after the 180-day composting period.

The volume reductions for each recipe are shown in Figure 5.2. It should be noted that the results shown are an average of duplicate reactors for each recipe and do not take into account the amounts of material withdrawn for weekly sampling purposes. Also, the loads used to simulate a $2.5 \mathrm{~m}$ depth in a windrow compost pile were removed on day 119 of the experiment resulting in a slight "refluffing" of the compost material represented by a mild peak in Figure 5.2. Volume reductions of 50\% were observed by day 50 of the experiment for all recipes. Recipes 2 and 3 showed similar rates of volume reduction before stabilizing at approximately $10 \%$ of their initial volumes indicating that the particles sizes (mixture of unshredded and shredded straw) were more conducive to rapid volume reduction. Recipe 1 did not exhibit the same rates of volume reduction as Recipes 2 and 3 but finished at approximately $10 \%$ of its initial volume as well. Recipe 4 showed the least amount of volume reduction (81\%) perhaps owing to the smaller initial particle size. The additional $10 \%$ of volume reduction attained by Recipes 1,2 , and 3 would have important implications in the design of a full-scale straw composting operation. 


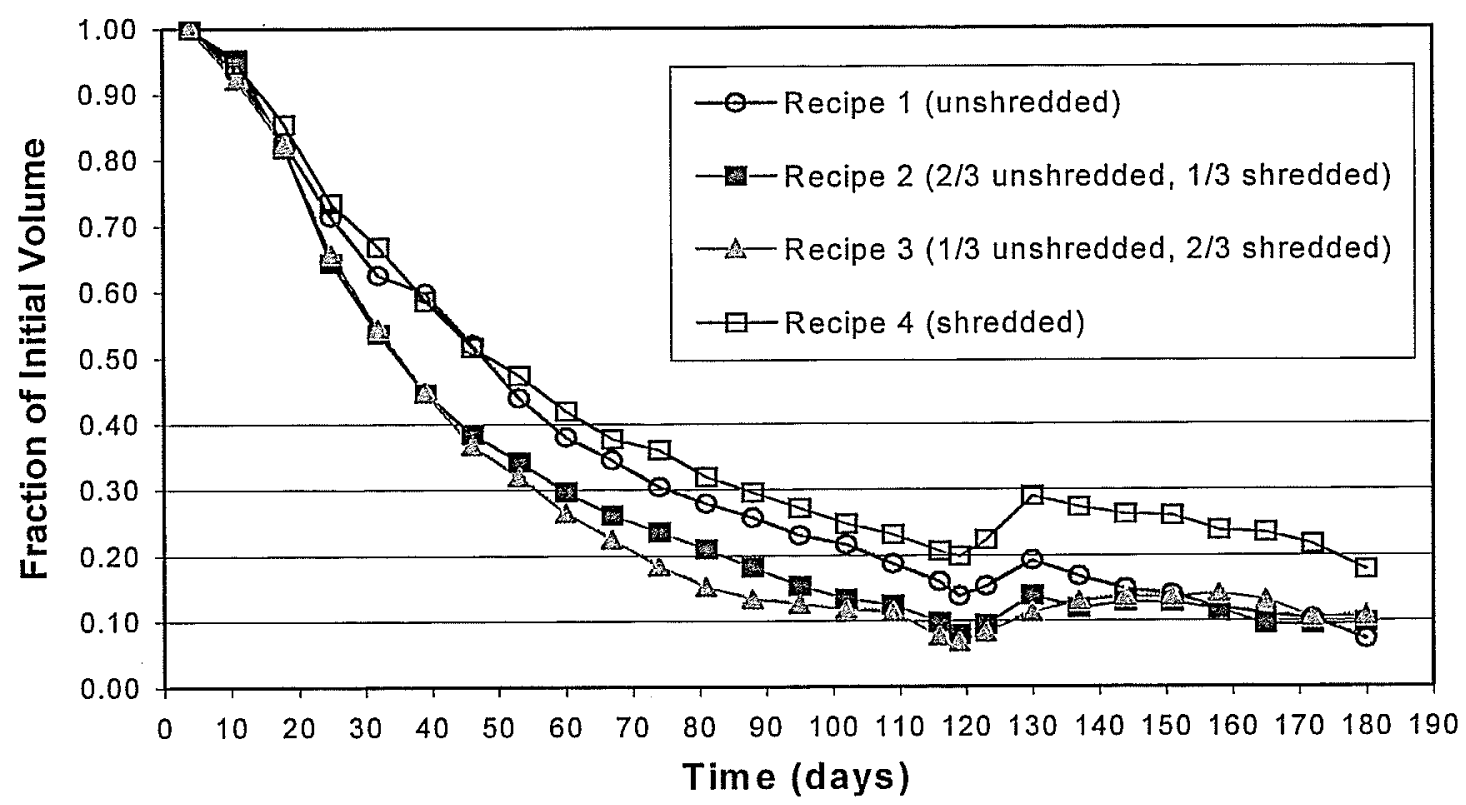

Figure 5.2: Volume reductions for Phase 2 recipes.

Note: Results indicate averages of duplicate reactors.

\subsubsection{Temperature and Oxygen}

Temperature monitoring for each compost reactor continued until day 56, at which point a plateau in the temperature profile was evident as shown in Figure 5.3. It was noticed that the diminished mass of compost remaining in each reactor at this time might not have been capable of heating itself to a temperature greater than that within the environmental chamber. The average temperature within the environmental chamber was 42. $6^{\circ} \mathrm{C}$. Epstein (1997) reported on the work of others who studied the succession of fungi in straw and grass compost. They found that both mesophilic and thermophilic fungi decreased in population as temperature of compost reached $70^{\circ} \mathrm{C}$. However, as soon as the temperature decreased below $65^{\circ} \mathrm{C}$, the thermophilic fungi resumed growth and their population peaked at approximately $45^{\circ} \mathrm{C}$. 


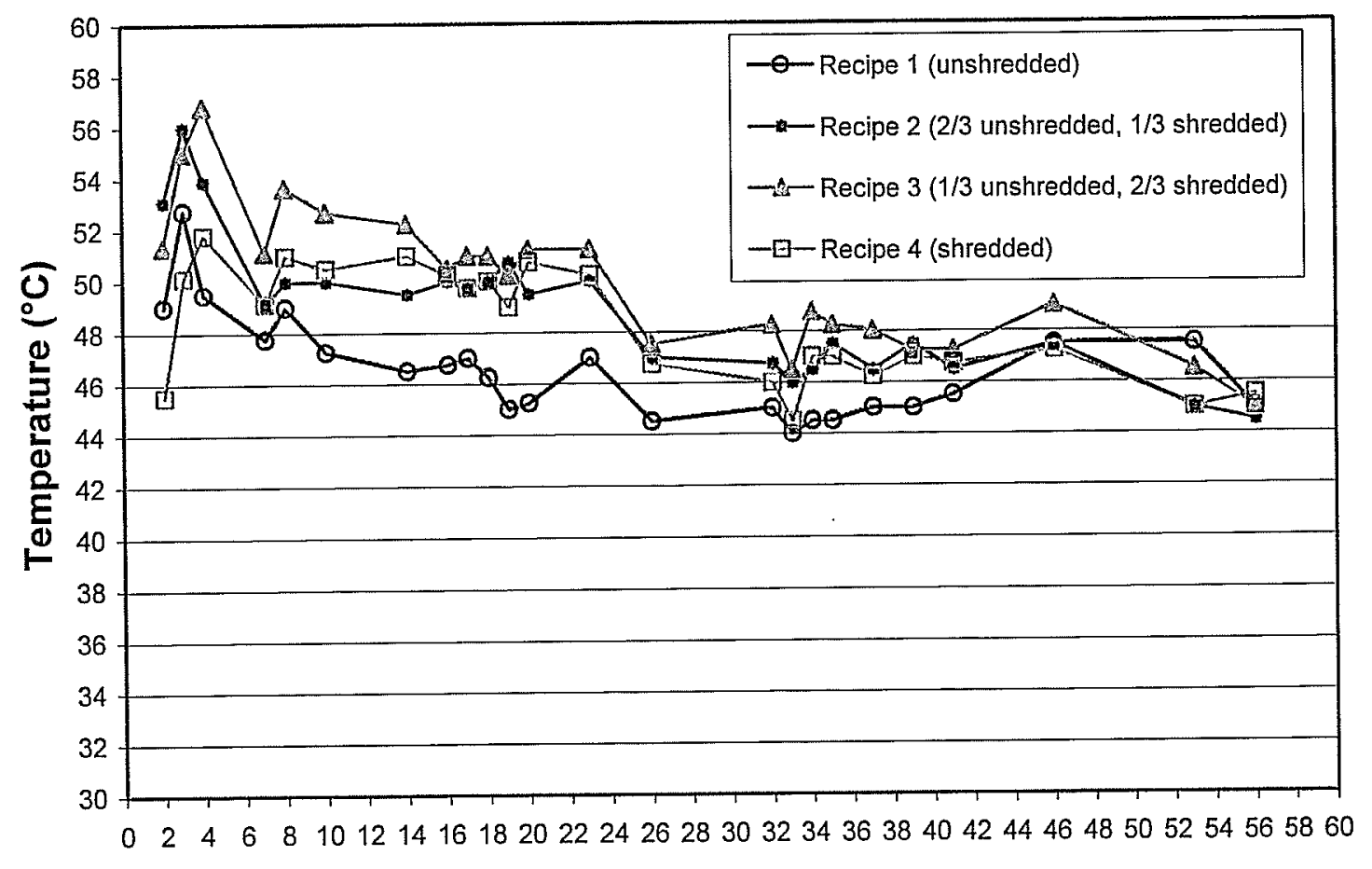

Day

Figure 5.3: Temperature measurements for Phase 2 recipes.

Note: Results indicate averages of duplicate reactors.

The temperature rise in actively composting wastes is a function of both the heat gain from microbial exothermic reactions associated with respiratory metabolism (Tchobanoglous et al. 1993; Epstein, 1997), and heat loss due to ventilation. Recipe 3 exhibited the highest temperatures throughout the monitoring period perhaps indicating the highest level of microbial activity and/or least heat loss. Temperature maxima were reached on day 4 for all recipes and gradually decreased throughout the monitoring period. Average temperatures for Recipes $1,2,3$, and 4 were $46.5^{\circ} \mathrm{C}, 48.9^{\circ} \mathrm{C}, 50.0^{\circ} \mathrm{C}$, and $48.3^{\circ} \mathrm{C}$ for the monitoring period, respectively.

Oxygen levels within the compost reactors were only monitored from day 13 to day 20 due to measuring probe malfunctions. The contents of each reactor were mixed on day 18 restoring the pore space within the compost matrix. Average oxygen levels (\%) 
throughout the monitoring period for Recipes $1,2,3$, and 4 were 17.5, 12.9, 10.0, and 8.9, respectively. These results were expected since the supply of oxygen to compost microorganisms depends on the porosity and particle size within the compost, its moisture content and rates of diffusion and convection, the latter two being affected by temperature (Joshua et al. 1998). As moisture content and temperature were constants for all compost recipes, the particle size variations for Recipe 1 through 4 directly impacted oxygen diffusion and convection throughout the compost matrix. All recipes exhibited pore space oxygen levels above the minimum oxygen concentration of $5 \%$ suggested by Rynk (1992) to assure efficient aerobic metabolism.

\subsubsection{Volatile Solids Reduction}

A plot of volatile solids reduction for each recipe throughout the duration of the experiment is shown in Figure 5.4. Volatile solids reduction was most significant and rapid for Recipe 3 decreasing from $88 \%$ to a final value of $58 \%$ while Recipes 1, 2 and 4 showed volatile solids reduction to final values of $63 \%, 61 \%$, and $65 \%$, respectively. Volatile solids began to stabilize after approximately 140 days. Comparatively, Eiland et al. (2001) evaluated the composting of Miscanthus straw and liquid pig manure in both open box and closed reactor systems. VS in the box system decreased from $75 \%$ to $68 \%$. In the reactor system, VS decreased from $78 \%$ to $65 \%$. VS stabilization was observed at approximately day 120 in both systems. The VS reductions in the experiment reported herein ranged from 25 to $30 \%$ whereas the VS reductions in the Eiland et al. (2001) experiment ranged from 7 to $13 \%$. However, moisture content in the box and reactor systems in the Eiland et al. (2001) experiment fluctuated as low as 53\% in the box system with final moisture contents of $80 \%$ for both systems. Also, temperatures in that 
experiment varied from $16^{\circ} \mathrm{C}$ to maxima of $65-70^{\circ} \mathrm{C}$. The conditions in the present experiment may have been better suited for straw composting. Temperature was stable within the $40-50^{\circ} \mathrm{C}$ range (ideal for thermophilic fungi) and moisture content was controlled at $70 \%( \pm 5 \%)$.

Volatile solids degradation in Recipe 1 may have been slowed by a larger initial particle size that inhibited microbial activity. Conversely, the smaller particle sizes (and pore spaces) in Recipe 4 coupled with a high moisture content of $70 \%$ may have produced oxygen constraints which limited oxygen transport (McCauley and Shell, 1956; Miller, 1991; Hamelers, 1992; Tseng et al. 1995; Richard, 1996) and reduced the rate of decomposition (Richard et al. 1999). Pore space oxygen measurements from day 13 to day 20 seem to indicate that oxygen levels were adequate $(>5 \%)$, however, the one week of measurements only represented a small fraction of the nearly 26-week composting period. 


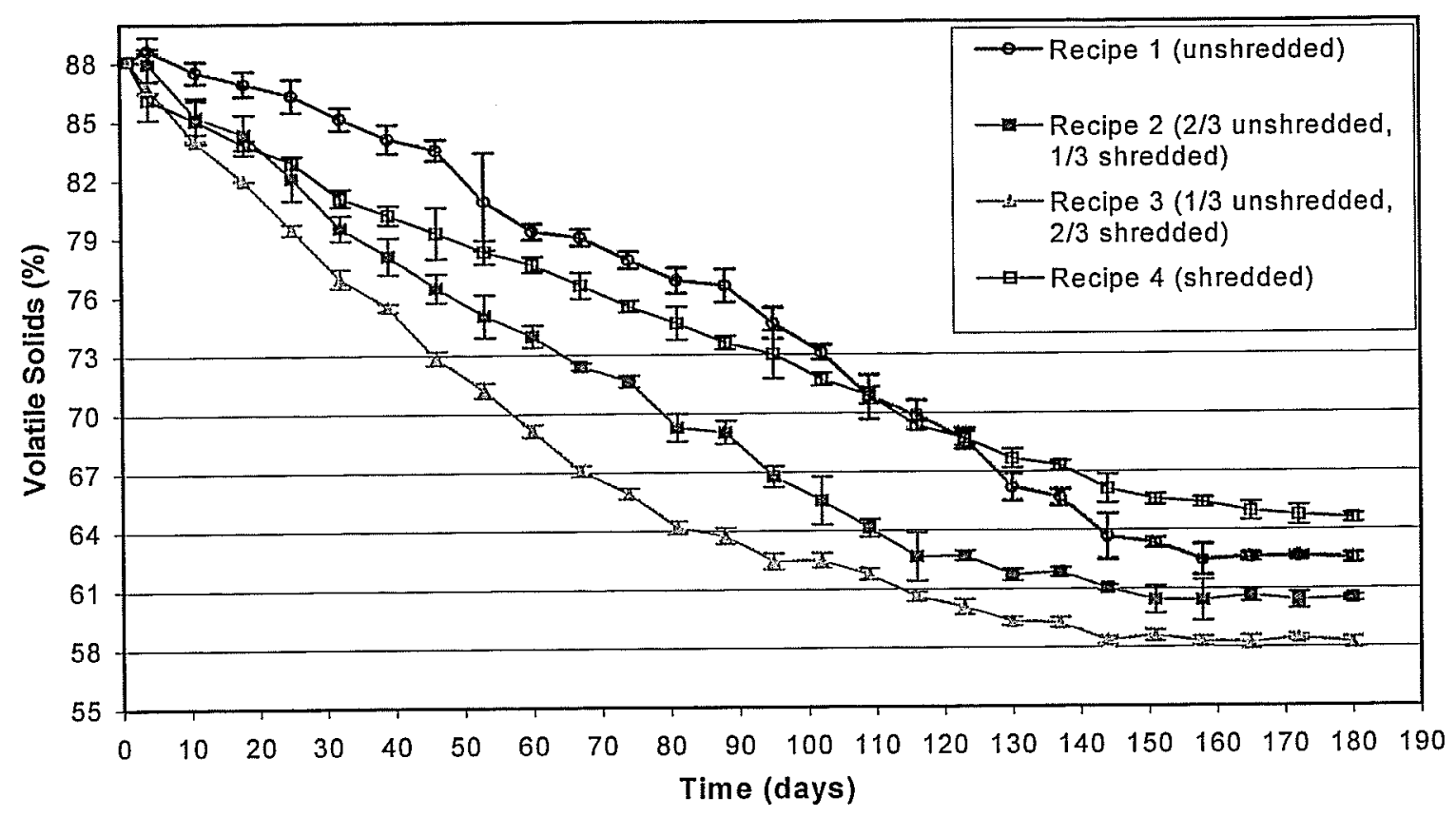

Figure 5.4: Volatile solids reduction for Phase 2 recipes.

Note: Results indicate averages of duplicate reactors. Error bars represent \pm one standard deviation of recipe duplicates.

\subsubsection{Fibre Degradation}

The degradation of straw fibres (hemicellulose, cellulose, and lignin) during composting is shown in Figures 5.5, 5.6, and 5.7. Since the homogeneity of the composting material increased (due to decreasing particle size) over the composting period, the within recipe variability decreased with time, as indicated by the error bars presented in Figures 5.5 to 5.7. Additionally, Figure 5.5, showing the degradation of hemicellulose, appears to dip below zero on the y-axis. The reason for this may be due to the analytical method used in this experiment. Many complex polysaccharides that can be classed as hemicelluloses are water-soluble and will thus not be estimated by this method. Secondly, some hemicelluloses require a $\mathrm{pH}$ of less than zero to be solubilised and thus the ADF assay will not remove all of them. Generally, fibre degradation was most rapid and complete for the compost recipes containing mixtures of unshredded and shredded straw. Recipe 3 
performed the best, followed closely by Recipe 2 . Recipes 1 and 4 showed the slowest rates of degradation.

Hemicellulose decreased from $1.72 \mathrm{~g} \mathrm{~g}^{-1}$ ash to zero by day 90 for Recipes 2, 3, and 4 and by day 110 for Recipe 1 . While hemicellulose was eliminated in this experiment, Eiland et al. (2001) observed that hemicellulose content only decreased to $6 \%$ of the initial hemicellulose content at the end of the composting period (190 days in the box system, 150 days in the closed reactor).

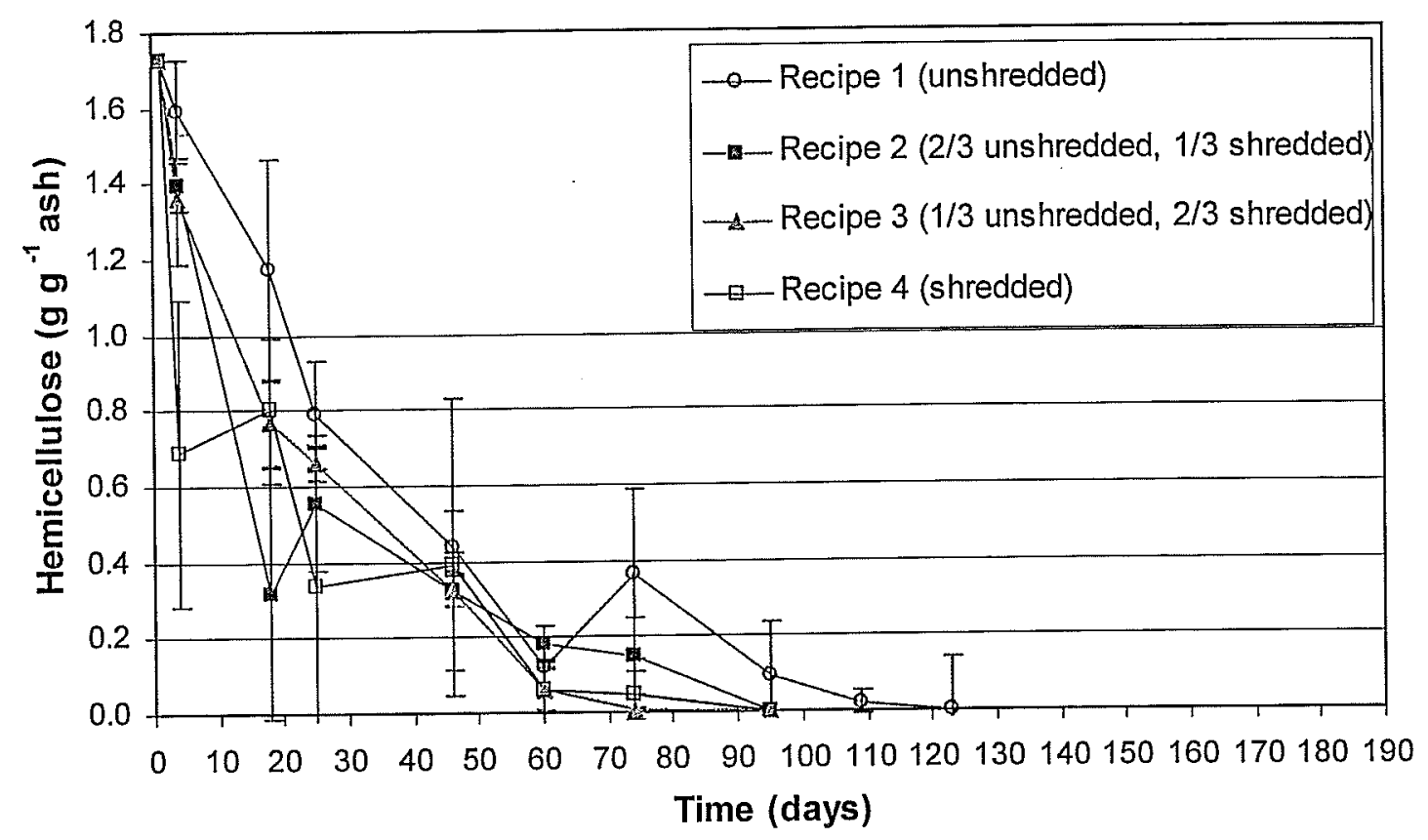

Figure 5.5: Hemicellulose degradation during Phase 2 composting.

Note: Results indicate averages of duplicate reactors. Error bars represent \pm one standard deviation of recipe duplicates.

In the experiment by Eiland et al. (2001), cellulose reached stable levels at day 50 . Cellulose final levels of $36 \%$ and $30 \%$ of initial cellulose levels were determined for the box and reactor systems respectively. As stated previously in the discussion of VS reduction, the conditions in the present experiment may have been better suited for straw composting. The stabilization of cellulose was most rapidly achieved by Recipe 3 (day 
60), followed by Recipe 2 (day 95), and Recipes 1 and 4 (day 137). By day 137, cellulose content had decreased from $3.75 \mathrm{~g} \mathrm{~g}^{-1}$ ash to $0.75 \mathrm{~g} \mathrm{~g}^{-1}$ ash for all recipes representing a loss of $80 \%$.

Additionally, work reported by Epstein (1997) determined that after 153 days of composting, straw had lost approximately $33 \%$ of the hemicellulose and $50 \%$ of the cellulose initially present. No change in cellulose levels was observed before day 24 and the most rapid degradation occurred from day 24 to day 90 . Results from the present experiment show that rapid degradation of hemicellulose and cellulose began early in the composting period (by day 4) and continued until approximately day 60 .

Eklind (1998) evaluated the degradation of the fibre fraction as half-life times (50\% degradation). In that evaluation, straw composting exhibited half-life times for hemicellulose and cellulose of 29 and 26 days respectively. Eiland et al. (2001) observed half-life times for hemicellulose and cellulose of 21 and 100 days respectively. Results for this study were similar to the straw-only composting in Eklind's (1998) experiment with half-life times for hemicellulose of approximately 20 days for all recipes, and cellulose half-life times of 20 days for Recipes 2, 3, and 4 and 50 days for Recipe 1.

Epstein (1997) reported on the biochemical changes that occur during the composting of wheat straw. Cellulose and hemicellulose, constituting $45.3 \%$ and $35.7 \%$ of the initial dry weight, decreased to $13.3 \%$ and $17.0 \%$ of the original dry weight in 60 days. Also, the straw had lost $50 \%$ of its initial dry weight after 60 days of composting, essentially representing the loss of hemicellulose and cellulose. In the present study, cellulose and hemicellulose decreased from $42.5 \%$ and $19.7 \%$ of the initial dry weight to $22-30 \%$ and $0.5-5 \%$, respectively after 60 days. All recipes had lost $50 \%$ of the initial dry weight by 
the $90^{\text {th }}$ day of composting. After 180 days, cellulose had decreased to $30 \%$ of the initial dry weight while hemicellulose had been completely degraded.

The microbial enzymes catalyzing degradation of hemicellulose and cellulose are repressed by the presence of low-molecular weight carbon sources that are more easily metabolized than hemicellulose and cellulose (Madigan et al. 2000). This explanation supports results obtained by Eiland et al. (2001) where, while composting straw with liquid pig manure, the metabolism of readily available carbon creating high metabolic rates resulted in a period of high temperature where hemicellulose and cellulose degradation was delayed until the eighth day. However, hemicellulose and cellulose degradation is noticed earlier for this experiment (day 4), indicating a lack of more easily degraded carbon.

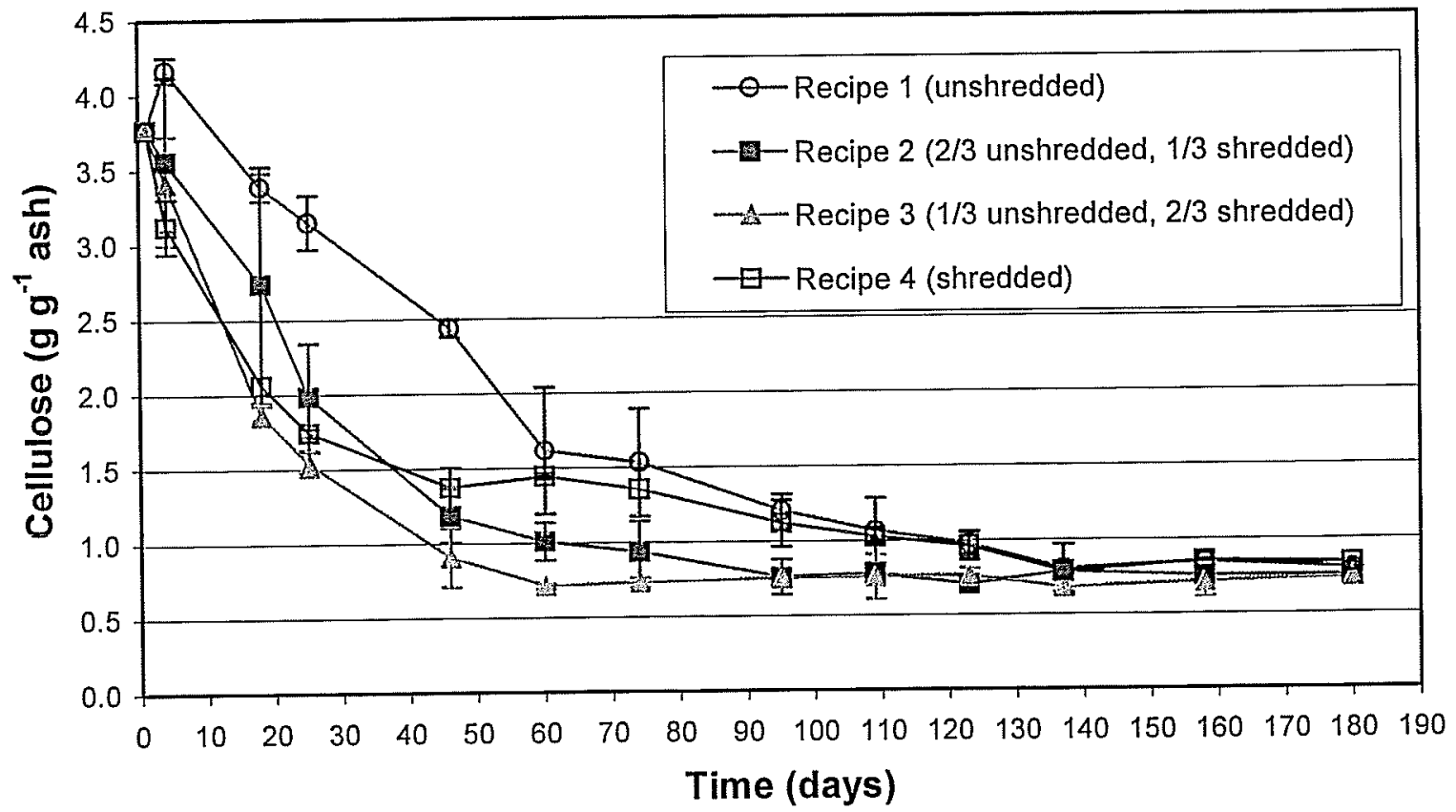

Figure 5.6: Cellulose degradation during Phase 2 composting.

Note: Results indicate averages of duplicate reactors. Error bars represent \pm one standard deviation of recipe duplicates. 
The degradation of lignin was slower and less complete than hemicellulose and cellulose degradation in this experiment. Lignin content decreased from $1.0 \mathrm{~g} \mathrm{~g}^{-1}$ ash to $0.4 \mathrm{~g} \mathrm{~g}^{-1}$ ash for Recipes 2 and 3, $0.5 \mathrm{~g} \mathrm{~g}^{-1}$ ash for Recipe 1, and $0.6 \mathrm{~g} \mathrm{~g}^{-1}$ ash for Recipe 4. Lignin levels were approximately stable by day 140 . In the work done by Eiland et al. (2001), no degradation of lignin was observed. It was hypothesized that this was caused by the presence of nitrogen in the final stage of the composting period, because lignin-degrading enzymes are induced by nitrogen limitation. The initial C:N ratios were 25 and 16 for box and reactor systems respectively. The initial C:N ratio for Phase 2 of this study was 64.2, perhaps creating more ideal nitrogen-limiting conditions for lignin degradation.

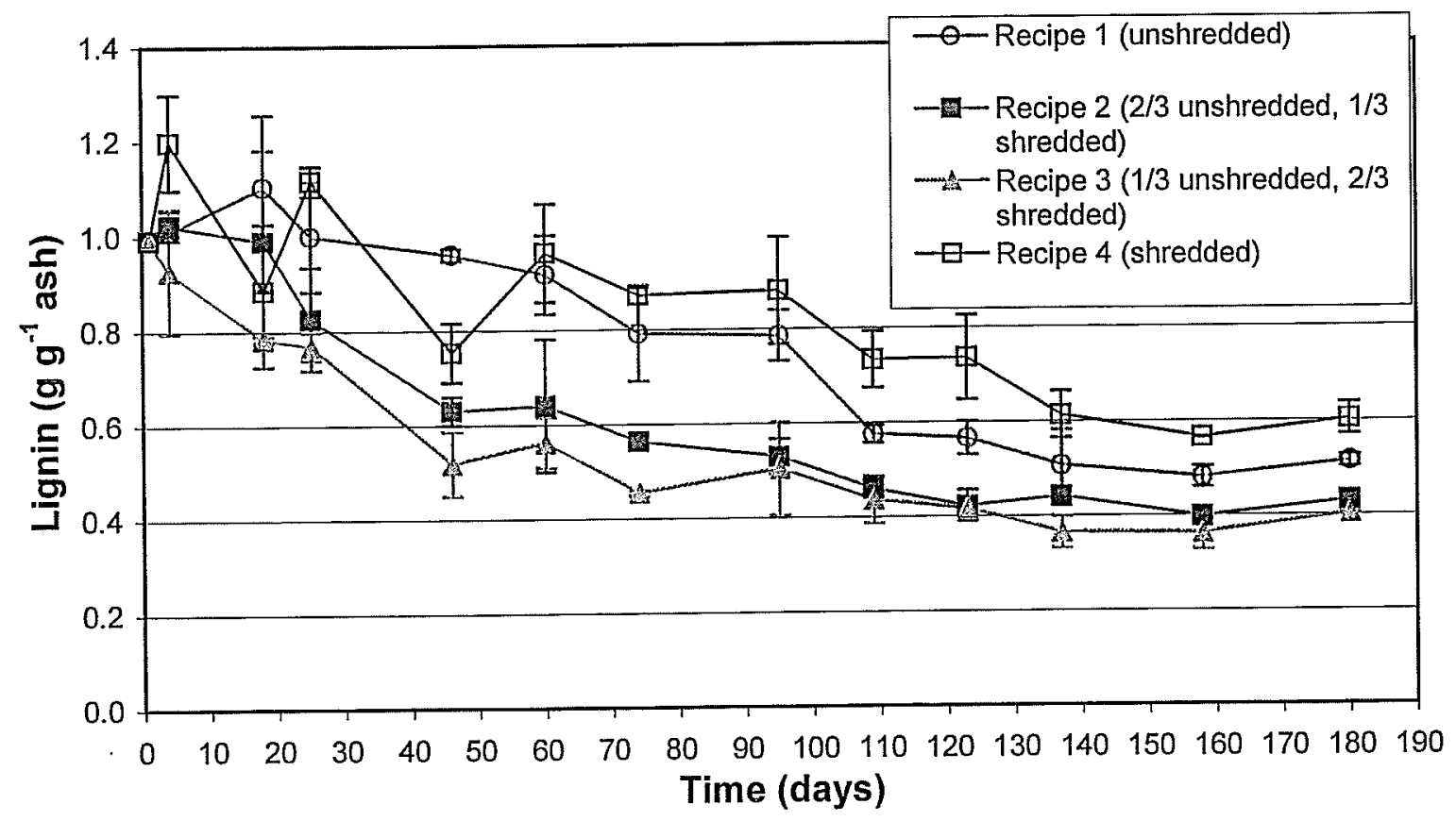

Figure 5.7: Lignin degradation during Phase 2 composting.

Note: Results indicate averages of duplicate reactors. Error bars represent \pm one standard deviation of recipe duplicates. 


\subsection{SUMMARY AND CONCLUSIONS}

Phase 1 of the research was a preliminary investigation that employed small bench-scale compost reactors containing various recipes using the three feedstock materials (straw, process unders, and lagoon mixture) at different moisture levels in an attempt to gain an understanding of composting effectiveness and to aid in the selection of several recipes that could be used for larger-scale laboratory testing. The recipe variations used formed the basis for comparing the impacts of: (1) water addition; (2) unders addition; and (3) nitrogen addition in the form of a lagoon mixture. The Phase 1 small bench-scale results for this project indicated:

1) The VS reduction (indicating degradability) was greater for the compost recipes containing unders suggesting the possibility that unders, having a smaller particle size and thus a larger surface area, are more easily biodegraded.

2) A clear trend emerged indicating that VS reduction was aided by an increase in moisture content for the straw and straw/unders recipes.

3) The advantages of adding a nitrogen source (in the form of a lagoon mixture) were limited when considering VS reduction. The $\mathrm{N}$ addition also resulted in a greater loss of $\mathrm{N}$ throughout the experiment. No trend emerged with respect to $\mathrm{N}$ loss with increasing moisture content for the straw/unders recipes.

Phase 2 was a larger-scale investigation focused on the composting of four recipes of varying straw particle sizes. The objectives for this phase were to investigate the effects of particle size on compost degradation and volume reduction and to monitor the rate and degree of degradation over a typical Canadian Prairie summer composting period to estimate the ultimate degradability of straw compost. Based on the analysis of the observed data the following conclusions were made: 
1) Degradation of volatile solids and lignocelluloses was most rapid and complete for recipes containing both shredded and unshredded straw, with the recipe containing $2 / 3$ shredded straw performing the best.

2) Volume reduction reached approximately $90 \%$ for recipes containing either unshredded straw only or a mixture of unshredded and shredded straw. 


\subsection{RECOMMENDATIONS AND ENGINEERING SIGNIFICANCE}

The conclusions reached as a result of Phase 1 and Phase 2 of this experiment offer insight for a potential full-scale operation attempting to compost a high carbon material such as straw. The rates and degree of degradation as well as volume reduction during straw composting are of great importance for the sizing and operation of a full-scale facility. In addition, understanding the impacts of straw particle size could result in potential cost savings with respect to feedstock preparation via straw shredding.

However, this research consisted of laboratory-controlled experiments to simulate windrow composting of straw over a typical Canadian Prairie summer. Parameters such as moisture level, humidity, temperature, and compost mixing can be controlled and optimized in the laboratory, but challenges exist in replicating laboratory conditions at a larger field scale. As a logical next step towards full-scale design, a pilot-scale field trial would be useful in determining larger-scale composting conditions and, if possible, provide correlations with the results of the completed laboratory-scale work. 


\subsection{REFERENCES}

APHA 1995. "Standard methods for the examination of water and wastewater", American Public Health Association, American Wastewater Association, Waste Environment Federation, $19^{\text {th }}$ Edition.

ASTM, 1990. "Standard test method for particle-size analysis", D 422-63. Annual Book of ASTM Standards. Volume 04.08 Soil and Rock. ASTM, Philadelphia, PA, USA.

Basaglia, M., G. Concheri, S. Cardinali, M.B. Pasti-Grigsby, and M.P. Nuti. 1992.

"Enhanced degradation of ammonium-pretreated wheat straw by lignocellulolytic Streptomyces spp.", Canadian Journal of Microbiology, Vol. 38, No. 10, pp. 1022-1025.

Carter, M.R. 1993. "Soil sampling methods of analysis", Lewis Publishers, Boca Raton, Florida, USA.

Crawford, D.L. 1986. "The role of actinomycetes in the decomposition of lignocellulose", FEMS Symposiium, 34, pp. 715-728.

Eftoda, G. and D. McCartney, 2002. "Correlating bench-scale FAS tests to full-scale windrow composting performance", Proceedings of 2002 International Symposium on Composting and Compost Utilization. Columbus, Ohio, USA.

Eiland, F., M. Leth, M. Klamer, A.-M. Lind, H.E.K. Jensen, and J.J.L. Iversen, 2001. "C and $\mathrm{N}$ turnover and lignocellulose degradation during composting of miscanthus straw and liquid pig manure", Compost Science and Utilization, Vol. 9, No. 3, pp. 186-196.

Epstein, E. 1997. "The science of composting", Technomic Publishing Co., Lanchester, PA, USA.

Hamelers, H.V.M. 1992. "A theoretical model of composting kinetics", In: H.A.J. Hoitink and $\mathrm{H}$. Keener (eds.). Science and Engineering of Composting. Columbus, $\mathrm{OH}$, Renaissance Publications, pp. 36-58.

Haug, R.T. 1993. "The practical handbook of compost engineering", Lewis Publishers. CRC Press, Inc., Boca Raton, Florida, USA.

Joshua, R.S., B.J. Macauley, and H.J. Mitchell. 1998. "Characterization of temperature and oxygen profiles in windrow composting", Compost Science and Utilization, Vol. 6, No. 4, pp. 15-28.

Klute, A. et al. 1986. "Methods of soil analysis", American Society of Agronometry, Inc., Soil Science Society of America Inc., Madison, Wisconsin, USA. 
Komarek, A.R., J.B. Robertson, and P.J. van Soest, 1993. "A comparison of methods for determining $\mathrm{ADF}$ using the filter bag technique versus conventional filtration", Proceedings of the National Conference on Forage Quality, Evaluation and Utilization. Journal of Dairy Science, 77(suppl. 1).

Komarek, A.R., J.B. Robertson, and P.J. van Soest, 1994. "Comparison of the filter bag technique to conventional filtration in the van Soest NDF analysis of 21 feeds", Proceedings of the National Conference on Forage Quality, Evaluation and Utilization. Abstracts, p. 78, University of Nebraska, Lincoln, NE, USA.

Ladisch, M.R., K.W. Lin, M. Voloch, and G.T. Tsao, 1983. "Process considerations in the enzymatic hydrolysis of biomass", Enzyme Microbial Technology, Vol. 5, No. 2, pp. 82-102.

Larson, K.L. and D.M. McCartney, 2000. "Effect of C:N ratio on microbial activity and $\mathrm{N}$ retention: bench-scale study using pulp and paper biosolids", Compost Science and Utilization, Vol. 8, No. 2, pp. 147-159.

Liao, P.H., A.T. Vizcarra, A. Chen, and K.V. Lo. 1995. "A comparison of different bulking agents for the composting of fish offal", Compost Science and Utilization, Vol. 3, No. 1, pp. 80-86.

Lynch, J.M. 1992. "Substrate availability in the production of composts", Proceedings of the International Composting Research Symposium, H.A.J. Hoitink and H. Keener (Editors). pp. 24-35.

McCartney, D. and H. Chen, 2001. "Using a biocell to measure effect of compressive settlement on free air space and microbial activity in windrow composting", Compost Science and Utilization, Vol. 9, No. 4, pp. 285302.

McCartney, D. and J. Tingley, 1998. "Development of a rapid moisture content method for compost materials", Compost Science and Utilization, Vol. 6, No. 3, pp. 14-25.

McCauley, R.F. and B.J. Shell. 1956. "Laboratory and operational experiences in composting", Proceedings of the $11^{\text {th }}$ Industrial Waste Conference, Purdue Univ. Eng. Bull. Series No. 91, pp. 436-453.

Miller, F.C. 1991. "Biodegradation of solid wastes by composting", In: A.M. Martin (ed.). Biological Degradation of Wastes. Elsevier Applied Science, London, pp. 1-31. 
Richard, T.L. and L.P. Walker, and J.M. Gossett, 1999. "The effects of oxygen on solidstate biodegradation kinetics", Proceedings of the Inst. of Biological Engineering 2: A10-A30. IBE Publications, Athens, GA, USA.

Richard, T.L., 1996. "Oxygen transport" [Online], Available: $\mathrm{http}: / /$ www.cfe.cornell.edu/compost/oxygen/oxygen.transport.html. [March 5 2002].

Rykens, B.A., 1977. "Some possible uses of straw", Report on Straw Utilization Conference, Feb. 24-25, 1977, Oxford, England, UK.

Rynk, R. 1992. "On farm composting handbook", NRA\#S-54. Northeast Regional Agricultural Engineering Service, Ithaca, New York, USA.

Tchobanoglous, G., H. Theisen, and S. Vigil. 1993. "Integrated solid waste management, engineering principles and management issues", McGraw-Hill Inc., Toronto, Ontario, Canada.

Tseng, D.Y., J.J. Chalmers, O.H. Tuovinen and H.A.J. Hoitink. 1995. "Characterization of a bench-scale system for studying the biodegradation of organic solid wastes", Biotechnol. Progr. Vol. 11, pp. 443-451.

USCC. 1997. "Test methods for the examination of composting and compost, first edition", The U.S. Composting Council.

Van Soest, P.J. 1963. "Use of detergents in the analysis of fibrous feeds", Journal of Association of Official Analytical Chemists, Issue 46, pp. 825-835.

Wizbicki, R. 2002. "Effect of biocell diameter on the measurement of physical behavior of wood chips", Engineering Undergraduate Thesis, University of Manitoba, Winnipeg, Manitoba, Canada. 
APPENDIX A - PHASE 1 COMPOSTING PHOTOGRAPHS 


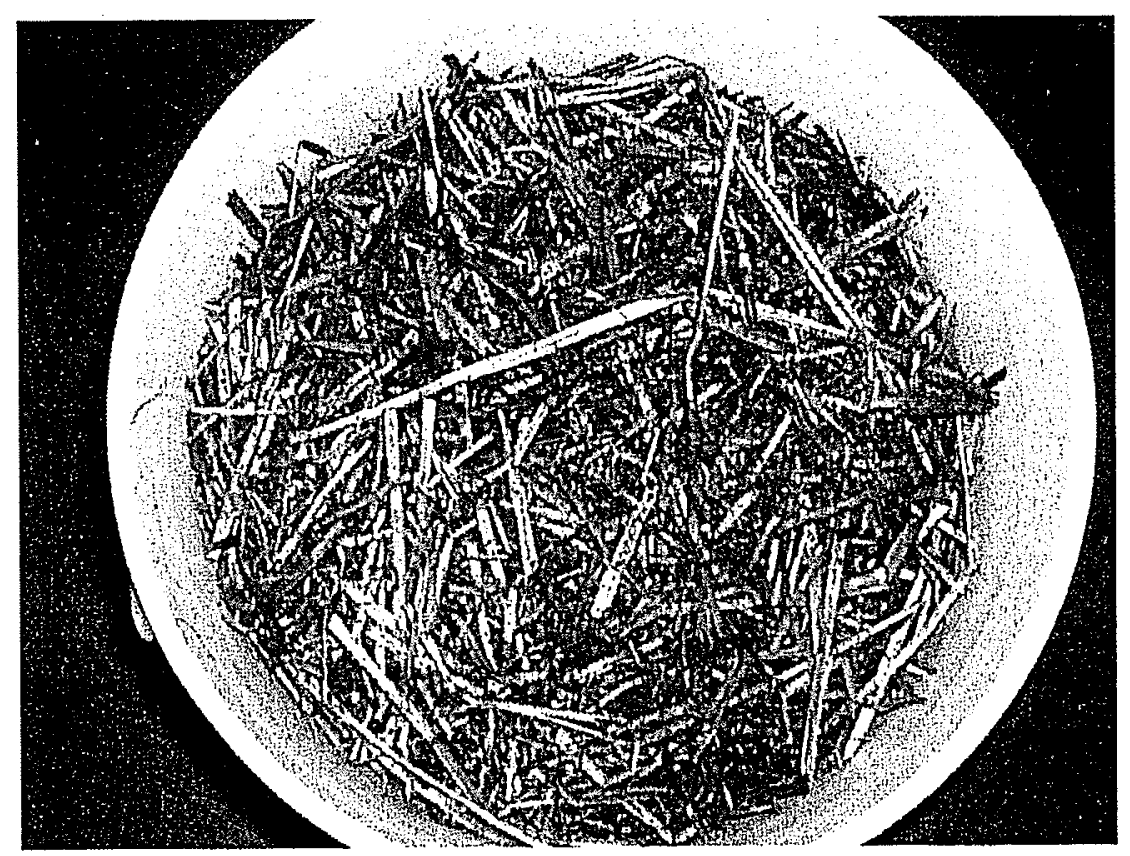

Figure A-1: Phase 1 composting, initial Recipe 1.

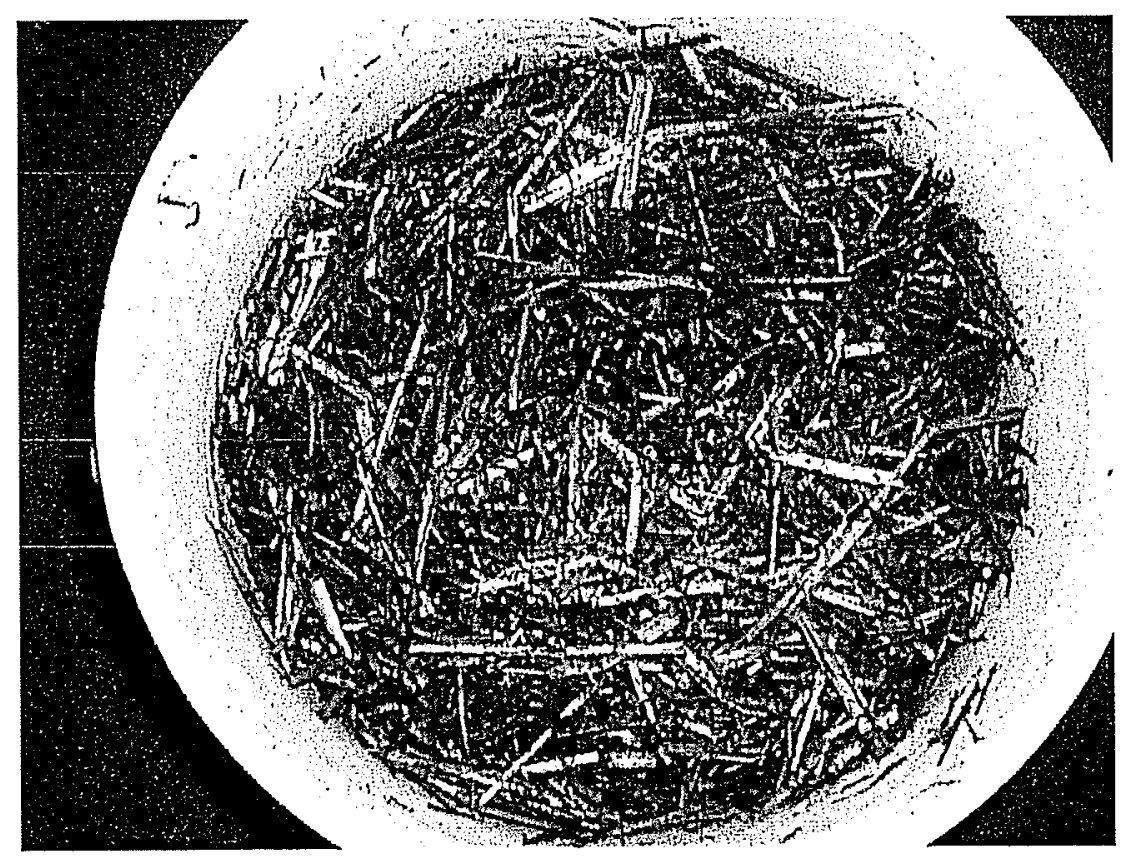

Figure A-2: Phase 1 composting, initial Recipe 2. 


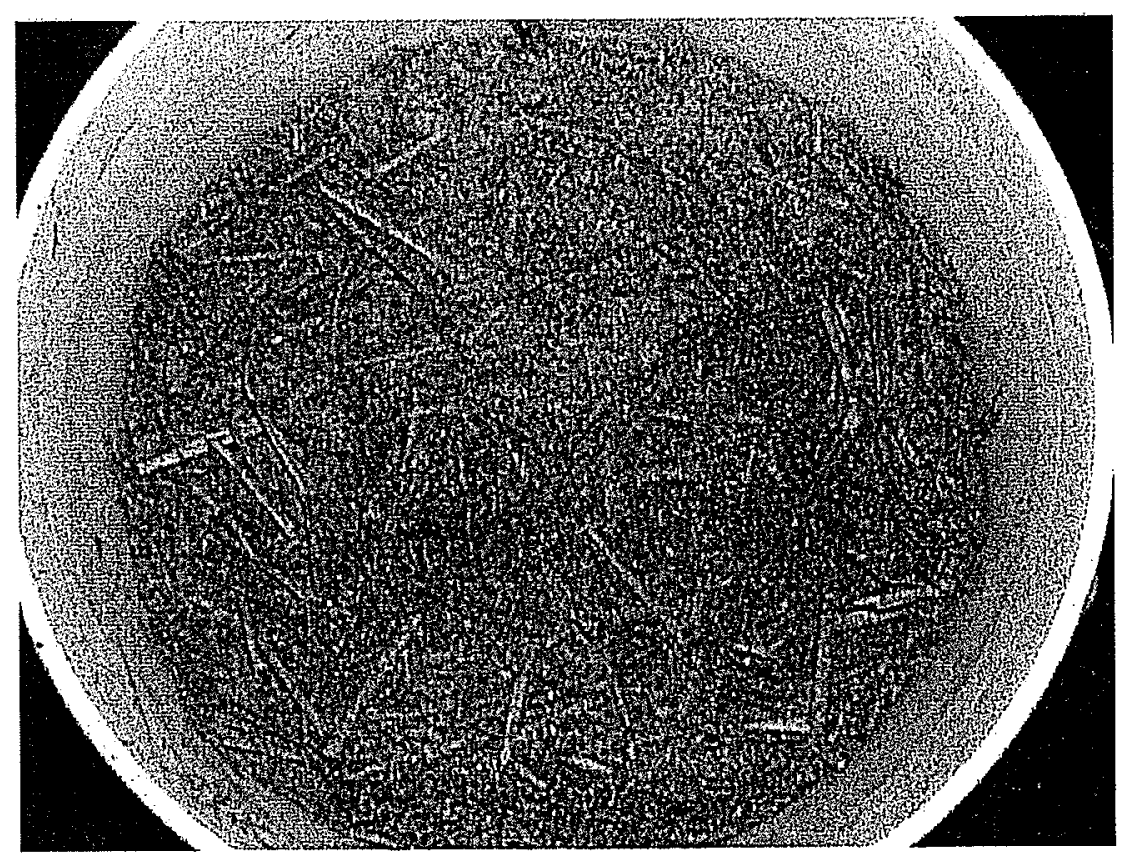

Figure A-3: Phase 1 composting, initial Recipe 3.

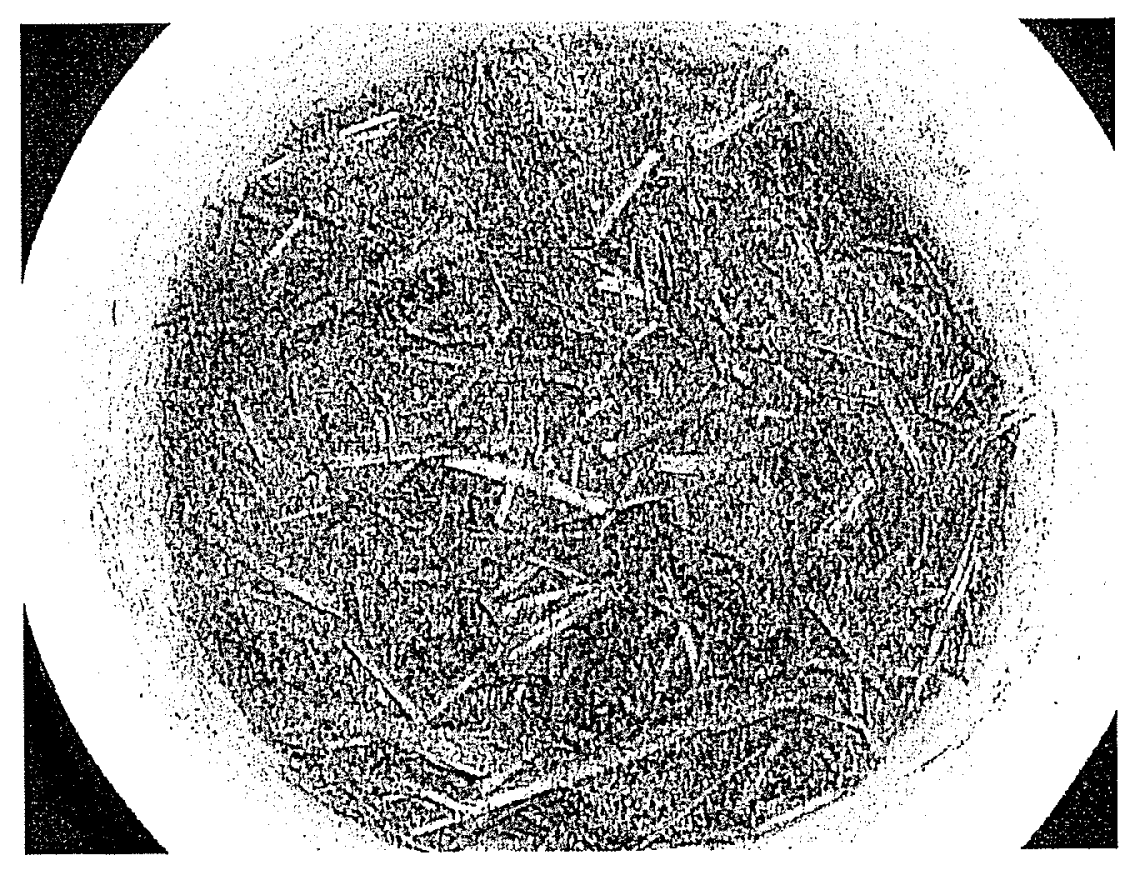

Figure A-4: Phase 1 composting, initial Recipe 4. 


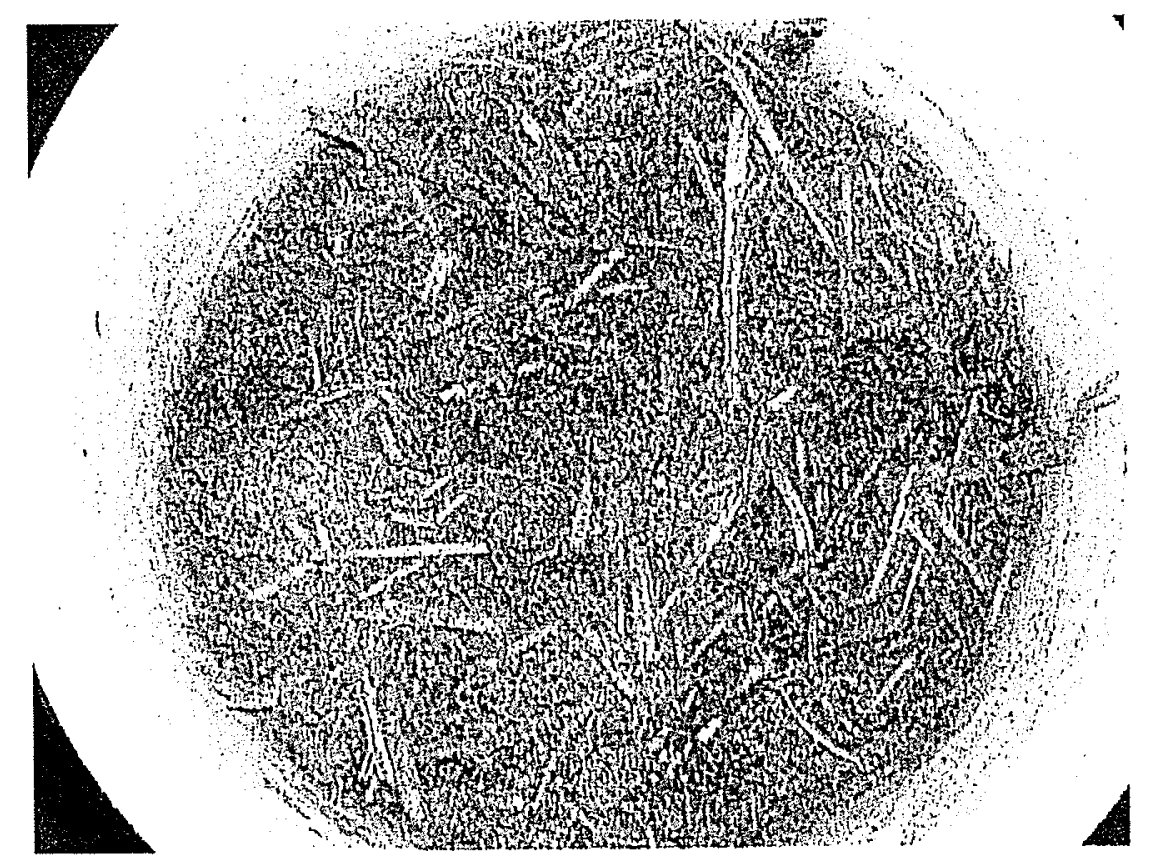

Figure A-5: Phase 1 composting, initial Recipe 5.

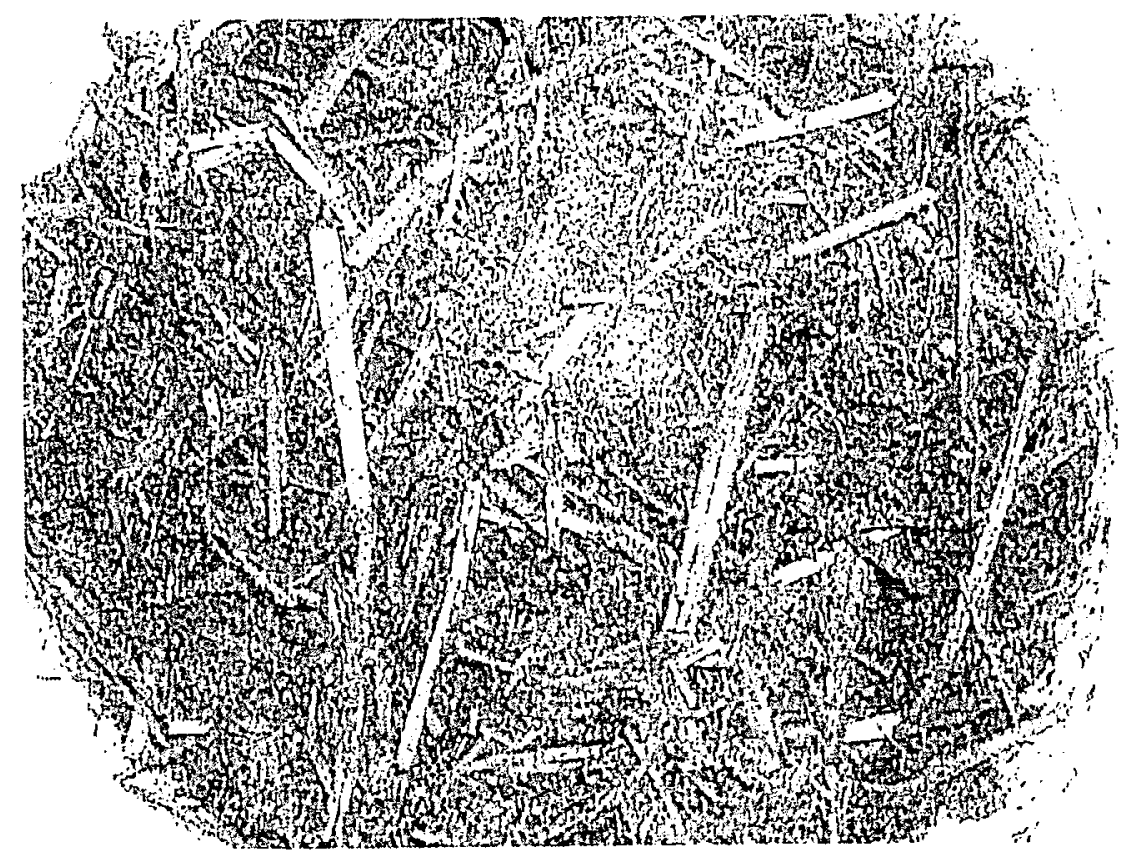

Figure A-6: Phase 1 composting, initial Recipe 6. 


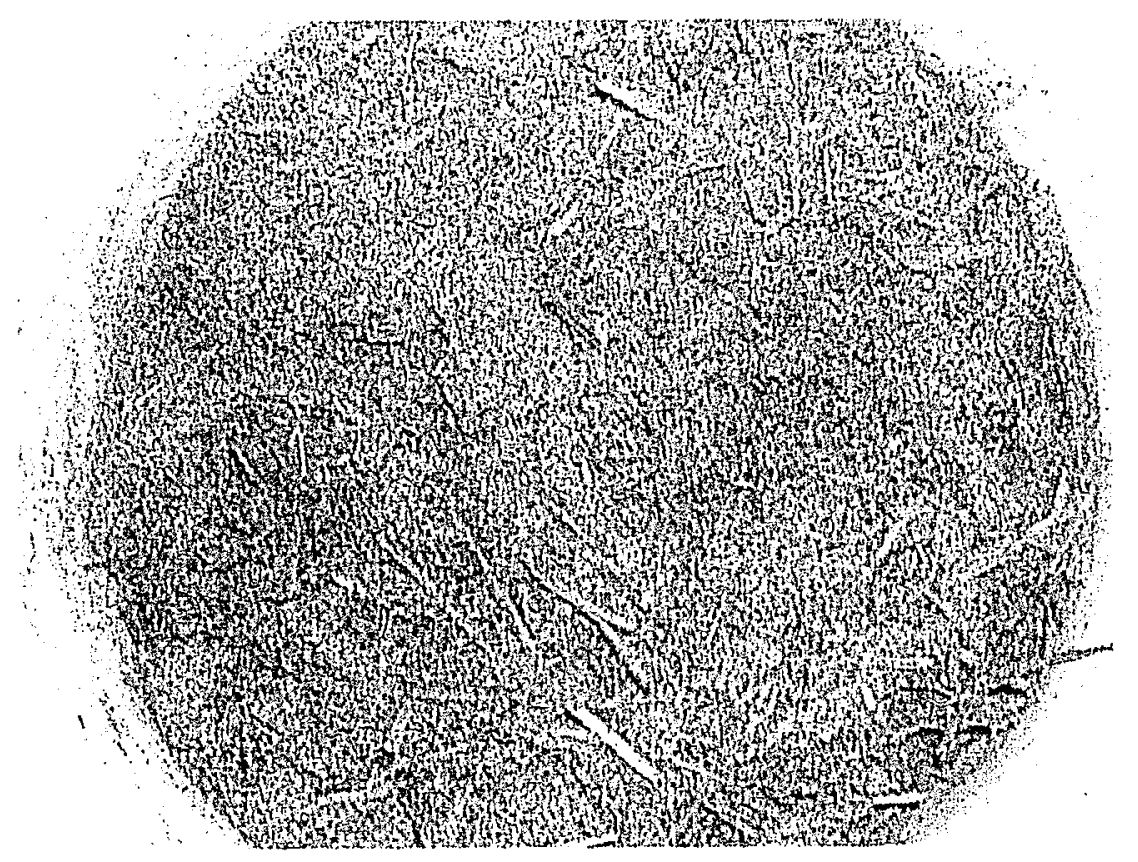

Figure A-7: Phase 1 composting, initial Recipe 7.

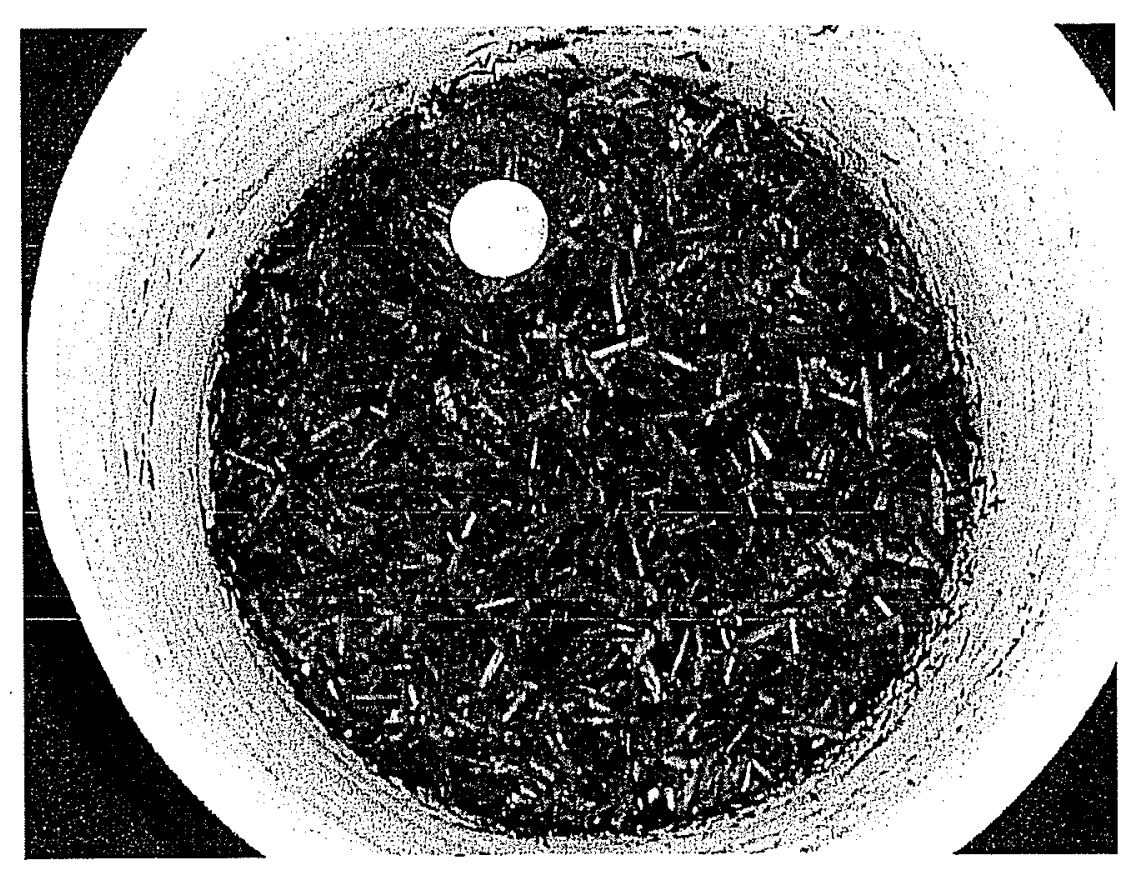

Figure A-8: Phase 1 composting, finished Recipe 1. 


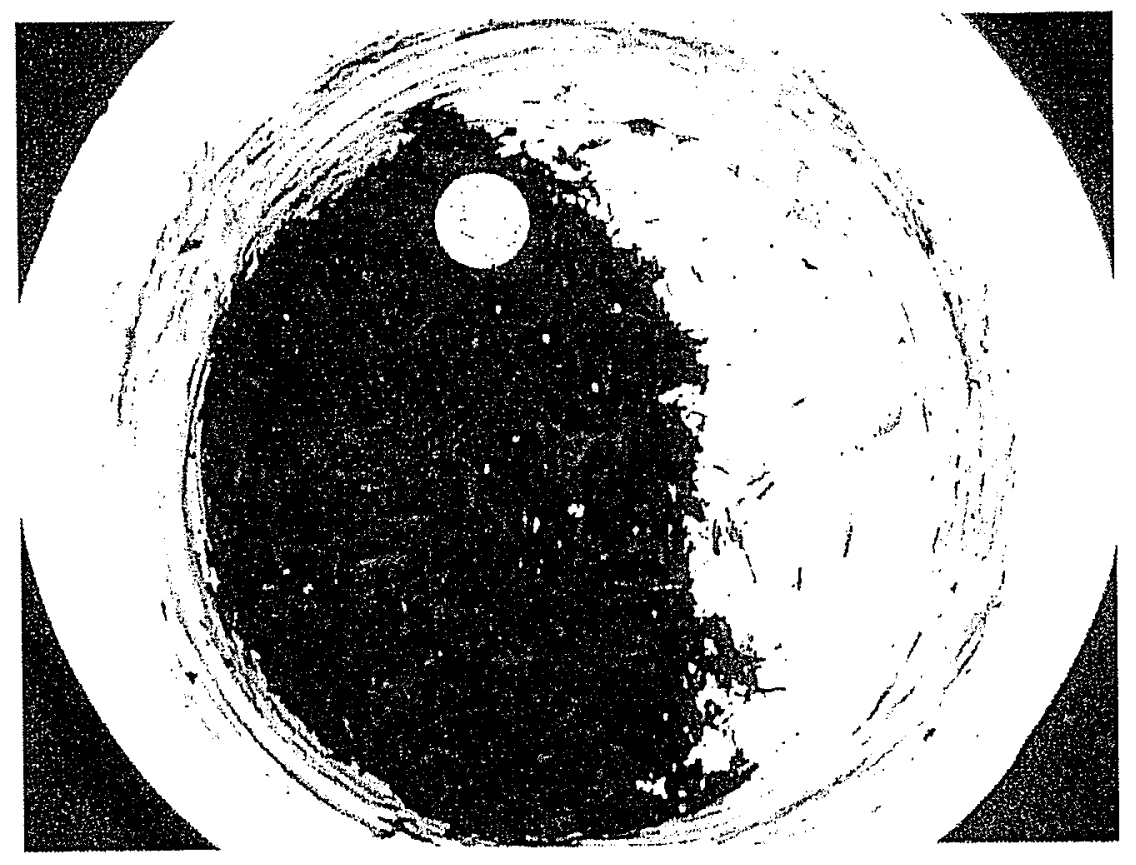

Figure A-9: Phase 1 composting, finished Recipe 2.

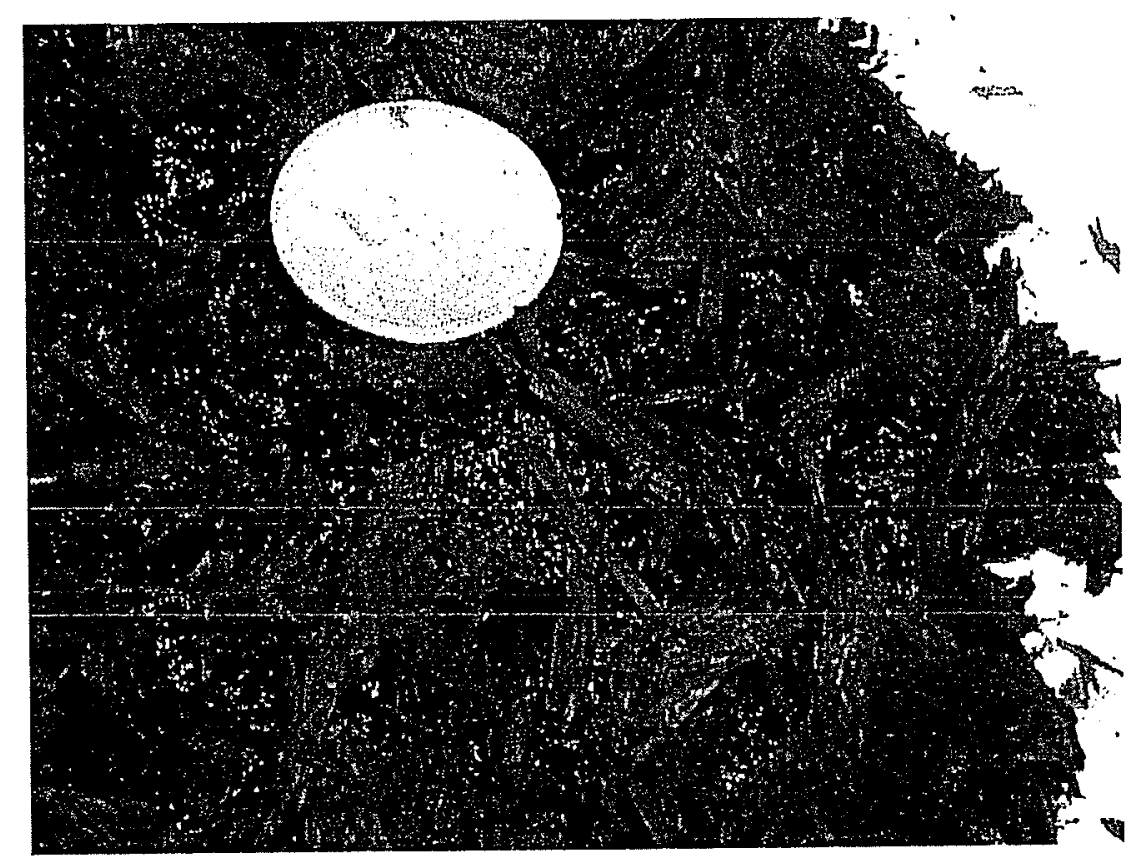

Figure A-10: Phase 1 composting, finished Recipe 2 (close-up). 


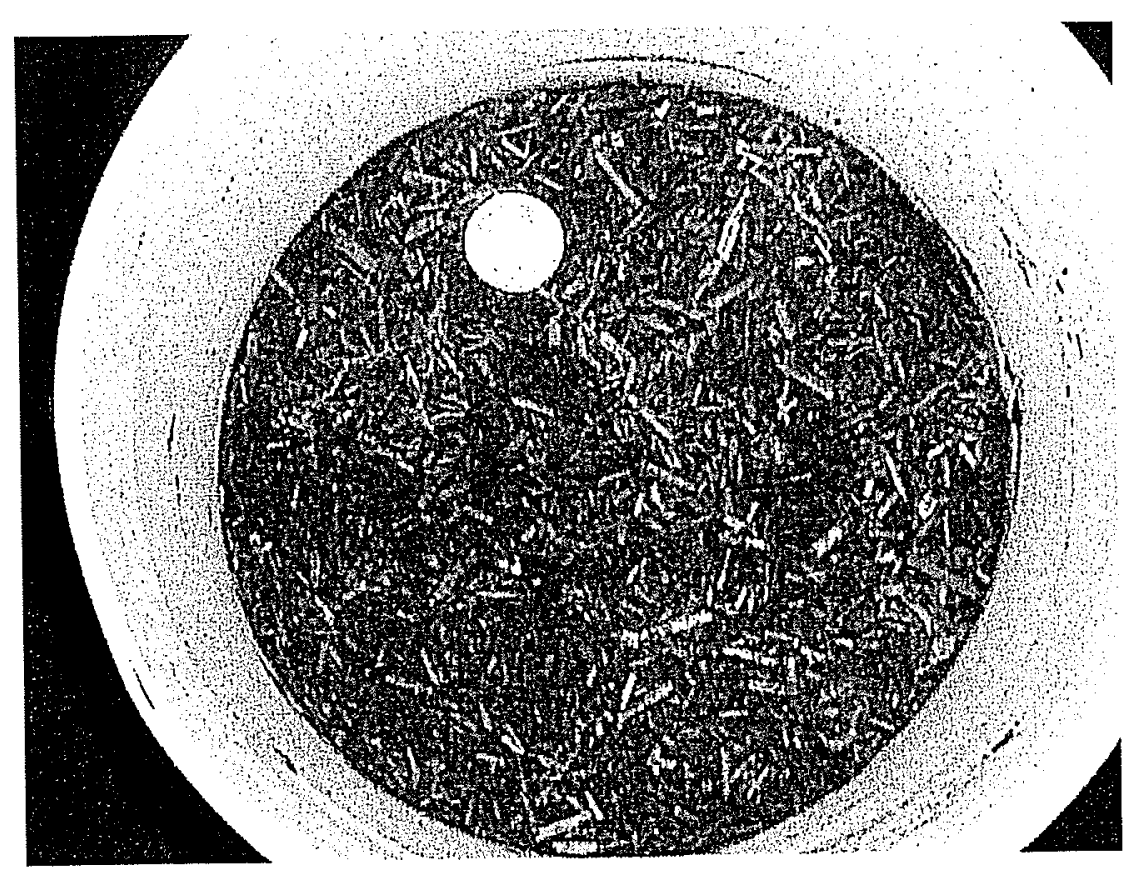

Figure A-11: Phase 1 composting, finished Recipe 3.

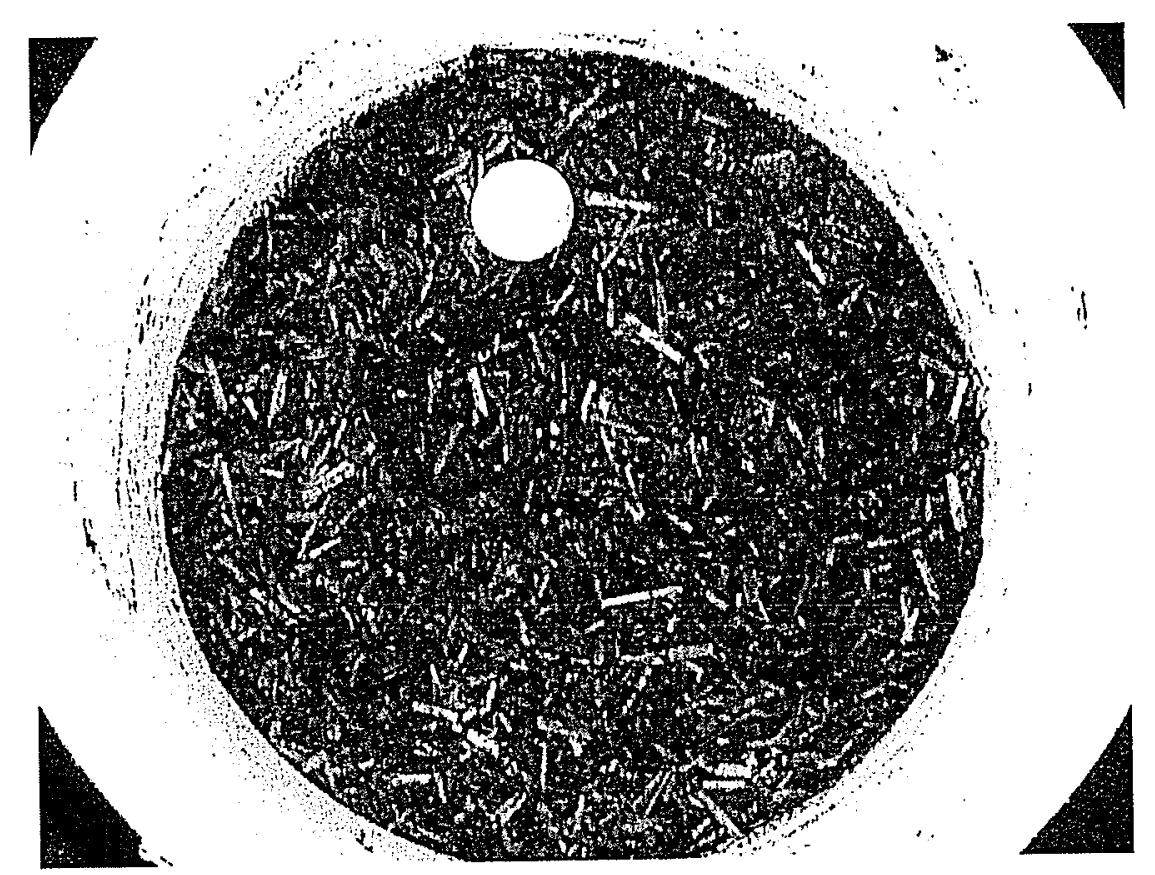

Figure A-12: Phase 1 composting, finished Recipe 4. 


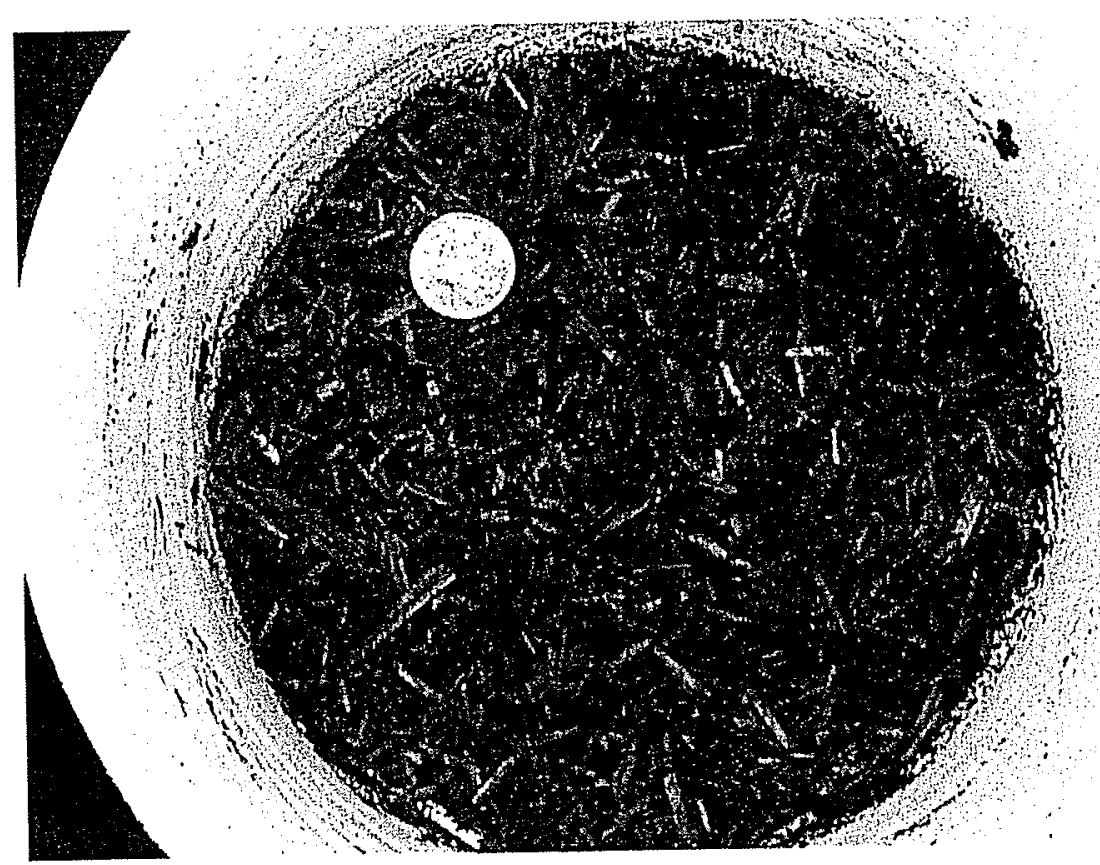

Figure A-13: Phase 1 composting, finished Recipe 5.

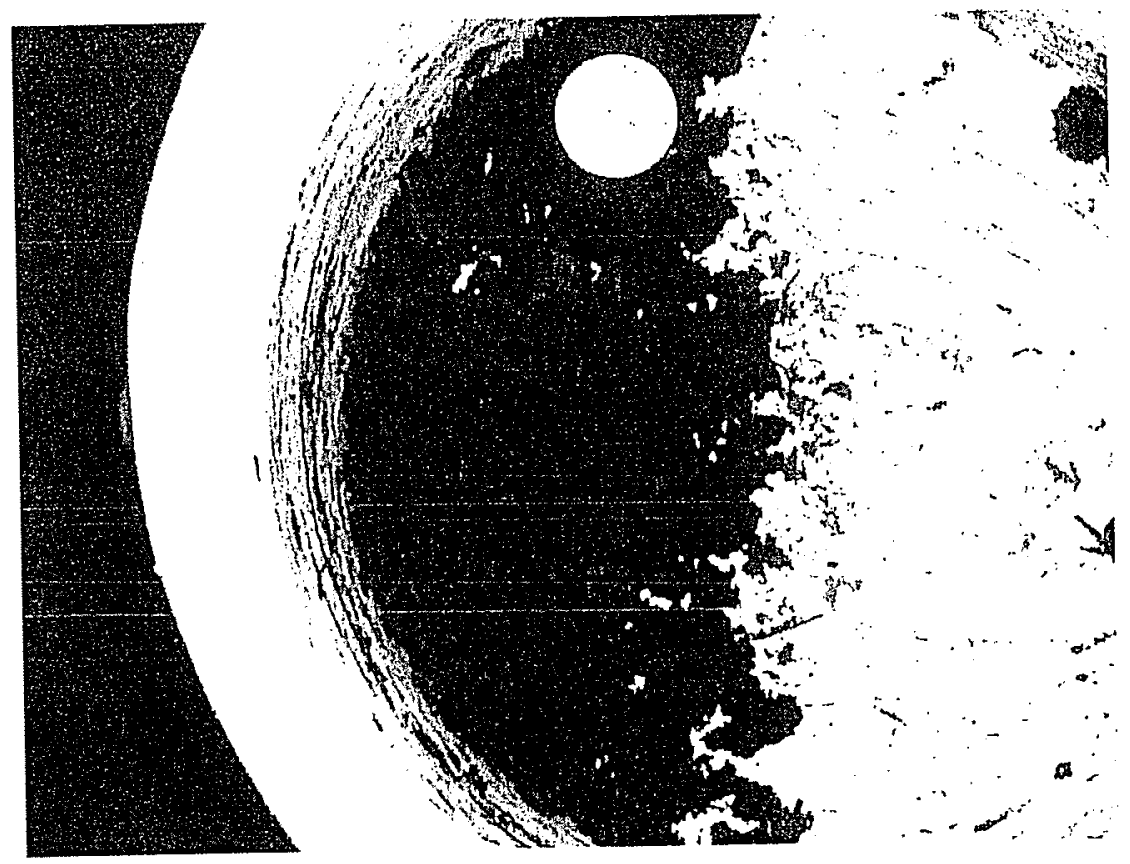

Figure A-14: Phase 1 composting, finished Recipe 6. 


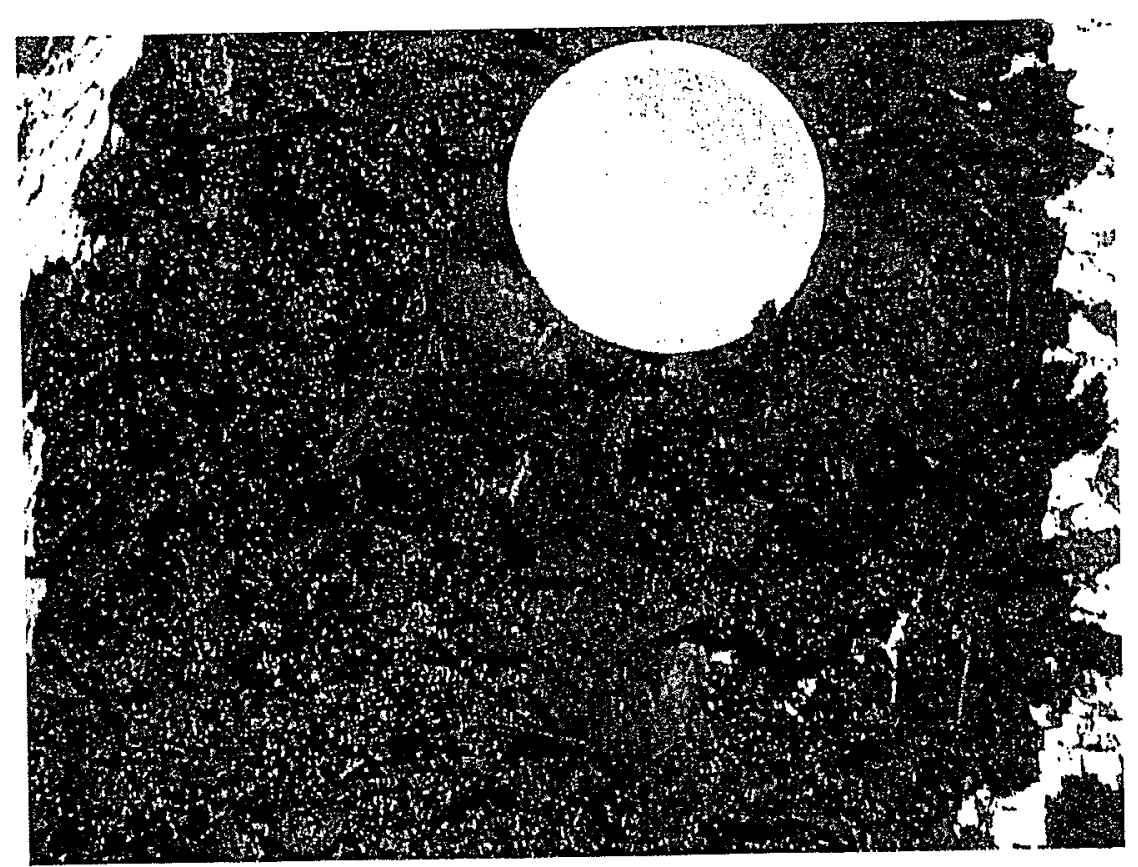

Figure A-15: Phase 1 composting, finished Recipe 6 (close-up).

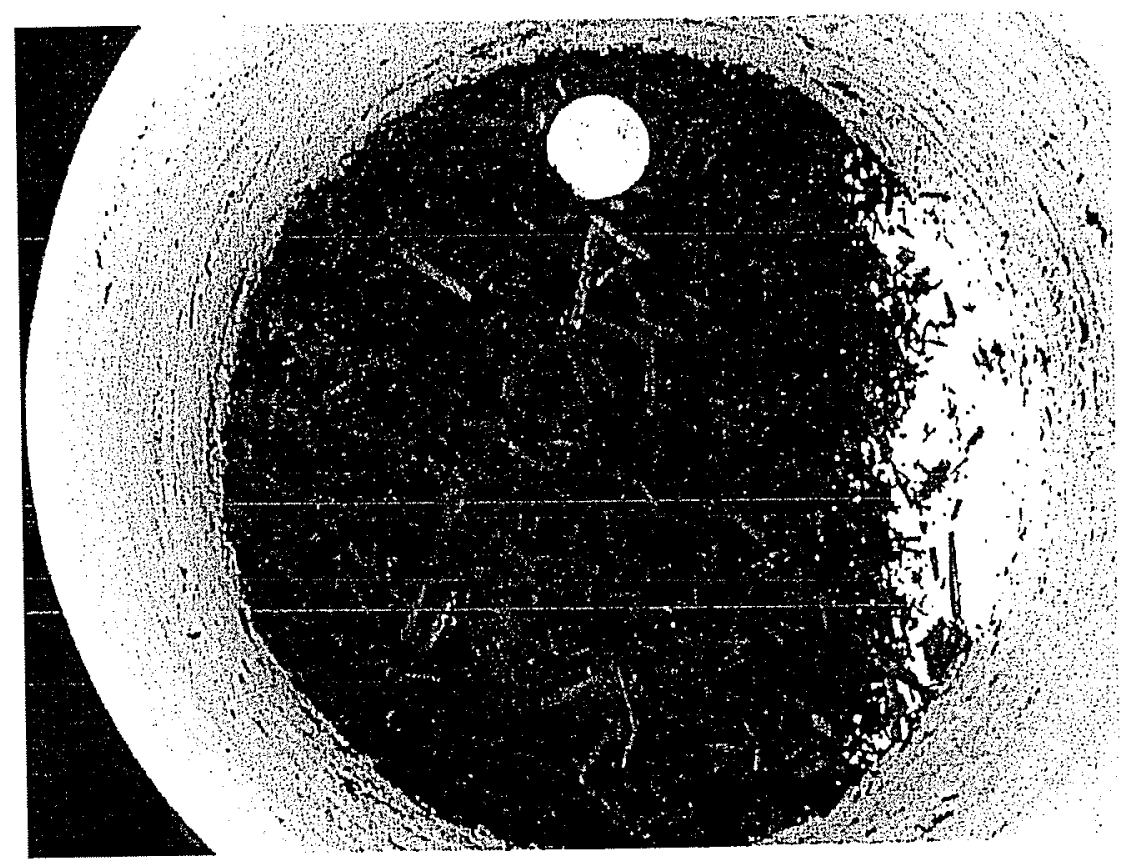

Figure A-16: Phase 1 composting, finished Recipe 7. 
APPENDIX B - PHASE 2 COMPOSTING PHOTOGRAPHS 


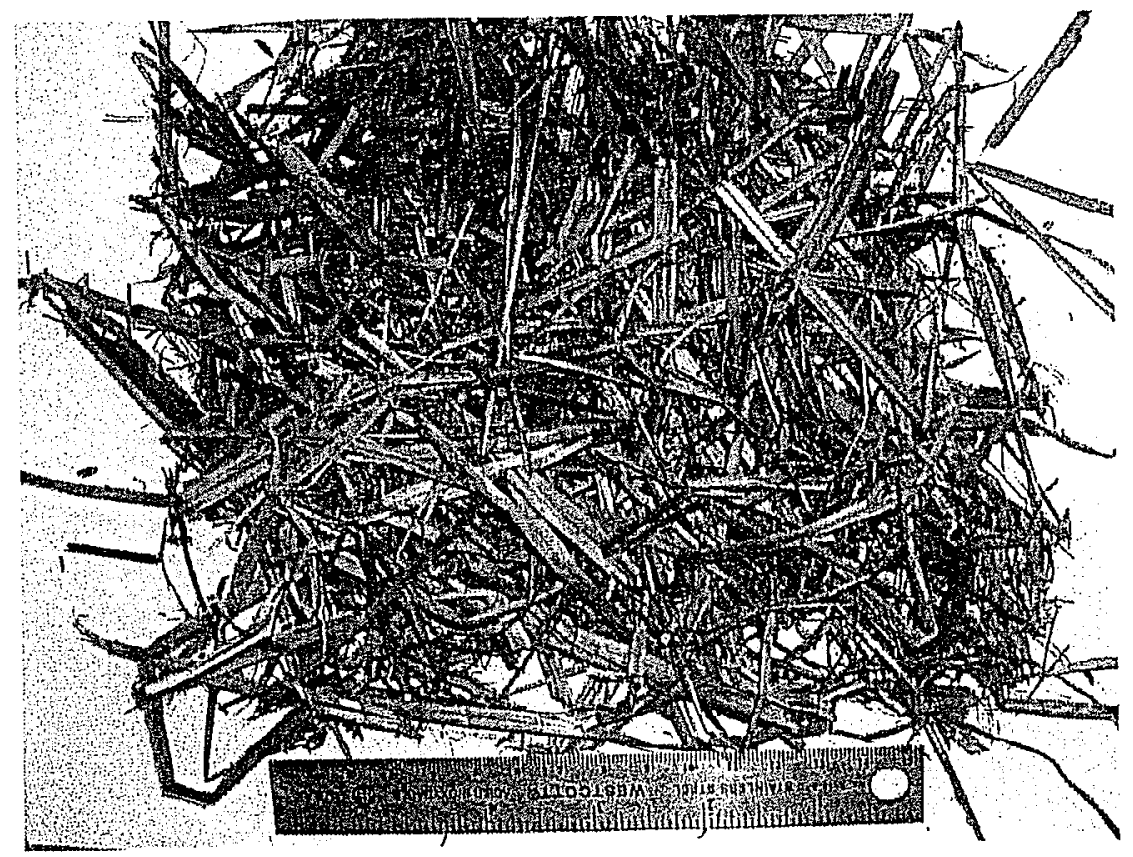

Figure B-1: Phase 2 composting, initial Recipe 1.

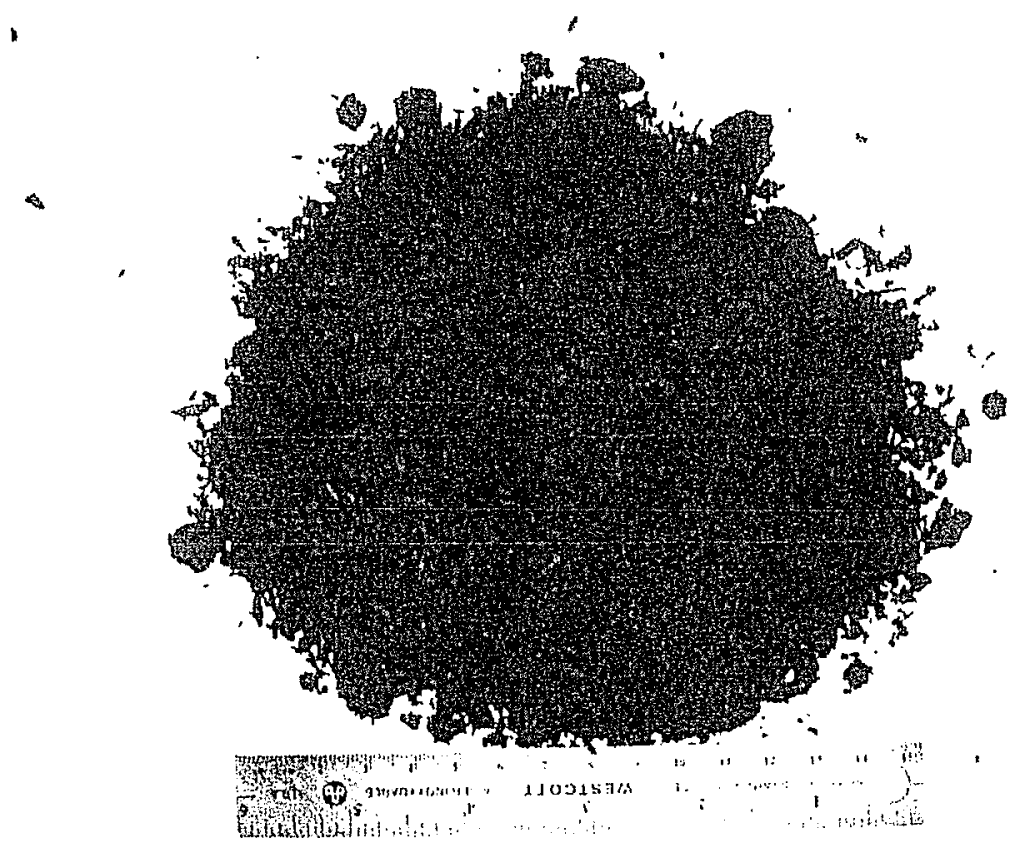

Figure B-2: Phase 2 composting, finished Recipe 1. 


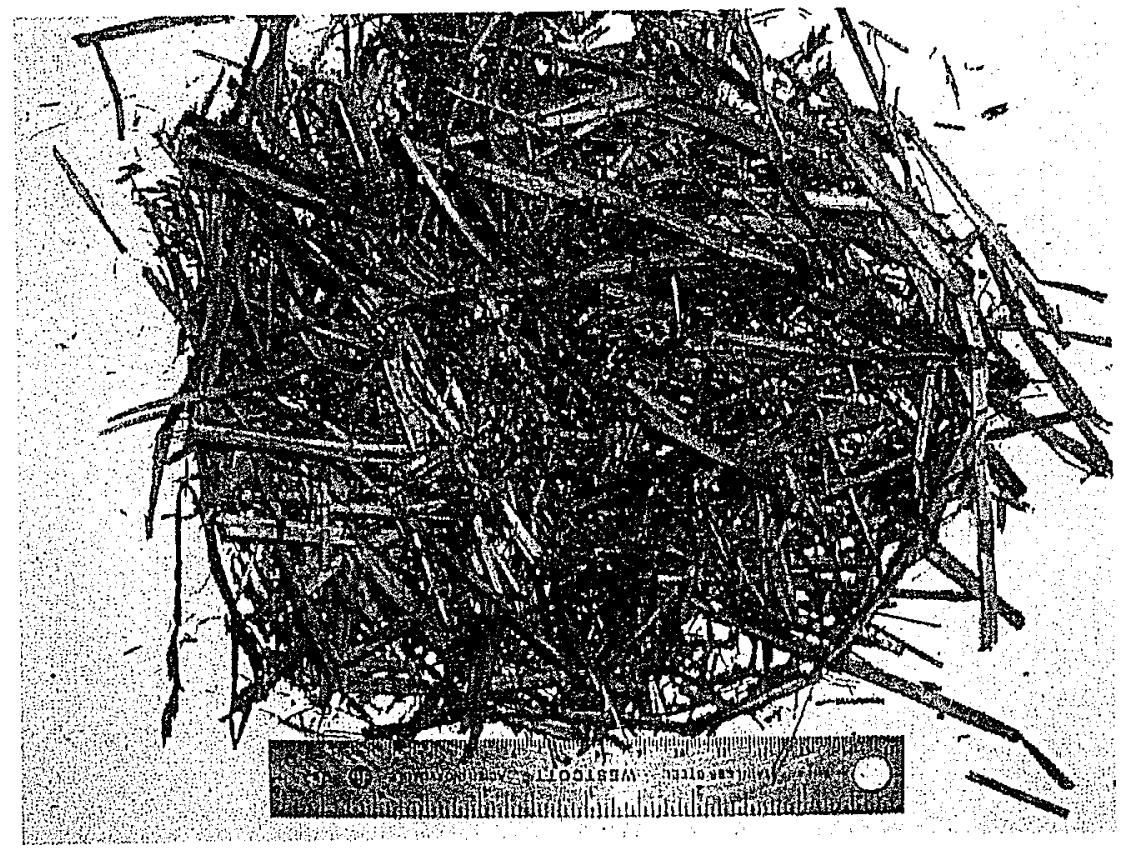

Figure B-3: Phase 2 composting, initial Recipe 2.

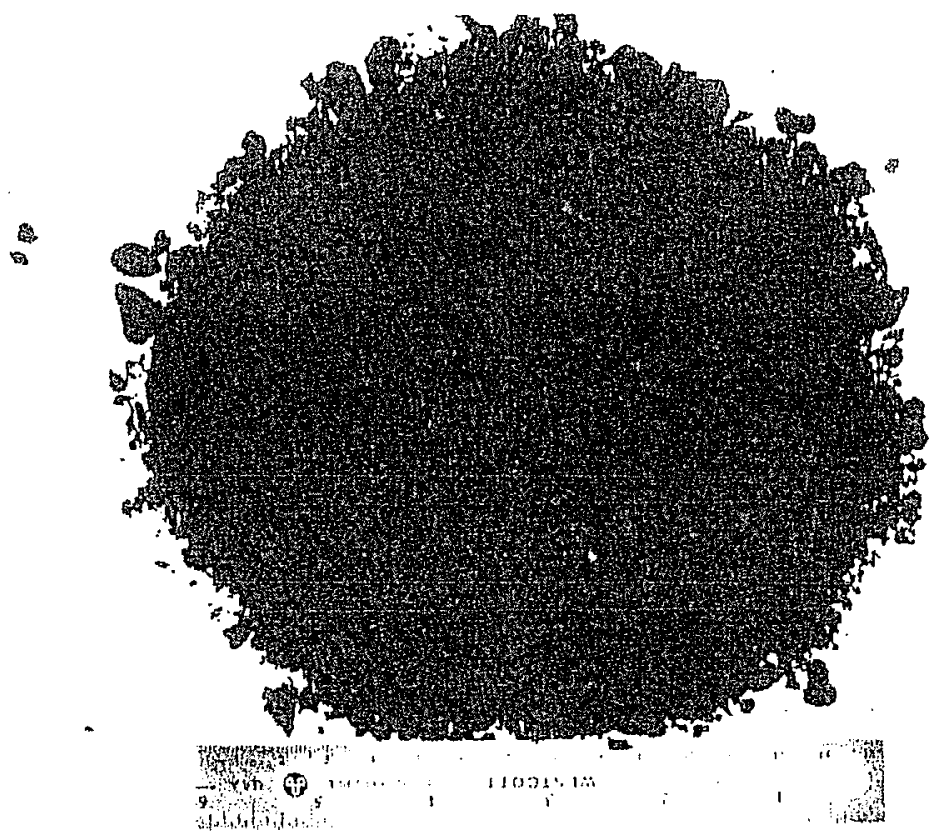

Figure B-4: Phase 2 composting, finished Recipe 2. 


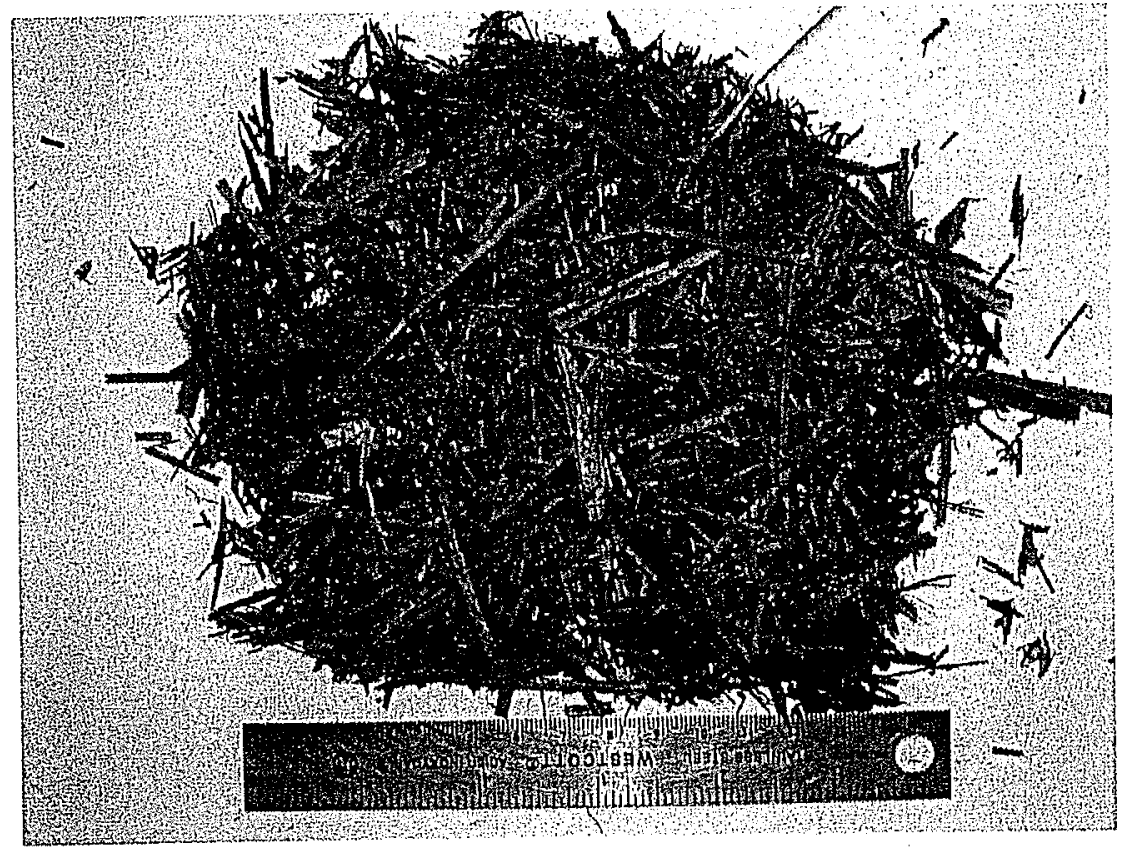

Figure B-5: Phase 2 composting, initial Recipe 3.

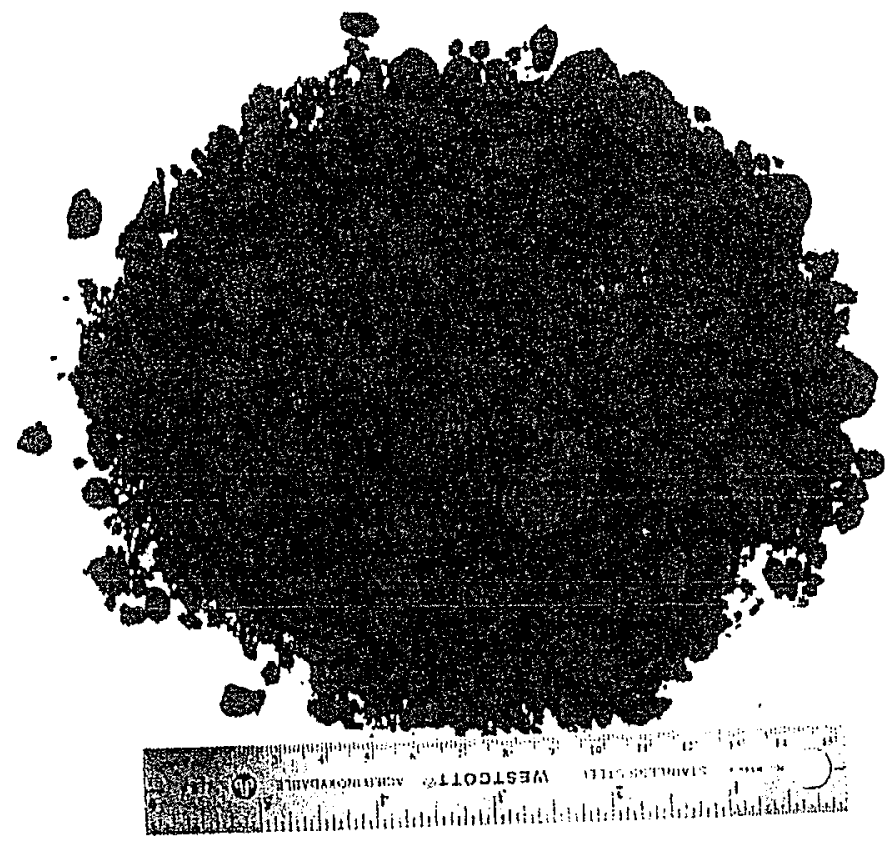

Figure B-6: Phase 2 composting, finished Recipe 3. 


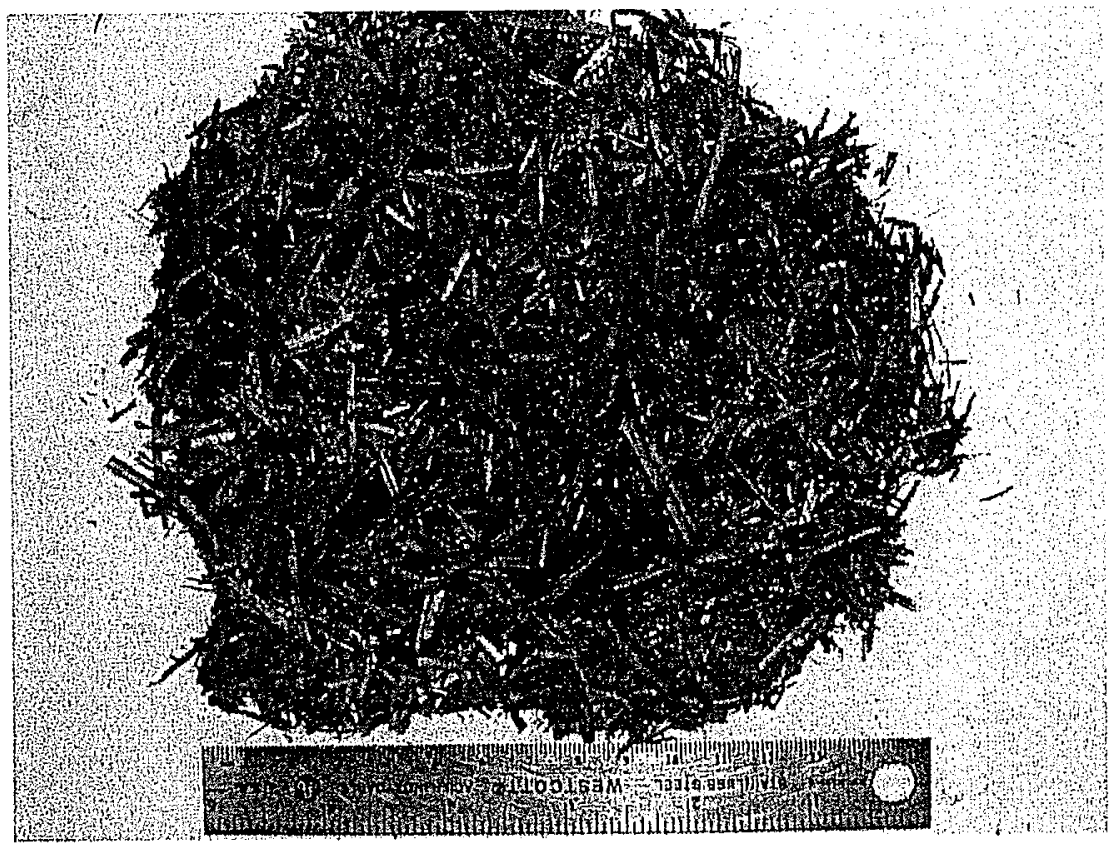

Figure B-7: Phase 2 composting, initial Recipe 4.

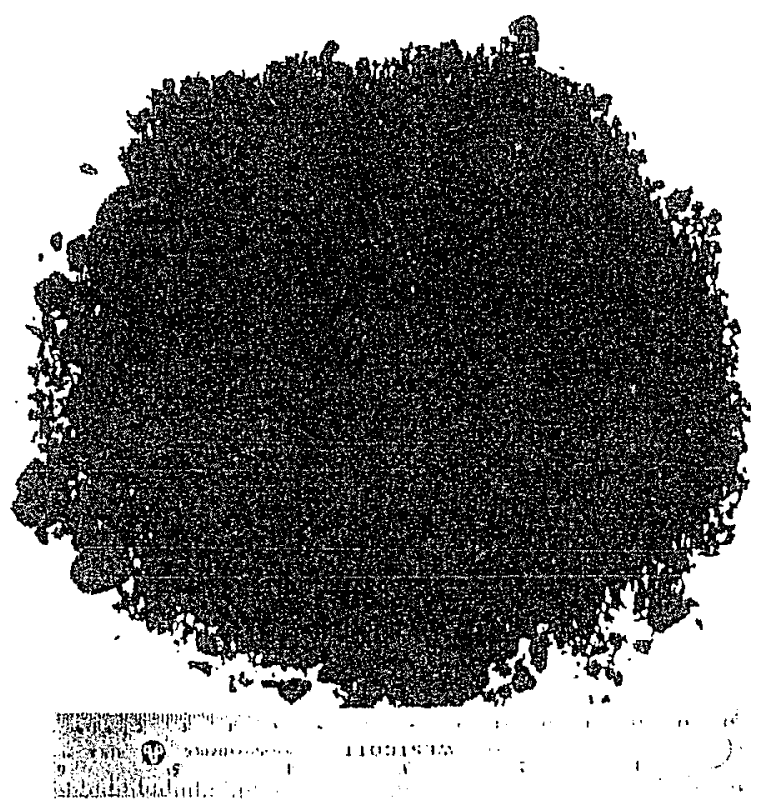

Figure B-8: Phase 2 composting, finished Recipe 4. 
APPENDIX C - PHASE 1 EXPERIMENTAL LABORATORY DATA 

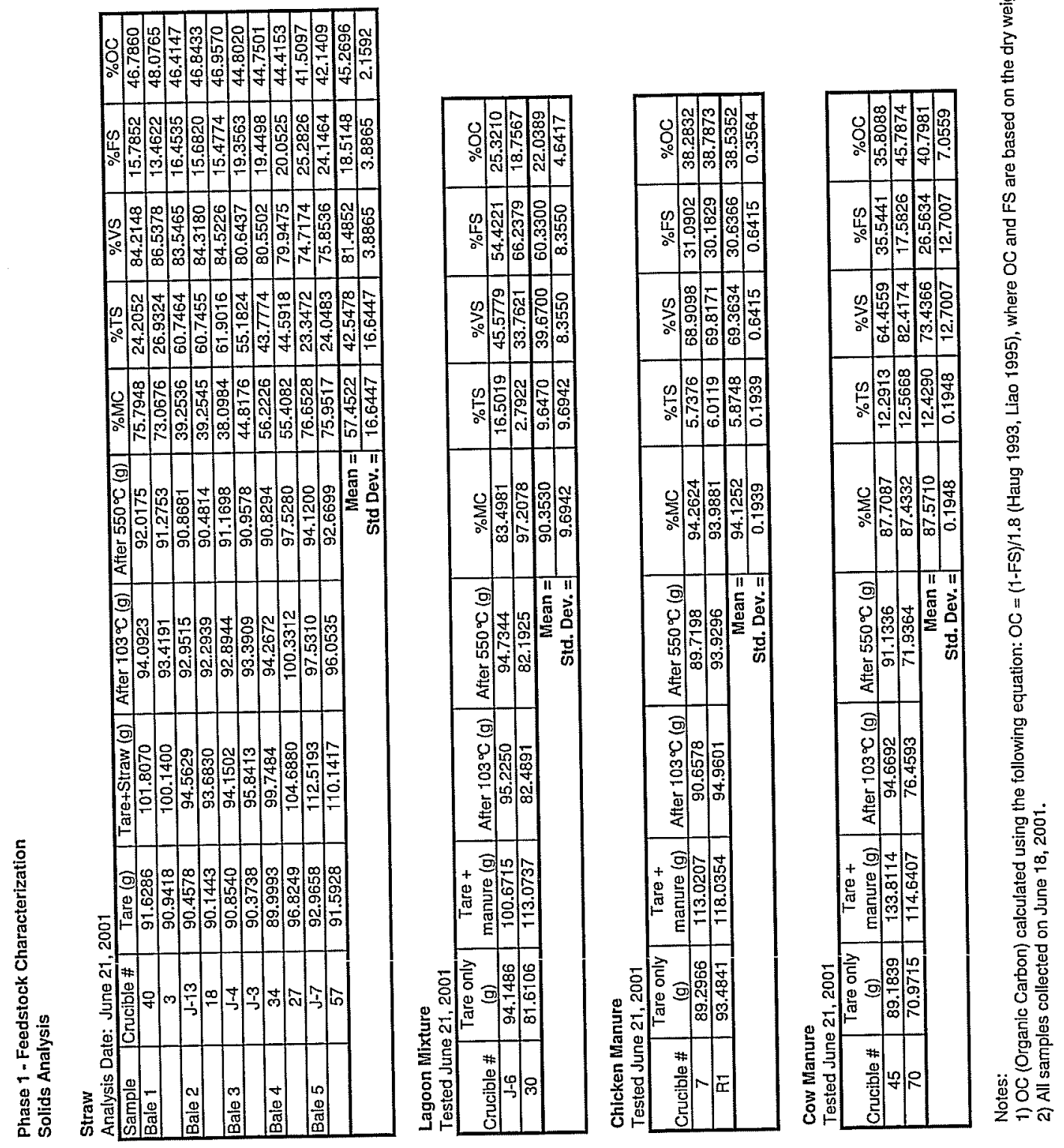


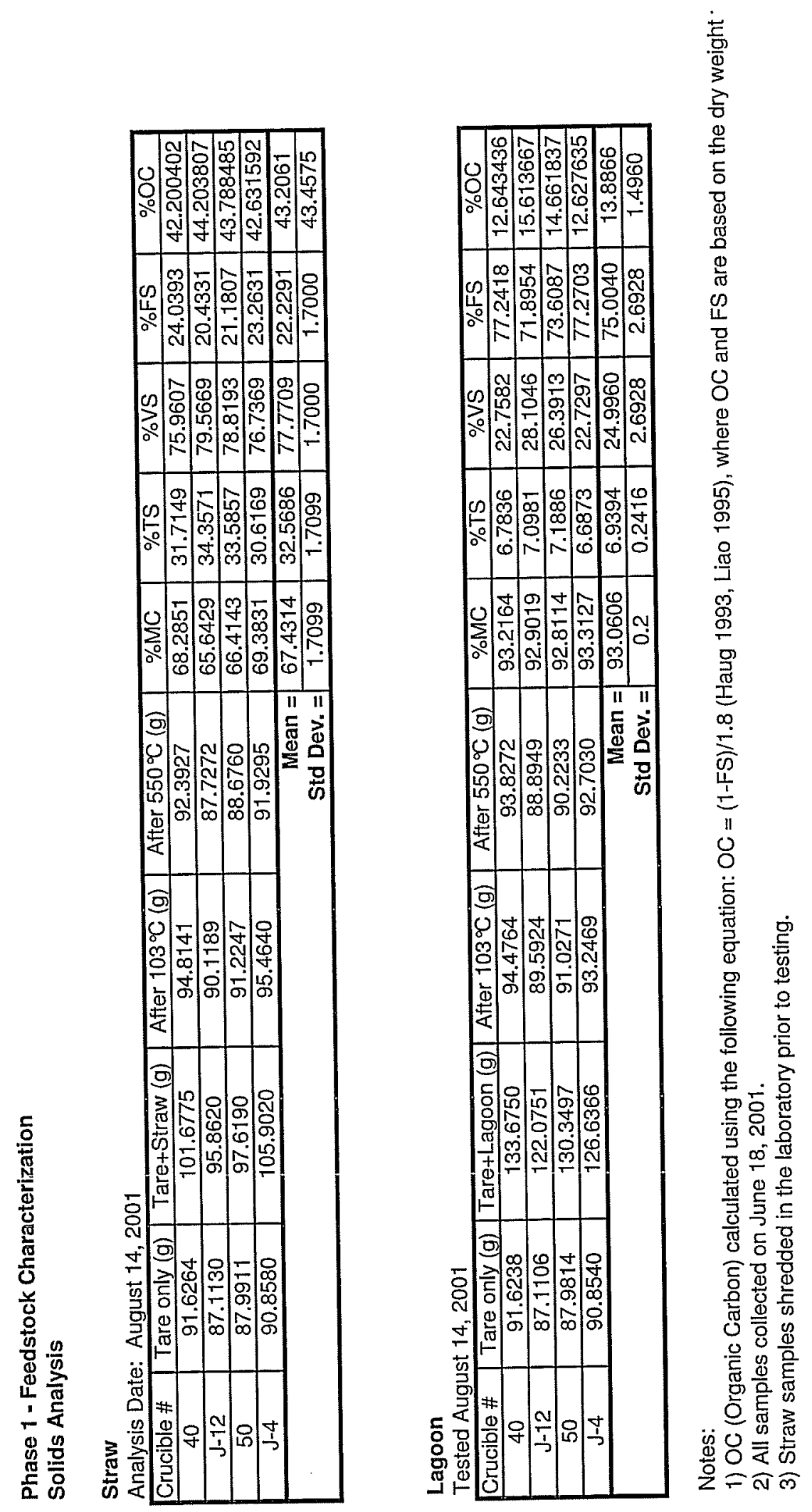


Test Date: August 23, 2001

$\% O C=(100-\% F S) / 1.8$

\begin{tabular}{|c|c|c|c|c|c|c|c|c|c|c|}
\hline Reactor & Crucible\# & Tare only (g) & Tare+Material $(\mathrm{g})$ & After $103^{\circ} \mathrm{C}(\mathrm{g})$ & After $550^{\circ} \mathrm{C}(\mathrm{g})$ & $\% M C$ & $\%$ TS & $\%$ VS & $\% F S$ & $\% O c(d b)$ \\
\hline \multirow[t]{3}{*}{$1 \mathrm{a}$} & 33 & 91.0635 & 100.4222 & 94.7081 & 91.7496 & 61.06 & 38.94 & 81.17 & 18.83 & \\
\hline & T9 & 89.7546 & 97.4908 & 93.0133 & 90.3179 & 57.88 & 42.12 & 82.71 & 17.29 & \\
\hline & T3 & 87.3404 & 96.2040 & 91.1645 & 87.9964 & 56.86 & 43.14 & 82.85 & 17.15 & \\
\hline \multicolumn{6}{|l|}{ 1a Mean } & 58.60 & 41.40 & 82.24 & 17.76 & 45.7 \\
\hline \multicolumn{6}{|l|}{ 1a St. Dev. } & 2.19 & 2.19 & 0.93 & 0.93 & 0.93 \\
\hline \multirow[t]{3}{*}{$1 \mathrm{~b}$} & 74 & 85.5421 & 94.3134 & 89.2228 & 86.2203 & 58.04 & 41.96 & 81.57 & 18.43 & \\
\hline & $\mathrm{n} n 35 \mathrm{~A}$ & 89.8343 & 98.0622 & 93.1754 & 90.4455 & 59.39 & 40.61 & 81.71 & 18.29 & \\
\hline & nn red & 86.0176 & 94.9306 & 89.8346 & 86.6636 & 57.17 & 42.83 & 83.08 & 16.92 & \\
\hline \multicolumn{6}{|l|}{$1 \mathrm{~b}$ Mean } & 58.20 & 41.80 & 82.12 & 17.88 & 45.6 \\
\hline \multirow{2}{*}{\multicolumn{6}{|c|}{$1 \mathrm{~b}$ St. Dev. }} & 1.12 & 1.12 & 0.83 & 0.83 & 0.83 \\
\hline \multirow{2}{*}{\multicolumn{8}{|c|}{$\begin{array}{l}\text { Recipe } 1 \text { Mean } \\
\text { Recipe } 1 \text { St. Dev. (between 1a and Ib) }\end{array}$}} & 82.18 & & 45.7 \\
\hline & & & & & & & & 0.09 & & 0.05 \\
\hline \multicolumn{11}{|c|}{ 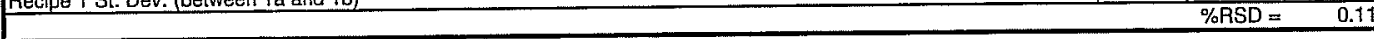 } \\
\hline
\end{tabular}

\begin{tabular}{|c|c|c|c|c|c|c|c|c|c|c|}
\hline Feactor & Crucible \# & Tare only $(g)$ & Tare+Material (g) & After $103^{\circ} \mathrm{C}(\mathrm{g})$ & After $550^{\circ} \mathrm{C}(\mathrm{g})$ & $\% \mathrm{MC}$ & $\%$ TS & $\%$ VS & $\% \mathrm{FS}$ & $\% O C(\mathrm{db})$ \\
\hline \multirow{3}{*}{$2 \mathrm{a}$} & $\mathrm{G}$ & 104.0151 & 113.3129 & 106.7091 & 104.5036 & 71.03 & 28.97 & 81.87 & 18.13 & \\
\hline & $\mathrm{J}-8$ & 91.9104 & 101.4841 & 94.1991 & 92.3376 & 76.09 & 23.91 & 81.33 & 18.67 & \\
\hline & 56 & 89.9814 & 99.6713 & 92.8725 & 90.6066 & 70.16 & 29.84 & 78.38 & 21.62 & \\
\hline \multicolumn{6}{|l|}{ 2a Mean } & 72.43 & 27.57 & 80.53 & 19.47 & 44.7 \\
\hline \multicolumn{6}{|l|}{ 2a St. Dev. } & 3.20 & 3.20 & 1.88 & 1.88 & 1.88 \\
\hline \multirow[t]{3}{*}{$2 b$} & $\mathrm{H} 1$ & 90.8014 & 100.0070 & 93.2644 & 91.3221 & 73.24 & 26.76 & 78.86 & 21.14 & \\
\hline & T5 & 88.6136 & 98.5895 & 91.0144 & 89.1095 & 75.93 & 24.07 & 79.34 & 20.66 & \\
\hline & no handle & 90.3086 & 99.8356 & broke & - & & & & & \\
\hline \multicolumn{6}{|c|}{$2 b$ Mean } & 74.59 & 25.41 & 79.10 & 20.90 & 43.9 \\
\hline & 1.90 & 1.90 & 0.34 & 0.34 & 0.34 \\
\hline \multicolumn{8}{|c|}{ Reclpe 2 Mean } & 79.81 & & 44.3 \\
\hline \multicolumn{8}{|c|}{ Recipe 2 St. Dev. (between 2a and 2b) } & 1.01 & & 0.56 \\
\hline
\end{tabular}

\begin{tabular}{|c|c|c|c|c|c|c|c|c|c|c|}
\hline Reactor & Crucible \# & Tare only $(\mathrm{g})$ & Tare+Material (g) & After $103^{\circ} \mathrm{C}(\mathrm{g})$ & After $550^{\circ} \mathrm{C}(\mathrm{g})$ & $\% \mathrm{MC}$ & \%TS & $\%$ VS & $\% F S$ & $\% \mathrm{OC}(\mathrm{db})$ \\
\hline \multirow[t]{3}{*}{$3 a$} & $J-6$ & 81.6155 & 91.3352 & 87.2955 & 82.7220 & 41.56 & 58.44 & 80.52 & 19.48 & \\
\hline & 7 & 89.3041 & 99.1300 & 94.9324 & disturbed & 42.72 & 57.28 & & & \\
\hline & 3 & 90.9437 & 100.7719 & 96.6654 & 92.0090 & 41.78 & 58.22 & 81.38 & 18.62 & \\
\hline \multicolumn{6}{|l|}{ 3a Mean } & 42.02 & 57.98 & 80.95 & 19.05 & 45.0 \\
\hline \multicolumn{6}{|l|}{ 3a St. Dev. } & 0.61 & 0.61 & 0.61 & 0.61 & 0.61 \\
\hline \multirow[t]{3}{*}{$3 \mathrm{~b}$} & R1 & 93.4913 & 103.2218 & 99.0485 & 94.6246 & 42.89 & 57.11 & 79.61 & 20.39 & \\
\hline & 2 & 91.8600 & 100.3108 & 96.7478 & 92.7934 & 42.16 & 57.84 & 80.90 & 19.10 & \\
\hline & 25 & 92.1652 & 99.9084 & 96.7553 & 93.0503 & 40.72 & 59.28 & 80.72 & 19.28 & \\
\hline \multicolumn{6}{|c|}{$3 b$ Mean } & 41.92 & 58.08 & 80.41 & 19.59 & 44.7 \\
\hline & 1.10 & 1.10 & 0.70 & 0.70 & 0.70 \\
\hline \multicolumn{8}{|c|}{ Recipe 3 Mean } & 80.68 & & 44.8 \\
\hline \multicolumn{8}{|c|}{ Recipe 3 St. Dev. (between 3a and 3b) } & 0.38 & & 0.21 \\
\hline & & & & & & & & & $\%$ RSD $=$ & 0.47 \\
\hline
\end{tabular}

\begin{tabular}{|c|c|c|c|c|c|c|c|c|c|c|}
\hline Reactor & Crucible \# & Tare only $(\mathrm{g})$ & Tare+Material (g) & After $103^{\circ} \mathrm{C}(\mathrm{g})$ & After $550^{\circ} \mathrm{C}(\mathrm{g})$ & $\% \mathrm{MC}$ & $\%$ TS & \%VS & $\% \mathrm{FS}$ & $\% 0 \mathrm{c}(\mathrm{db})$ \\
\hline \multirow[t]{3}{*}{$4 \mathrm{a}$} & $A-7$ & 91.3107 & 100.9540 & 95.0450 & 92.0798 & 61.28 & 38.72 & 79.40 & 20.60 & \\
\hline & 49 & 90.9486 & 99.2875 & 94.3628 & 91.6143 & 59.06 & 40.94 & 80.50 & 19.50 & \\
\hline & A-11 & 89.1607 & 98.8374 & 93.6262 & 90.0488 & 53.85 & 46.15 & 80.11 & 19.89 & \\
\hline \multicolumn{6}{|l|}{ 4a Mean } & 58.06 & 41.94 & 80.01 & 19.99 & 44.4 \\
\hline \multicolumn{6}{|l|}{ 4a St. Dev. } & 3.81 & 3.81 & 0.56 & 0.56 & 0.56 \\
\hline \multirow[t]{3}{*}{$4 \mathrm{~b}$} & $A-8$ & 89.8055 & 98.5957 & 92.0875 & 90.2541 & 74.04 & 25.96 & 80.34 & 19.66 & \\
\hline & T8 & 88.0321 & 97.7744 & 91.8040 & 88.7635 & 61.28 & 38.72 & 80.61 & 19.39 & \\
\hline & 45 & 89.1923 & 98.5330 & 93.3620 & 90.0294 & 55.36 & 44.64 & 79.92 & 20.08 & \\
\hline \multicolumn{6}{|l|}{ 4b Mean } & 63.56 & 36.44 & 80.29 & 19.71 & 44.6 \\
\hline \multicolumn{6}{|c|}{ 4b St. Dev. } & 9.55 & 9.55 & 0.35 & 0.35 & 0.35 \\
\hline \multicolumn{8}{|c|}{ Recipe 4 Mean } & 80.15 & & 44.5 \\
\hline \multicolumn{8}{|c|}{ Recipe 4 St. Dev. (between 4a and 4b) } & 0.20 & & 0.11 \\
\hline
\end{tabular}




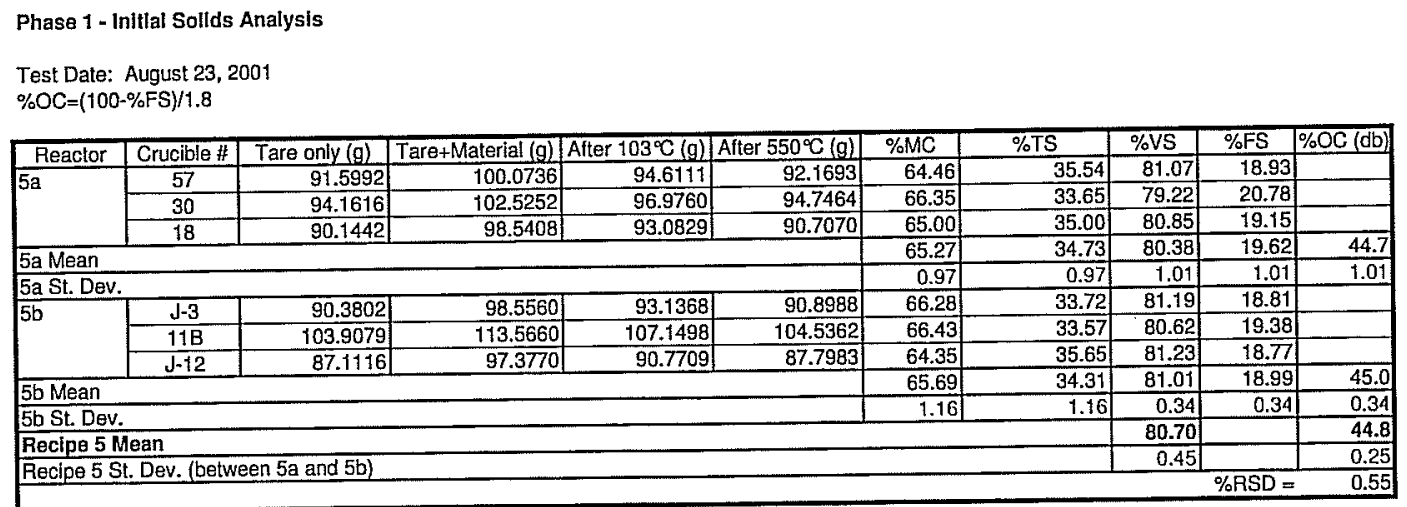

\begin{tabular}{|c|c|c|c|c|c|c|c|c|c|c|}
\hline Reactor & Crucible \# & Tare only $(g)$ & Tare+Material (g) & After $103^{\circ} \mathrm{C}(\mathrm{g})$ & After $550^{\circ} \mathrm{C}(\mathrm{g})$ & $\% \mathrm{MC}$ & $\% \mathrm{TS}$ & $\%$ VS & $\%$ FS & $\% \mathrm{OC}(\mathrm{db})$ \\
\hline \multirow[t]{3}{*}{$6 \mathrm{a}$} & 70 & 71.0105 & 80.1151 & 73.4060 & \begin{tabular}{|l|}
71.4079 \\
\end{tabular} & 73.69 & 26.31 & 83.41 & 16.59 & \\
\hline & T2 & 88.1434 & 97.1422 & 90.6664 & 88.6111 & 71.96 & 28.04 & 81.46 & 18.54 & \\
\hline & $\operatorname{nn} 37-P$ & 71.6303 & 80.3980 & 74.0238 & 72.1103 & 72.70 & 27.30 & 79.95 & 20.05 & \\
\hline \multicolumn{6}{|l|}{ 6a Mean } & 72.78 & 27.22 & 81.61 & 18.39 & 45.3 \\
\hline \multicolumn{6}{|l|}{ 6a St. Dev. } & 0.87 & 0.87 & 1.74 & 1.74 & 1.74 \\
\hline \multirow[t]{3}{*}{$6 \mathrm{~b}$} & $\mathrm{~T} 7$ & 88.6406 & 97.8214 & 91.2320 & 89.1261 & 71.77 & 28.23 & 81.26 & 18.74 & \\
\hline & 32 & 94.0833 & 104.0430 & 96.8098 & 94.6156 & 72.62 & 27.38 & 80.48 & 19.52 & \\
\hline & 50 & 90.7168 & 99.6220 & 93.0990 & 91.1848 & 73.25 & 26.75 & 80.35 & 19.65 & \\
\hline \multicolumn{6}{|c|}{$6 b$ Mean } & 72.55 & 27.45 & 80.70 & 19.30 & 44.8 \\
\hline \multirow{2}{*}{\multicolumn{6}{|c|}{$\begin{array}{l}\text { 6b St. Dev. } \\
\text { Reclpe } 6 \text { Mean }\end{array}$}} & 0.74 & 0.74 & 0.49 & 0.49 & 0.49 \\
\hline & \multirow{2}{*}{\multicolumn{7}{|c|}{ Reclpe 6 Mean }} & 81.15 & & 45.1 \\
\hline \multicolumn{5}{|c|}{ Recipe 6 St. Dev. (between 6a and 6b) } & & & & 0.64 & & 0.36 \\
\hline
\end{tabular}

\begin{tabular}{|c|c|c|c|c|c|c|c|c|c|c|}
\hline Reactor & Crucible \# & Tare only $(\mathrm{g})$ & Tare+Material $(g)$ & After $103^{\circ} \mathrm{C}(\mathrm{g})$ & Aiter $550^{\circ} \mathrm{C}(\mathrm{g})$ & $\% M C$ & \%TS & $\%$ VS & $\% \mathrm{FS}$ & $\% \circ \mathrm{O}(\mathrm{db})$ \\
\hline \multirow[t]{3}{*}{$7 a$} & $\mathrm{~J}-4$ & 90.8567 & 98.7456 & \begin{tabular}{|r|}
93.7624 \\
\end{tabular} & 91.4017 & 63.17 & 36.83 & 81.24 & 18.76 & \\
\hline & 50 & 87.9836 & 96.2455 & 90.9268 & 88.5664 & 64.38 & 35.62 & 80.20 & 19.80 & \\
\hline & 40 & 91.6251 & 99.8118 & 94.4188 & 92.1948 & 65.88 & 34.12 & 79.61 & 20.39 & \\
\hline \multicolumn{6}{|l|}{7 a Mean } & 64.47 & 35.53 & 80.35 & 19.65 & 44.6 \\
\hline \multicolumn{6}{|l|}{ 7a St. Dev. } & 1.36 & 1.36 & 0.83 & 0.83 & 0.83 \\
\hline \multirow[t]{3}{*}{$7 \mathrm{~b}$} & 34 & 90.0013 & 99.2850 & 93.4540 & 90.6333 & 62.81 & 37.19 & 81.70 & 18.30 & \\
\hline & $\mathrm{J}-7$ & 92.9659 & 102.8898 & 96.1604 & 93.6449 & 67.81 & 32.19 & 78.74 & 21.26 & \\
\hline & TI & 87.7708 & 95.9265 & 90.4678 & 88.2976 & 66.93 & 33.07 & 80.47 & 19.53 & \\
\hline \multicolumn{6}{|l|}{$7 \mathrm{~b}$ Mean } & 65.85 & 34.15 & 80.30 & 19.70 & 44.6 \\
\hline \multicolumn{6}{|c|}{ 7b St. Dev. } & 2.67 & 2.67 & 1.48 & 1.48 & 1.48 \\
\hline \multirow{2}{*}{\multicolumn{8}{|c|}{ Recipe 7 Mean }} & 80.33 & & 44.6 \\
\hline \multicolumn{7}{|c|}{ Recipe 7 Mean } & & 0.03 & & 0.02 \\
\hline
\end{tabular}




\section{Phase 1 - Finat Solids Analysis}

Tested October 31, 2001

$\% O C=(100-\% F S) / 1.8$

\begin{tabular}{|c|c|c|c|c|c|c|c|c|c|c|}
\hline Peactor & Crucible \# & Tare only (g) & Tare+Material (g) & After $103^{\circ} \mathrm{C}(\mathrm{g})$ & After $550^{\circ} \mathrm{C}(\mathrm{g})$ & $\% M C$ & \%TS & $\%$ VS & $\%$ FS & $\% \mathrm{OC}(\mathrm{db})$ \\
\hline \multirow[t]{3}{*}{$1 \mathrm{a}$} & 56 & 89.9819 & 98.0829 & 93.8353 & 90.9853 & 52.43 & 47.57 & 73.96 & 26.04 & \\
\hline & $\mathrm{J}-8$ & 91.9117 & 102.5341 & 96.9910 & 93.2748 & 52.18 & 47.82 & 73.16 & 26,84 & \\
\hline & 57 & 91.5969 & 101.7965 & 96.5091 & 92.9050 & 51.84 & 48.16 & 73.37 & 26.63 & \\
\hline \multicolumn{6}{|l|}{ ta Mean } & 52.15 & 47.85 & 73.50 & 26.50 & 40.8 \\
\hline \multicolumn{6}{|c|}{ Ia St. Dev. } & 0.30 & 0.30 & 0.41 & 0.41 & 0.41 \\
\hline \multirow[t]{3}{*}{$\mathrm{ab}$} & 74 & 85.5431 & 94.9780 & 90.0149 & 86.7520 & 52.60 & 47.40 & 72.97 & 27.03 & \\
\hline & $J-4$ & 90.8591 & 101.5570 & 95.8571 & 92.1659 & 53.28 & 46.72 & 73.85 & 26.15 & \\
\hline & 3 & 90.9443 & 100.3640 & 95.4240 & 92.1560 & 52.44 & 47.56 & 72.95 & 27.05 & \\
\hline \multicolumn{6}{|c|}{ 16 Mean } & 52.78 & 47.22 & 73.26 & 26.74 & 40.7 \\
\hline \multicolumn{6}{|c|}{ 1b St. Dev. } & 0.44 & 0.44 & 0.52 & 0.52 & 0.52 \\
\hline \multicolumn{8}{|c|}{ Recipe 1 Mean } & 73.38 & & 40.8 \\
\hline \multicolumn{8}{|c|}{ Recipe 1 St. Dev. (between ta and tb) } & 0.17 & & 0.09 \\
\hline
\end{tabular}

\begin{tabular}{|c|c|c|c|c|c|c|c|c|c|c|}
\hline Reactor & Grucible \# & Tare only $(g)$ & Tare+Material $(\mathrm{g})$ & After $103^{\circ} \mathrm{C}(\mathrm{g})$ & After $550^{\circ} \mathrm{C}(\mathrm{g})$ & $\% \mathrm{MC}$ & $\% \mathrm{TS}$ & $\%$ VS & $\%$ FS & $\% O C$ (db) \\
\hline \multirow[t]{3}{*}{$2 \mathrm{a}$} & $T 8$ & 88.0349 & 105.2603 & 93.3057 & 89.8116 & 69.40 & 30.60 & 66.29 & 33.71 & \\
\hline & $\bar{A}-11$ & 89.1616 & 107.1790 & 94.7530 & 91.0706 & 68.97 & 31.03 & 65.86 & 34.14 & \\
\hline & 45 & 89.1936 & 105.6909 & 94.1988 & 90.8532 & 69.66 & 30.34 & 66.84 & 33.16 & \\
\hline \multicolumn{6}{|c|}{ 2a Mean } & 69.34 & 30.66 & 66.33 & 33.67 & 36.9 \\
\hline \multicolumn{6}{|c|}{ 2a St. Dev. } & 0.35 & 0.35 & 0.49 & 0.49 & 0.49 \\
\hline \multirow[t]{3}{*}{$2 b$} & 7 & 89.3068 & 105.2142 & 93.9465 & 90.9579 & 70.83 & 29.17 & 64.41 & 35.59 & \\
\hline & 30 & 94.1564 & 112.3161 & 99.3728 & 96.0314 & 71.27 & 28.73 & 64.06 & 35.94 & \\
\hline & $\sqrt{-6}$ & 81.6164 & 98.0611 & 86.4302 & 83.3730 & 70.73 & 29.27 & 63.51 & 36.49 & \\
\hline \multicolumn{6}{|c|}{$2 \mathrm{~b}$ Mean } & 71.05 & 28.95 & 64.23 & 35.77 & 35.7 \\
\hline \multirow{2}{*}{\multicolumn{8}{|c|}{ Reclpe 2 Mean }} & 0.25 & 0.25 & 0.25 \\
\hline & & & & & & & & 65.28 & & 36.3 \\
\hline \multicolumn{8}{|c|}{ Recipe 2 St. Dev. (between 2a and 2b) } & 1.48 & & 0.82 \\
\hline
\end{tabular}

Recipe 2 St. Dev. (between 2a and 2b

\begin{tabular}{|c|c|c|c|c|c|c|c|c|c|c|}
\hline Reactor & Crucible\# & Tare only $(\mathrm{g})$ & Tare+Material (g) & After $103^{\circ} \mathrm{C}(\mathrm{g})$ & After $550^{\circ} \mathrm{C}(\mathrm{g})$ & $\% \mathrm{MC}$ & $\%$ TS & \%VS & $\%$ FS & $\% \mathrm{OG}(\mathrm{db})$ \\
\hline \multirow[t]{3}{*}{$3 a$} & $T 7$ & 88.6357 & $\begin{array}{r}98.7401 \\
\end{array}$ & 94.7339 & 90.4561 & 39.65 & 60.35 & 70.15 & 29.85 & \\
\hline & 33 & 91.0620 & 101.8165 & 97.5654 & cracked & 39.53 & 60.47 & & & \\
\hline & 40 & 91.6273 & 102.0515 & 98.0312 & 93.5516 & 38.57 & 61.43 & 69.95 & 30.05 & \\
\hline \multicolumn{6}{|c|}{ 3a Mean } & 39.25 & 60.75 & 70.05 & 29.95 & 38.9 \\
\hline \multicolumn{6}{|c|}{ 3a St. Dev. } & 0.59 & 0.59 & 0.14 & 0.14 & 0.14 \\
\hline \multirow[t]{3}{*}{$3 \mathrm{~b}$} & $\overline{\mathrm{R} 1}$ & 93.4911 & 102.4266 & 99.2175 & 95.2219 & 35.91 & 64.09 & 69.78 & 30.22 & \\
\hline & $G$ & 104.0152 & 111.8290 & 108.9163 & 105.4827 & 37.28 & 62.72 & 70.06 & 29.94 & \\
\hline & $\mathrm{d}-12$ & 87.1134 & 94.4370 & 91.5849 & 88.4296 & 38.94 & 61.06 & 70.56 & 29.44 & \\
\hline \multicolumn{6}{|c|}{$3 b$ Mean } & 37.38 & 62.62 & 70.13 & 29.87 & 39.0 \\
\hline \multicolumn{6}{|c|}{ 3b St. Dev. } & 1.52 & 1.52 & 0.40 & 0.40 & 0.40 \\
\hline Recipe 3 & & & & & & & & 70.09 & & 38.9 \\
\hline \multicolumn{8}{|c|}{$\begin{array}{l}\text { Recipe } 3 \text { Mean } \\
\text { Recipe } 3 \text { St. Dev. (between } 3 a \text { and } 3 b \text { ) }\end{array}$} & 0.06 & & 0.03 \\
\hline Reactor & Crucible \# & Tare only (g) & Tare+Material (g) & After $103^{\circ} \mathrm{C}(\mathrm{g})$ & After $550^{\circ} \mathrm{C}(\mathrm{g})$ & $\% M C$ & $\%$ TS & $\%$ VS & $\% F S$ & $\% O C$ (db) \\
\hline \multirow[t]{3}{*}{$4 a$} & $\mathrm{~T} 1$ & 87.7720 & 97.6890 & 92.6022 & 89.5120 & 51.29 & 48.71 & 63.98 & 36.02 & \\
\hline & 32 & 94.0810 & 105.3106 & 99.5265 & 96.0453 & 51.51 & 48.49 & 63.93 & 36.07 & \\
\hline & T5 & 88.6123 & 99.6614 & 93.9659 & 90.5314 & 51.55 & 48.45 & 64.15 & 35.85 & \\
\hline \multicolumn{6}{|c|}{ 4a Mean } & 51.45 & 48.55 & 64.02 & 35.98 & 35.6 \\
\hline \multicolumn{6}{|c|}{ 4a St. Dev. } & 0.14 & 0.14 & 0.12 & 0.12 & 0.12 \\
\hline \multirow[t]{3}{*}{$4 \mathrm{~b}$} & 70 & 70.9766 & 80.8403 & 75.6130 & 72,6112 & 53.00 & 47.00 & 64.74 & 35.26 & \\
\hline & T3 & 87.3244 & 99.3778 & 92.9329 & 89.3046 & 53.47 & 46.53 & 64.69 & 35.31 & \\
\hline & T9 & 89.7526 & 97.7125 & 93.4758 & 91.0425 & 53.23 & 46.77 & 65.36 & 34.64 & \\
\hline \multicolumn{6}{|c|}{ 4b Mean } & 53.23 & 46.77 & 64.93 & 35.07 & 36.1 \\
\hline \multirow{2}{*}{\multicolumn{8}{|c|}{\begin{tabular}{|l} 
4b St. Dev. \\
Recipe 4 Mean \\
\end{tabular}}} & 0.37 & 0.37 & 0.37 \\
\hline & & & & & & & & 64.47 & & 35.8 \\
\hline \multicolumn{8}{|c|}{ Recipe 4 Mean St. Dev. (between 4a and 4b) } & 0.64 & & 0.36 \\
\hline
\end{tabular}


Tested October 31, 2001

$\% \mathrm{OC}=(100-\% \mathrm{FS}) / 1.8$

\begin{tabular}{|c|c|c|c|c|c|c|c|c|c|c|}
\hline Reactor & Crucible \# & Tare only (g) & Tare+Material (g) & After 1030 (g) & After $550^{\circ} \mathrm{C}(\mathrm{g})$ & $\% \mathrm{MC}$ & $\%$ TS & $\%$ VS & \%FS & $\% \mathrm{OC}(\mathrm{db})$ \\
\hline \multirow[t]{3}{*}{$5 a$} & T12 & 88.1906 & 101.7646 & 93.5985 & 90.2244 & 60.16 & 39.84 & 62.39 & 37.61 & \\
\hline & $T 4$ & 87.4935 & 100.1876 & 92.6012 & 89.3975 & 59.76 & 40.24 & 62.72 & 37.28 & \\
\hline & T6 & 88.2116 & 102.0984 & 93.6976 & 90.2240 & 60.49 & 39.51 & 63.32 & 36.68 & \\
\hline \multicolumn{6}{|c|}{5 a Mean } & 60.14 & 39.86 & 62.81 & 37.19 & 34.9 \\
\hline \multicolumn{6}{|c|}{ 5a St. Dev. } & 0.37 & 0.37 & 0.47 & 0.47 & 0.47 \\
\hline \multirow[t]{3}{*}{$5 b$} & $T H$ & 87.8896 & 99.8980 & 92.4934 & 89.7191 & 61.66 & 38.34 & 60.26 & 39.74 & \\
\hline & $\mathrm{A}-12$ & 94.9554 & 109.1740 & 100.4936 & 97.1640 & 61.05 & 38.95 & 60.12 & 39.88 & \\
\hline & 38 & 83.9456 & 97.4978 & 89.1888 & 86.0114 & 61.31 & 38.69 & 60,60 & 39.40 & \\
\hline \multicolumn{6}{|c|}{$5 \mathrm{~b}$ Mean } & 61.34 & 38.66 & 60.33 & 39.67 & 33.5 \\
\hline $5 \mathrm{~b}$ St. De & & & & & & 0.31 & 0.31 & 0.25 & 0.25 & 0,25 \\
\hline Recipe 5 & & & & & & & & 61.57 & & 34.2 \\
\hline \multicolumn{8}{|c|}{ Recipe 5 St. Dev. (between $5 \mathrm{a}$ and $5 \mathrm{~b}$ ) } & 1.76 & & 0.98 \\
\hline
\end{tabular}

\begin{tabular}{|c|c|c|c|c|c|c|c|c|c|c|}
\hline Reactor & Crucible \# & Tare only $(g)$ & Tare+Material (g) & After $103^{\circ} \mathrm{C}(\mathrm{g})$ & After $550^{\circ} \mathrm{C}(\mathrm{g})$ & $\% M C$ & \%TS & $\% \mathrm{VS}$ & \%FS & $\% O C$ (db) \\
\hline \multirow{3}{*}{$6 a$} & 30 & 94.1455 & 107.6910 & 98.0477 & 95.8625 & 71.19 & 28.81 & 56.00 & 44,00 & \\
\hline & $J-6$ & 81.6067 & 93.8590 & 85.2008 & 83.1730 & 70.67 & 29.33 & 56.42 & 43.58 & \\
\hline & 7 & 89.2954 & 102.4120 & 93.0221 & 90.9362 & 71.59 & 28.41 & 55.97 & 44.03 & \\
\hline \multicolumn{6}{|c|}{ 6a Mean } & 71.15 & 28.85 & 56.13 & 43.87 & 31.2 \\
\hline \multicolumn{6}{|c|}{ Ga St. Dev. } & 0.46 & 0.46 & 0.25 & 0.25 & 0.25 \\
\hline \multirow[t]{3}{*}{$6 b$} & $\sqrt{-4}$ & 90.8554 & 101.1469 & 93.7790 & 92.1192 & 71.59 & 28.41 & 56.77 & 43.23 & \\
\hline & 3 & 90.9391 & 105.1501 & 94.9020 & 92.6646 & 72.11 & 27.89 & 56.46 & 43.54 & \\
\hline & 74 & 85.5387 & 101.2220 & 89.8895 & 87.4177 & 72.26 & 27.74 & 56.81 & 43.19 & \\
\hline \multicolumn{6}{|c|}{$6 \mathrm{~b}$ Mean } & 71.99 & 28.01 & 56.68 & 43.32 & 31.5 \\
\hline \multirow{2}{*}{\multicolumn{8}{|c|}{$\begin{array}{l}\text { 6b St. Dev. } \\
\text { Reclpe } 6 \text { Mean }\end{array}$}} & 0.19 & 0.19 & 0.19 \\
\hline & & & & & & & & 56.41 & & 31.3 \\
\hline \multicolumn{8}{|c|}{$\begin{array}{l}\text { Recipe b Mean } \\
\text { Recipe } 6 \text { St. Dev. (between } 6 a \text { and } 6 b)\end{array}$} & 0.39 & & 0.22 \\
\hline
\end{tabular}

\begin{tabular}{|c|c|c|c|c|c|c|c|c|c|c|}
\hline Reactor & Crucible\# & Tare only (g) & Tare+Material (g) & After $103^{\circ} \mathrm{C}(\mathrm{g})$ & After $550^{\circ} \mathrm{C}(\mathrm{g})$ & $\% \mathrm{MC}$ & \%TS & \%VS & \%FS & $\% 0 \mathrm{O}(\mathrm{db})$ \\
\hline \multirow[t]{3}{*}{$7 \mathrm{a}$} & $T_{2}$ & 88.1442 & 97.8504 & 91.9413 & 89.7946 & 60.88 & 39.12 & 56.54 & 43.46 & \\
\hline & 50 & 90.7192 & 99.2448 & 94.0512 & 92.1680 & 60.92 & 39.08 & 56.52 & 43.48 & \\
\hline & $H 1$ & 90.8016 & 100.0721 & 94.3661 & 92.3527 & 61.55 & 38.45 & 56.48 & 43.52 & \\
\hline \multicolumn{6}{|l|}{$7 a$ Mean } & 61.12 & 38.88 & 56.51 & 43.49 & 31.4 \\
\hline \multicolumn{6}{|c|}{ 7a St. Dav. } & 0.38 & 0.38 & 0.03 & 0.03 & 0.03 \\
\hline \multirow[t]{3}{*}{$7 \mathrm{~b}$} & 49 & 90.8470 & 101.5844 & 95.2017 & 92.7013 & 60.00 & 40.00 & 58.77 & 41.23 & \\
\hline & A-7 & 91.3097 & 104.3000 & 96.5408 & 93.5024 & 59.73 & 40.27 & 58.08 & 41.92 & \\
\hline & $A-8$ & 89.8043 & 101.3888 & 94.4218 & 91.7258 & 60.14 & 39.86 & 58.39 & 41.61 & \\
\hline \multicolumn{6}{|c|}{$7 \mathrm{~b}$ Mean } & 59.96 & 40.04 & 58.41 & 41.59 & 32.5 \\
\hline \multicolumn{6}{|c|}{ 7b St. Dev. } & 0.21 & 0.21 & 0.34 & 0.34 & 0.34 \\
\hline \multicolumn{8}{|c|}{\begin{tabular}{|l|l|} 
Recipe 7 Mean & \\
\end{tabular}} & 57.46 & & 31.9 \\
\hline \multicolumn{8}{|c|}{ Recipe 7 St. Dev. (between 7a and 7b) } & 1.34 & & 0.75 \\
\hline
\end{tabular}


Phase 1 - Volatile Solids Summary

Reactor Basis (standard deviations are for triplicate samples)

\begin{tabular}{|c|c|c|c|c|c|c|c|}
\hline Reactor & Initial \%VS & $\begin{array}{l}\text { Tnitial Dry } \\
\text { Weight (g) }\end{array}$ & Initial VS (g) & Final \%VS & $\begin{array}{l}\text { Final Dry } \\
\text { Weight (g) }\end{array}$ & Final VS (g) & $\begin{array}{l}\text { \% Reduction } \\
\text { of vs Mass }\end{array}$ \\
\hline $1 \mathrm{a}$ & 82.24 & 90.12 & 74.12 & 73.50 & 54.63 & \begin{tabular}{|l}
40.15 \\
\end{tabular} & 45.8 \\
\hline st.dev. & 0.93 & & 0.93 & 0.41 & & 0.41 & \\
\hline $1 \mathrm{~b}$ & 82.12 & 90.03 & 73.93 & 73.26 & 54.68 & 40.06 & 45.8 \\
\hline st.dev. & 0.83 & & 0.83 & 0.52 & & 0.52 & \\
\hline $2 a$ & 80.53 & 80.27 & 64.64 & 66.33 & 42.12 & 27.94 & 56.8 \\
\hline st.dev. & 1.88 & & 1.88 & 0.49 & & 0.49 & \\
\hline $2 b$ & 79.10 & 80.51 & 63.68 & 64.23 & 39.98 & 25.68 & 59.7 \\
\hline st.dev. & 0.34 & & 0.34 & 0.25 & & 0.25 & \\
\hline $3 a$ & 80.95 & 152.62 & 123.55 & 70.05 & 96.34 & 67.49 & 45.4 \\
\hline st.dev. & 0.61 & & 0.61 & 0.14 & & 0.14 & \\
\hline $3 \mathrm{~b}$ & 80.41 & 155.06 & 124.68 & 70.13 & 91.74 & 64.34 & 48.4 \\
\hline st.dev. & 0.70 & · & 0.70 & 0.40 & & 0.40 & \\
\hline $4 a$ & 80.01 & 114.30 & 91.45 & 64.02 & 58.73 & 37.60 & 58.9 \\
\hline st.dev. & 0.56 & & 0.56 & 0.12 & & 0.12 & \\
\hline $4 \mathrm{~b}$ & 80.29 & 113.70 & 91.29 & 64.93 & 58.28 & 37.84 & 58.5 \\
\hline st.dev. & 0.35 & & 0.35 & 0.37 & & 0.37 & \\
\hline $5 a$ & 80.38 & 96.49 & 77.56 & 62.81 & 44.46 & 27.93 & 64.0 \\
\hline st.dev. & 1.01 & & 1.01 & 0.47 & & 0.47 & \\
\hline $5 b$ & 81.01 & 96.18 & 77.92 & 60.33 & 41.56 & 25.07 & 67.8 \\
\hline st.dev. & 0.34 & & 0.34 & 0.25 & & 0.25 & \\
\hline $6 a$ & 81.61 & 80.50 & 65.69 & 56.13 & 32.28 & 18.12 & 72.4 \\
\hline st.dev. & 1.74 & & 1.74 & 0.25 & & 0.25 & \\
\hline $6 \mathrm{~b}$ & 80.70 & 76.54 & 61.77 & 56.68 & 32.42 & 18.38 & 70.2 \\
\hline st.dev. & 0.49 & & 0.49 & 0.19 & & 0.19 & \\
\hline $7 a$ & 80.35 & 96.30 & 77.38 & 56.51 & 39.85 & 22.52 & 70.9 \\
\hline st.dev. & 0.83 & & 0.83 & 0.03 & & 0.03 & \\
\hline $7 b$ & 80.30 & 94.05 & 75.52 & 58.41 & 40.30 & 23.54 & 68.8 \\
\hline st.dev. & 1.48 & & 1.48 & 0.34 & & 0.34 & \\
\hline
\end{tabular}

Phase 1 - Volatlle Solids Summary

Recipe Basis (standard deviations are for duplicate reactors)

\begin{tabular}{|c|c|c|c|c|c|c|}
\hline Recipe & $\% \mathrm{MC}$ & Initial \%VS & Initial VS $(g)$ & Final \%VS & Final VS (g) & $\%$ Reduction \\
\hline $1 \mathrm{~A}$ & 57 & 82.18 & 74.03 & 73.38 & 40.10 & 45.8 \\
\hline st.dev. & & 0.09 & 0.13 & 0.17 & 0.07 & 0.0 \\
\hline 1B & 70 & 79.81 & 64.16 & 65.28 & 26.81 & 58.2 \\
\hline st.dev. & & 1.01 & 0.67 & 1.48 & 1.60 & 2.0 \\
\hline $2 \mathrm{~A}$ & 40 & 80.68 & 124.11 & 70.09 & 65.91 & 46.9 \\
\hline st.dev. & & 0.38 & 0.80 & 0.06 & 2.22 & 2.1 \\
\hline $2 \mathrm{~B}$ & 55 & 80.15 & 91.37 & 64.47 & 37.72 & 58.7 \\
\hline st.dev. & & 0.20 & 0.11 & 0.64 & 0.17 & 0.2 \\
\hline $2 \mathrm{C}$ & 62.5 & 80.70 & 77.74 & 61.57 & 26.50 & 65.9 \\
\hline st.dev. & & 0.45 & 0.25 & 1.76 & 2.02 & 2.7 \\
\hline $2 \mathrm{D}$ & 70 & 81.15 & 63.73 & 56.41 & 18.25 & 71.3 \\
\hline st.dev. & & 0.64 & 2.78 & 0.39 & 0.18 & 1.5 \\
\hline $2 \mathrm{E}$ & 62.5 & 80.33 & 76.45 & 57.46 & 23.03 & 69.9 \\
\hline st.dev. & & 0.03 & 1.31 & 1.34 & 0.72 & 1.5 \\
\hline
\end{tabular}


Phase 1 - C:N Summary

Reactor Basis

\begin{tabular}{|l|c|c|}
\hline Reactor & Initial C:N & Final C:N \\
\hline $1 \mathrm{a}$ & 31.7 & 21.8 \\
\hline $1 \mathrm{~b}$ & 31.7 & 20.0 \\
\hline $2 \mathrm{a}$ & 31.7 & 15.2 \\
\hline $2 \mathrm{~b}$ & 31.7 & 13.2 \\
\hline $3 \mathrm{a}$ & 34.7 & 22.3 \\
\hline $3 \mathrm{~b}$ & 34.7 & 22.2 \\
\hline $4 \mathrm{a}$ & 34.7 & 17.6 \\
\hline $4 \mathrm{~b}$ & 34.7 & 17.6 \\
\hline $5 \mathrm{a}$ & 34.7 & 16.5 \\
\hline $5 \mathrm{~b}$ & 34.7 & 14.5 \\
\hline $6 \mathrm{a}$ & 34.7 & 12.4 \\
\hline $6 \mathrm{~b}$ & 34.7 & 12.2 \\
\hline $7 \mathrm{a}$ & 28.8 & 12.3 \\
\hline $7 \mathrm{~b}$ & 28.8 & 13.2 \\
\hline
\end{tabular}

Recipe Basis

(standard deviations are for duplicate reactors)

\begin{tabular}{|c|c|c|}
\hline Recipe & Initial C:N & Final $\mathrm{C}: \mathrm{N}$ \\
\hline $1 \mathrm{~A}$ & 31.7 & 20.9 \\
\hline st.dev. & 0 & 1.3 \\
\hline $1 B$ & 31.7 & 14.2 \\
\hline st.dev. & 0 & 1.4 \\
\hline $2 A$ & 34.7 & 22.3 \\
\hline st.dev. & 0 & 0.1 \\
\hline $2 B$ & 34.7 & 17.6 \\
\hline st.dev. & 0 & 0.0 \\
\hline $2 \mathrm{C}$ & 34.7 & 15.5 \\
\hline st.dev. & 0 & 1.4 \\
\hline $2 \mathrm{D}$ & 34.7 & 12.3 \\
\hline st.dev. & 0 & 0.1 \\
\hline $2 E$ & 28.8 & 12.8 \\
\hline st.dev. & 0 & 0.6 \\
\hline
\end{tabular}


Phase 1 - Organic Carbon Summary

Reactor Basis (standard deviations are for triplicate samples)

\begin{tabular}{|c|c|c|c|c|c|c|c|}
\hline Beactor & |initial \%OC & $\begin{array}{l}\text { Initial Dry } \\
\text { Weight (g) }\end{array}$ & $\begin{array}{c}\text { Initial mass } \\
\mathrm{OC}(\mathrm{g})\end{array}$ & Final \%OC & $\begin{array}{c}\text { Final Dry } \\
\text { Weight (g) }\end{array}$ & $\begin{array}{c}\text { Final mass } \\
\mathrm{OC}(\mathrm{g})\end{array}$ & $\begin{array}{c}\text { \% Reduction } \\
\text { in Mass of } \\
\text { OC }\end{array}$ \\
\hline $1 \mathrm{a}$ & 45.69 & 90.12 & 41.18 & 40.83 & 54.63 & 22.31 & $\begin{array}{l}45.8 \\
\end{array}$ \\
\hline st.dev. & 0.93 & & & 0.41 & & & \\
\hline $1 \mathrm{~b}$ & 45.62 & 90.03 & 41.07 & 40.70 & 54.68 & 22.25 & 45.8 \\
\hline st.dev. & 0.83 & & & 0.52 & & & \\
\hline $2 a$ & 44.74 & 80.27 & 35.91 & 36.85 & 42.12 & 15.52 & 56.8 \\
\hline st.dev. & 1.88 & & & 0.49 & & & \\
\hline $2 b$ & 43.95 & 80.51 & 35.38 & 35.69 & 39.98 & 14.27 & 59.7 \\
\hline st.dev. & 0.34 & & & 0.25 & & & \\
\hline $3 a$ & 44.97 & 152.62 & 68.64 & 38.92 & 96.34 & 37.49 & 45.4 \\
\hline st.dev. & 0.61 & & & 0.14 & & & \\
\hline $3 \mathrm{~b}$ & 44.67 & 155.06 & 69.27 & 38.96 & 91.74 & 35.74 & 48.4 \\
\hline st.dev. & 0.70 & & & 0.40 & & & \\
\hline $4 a$ & 44.45 & 114.30 & 50.80 & 35.57 & 58.73 & 20.89 & 58.9 \\
\hline st.dev. & 0.56 & & & 0.12 & & & \\
\hline $4 b$ & 44.61 & 113.70 & 50.72 & 36.07 & 58.28 & 21.02 & 58.5 \\
\hline st.dev. & 0.35 & & & 0.37 & & & \\
\hline $5 a$ & 44.66 & 96.49 & 43.09 & 34.89 & 44.46 & 15.51 & 64.0 \\
\hline st.dev. & 1.01 & & & 0.47 & & & \\
\hline $5 b$ & 45.01 & 96.18 & 43.29 & 33.52 & 41.56 & 13.93 & 67.8 \\
\hline st.dev. & 0.34 & & & 0.25 & & & \\
\hline $6 a$ & 45.34 & 80.50 & 36.50 & 31.18 & 32.28 & 10.07 & 72.4 \\
\hline st.dev. & 1.74 & & & 0.25 & & & \\
\hline $6 b$ & 44.83 & 76.54 & 34.31 & 31.49 & 32.42 & 10.21 & 70.2 \\
\hline st.dev. & 0.49 & & & 0.19 & & & \\
\hline $7 \mathrm{a}$ & 44.64 & 96.30 & 42.99 & 31.40 & 39.85 & 12.51 & 70.9 \\
\hline st.dev. & 0.83 & & & 0.03 & & & \\
\hline $7 \mathrm{~b}$ & 44.61 & 94.05 & 41.96 & 32.45 & 40.30 & 13.08 & 68.8 \\
\hline st.dev. & 1.48 & & & 0.34 & & & \\
\hline
\end{tabular}

Phase 1 - Organic Carbon Summary

Recipe Basis (standard deviations are for duplicate reactors)

\begin{tabular}{|c|c|c|c|c|c|}
\hline Recipe & Initial \%OC & $\begin{array}{l}\text { Initial mass } \\
O C(g)\end{array}$ & Final $\% O C$ & $\begin{array}{c}\text { Final mass } O C \\
(\mathrm{~g})\end{array}$ & $\%$ Reduction \\
\hline $1 \mathrm{~A}$ & 45.66 & 41.13 & 40.77 & 22.28 & 45.8 \\
\hline st.dev. & 0.05 & 0.07 & 0.09 & 0.04 & 0.0 \\
\hline $1 \mathrm{~B}$ & 44.34 & 35.65 & 36.27 & 14.89 & 58.2 \\
\hline st.dev. & 0.56 & 0.37 & 0.82 & 0.89 & 2.0 \\
\hline $2 \mathrm{~A}$ & 44.82 & 68.95 & 38.94 & 36.62 & 46.9 \\
\hline st.dev. & 0.21 & 0.45 & 0.03 & 1.24 & 2.1 \\
\hline $2 \mathrm{~B}$ & 44.53 & 50.76 & 35.82 & 20.96 & 58.7 \\
\hline st.dev. & 0.11 & 0.06 & 0.36 & 0.10 & 0.2 \\
\hline $2 \mathrm{C}$ & 44.83 & 43.19 & 34.21 & 14.72 & 65.9 \\
\hline st.dev. & 0.25 & 0.14 & 0.98 & 1.12 & 2.7 \\
\hline $2 \mathrm{D}$ & 45.08 & 35.41 & 31.34 & 10.14 & 71.3 \\
\hline st.dev. & 0.36 & 1.54 & 0.22 & 0.10 & 1.5 \\
\hline $2 \mathrm{E}$ & 44.63 & 42.47 & 31.92 & 12.79 & 69.9 \\
\hline st.dev. & 0.02 & 0.73 & 0.75 & 0.40 & 1.5 \\
\hline
\end{tabular}




\begin{tabular}{|c|c|c|c|c|c|c|c|}
\hline Reactor & Start \%N & $\begin{array}{c}\text { Start Dry } \\
\text { Weight }(\mathrm{g})\end{array}$ & $\begin{array}{c}\text { Start, mass N } \\
(\mathrm{g})\end{array}$ & Finish \%N & $\begin{array}{l}\text { Finish Dry } \\
\text { Weight (g) }\end{array}$ & $\begin{array}{c}\text { Finish, mass } \\
N(g)\end{array}$ & $\begin{array}{l}\% \text { Loss in } \\
\text { mass of } N\end{array}$ \\
\hline $1 \mathrm{a}$ & 1.43 & 90.12 & 1.29 & 1.87 & 54.63 & 1.02 & 20.7 \\
\hline st.dev. & 0.23 & & & 0.13 & & & \\
\hline $1 b$ & 1.43 & 90.03 & 1.29 & 2.04 & 54.68 & 1.12 & 13.4 \\
\hline st.dev. & 0.23 & & & 0.11 & & & \\
\hline $2 a$ & 1.43 & 80.27 & 1.15 & 2.43 & 42.12 & 1.02 & 10.8 \\
\hline st.dev. & 0.23 & & & 0.13 & & & \\
\hline $2 \mathrm{~b}$ & 1.43 & 80.51 & 1.15 & 2.70 & 39.98 & 1.08 & 6.2 \\
\hline st.dev. & 0.23 & & & 0.36 & & & \\
\hline $3 a$ & 1.29 & 152.62 & 1.97 & 1.74 & 96.34 & 1.68 & 14.9 \\
\hline st.dev. & 0.13 & & & 0.08 & & & \\
\hline $3 b$ & 1.29 & 155.06 & 2.00 & 1.76 & 91.74 & 1.61 & 19.3 \\
\hline st.dev. & 0.13 & & & 0.03 & & & \\
\hline $4 \mathrm{a}$ & 1.29 & 114.30 & 1.47 & 2.03 & 58.73 & 1.19 & 19.1 \\
\hline st.dev. & 0.13 & & & 0.06 & & & \\
\hline $4 b$ & 1.29 & 113.70 & 1.47 & 2.05 & 58.28 & 1.19 & 18.5 \\
\hline st.dev. & 0.13 & & & 0.04 & & & \\
\hline $5 a$ & 1.29 & 96.49 & 1.24 & 2.12 & 44.46 & 0.94 & 24.3 \\
\hline st.dev. & 0.13 & & & 0.05 & & & \\
\hline $5 b$ & 1.29 & 96.18 & 1.24 & 2.31 & 41.56 & 0.96 & 22.6 \\
\hline st.dev. & 0.13 & & & 0.04 & & & \\
\hline $6 \mathrm{a}$ & 1.29 & 80.50 & 1.04 & 2.51 & 32.28 & 0.81 & 22.0 \\
\hline st.dev. & 0.13 & & & 0.05 & & & \\
\hline $6 b$ & 1.29 & 76.54 & 0.99 & 2.58 & 32.42 & 0.84 & 15.3 \\
\hline st.dev. & 0.13 & & & 0.07 & & & \\
\hline $7 \mathrm{a}$ & 1.55 & 96.30 & 1.49 & 2.53 & 39.85 & 1.01 & 32.5 \\
\hline st.dev. & 0.18 & & & 0.03 & & & \\
\hline $7 \mathrm{~b}$ & 1.55 & 94.05 & 1.46 & 2.45 & 40.30 & 0.99 & 32.3 \\
\hline st.dev. & 0.18 & & & 0.11 & & & \\
\hline
\end{tabular}

Phase 1 - \% Nitrogen Summary

Reclpe Basis (standard deviations are for duplicate reactors)

\begin{tabular}{|c|c|c|c|c|c|c|c|}
\hline Recipe & Set \%MC & Start C:N & Start $\% \mathrm{~N}$ & $\begin{array}{c}\text { Start, mass N } \\
\text { (g) }\end{array}$ & Finish \%N & $\begin{array}{c}\text { Finish, mass } \\
N(g)\end{array}$ & $\begin{array}{l}\% \text { Loss in } \\
\text { mass of } N\end{array}$ \\
\hline $1 \mathrm{~A}$ & 57 & 32.0 & 1.43 & 1.29 & 1.96 & 1.07 & 17.0 \\
\hline st.dev. & & & 0.23 & 0.00 & 0.14 & 0.07 & 5.2 \\
\hline 18 & 70 & 31.0 & 1.43 & 1.15 & 2.57 & 1.05 & 8.5 \\
\hline st.dev. & & & 0.23 & 0.00 & 0.28 & 0.04 & 3.2 \\
\hline $2 A$ & 40 & 34.7 & 1.29 & 1.98 & 1.75 & 1.65 & 17.1 \\
\hline st.dev. & & & 0.13 & 0.02 & 0.05 & 0.04 & 3.1 \\
\hline $2 B$ & 55 & 34.5 & 1.29 & 1.47 & 2.04 & 1.19 & 18.8 \\
\hline st.dev. & & & 0.13 & 0.01 & 0.05 & 0.00 & 0.4 \\
\hline $2 \mathrm{C}$ & 62.5 & 34.7 & 1.29 & 1.24 & 2.21 & 0.95 & 23.4 \\
\hline st.dev. & & & 0.13 & 0.00 & 0.11 & 0.01 & 1.2 \\
\hline $2 \mathrm{D}$ & 70 & 35.0 & 1.29 & 1.01 & 2.54 & 0.82 & 18.6 \\
\hline st.dev. & & & 0.13 & 0.04 & 0.07 & 0.02 & 4.7 \\
\hline $2 E$ & 62.5 & 28.8 & 1.55 & 1.48 & 2.49 & 1.00 & 32.4 \\
\hline st.dev. & & & 0.18 & 0.02 & 0.08 & 0.01 & 0.1 \\
\hline
\end{tabular}


Reactor Weights

\section{Reactor Basis}

\begin{tabular}{|l|c|}
\hline Reactor & $\%$ Reduction \\
\hline $1 a$ & 39.4 \\
\hline $1 b$ & 39.3 \\
\hline $2 a$ & 47.5 \\
\hline $2 b$ & 50.3 \\
\hline $3 a$ & 36.9 \\
\hline $3 b$ & 40.8 \\
\hline $4 a$ & 48.6 \\
\hline $4 b$ & 48.7 \\
\hline $5 a$ & 53.9 \\
\hline $5 b$ & 56.8 \\
\hline $6 a$ & 59.9 \\
\hline $6 b$ & 57.6 \\
\hline $7 a$ & 58.6 \\
\hline $7 b$ & 57.2 \\
\hline
\end{tabular}

Recipe Basis (standard deviations are for duplicate reactors)

\begin{tabular}{|c|c|c|}
\hline Recipe & \% Reduction & st.dev. \\
\hline $1 \mathrm{~A}$ & 39.3 & 0.1 \\
\hline $1 \mathrm{~B}$ & 48.9 & 2.0 \\
\hline $2 \mathrm{~A}$ & 38.9 & 2.8 \\
\hline $2 \mathrm{~B}$ & 48.7 & 0.1 \\
\hline $2 \mathrm{C}$ & 55.4 & 2.0 \\
\hline $2 \mathrm{D}$ & 58.8 & 1.6 \\
\hline $2 \mathrm{E}$ & 57.9 & 1.0 \\
\hline
\end{tabular}



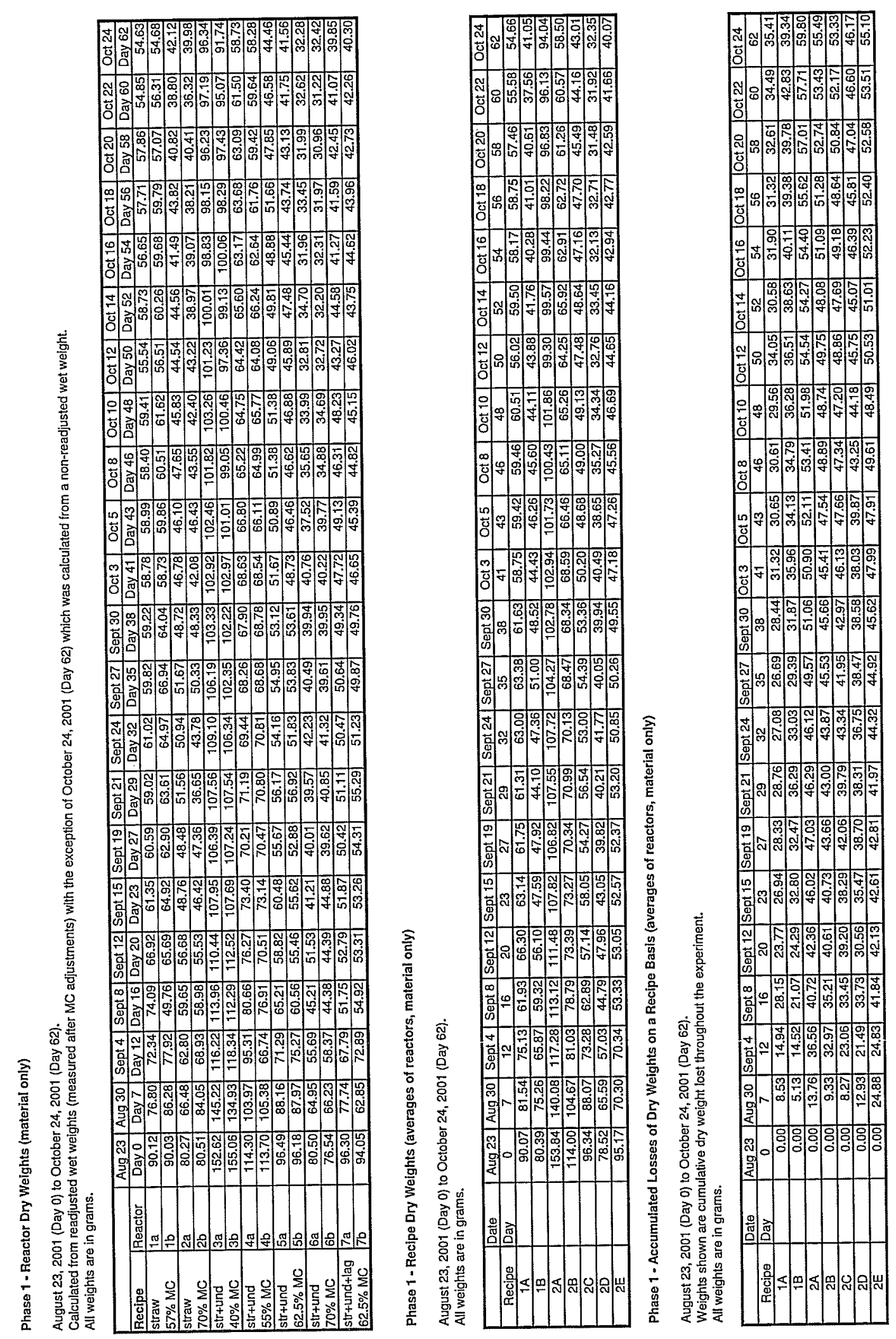
Phase 1 - TKN Analysis for Feedstock Materials (July 4)

Standards:
\begin{tabular}{|c|c|}
\hline $\mathrm{mg} \mathrm{N} / \mathrm{L}$ & \# of squares \\
\hline 75 & 79.5 \\
\hline 50 & 68 \\
\hline 25 & 40 \\
\hline 10 & 18.5 \\
\hline 5 & 11 \\
\hline
\end{tabular}

Conversion Equation:

\# squares $/ 1.1946=\mathrm{mg} \mathrm{N} / \mathrm{L}$

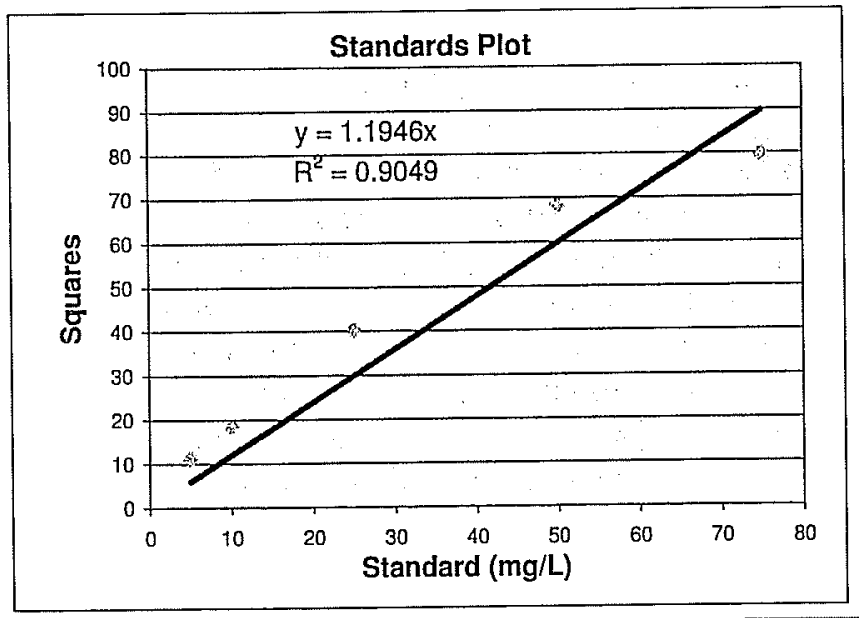

Straw

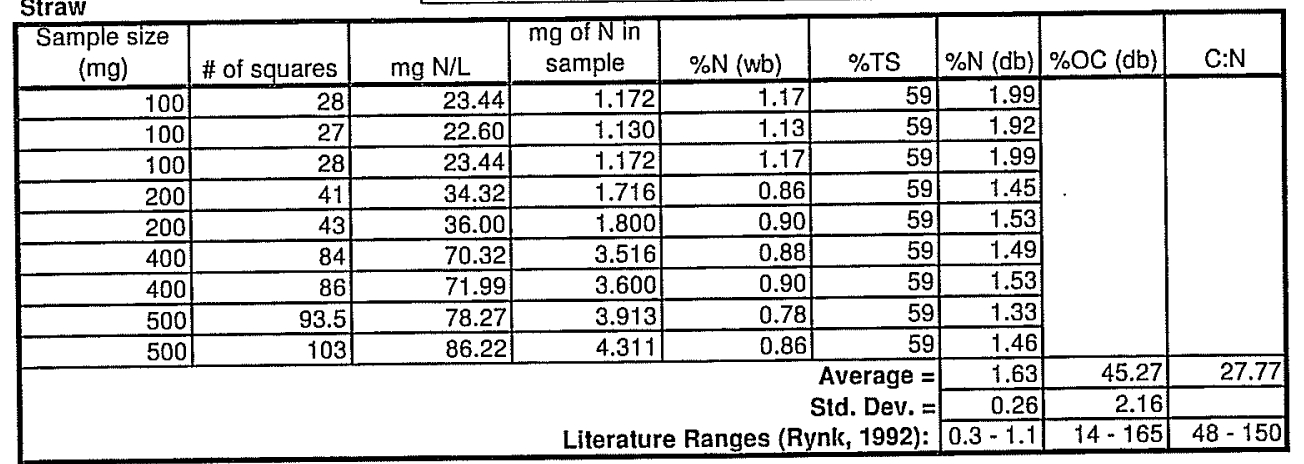

Notes:

1) Samples collected on June 18, 2001.

2) Analysis completed on July 4, 2001.

3) Used wet straw samples from Bale $3(\mathrm{MC}=41 \%)$ and calculated $\% \mathrm{~N}(\mathrm{db})$ using TS of material.

\begin{tabular}{|c|c|c|c|c|c|c|c|c|}
\hline $\begin{array}{l}\text { Sample Size } \\
(\mathrm{mL})\end{array}$ & \# of squares & 1:5 dilution & $\mathrm{mg} \mathrm{N} / \mathrm{L}$ & $\%$ TS & $\% N(w b)$ & $\% \mathrm{~N}(\mathrm{db})$ & $\% O C(\mathrm{db})$ & C:N \\
\hline 10 & 49 & 245 & 1025.45 & 6.9 & 1.49 & 21.54 & & \multirow{4}{*}{ · } \\
\hline 10 & 53 & 265 & 1109.16 & 6.9 & 1.61 & 23.30 & & \\
\hline 10 & 47 & 235 & 983.59 & 6.9 & 1.43 & 20.66 & & \\
\hline 10 & 48 & 240 & 1004.52 & 6.9 & 1.46 & 21.10 & & \\
\hline \multirow{3}{*}{\multicolumn{5}{|c|}{$\begin{array}{r}\text { Average }= \\
\text { Std. Dev. }= \\
31,2000)\end{array}$}} & 1.49 & 21.65 & 13.89 & 0.64 \\
\hline & & & & & 0.08 & 1.16 & 1.5 & \\
\hline & & & & & 0.35 & 10.6 & & \\
\hline
\end{tabular}

Notes:

1) Samples coilected on June 18, 2001.

2) Analysis completed on July $4,2001$.

3) $\% \mathrm{~N}$ (wb) calculation: $1 \%$ TS $=10,000 \mathrm{mg} / \mathrm{L}$.

4) Used liquid lagoon sample with $M C=93.1 \%$. 
Phase 1 - TKN Analysis for Feedstock Materials

Standards:
\begin{tabular}{|c|c|}
\hline mg N/L & \# of squares \\
\hline 75 & 106 \\
\hline 50 & 73 \\
\hline 25 & 39 \\
\hline 10 & 24 \\
\hline 5 & 7 \\
\hline
\end{tabular}

Conversion Equation:

\# squares $/ 1.4479=\mathrm{mg} \mathrm{N} / \mathrm{L}$

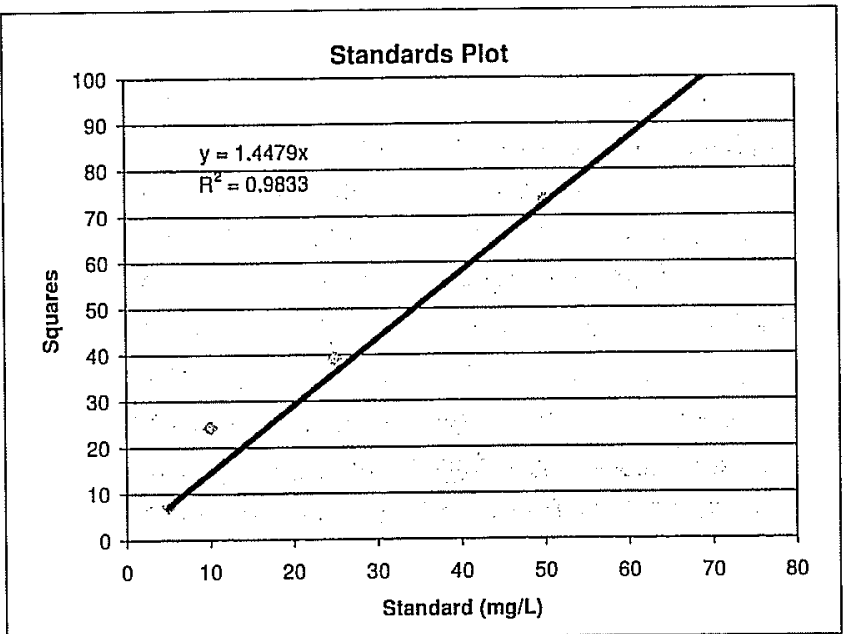

Straw

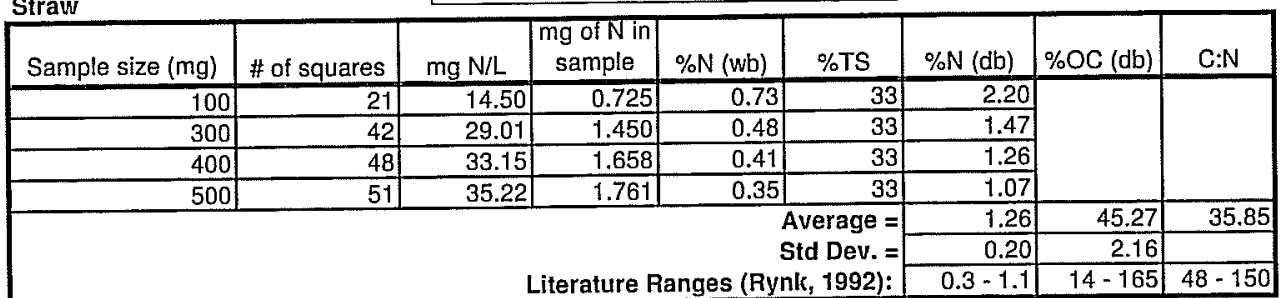

Notes:

1) Samples collected on June 18, 2001.

2) Analysis completed on August 20, 2001.

3) Used wet straw samples from Bale $3(\mathrm{MC}=41 \%)$ and calculated $\% \mathrm{~N}(\mathrm{db})$ using TS of material.

4) $\% \mathrm{~N}$ calculated using TS of material.

Lagoon Mixture

\begin{tabular}{|c|c|c|c|c|c|c|c|c|}
\hline Sample size $(\mathrm{mL})$ & \# of squares & T:10 dilution & $\mathrm{mg} \mathrm{N} / \mathrm{L}$ & \%TS & $\% \mathrm{~N}(\mathrm{wb})$ & $\% \mathrm{~N}(\mathrm{db})$ & $\% O C(d b)$ & $\mathrm{G}: \mathrm{N}$ \\
\hline 10 & 45 & 450 & 1553.97 & 6.9 & 2.25 & 32.64 & & \\
\hline 10 & 70 & 700 & 2417.29 & 6.9 & 3.50 & 50.77 & & \\
\hline 10 & 43 & 430 & 1484.91 & 6.9 & 2.15 & 31.19 & & \\
\hline \multirow{2}{*}{\multicolumn{5}{|c|}{$\begin{aligned} \text { Average } & = \\
\text { Std Dev. }= & \end{aligned}$}} & 2.64 & 38.20 & 13.89 & 0.36 \\
\hline & & & & & 0.75 & 10.91 & 2.69 & \\
\hline \multicolumn{5}{|c|}{ Enviro-Test Laboratories Analysis Report (October 31,2000 ): } & 0.35 & 10.6 & & \\
\hline
\end{tabular}

Notes:

1) Samples collected on June 18, 2001.

2) Analysis completed on August 20, 2001.

3) $\% \mathrm{~N}(\mathrm{wb})$ calculation: $1 \% \mathrm{TS}=10,000 \mathrm{mg} / \mathrm{L}$.

4) Used liquid lagoon sample with $\mathrm{MC}=93.1 \%$ 
Phase 1 - TKN Analysis for Feedstock Materials - Summary

\begin{tabular}{|c|c|c|c|c|c|c|}
\hline \\
\hline Date Tested & $\% \mathrm{MC}$ & $\%$ TS & & $\% \mathrm{~N}(\mathrm{db})$ & $\% O C(d b)$ & $\mathrm{C:N}$ \\
\hline July 4 & 41 & 59 & Average & 1.59 & 45.27 & 28.50 \\
\hline & & & St. Dev. & 0.25 & 2.16 & \\
\hline August 20 & 67 & 33 & Average & 1.26 & 45.27 & 35.85 \\
\hline & Litera & $\operatorname{ann}$ & $\frac{\text { St. Dev. }}{\text { k, 1992): }}$ & $\frac{0.20}{0.3-1.1}$ & $\frac{1.70}{14-165}$ & \\
\hline
\end{tabular}

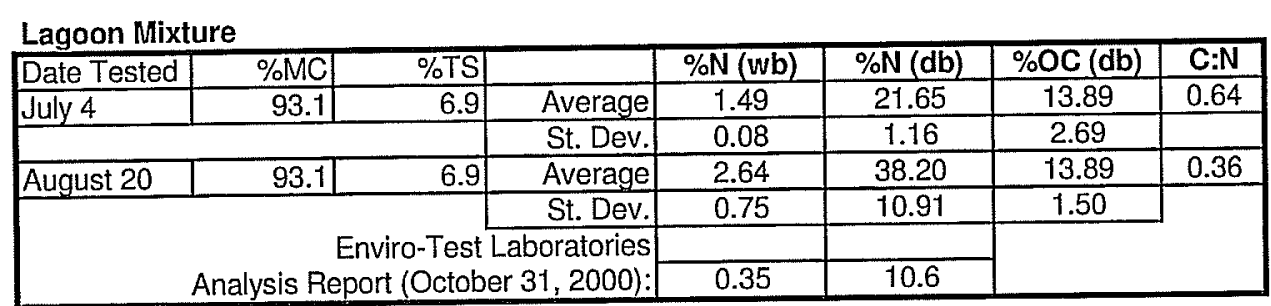

Values Used for Phase 1:

\begin{tabular}{|l|r|c|c|c|c|}
\hline Material & & $\% M C$ & $\% N(d b)$ & $\% O C(d b)$ & $C: N$ \\
\hline \multirow{4}{*}{ Straw } & Value: & 57 & 1.43 & 45.27 & 31.74 \\
\cline { 2 - 6 } & Std. Dev.: & 16 & 0.22 & 2.16 & \\
\cline { 2 - 6 } & Value: & 93.1 & 23.48 & 13.89 & 0.59 \\
\cline { 2 - 6 } & Std. Dev.: & 0.2 & 13.75 & & \\
\hline
\end{tabular}


Phase 1 - Initlal TKN Analysis

Standards
std cal = 3.0
\begin{tabular}{|c|c|}
\hline $\mathrm{mg} \mathrm{N} / \mathrm{L}$ & \# of squares \\
\hline 75 & 70 \\
\hline 50 & 55 \\
\hline 25 & 30 \\
\hline 10 & 20 \\
\hline 5 & 7 \\
\hline squares $/ 1.0124=\mathrm{mg} \mathrm{N} / \mathrm{L}$
\end{tabular}
S+U\%MC =
\%TS =
S+U+L \%MC =
$\% \mathrm{TS}=$

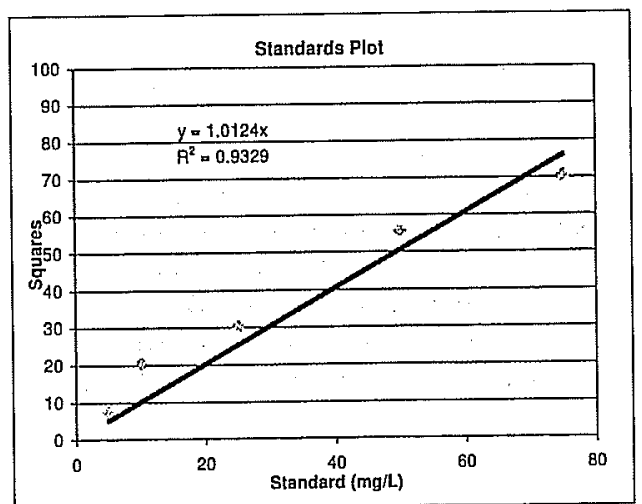

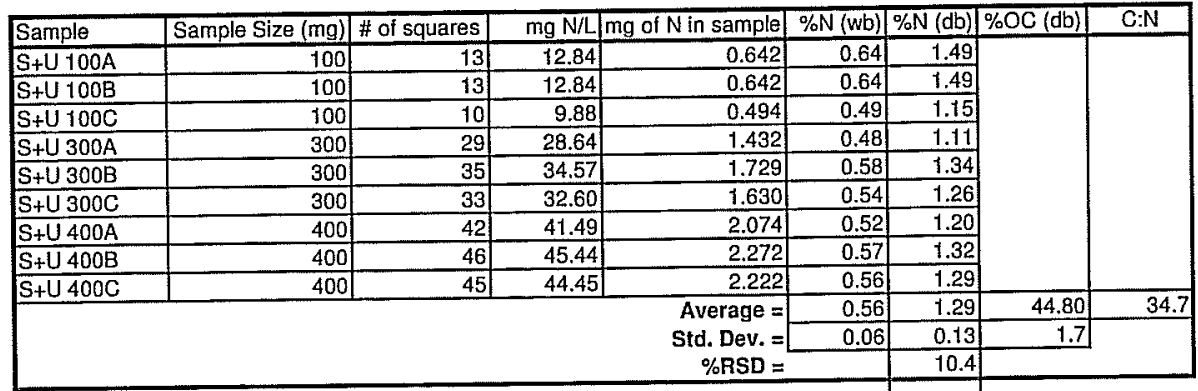

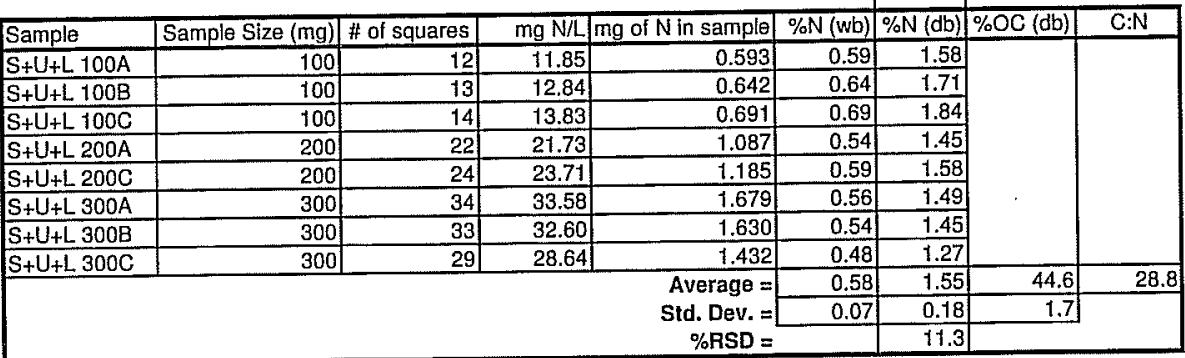

Noles:

1) Analysis completed on Seplember 13, 2001.

2) $S+U=$ straw and under recipe.

3) $S+U+L=$ straw and under and lagoon mixlure recipe. 
Phase 1 - Final TKN Analysis

Standards

std $\mathrm{cal}=3.0$

\begin{tabular}{|c|c|}
\hline $\mathrm{mg} \mathrm{N} / \mathrm{L}$ & \# of squares \\
\hline 75 & 69 \\
\hline 50 & 49 \\
\hline 25 & 24 \\
\hline 10 & \\
\hline 5 & 5 \\
\hline
\end{tabular}

Conversion Equation: \# squares $/ 0.9402=\mathrm{mg} \mathrm{N} / \mathrm{L}$

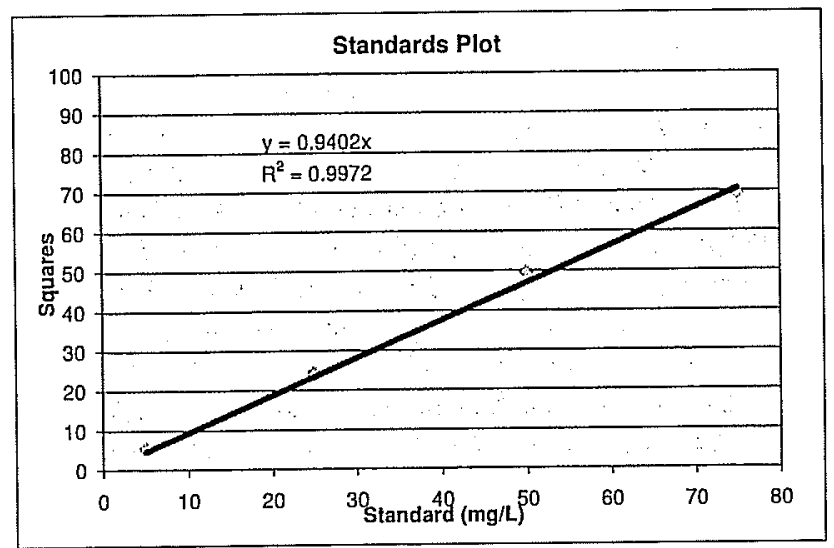

\begin{tabular}{|c|c|c|c|c|c|c|c|c|c|}
\hline Reactor & Sample & $\begin{array}{l}\text { Sample Size } \\
\text { (mg) }\end{array}$ & $\begin{array}{c}\text { \# of } \\
\text { squares }\end{array}$ & $\mathrm{mg} N / L$ & $\begin{array}{c}\mathrm{mg} \text { of } \mathrm{N} \text { in } \\
\text { sample }\end{array}$ & $\% N(w b)$ & $\% \mathrm{MC}$ & $\%$ TS & $\% N(d b)$ \\
\hline $1 \mathrm{a}$ & 1 & 400 & 65 & 69.13 & 3.457 & 0.86 & 52 & 48 & 1.80 \\
\hline $1 \mathrm{a}$ & ii & 400 & 73 & 77.64 & 3.882 & 0.97 & 52 & 48 & 2.02 \\
\hline $1 \mathrm{a}$ & iii & 400 & 65 & 69.13 & 3.457 & 0.86 & 52 & 48 & 1.80 \\
\hline \multicolumn{6}{|l|}{ 1a Mean } & 0.90 & & & 1.87 \\
\hline \multicolumn{6}{|c|}{ ta St. Dev. } & 0.06 & & & 0.13 \\
\hline $1 b$ & $i$ & 400 & 76 & 80.83 & 4.042 & 1.01 & 53 & 47 & 2.15 \\
\hline $1 \mathrm{~b}$ & ii & 400 & 72 & 76.58 & 3.829 & 0.96 & 53 & 47 & 2.04 \\
\hline $1 b$ & iii & 400 & 68 & 72.33 & 3.616 & 0.90 & 53 & 47 & 1.92 \\
\hline \multicolumn{6}{|c|}{$1 \mathrm{~b}$ Mean } & 0.96 & & & 2.04 \\
\hline & 0.11 \\
\hline & & & & & & & & & 1.96 \\
\hline \multicolumn{9}{|c|}{ Recipe 1 Mean } & 0.11 \\
\hline
\end{tabular}

\begin{tabular}{|c|c|c|c|c|c|c|c|c|c|}
\hline Reactor & Sample & $\begin{array}{c}\text { Sample Size } \\
\text { (mg) }\end{array}$ & $\begin{array}{c}\text { \# of } \\
\text { squares }\end{array}$ & $\mathrm{mg} \mathrm{N} / \mathrm{L}$ & $\begin{array}{c}\text { mg of Nin } \\
\text { sample }\end{array}$ & $\% \mathrm{~N}(w b)$ & $\%$ MC & $\%$ TS & $\% N(d b)$ \\
\hline $2 \mathrm{a}$ & 1 & 400 & 56 & 59.56 & 2.978 & 0.74 & 69 & 31 & 2.40 \\
\hline $2 a$ & ii & 400 & 54 & 57.43 & 2.872 & 0.72 & 69 & 31 & 2.32 \\
\hline $2 \mathrm{a}$ & iii & 400 & 60 & 63.82 & 3.191 & 0.80 & 69 & 31 & 2.57 \\
\hline \multicolumn{6}{|l|}{ 2a Mean } & 0.75 & & & 2.43 \\
\hline \multicolumn{6}{|c|}{ 2a St. Dev. } & 0.04 & & & 0.13 \\
\hline $2 \mathrm{~b}$ & $i$ & 400 & 50 & 53.18 & 2.659 & 0.66 & 71 & 29 & 2.29 \\
\hline $2 b$ & ii & 400 & 64 & 68.07 & 3.404 & 0.85 & 71 & 29 & 2.93 \\
\hline $2 b$ & iii & 400 & 63 & 67.01 & 3.350 & 0.84 & 71 & 29 & 2.89 \\
\hline \multicolumn{6}{|l|}{ 2b Mean } & 0.78 & & & 2.70 \\
\hline & & & & & & 0.10 & & & 0.36 \\
\hline \multicolumn{9}{|c|}{ Recipe 2 Mean } & 2.57 \\
\hline \multicolumn{9}{|c|}{ Recipe 2 St. Dev. (between 2a and 2b) } & 0.19 \\
\hline
\end{tabular}

\begin{tabular}{|c|c|c|c|c|c|c|c|c|c|}
\hline Reactor & Sample & $\begin{array}{c}\text { Sample Size } \\
\text { (mg) }\end{array}$ & $\begin{array}{c}\text { \# of } \\
\text { squares }\end{array}$ & $\mathrm{mg} \mathrm{N} / \mathrm{L}$ & $\begin{array}{c}\text { mg of } \mathrm{Nin} \\
\text { sample }\end{array}$ & $\% N(w b)$ & $\% \mathrm{MC}$ & $\%$ TS & $\% N(d b)$ \\
\hline $3 a$ & $i$ & 400 & 78 & 82.96 & 4.148 & 1.04 & 39 & 61 & 1.70 \\
\hline $3 a$ & ii & 400 & 84 & 89.34 & 4.467 & 1.12 & 39 & 61 & 1.83 \\
\hline $3 a$ & iii & 400 & 78 & 82.96 & 4.148 & 1.04 & 39 & 61 & 1.70 \\
\hline \multicolumn{6}{|l|}{ 3a Mean } & 1.06 & & & 1.74 \\
\hline \multicolumn{6}{|c|}{ 3a St. Dev. } & 0.05 & & & 0.08 \\
\hline $3 \mathrm{~b}$ & $i$ & 400 & 83 & 88.28 & 4.414 & 1.10 & 37 & 63 & 1.75 \\
\hline $3 b$ & ii & 400 & 82 & 87.22 & 4.361 & 1.09 & 37 & 63 & 1.73 \\
\hline $3 b$ & iii & 400 & 85 & 90.41 & 4.520 & 1.13 & 37 & 63 & 1.79 \\
\hline \multicolumn{6}{|l|}{ 3b Mean } & 1.11 & & & 1.76 \\
\hline $3 b$ St. De & & & & & & 0.02 & & & 0.03 \\
\hline & 1.75 \\
\hline & \multicolumn{8}{|c|}{ Recipe 3 St. Dev. (between $3 a$ and 3b) } & 0.01 \\
\hline
\end{tabular}


Phase 1 - Final TKN Analysis

\begin{tabular}{|c|c|c|c|c|c|c|c|c|c|}
\hline Reactor & Sample & $\begin{array}{c}\text { Sample Size } \\
\text { (mg) }\end{array}$ & $\begin{array}{c}\text { \# of } \\
\text { squares }\end{array}$ & $\mathrm{mg} \mathrm{N} / \mathrm{L}$ & $\begin{array}{c}\text { mg of } \mathrm{N} \text { in } \\
\text { sample }\end{array}$ & $\% \mathrm{~N}(w b)$ & $\% \mathrm{MC}$ & $\%$ TS & $\% \mathrm{~N}(\mathrm{db})$ \\
\hline $4 a$ & $i$ & 400 & 73 & 77.64 & 3.882 & 0.97 & 51 & 49 & 1.98 \\
\hline $4 a$ & iI & 400 & 77 & 81.90 & 4.095 & 1.02 & 51 & 49 & 2.09 \\
\hline $4 \mathrm{a}$ & iii & 400 & 74 & 78.71 & 3.935 & 0.98 & 51 & 49 & 2.01 \\
\hline \multicolumn{6}{|c|}{ 4a Mean } & 0.99 & & & 2.03 \\
\hline \multicolumn{6}{|c|}{ 4a St. Dev. } & 0.03 & & & 0.06 \\
\hline $4 \mathrm{~b}$ & $i$ & 400 & 72 & 76.58 & 3.829 & 0.96 & 53 & 47 & 2.04 \\
\hline $4 \mathrm{~b}$ & ii & 400 & 71 & 75.52 & 3.776 & 0.94 & 53 & 47 & 2.01 \\
\hline $4 b$ & iii & 400 & 74 & 78.71 & 3.935 & 0.98 & 53 & 47 & 2.09 \\
\hline \multicolumn{6}{|c|}{$4 \mathrm{~b}$ Mean } & 0.96 & & & 2.05 \\
\hline \multicolumn{6}{|c|}{$4 b$ St. Dev. } & 0.02 & & & 0.04 \\
\hline \multicolumn{9}{|c|}{ Recipe 4 Mean } & 2.04 \\
\hline \multicolumn{9}{|c|}{ Recipe 4 St. Dev. (between $4 a$ and $4 b$ ) } & 0.01 \\
\hline
\end{tabular}

\begin{tabular}{|c|c|c|c|c|c|c|c|c|c|}
\hline Reactor & Sample & $\begin{array}{l}\text { Sample Size } \\
(\mathrm{mg})\end{array}$ & $\begin{array}{c}\text { \# of } \\
\text { squares }\end{array}$ & $\mathrm{mg} \mathrm{N} / \mathrm{L}$ & $\begin{array}{c}\text { mg of } N \text { in } \\
\text { sample }\end{array}$ & $\% N(w b)$ & $\% \mathrm{MC}$ & $\%$ TS & $\% \mathrm{~N}(\mathrm{db})$ \\
\hline $5 a$ & $\mathrm{i}$ & 400 & 62 & 65.94 & 3.297 & 0.82 & 60 & 40 & 2.06 \\
\hline $5 a$ & ii & 400 & 65 & 69.13 & 3.457 & 0.86 & 60 & 40 & 2.16 \\
\hline $5 a$ & iii & 400 & 64 & 68.07 & 3.404 & 0.85 & 60 & 40 & 2.13 \\
\hline \multicolumn{6}{|l|}{ 5a Mean } & 0.85 & & & 2.12 \\
\hline \multicolumn{6}{|c|}{ 5a St. Dev. } & 0.02 & & & 0.05 \\
\hline $5 b$ & $i$ & 400 & 69 & 73.39 & 3.669 & 0.92 & 61 & 39 & 2.35 \\
\hline $5 b$ & ii & 400 & 67 & 71.26 & 3.563 & 0.89 & 61 & 39 & 2.28 \\
\hline $5 b$ & iii & 400 & 67 & 71.26 & 3.563 & 0.89 & 61 & 39 & 2.28 \\
\hline \multicolumn{6}{|c|}{5 b Mean } & 0.90 & & & 2.31 \\
\hline $5 \mathrm{~b}$ St. De & & & & & & 0.02 & & & 0.04 \\
\hline Recipe 5 & an & & & & & & & & 2.21 \\
\hline \multicolumn{9}{|c|}{ Recipe 5 St. Dev. (between $5 a$ and 5b) } & 0.13 \\
\hline
\end{tabular}

\begin{tabular}{|c|c|c|c|c|c|c|c|c|c|}
\hline Reactor & Sample & $\begin{array}{c}\text { Sample Size } \\
(\mathrm{mg})\end{array}$ & $\begin{array}{c}\text { \# of } \\
\text { squares }\end{array}$ & $\mathrm{mg} \mathrm{N} / \mathrm{L}$ & $\begin{array}{c}\text { mg of } \mathrm{N} \text { in } \\
\text { sample }\end{array}$ & $\% N(w b)$ & $\% \mathrm{MC}$ & $\%$ TS & $\% \mathrm{~N}(\mathrm{db})$ \\
\hline $6 a$ & $\mathrm{i}$ & 400 & 56 & 59.56 & 2.978 & 0.74 & 71 & 29 & 2.57 \\
\hline $6 a$ & ii & 400 & 54 & 57.43 & 2.872 & 0.72 & 71 & 29 & 2.48 \\
\hline $6 a$ & iii & 400 & 54 & 57.43 & 2.872 & 0.72 & 71 & 29 & 2.48 \\
\hline \multicolumn{6}{|c|}{ 6a Mean } & 0.73 & & & 2.51 \\
\hline \multicolumn{6}{|c|}{ 6a St. Dev. } & 0.02 & & & 0.05 \\
\hline $6 \mathrm{~b}$ & $i$ & 400 & 53 & 56.37 & 2.819 & 0.70 & 72 & 28 & 2.52 \\
\hline $6 \mathrm{~b}$ & ii & 400 & 54 & 57.43 & 2.872 & 0.72 & 72 & 28 & 2.56 \\
\hline $6 \mathrm{~b}$ & iil & 400 & 56 & 59.56 & 2.978 & 0.74 & 72 & 28 & 2.66 \\
\hline \multicolumn{6}{|l|}{ 6b Mean } & 0.72 & & & 2.58 \\
\hline \multicolumn{6}{|c|}{ 6b St. Dev. } & 0.02 & & & 0.07 \\
\hline \multirow{2}{*}{\multicolumn{9}{|c|}{ Recipe 6 Mean }} & 2.54 \\
\hline \multicolumn{6}{|c|}{ Recipe 6 St. Dev. (between 6a and 6b) } & & & & 0.05 \\
\hline
\end{tabular}

\begin{tabular}{|c|c|c|c|c|c|c|c|c|c|}
\hline Reactor & Sample & $\begin{array}{c}\text { Sample Size } \\
(\mathrm{mg})\end{array}$ & $\begin{array}{c}\text { \# of } \\
\text { squares }\end{array}$ & $\mathrm{mg} \mathrm{N} / \mathrm{L}$ & $\begin{array}{c}\mathrm{mg} \text { of } \mathrm{N} \text { in } \\
\text { sample }\end{array}$ & $\% N(w b)$ & $\% \mathrm{MC}$ & $\%$ TS & $\% \mathrm{~N}(\mathrm{db})$ \\
\hline $7 a$ & $\mathrm{i}$ & 300 & 55 & 58.50 & 2.925 & 0.97 & 61 & 39 & 2.50 \\
\hline $7 a$ & ii & 300 & 56 & 59.56 & 2.978 & 0.99 & 61 & 39 & 2.55 \\
\hline $7 a$ & iii & 300 & 56 & 59.56 & 2.978 & 0.99 & 61 & 39 & 2.55 \\
\hline \multicolumn{6}{|c|}{ 7a Mean } & 0.99 & & & 2.53 \\
\hline \multicolumn{6}{|c|}{ 7a St. Dev. } & 0.01 & & & 0.03 \\
\hline $7 \mathrm{~b}$ & $i$ & 300 & 53 & 56.37 & 2.819 & 0.94 & 60 & 40 & 2.35 \\
\hline $7 \mathrm{~b}$ & ii & 300 & 58 & 61.69 & 3.084 & 1.03 & 60 & 40 & 2.57 \\
\hline $7 \mathrm{~b}$ & iii & 300 & 55 & 58.50 & 2.925 & 0.97 & 60 & 40 & 2.44 \\
\hline \multicolumn{6}{|c|}{$7 \mathrm{~b}$ Mean } & 0.98 & & & 2.45 \\
\hline \multicolumn{6}{|c|}{ 7b St. Dev. } & 0.04 & & & 0.11 \\
\hline \multicolumn{9}{|c|}{ Recipe 7 Mean } & 2.49 \\
\hline \multicolumn{9}{|c|}{ Recipe 7 St. Dev. (between 7a and 7b) } & 0.06 \\
\hline
\end{tabular}

Notes:

1) Analysis completed on November 16, 2001.

2) \%MC and \%TS values from final Phase I solids analysis. 


\section{Phase 1 - Feedstock Characterization}

\section{Wet Bulk Density - Straw}

Tested June 21, 2001

Used a 2 US gallon container to measure the wet bulk density 2 US gal $=0.007571 \mathrm{~m}^{3}$

2 US gal $=\quad 7.571 \mathrm{~L}$

\begin{tabular}{|c|c|c|c|}
\hline Sample & Tare $(\mathrm{kg})$ & Tare + material $(\mathrm{kg})$ & Bulk Density $\left(\mathrm{kg} / \mathrm{m}^{3}\right)$ \\
\hline Bale 1 & 0.4 & 1.22 & 108.31 \\
\hline Bale 2 & 0.4 & 0.75 & 46.23 \\
\hline Bale 3 & 0.4 & 0.79 & 51.51 \\
\hline Bale 4 & 0.4 & 0.91 & 67.36 \\
\hline Bale 5 & 0.4 & 1.69 & 170.39 \\
\hline \multicolumn{3}{|c|}{$\begin{array}{r}\text { Mean }= \\
\text { Std. Dev. }= \\
\text { Literature value (Rynk, 1992) }=\end{array}$} & $\begin{array}{c}88.8 \\
51.7 \\
1\end{array}$ \\
\hline
\end{tabular}

Notes:

1) All samples collected on June 18, 2001. 
Phase 1 - Example of Moisture Content Adjustment Calculation Sheet

\begin{tabular}{|c|c|c|}
\hline Initial MC $=$ & 49 & $\%$ \\
\hline Target $M C=$ & 57 & $\%$ \\
\hline$M C=$ & $($ wet - dry)/wet & \\
\hline original mass of sample (wet) $=$ & 106.58 & $\mathrm{gg}$ \\
\hline calculated dry mass $=$ & 54.36 & $g$ \\
\hline target wet mass (calculated) $=$ & 126.41 & $\mathrm{~g}$ \\
\hline Water addition needed $=$ & 19.83 & g or $\mathrm{mL}$ \\
\hline
\end{tabular}

Dry Mass Calculation:

dry mass $=$ wet mass $-(\% \mathrm{MC} / 100)^{*}$ wet mass

Target Wet Mass Calculation:

target wet mass = calculated dry mass $/(1-$ target $\% \mathrm{MC} / 100)$

Water Addition Needed Calculation:

Water needed $=$ target wet mass - original wet mass

\begin{tabular}{|c|c|c|}
\hline Initial $M C=$ & 55 & $1 \%$ \\
\hline Desired MC $=$ & 57 & $\%$ \\
\hline$M C=$ & (wet - dry)/wet & \\
\hline original mass of sample (wet) $=$ & 120.91 & \\
\hline calculated dry mass $=$ & 54.41 & $\frac{g}{g}$ \\
\hline desired wet mass (calculated) $=$ & 126.53 & $g$ \\
\hline Water addition needed $=$ & 5.62 & $\mathrm{~g}$ or $\mathrm{mL}$ \\
\hline
\end{tabular}

\section{This number varies with each moisture test}

$163.65 \mathrm{~g}=$ tare weight

$400.08 \mathrm{~g}=$ mass at start up (tare+material)

$236.43 \mathrm{~g}=$ mass at start up (material only)

$209.57 \mathrm{~g}=$ mass at start up (aiter sampling, material only)

$373.22 \mathrm{~g}=$ mass at start up (after sampling, tare + material)

$290.06 \mathrm{~g}=$ proper mass after MC adjustment (tare + material)

This number varies with each moisture test

This number varies with each moisture test

$163.50 \mathrm{~g}=$ tare weight

$400.15 \mathrm{~g}=$ mass at start up (tare+material)

$236.65 \mathrm{~g}=$ mass at start up (material only)

$209.37 \mathrm{~g}=$ mass at start up (after sampling, material only)

$372.87 \mathrm{~g}=$ mass at start up (after sampling, tare + material)

$290.03 \mathrm{~g}=$ proper mass after MC adjustment (tare + material) 
Phase 1 - Moisture Content Measurements

\begin{tabular}{|c|c|c|c|c|c|c|c|c|c|c|c|c|c|c|c|c|c|c|c|c|c|c|c|c|c|c|c|c|}
\hline \multirow{2}{*}{ Recipe Description } & & Day & Day & Day & Day & Day & Day & Day & Day & Day & Day & Day & Day & Day & Day & Day & $\begin{array}{l}\text { Day } \\
53\end{array}$ & $\begin{array}{c}\text { Day } \\
55\end{array}$ & $\begin{array}{l}\text { Day } \\
57\end{array}$ & $\begin{array}{l}\text { Day } \\
59\end{array}$ & $\begin{array}{c}\text { Day } \\
61\end{array}$ & $\begin{array}{l}\text { Day } \\
63\end{array}$ & Mean & $\begin{array}{l}\text { St. } \\
\text { Dev. }\end{array}$ & \multicolumn{2}{|c|}{ Excluding Day 28} & \multicolumn{2}{|c|}{ After Day 47} \\
\hline & $\#$ & 8 & 13 & 17 & 21 & & & & & & & & & & & & & & & & & & & & Mean & St.Dev. & Mean & St.Dev. \\
\hline Straw & $\begin{array}{l}1 \mathrm{a} \\
\end{array}$ & 49 & 49 & $\overline{51}$ & 54 & 28 & $\overline{14}$ & 40 & 52 & 52 & 50 & 49 & 22 & 11 & 46 & 49 & 42 & 47 & 51 & 46 & 55 & 49 & 43 & 13 & 44 & 12 & 48 & 4 \\
\hline$(M G=57 \%)$ & $1 \mathrm{ab}$ & 38 & $\frac{45}{54}$ & 50 & $\frac{37}{9}$ & 47 & 50 & 39 & 51 & 45 & 57 & 54 & 24 & 24 & 49 & 44 & 44 & 45 & 50 & 52 & 54 & 55 & 45 & 12 & 44 & 12 & 48 & 4 \\
\hline$\frac{1}{\text { Straw }}$ & $2 a$ & 61 & $\frac{64}{60}$ & 60 & $\frac{5}{68}$ & 63 & 28 & 48 & 65 & 67 & 70 & 44 & 38 & 34 & 60 & 63 & 51 & 66 & 67 & 67 & 70 & 64 & 58 & 12 & 59 & 11 & 63 & 6 \\
\hline$(M C=70 \%)$ & $2 \mathrm{~b}$ & 57 & 70 & 63 & 65 & 70 & 10 & 70 & 56 & 62 & 70 & 52 & 44 & 45 & 67 & 41 & 62 & 67 & 69 & 65 & 70 & 66 & 59 & 14 & 61 & 10 & 63 & 10 \\
\hline Straw + Unders & $3 a$ & 20 & 29 & 29 & 10 & 6 & 5 & 24 & 35 & 34 & 37 & 11 & 11 & 15 & 31 & 29 & 34 & 35 & 37 & 33 & 33 & 38 & 26 & 11 & 26 & 10 & 33 & 3 \\
\hline$=40 \%$ & $3 b$ & 27 & 37 & 6 & 13 & 6 & 4 & 32 & 34 & 24 & 38 & 26 & 18 & 6 & 29 & 22 & 18 & 33 & 39 & 37 & 38 & 40 & 25 & 12 & 25 & 11 & 31 & 8 \\
\hline Straw + Unders & $4 a$ & 38 & 55 & 48 & 50 & 48 & 5 & 34 & 51 & 53 & 49 & 40 & 41 & 6 & 42 & 37 & 48 & 54 & 52 & 52 & 53 & 46 & 43 & 14 & 45 & 11 & 48 & 6 \\
\hline$=55 \%$ & $4 \mathrm{~b}$ & 45 & 55 & 9 & 53 & 10 & 36 & 22 & 48 & 53 & 50 & 52 & 48 & 46 & 48 & 54 & 51 & 54 & 51 & 45 & 51 & 55 & 45 & 14 & 44 & 14 & 51 & 3 \\
\hline Straw + Unders & $5 a$ & 56 & 62.5 & 61 & 60 & 27 & 6 & 41 & 54 & 55 & 61 & $\frac{3 c}{37}$ & 31 & 22 & 47 & 16 & 40 & 60 & 57 & 61 & 61 & 62 & 47 & 17 & 48 & 15 & 49 & 17 \\
\hline$=62.5 \%)$ & $5 b$ & 57 & 62.5 & 16 & 56 & 56 & 5 & 54 & 45 & 59 & 61 & 44 & 25 & 28 & 48 & 47 & 55 & 60 & 62 & 62 & 56 & 59 & 48 & 16 & 50 & 14 & 56 & 6 \\
\hline Straw + Unders & $6 a$ & 60 & 70 & 69 & 63 & 60 & 5 & 53 & 65 & 63 & 69 & 66 & 68 & 65 & 39 & 48 & 46 & 67 & 66 & 69 & 67 & 68 & 59 & 15 & 62 & 9 & 57 & 13 \\
\hline$=70 \%)$ & $6 \mathrm{~b}$ & 68 & 69 & 69 & 46 & 57 & 5 & 42 & 67 & 63 & 68 & 66 & 64 & 64 & 27 & 21 & 57 & 67 & 68 & 62 & 67 & 67 & 56 & 18 & 59 & 14 & 53 & 20 \\
\hline Straw+Unders+Lagoon & $7 a$ & 56 & 60 & 62.5 & 42 & 32 & 9 & 29 & 58 & 61 & 60 & 54 & 56 & 32 & 57 & 54 & 33 & 59 & 53 & 58 & 62 & 60 & 50 & 15 & 52 & 12 & 54 & 10 \\
\hline$M C=62.5 \%$ & $7 \mathrm{~b}$ & 58 & 47 & 27 & 57 & 42 & 32 & 42 & 58 & 62 & 60 & 58 & 24 & 9 & 55 & 45 & 30 & 55 & 58 & 58 & 59 & 61 & 47 & 15 & 48 & 15 & 51 & 11 \\
\hline
\end{tabular}

Notes:

1) All moisture contents are indicated in $\%$

1) All moisture contents are indicated in \%.

3) Moisture content on Day 1 as per recipe description. 
Phase 1 - Moisture Content Losses

\begin{tabular}{|c|c|c|c|c|c|c|c|c|c|c|c|c|c|c|c|c|c|c|c|c|c|c|c|c|c|c|c|c|}
\hline Bucket Description & $\#$ & $\begin{array}{c}\text { Day } \\
8\end{array}$ & $\begin{array}{c}\text { Day } \\
13\end{array}$ & $\begin{array}{c}\text { Day } \\
17\end{array}$ & $\begin{array}{c}\text { Day } \\
21\end{array}$ & $\begin{array}{c}\text { Day } \\
24\end{array}$ & $\begin{array}{c}\text { Day } \\
28\end{array}$ & $\begin{array}{c}\text { Day } \\
30\end{array}$ & $\begin{array}{c}\text { Day } \\
33\end{array}$ & $\begin{array}{c}\text { Day } \\
36\end{array}$ & $\begin{array}{c}\text { Day } \\
39\end{array}$ & \begin{tabular}{|c|} 
Day \\
42 \\
\end{tabular} & \begin{tabular}{|c|} 
Day \\
44
\end{tabular} & $\begin{array}{l}\text { Day } \\
47\end{array}$ & \begin{tabular}{|c|} 
Day \\
49
\end{tabular} & $\begin{array}{c}\text { Day } \\
51\end{array}$ & \begin{tabular}{|c|} 
Day \\
53 \\
\end{tabular} & \begin{tabular}{|c|} 
Day \\
55
\end{tabular} & \begin{tabular}{|c|} 
Day \\
57 \\
\end{tabular} & $\begin{array}{c}\text { Day } \\
59\end{array}$ & \begin{tabular}{|c|} 
Day \\
61
\end{tabular} & \begin{tabular}{|c|} 
Day \\
63
\end{tabular} & Mean & $\begin{array}{c}\text { St. } \\
\text { Dev. }\end{array}$ & \multicolumn{2}{|c|}{ Excluding Day 28} & \multicolumn{2}{|c|}{ After Day 47} \\
\hline & & & & & & & & & & & & & & & & & & & & & & & & & Mean & St.Dev. & Mean & St.Dev. \\
\hline Straw & $1 \mathrm{a}$ & 8 & 8 & 6 & 3 & 29 & 43 & 17 & 5 & 5 & 7 & 8 & 35 & 46 & 11 & 8 & 15 & 10 & 6 & 11 & 2 & 8 & 14 & 13 & 13 & 12 & 9 & 4 \\
\hline$(M C=57 \%)$ & $1 \mathrm{~b}$ & 19 & 3 & .7 & 48 & 10 & 7 & 18 & 6 & 12 & 0 & 3 & 33 & 33 & 8 & 13 & 13 & 12 & 7 & 5 & 3 & 2 & 12 & 12 & 13 & 12 & $\frac{9}{7}$ & 4 \\
\hline Straw & $2 a$ & 9 & 10 & 10 & 2 & 7 & 42 & 22 & 5 & 3 & 0 & 26 & 32 & 36 & 10 & 7 & 19 & 4 & 3 & 3 & 0 & 6 & 12 & 13 & 11 & 11 & 7 & 6 \\
\hline$(M C=70 \%)$ & $2 b$ & 13 & 0 & 7 & 5 & 0 & 60 & 0 & 14 & 8 & 0 & 18 & 26 & 25 & 3 & 29 & 8 & 3 & 1 & 5 & 0 & 4 & 11 & 15 & 9 & 10 & 7 & 10 \\
\hline Straw + Unders & $3 a$ & 20 & 11 & 11 & 30 & 34 & 35 & 16 & 5 & 6 & 3 & 29 & 29 & 25 & 9 & 11 & 6 & 5 & 3 & 7 & 7 & 2 & 14 & 11 & 14 & 10 & 7 & 3 \\
\hline$=40 \%$ & $3 b$ & 13 & 3 & 34 & 27 & 34 & 36 & 8 & 6 & 16 & 2 & 14 & 22 & 34 & 11 & 18 & 22 & 7 & 1 & 3 & 2 & 0 & 15 & 12 & 15 & 11 & 9 & 8 \\
\hline Straw + Unders & $4 a$ & 17 & 0 & 7 & 5 & 7 & 50 & 21 & 4 & 2 & 6 & 15 & 14 & 49 & 13 & 18 & 7 & 1 & 3 & 3 & 2 & 9 & 12 & 14 & 10 & 11 & 7 & 6 \\
\hline$=55 \%$ & $4 b$ & 10 & 0 & 46 & 2 & 45 & 19 & 33 & 7 & 2 & 5 & 3 & 7 & 9 & 7 & 1 & 4 & 1 & 4 & 10 & 4 & 0 & 10 & 14 & 11 & 14 & 4 & 3 \\
\hline Straw + Unders & $5 a$ & 6.5 & 0 & 1.5 & 2.5 & 35.5 & 56.5 & 21.5 & 8.5 & 7.5 & 1.5 & 25.5 & 31.5 & 40.5 & 15.5 & 46.5 & 22.5 & 2.5 & 5.5 & 1.5 & 1.5 & 0.5 & 16 & 17 & 15 & 15 & 14 & 17 \\
\hline$=62.5 \%$ & $5 b$ & 5.5 & 0 & 46.5 & 6.5 & 6.5 & 57.5 & 8.5 & 17.5 & 3.5 & 1.5 & 18.5 & 37.5 & 34.5 & 14.5 & 15.5 & 7.5 & 2.5 & 0.5 & 0.5 & 6.5 & 3.5 & 14 & 17 & 12 & 14 & 7 & 6 \\
\hline Straw + Unders & $6 a$ & 10 & 0 & 1 & 7 & 10 & 65 & 17 & 5 & 7 & 1 & 4 & 2 & 5 & 31 & 22 & 24 & 3 & 4 & 1 & 3 & 2 & 11 & 15 & 8 & 9 & 13 & 13 \\
\hline$=70 \%$ & $6 \mathrm{~b}$ & 2 & 1 & 1 & 24 & 13 & 65 & 28 & 3 & 7 & 2 & 4 & 6 & 6 & 43 & 49 & 13 & 3 & 2 & 8 & 3 & 3 & 14 & 18 & 11 & 14 & 17 & 20 \\
\hline Straw+Unders+Lagoon & $7 \mathrm{a}$ & 6.5 & 2.5 & 0 & 20.5 & 30.5 & 53.5 & 33.5 & 4.5 & 1.5 & 2.5 & 8.5 & 6.5 & 30.5 & 5.5 & 8.5 & 29.5 & 3.5 & 9.5 & 4.5 & 0.5 & 25 & 13 & 15 & 11 & 12 & 9 & 10 \\
\hline$(M C=62.5 \%)$ & $7 \mathrm{~b}$ & 4.5 & 15.5 & 35.5 & 5.5 & 20.5 & 30.5 & 20.5 & 4.5 & 0.5 & 2.5 & 4.5 & 38.5 & 53.5 & 7.5 & 17.5 & 32.5 & 7.5 & 4.5 & 4.5 & 3.5 & 1.5 & 15 & 15 & 15 & 15 & 11 & 11 \\
\hline & & 10 & 4 & \begin{tabular}{|l|}
15 \\
17
\end{tabular} & $\frac{13}{14}$ & \begin{tabular}{|l|}
20 \\
14 \\
\end{tabular} & \begin{tabular}{|l|}
44 \\
17
\end{tabular} & \begin{tabular}{|c|}
19 \\
9
\end{tabular} & $\frac{7}{4}$ & $\frac{6}{4}$ & $\frac{2}{2}$ & $\frac{13}{9}$ & $-\frac{23}{13}$ & $\begin{array}{l}31 \\
15\end{array}$ & $\frac{14}{11}$ & $\frac{19}{14}$ & \begin{tabular}{|l|}
16 \\
9
\end{tabular} & $\frac{5}{3}$ & $\frac{4}{3}$ & $\frac{5}{3}$ & $\frac{3}{2}$ & $\frac{3}{3}$ & $\frac{13}{2}$ & & $\frac{12}{2}$ & & $\frac{9}{3}$ & \\
\hline
\end{tabular}

Notes:

1) All moisture losses are indicated in \%.

2) All moisture contents measured before moisture content adjustment $\left(\mathrm{H}_{2} \mathrm{O}\right.$ addrition).

3) Moisture content on Day 1 as per recipe description. 
Phase 1 - Odour Observation Summary

\begin{tabular}{|c|c|c|c|c|c|c|c|c|c|c|}
\hline Reactor & Day 1 & Day 17 & Day 21 & Day 24 & Day 28 & Day 36 & Day 49 & Day 55 & Day 63 & Average \\
\hline $1 \mathrm{a}$ & 1 & 1 & 1.5 & 1 & 1 & 2 & 1 & 0.5 & 0.5 & 1.1 \\
\hline $1 \mathrm{~b}$ & 1 & 1 & 0.5 & 2 & 1 & 1 & 1 & 0.5 & 0.5 & 0.9 \\
\hline $2 \mathrm{a}$ & 1 & 1 & 1 & 1.5 & 1 & 1 & 1 & 1 & 0.5 & 1.0 \\
\hline $2 \mathrm{~b}$ & 1 & 1 & 1 & 1.5 & 0.5 & 1.5 & 1 & 1 & 0.5 & 1.0 \\
\hline $3 \mathrm{a}$ & 1 & 2 & 1.5 & 0.5 & 0.5 & 1.5 & 1 & 1 & 0.5 & 1.1 \\
\hline $3 \mathrm{~b}$ & 1 & 2 & 1.5 & 0.5 & 0.5 & 1 & 1 & 1 & 0.5 & 1.0 \\
\hline $4 \mathrm{a}$ & 1 & 1.5 & 1 & 1 & 1 & 1 & 0.5 & 1 & 0.5 & 0.9 \\
\hline $4 \mathrm{~b}$ & 1 & 1 & 1 & 1 & 1 & 1 & 0.5 & 1 & 0.5 & 0.9 \\
\hline $5 \mathrm{a}$ & 1 & 1 & 1 & 1 & 1 & 0.5 & 1 & 1 & 0.5 & 0.9 \\
\hline $5 \mathrm{~b}$ & 1 & 1 & 1 & 1.5 & 1 & 0.5 & 1 & 1 & 0.5 & 0.9 \\
\hline $6 \mathrm{a}$ & 1 & 1 & 1 & 1 & 1 & 0.5 & 1 & 1 & 0.5 & 0.9 \\
\hline $6 \mathrm{~b}$ & 1 & 1 & 1 & 1 & 1 & 0.5 & 1 & 1 & 0.5 & 0.9 \\
\hline $7 \mathrm{a}$ & 3 & 1.5 & 1 & 1 & 1 & 1 & 0.5 & 0.5 & 0.5 & 1.1 \\
\hline $7 \mathrm{~b}$ & 3 & 1.5 & 1.5 & 1 & 2 & 1 & 0.5 & 0.5 & 0.5 & 1.3 \\
\hline
\end{tabular}

Scale:

Agreeable Odour Disagreeable Odour

1

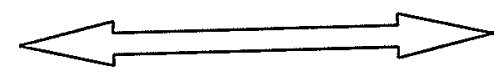


Phase 1 - Texture Comments Summary

Reactor 1a - Straw Only (57\% MC)

\begin{tabular}{|c|l|}
\hline Day & Comments \\
\hline 1 & partially coarse straw \\
\hline 17 & dry, individual straw particles become smaller with mixing action \\
\hline 21 & wetter than 1b; soft, fluffy particles \\
\hline 24 & dry; smaller straw particles \\
\hline 28 & dry \\
\hline 36 & light, soft, not much degradation of straw particles \\
\hline 49 & damp; softer than straw at startup \\
\hline 55 & particle like \\
\hline 63 & evident straw particles; not crunchy \\
\hline
\end{tabular}

Reactor 1 b - Straw Only (57\% MC)

\begin{tabular}{|c|l|}
\hline Day & lomments \\
\hline 1 & partially coarse straw \\
\hline 17 & dry, individual straw particles become smaller with mixing action \\
\hline 21 & dried out and dusty; brittle straw particles \\
\hline 24 & dry; smaller straw particles \\
\hline 28 & less dry than 1a, softer than 1a \\
\hline 36 & light, soft, not much degradation of straw particles \\
\hline 49 & damp; softer than straw at startup \\
\hline 55 & particle like \\
\hline 63 & evident straw particles; not crunchy \\
\hline
\end{tabular}

Notes:

1) Observations made before moisture content adjustments $\left(\mathrm{H}_{2} \mathrm{O}\right.$ addition).

2) Smaller particles size may be due to brittleness of dry reactor contents. 
Phase 1 - Texture Comments Summary

Reactor $2 a$ - Straw Only (70\% MC)

\begin{tabular}{|c|l|}
\hline Day & Comments \\
\hline 1 & wetter straw, less coarse \\
\hline 17 & $\begin{array}{l}\text { less fagile particles than } 1 \mathrm{a}, 1 \mathrm{~b} ; \text { a lottle larger in size than } 1 \mathrm{a}, \mathrm{b} \text {; wet feeling; crusty on } \\
\text { outsides expecially on top; wet on inside (core) }\end{array}$ \\
\hline 21 & crunchy on top before mixing; wet/soft feel \\
\hline 24 & wet; still longer straw particles \\
\hline 28 & very dry particles \\
\hline 36 & wet, somewhat mushy \\
\hline 49 & soft; drier particles get very hard; some clumps formed \\
\hline 55 & very wet, sticky, clumpy \\
\hline 63 & wet; more visible particles than in $6 a, b ;$ top layer of straw is a little crunchy/hard \\
\hline
\end{tabular}

Reactor $2 \mathrm{~b}$ - Straw Only (70\% MC)

\begin{tabular}{|c|l|}
\hline Day & Comments \\
\hline 1 & wetter straw, less coarse \\
\hline 17 & $\begin{array}{l}\text { less fagile particles than 1a, } 1 \mathrm{~b} ; \text { a lottle larger in size than } 1 \mathrm{a}, 1 \mathrm{~b} ; \text { wet feeling; crusty on } \\
\text { outsides expecially on top; wet on inside (core) }\end{array}$ \\
\hline 21 & crunchy on top before mixing; wet/soft feel \\
\hline 24 & very wet; initially crunchy on top; still long particles of straw \\
\hline 28 & very dry particles; agglomerated chunks \\
\hline 36 & wet, somewhat mushy \\
\hline 49 & soft; drier particles get very hard; some clumps formed \\
\hline 55 & very wet, sticky, clumpy \\
\hline 63 & wet; more visible particles than in 6a,b; top layer of straw is a little crunchy/hard \\
\hline
\end{tabular}

Notes:

1) Observations made before moisture content adjustments ( $\mathrm{H}_{2} \mathrm{O}$ addition).

2) Smaller particles size may be due to brittleness of dry reactor contents. 
Phase 1 - Texture Comments Summary

Reactor $3 \mathrm{a}$ - Straw and Unders (40\% MC)

\begin{tabular}{|c|l|}
\hline Day & Comments \\
\hline 1 & dry, dusty mixture, fuller body (unders fill spaces between straw particles) \\
\hline 17 & $\begin{array}{l}\text { unders are separate; dusty unders on bottom; some under in clumps on bottom (baked into } \\
\text { corners) }\end{array}$ \\
\hline 21 & little combining of two materials; brittle straw particles \\
\hline 24 & $\begin{array}{l}\text { some of unders stuck to straw particles (cover straw in coating) but a lot of straw particles } \\
\text { uncoated and crunchy; some unders clumped together; still dry }\end{array}$ \\
\hline 28 & light; fluffy; still separated unders/straw \\
\hline 36 & soft, fluffy, some instances of under clumping; very little degradation \\
\hline 49 & $\begin{array}{l}\text { still separation of materials; dusty at times; some clumpls of unders; center contains most } \\
\text { of moisture }\end{array}$ \\
\hline 55 & still a mixture; light, dusty \\
\hline 63 & separate straw/unders; damp unders \\
\hline
\end{tabular}

Reactor $3 \mathrm{~b}$ - Straw and Unders ( $40 \%$ MC)

\begin{tabular}{|c|l|}
\hline Day & lomments \\
\hline 1 & dry, dusty mixture, fuller body (unders fill spaces between straw particles) \\
\hline 17 & $\begin{array}{l}\text { unders are separate; dusty unders on bottom; some under in clumps on bottom (baked into } \\
\text { corners) }\end{array}$ \\
\hline 21 & little combining of two materials; brittle straw particles \\
\hline 24 & $\begin{array}{l}\text { lome of unders stuck to straw particles (cover straw in coating) but a lot of straw particles } \\
\text { uncoated and crunchy; some unders clumped together; still dry }\end{array}$ \\
\hline 28 & light; fluffy; still separated unders/straw \\
\hline 36 & soft, fluffy, some instances of under clumping; very little degradation \\
\hline 49 & $\begin{array}{l}\text { still separation of materials; dusty at times; some clumpls of unders; center contains most } \\
\text { of moisture }\end{array}$ \\
\hline 55 & still a mixture; light, dusty \\
\hline 63 & separate straw/unders; damp unders \\
\hline
\end{tabular}

Notes:

1) Observations made before moisture content adjustments ( $\mathrm{H}_{2} \mathrm{O}$ addition).

2) Smaller particles size may be due to brittleness of dry reactor contents. 
Phase 1 - Texture Comments Summary

Reactor $4 \mathrm{a}$ - Straw and Unders ( $55 \% \mathrm{MC})$

\begin{tabular}{|c|l|}
\hline Day & Comments \\
\hline 1 & less dry than $3 a, 3 b$ \\
\hline 17 & less unders clumping than $3 a, 3 b ;$ wetter feel; longer straw fibres than $3 a, 3 b$ \\
\hline 21 & more of a mixture than $3 z, 3 b ;$ soft \\
\hline 24 & a lot less "straw-like" mixture - actually forming a composted material \\
\hline 28 & fairly dry; noteiceable straw particles \\
\hline 36 & light, soft; some noticeable straw particles \\
\hline 49 & $\begin{array}{l}\text { quite soft; less clumping than } 3 a, b ; \text { top surface drier - middle moist; still recognizable } \\
\text { material separation }\end{array}$ \\
\hline 55 & more dense than 3a,b \\
\hline 63 & soft; light; some harder straw particles \\
\hline
\end{tabular}

Reactor $4 \mathrm{~b}$ - Straw and Unders (55\% MC)

\begin{tabular}{|c|l|}
\hline Day & lomments \\
\hline 1 & less dry than $3 \mathrm{a}, 3 \mathrm{~b}$ \\
\hline 17 & similar to $3 \mathrm{a}, 3 \mathrm{~b}$ but wetter \\
\hline 21 & more of a mixture than $3 \mathrm{z}, 3 \mathrm{~b} ;$ soft \\
\hline 24 & same as 4a but drier \\
\hline 28 & a little wetter and softer than $4 \mathrm{a} ;$ more of a mixture than $4 \mathrm{a}$ \\
\hline 36 & light, soft; some noticeable straw particles \\
\hline 49 & $\begin{array}{l}\text { quite soft; less clumping than } 3 \mathrm{a}, \mathrm{b} ; \text { top surface drier - middle moist; still recognizable } \\
\text { material separation }\end{array}$ \\
\hline 55 & more dense than 3a,b \\
\hline 63 & soft; light; some harder straw particles \\
\hline
\end{tabular}

Notes:

1) Observations made before moisture content adjustments $\left(\mathrm{H}_{2} \mathrm{O}\right.$ addition).

2) Smaller particles size may be due to brittleness of dry reactor contents. 
Phase 1 - Texture Comments Summary

Reactor 5a - Straw and Unders ( $62.5 \% \mathrm{MC})$

\begin{tabular}{|c|c|}
\hline Day & Comments \\
\hline 1 & \multirow{9}{*}{$\begin{array}{l}\text { less dry than } 4 \mathrm{a}, 4 \mathrm{~b} \\
\text { feels more like "soil"; softer than } 1,2,3,4 \text {; not just crunchy straw } \\
\text { soft, fluffy; wetter than } 4 \mathrm{a}, 4 \mathrm{~b} \text {; some larger straw particles } \\
\text { drier than } 5 \mathrm{~b} \\
\text { very dry } \\
\text { some larger straw particles but darker in color; soft, light, fluffy } \\
\text { very light; more dense mixture } \\
\text { denser than } 4 \mathrm{a}, \mathrm{b} \\
\text { same as } 4 \mathrm{a}, \mathrm{b} \text {; less hard straw particles }\end{array}$} \\
\hline 17 & \\
\hline 21 & \\
\hline 24 & \\
\hline 28 & \\
\hline 36 & \\
\hline 49 & \\
\hline 55 & \\
\hline 63 & \\
\hline
\end{tabular}

Reactor $5 b$ - Straw and Unders (62.5\% MC)

\begin{tabular}{|c|l|}
\hline Day & Comments \\
\hline 1 & less dry than $4 \mathrm{a}, 4 \mathrm{~b}$ \\
\hline 17 & crunchy like $3 \mathrm{a}, 3 \mathrm{~b} ;$ rather dry \\
\hline 21 & soft, fluffy; wetter than $4 \mathrm{a}, 4 \mathrm{~b} ;$ some larger straw particles \\
\hline 24 & softer than $4 \mathrm{a}, \mathrm{b} ;$ more of a solid (thicker) material than $4 \mathrm{a}, \mathrm{b}$ \\
\hline 28 & very dry \\
\hline 36 & some larger straw particles but darker in color; soft, light, fluffy \\
\hline 49 & very light; more dense mixture \\
\hline 55 & more dense than $3 a, b$ \\
\hline 63 & same as $4 a, b ;$ less hard straw particles \\
\hline
\end{tabular}

Notes:

1) Observations made before moisture content adjustments $\left(\mathrm{H}_{2} \mathrm{O}\right.$ addition).

2) Smaller particles size may be due to brittleness of dry reactor contents. 
Phase 1 - Texture Comments Summary

Reactor $6 \mathrm{a}$ - Straw and Unders (70\% MC)

\begin{tabular}{|c|l|}
\hline Day & Comments \\
\hline 1 & less dry than $5 \mathrm{a}, 5 \mathrm{~b}$ \\
\hline 17 & softer mix on straw/unders; straw/unders combined; wet feeling especially in core \\
\hline 21 & soft, wet with some larger particles \\
\hline 24 & straw particles haven't decreased much in size; more soil-like; wetter \\
\hline 28 & very dry \\
\hline 36 & damp; about the same as $5 a, b$ \\
\hline 49 & very dry; hard; crunchy; some clumping \\
\hline 55 & crunchy on top; fairly wet; chunky unders \\
\hline 63 & wet; globules; little distinction between straw/unders in globules \\
\hline
\end{tabular}

Reactor $6 \mathrm{~b}$ - Straw and Unders (70\% MC)

\begin{tabular}{|c|l|}
\hline Day & Comments \\
\hline 1 & less dry than $5 \mathrm{a}, 5 \mathrm{~b}$ \\
\hline 17 & softer mix on straw/unders; straw/unders combined; wet feeling especially in core \\
\hline 21 & drier than 6a; crunchy particles \\
\hline 24 & straw particles haven't decreased much in size; more soil-like; wetter \\
\hline 28 & very dry \\
\hline 36 & damp; about the same as $5 \mathrm{a}, \mathrm{b}$ \\
\hline 49 & very dry; hard; crunchy; some clumping \\
\hline 55 & crunchy on top; fairly wet; chunky unders \\
\hline 63 & wet; globules; little distinction between straw/unders in globules \\
\hline
\end{tabular}

Notes:

1) Observations made before moisture content adjustments $\left(\mathrm{H}_{2} \mathrm{O}\right.$ addition).

2) Smaller particles size may be due to brittleness of dry reactor contents. 
Phase 1 - Texture Comments Summary

Reactor 7a - Straw, Unders, \& Lagoon (62.5\% MC)

\begin{tabular}{|c|l|}
\hline Day & lomments \\
\hline 1 & similar to $5 \mathrm{a}, 5 \mathrm{~b}$ \\
\hline 17 & soft, as $5 \mathrm{a}$ \\
\hline 21 & a little drier than $7 \mathrm{~b}$ \\
\hline 24 & drier than $7 \mathrm{~b} ;$ about the same as $5 \mathrm{a}, \mathrm{b}$ \\
\hline 28 & very dry \\
\hline 36 & a better mix of materials than $5 \mathrm{a}, \mathrm{b} ;$ soft,damp \\
\hline 49 & soft; fluffy \\
\hline 55 & some clumps; a bit crunchy on top; good mix \\
\hline 63 & $\begin{array}{l}\text { very slightly more particle-like than } 5 \mathrm{a}, \mathrm{b} ; \text { less soft than } 5 \mathrm{a}, \mathrm{b} ; \text { basically the same as } 5 \mathrm{a}, \mathrm{b} \text { but } \\
\text { darker in colour }\end{array}$ \\
\hline
\end{tabular}

Reactor 7b - Straw, Unders, \& Lagoon (62.5\% MC)

\begin{tabular}{|c|l|}
\hline Day & Comments \\
\hline 1 & similar to $5 \mathrm{a}, 5 \mathrm{~b}$ \\
\hline 17 & dry, crunchy like 2 or 3 but with $\mathrm{a}$ uniform mixture \\
\hline 21 & similar to $5 \mathrm{a}, 5 \mathrm{~b}$ \\
\hline 24 & same as $5 \mathrm{a}, \mathrm{b}$ \\
\hline 28 & not quite as dry as $7 \mathrm{a}$ \\
\hline 36 & a better mix of materials than $5 \mathrm{a}, \mathrm{b} ;$ soft, damp \\
\hline 49 & soft; fluffy \\
\hline 55 & some clumps; a bit crunchy on top; good mix \\
\hline 63 & \begin{tabular}{l} 
very slightly more particle-like than $5 \mathrm{a}, \mathrm{b} ;$ less soft than $5 \mathrm{a}, \mathrm{b} ;$ basically the same as $5 \mathrm{a}, \mathrm{b}$ but \\
\hline
\end{tabular} \\
\hline
\end{tabular}

Notes:

1) Observations made before moisture content adjustments $\left(\mathrm{H}_{2} \mathrm{O}\right.$ addition).

2) Smaller particles size may be due to brittleness of dry reactor contents. 
Phase 1 - Colour Comments Summary

Reactor 1a - Straw Only (57\% MC)

\begin{tabular}{|c|l|}
\hline Day & Comments \\
\hline 1 & straw color \\
\hline 17 & light straw color \\
\hline 21 & brown \& darker beige particles \\
\hline 24 & light brown \\
\hline 28 & medium brown \\
\hline 36 & some light straw particles but mostly darker \\
\hline 49 & some darker and some lighter brown \\
\hline 55 & med brown \\
\hline 63 & med brown, about same as 4a,b \\
\hline
\end{tabular}

Reactor $1 \mathrm{~b}$ - Straw Only ( $57 \%$ MC)

\begin{tabular}{|c|l|}
\hline Day & Comments \\
\hline 1 & straw color \\
\hline 17 & light straw color \\
\hline 21 & dry looking straw; very light brown/beige \\
\hline 24 & light brown (a little darker than 1a) \\
\hline 28 & medium brown but a little darker \\
\hline 36 & some light straw particles but mostly darker \\
\hline 49 & some darker and some lighter brown \\
\hline 55 & med brown \\
\hline 63 & med brown, about same as 4a,b \\
\hline
\end{tabular}

Notes:

1) Observations made before moisture content adjustments ( $\mathrm{H}_{2} \mathrm{O}$ addition). 
Phase 1 - Colour Comments Summary

Reactor 2a - Straw Only (70\% MC)

\begin{tabular}{|c|l|}
\hline Day & Comments \\
\hline 1 & darker straw color \\
\hline 17 & darker straw colour (more moist) \\
\hline 21 & darker brown \\
\hline 24 & dark brown (a little darker than $2 \mathrm{~b}$ ) \\
\hline 28 & darker brown \\
\hline 36 & dark brown \\
\hline 49 & very dark brown \\
\hline 55 & dark brown \\
\hline 63 & very dark brown \\
\hline
\end{tabular}

Reactor $2 \mathrm{~b}$ - Straw Only ( $70 \% \mathrm{MC})$

\begin{tabular}{|c|l|}
\hline Day & Comments \\
\hline 1 & darker straw color \\
\hline 17 & darker straw colour (more moist) \\
\hline 21 & darker brown \\
\hline 24 & dark brown \\
\hline 28 & darker brown \\
\hline 36 & dark brown \\
\hline 49 & very dark brown \\
\hline 55 & dark brown \\
\hline 63 & very dark brown \\
\hline
\end{tabular}

Notes:

1) Observations made before moisture content adjustments $\left(\mathrm{H}_{2} \mathrm{O}\right.$ addition). 
Phase 1 - Colour Comments Summary

Reactor 3a - Straw and Unders (40\% MC)

\begin{tabular}{|c|l|}
\hline Day & Comments \\
\hline 1 & light beige mix \\
\hline 17 & dry, pale under/straw mix \\
\hline 21 & dry straw/under mixture \\
\hline 24 & light brown; pale \\
\hline 28 & light brown \\
\hline 36 & medium beige/light brown \\
\hline 49 & light brown/beige \\
\hline 55 & light brown \\
\hline 63 & light brown \\
\hline
\end{tabular}

Reactor $3 \mathrm{~b}$ - Straw and Unders (40\% MC)

\begin{tabular}{|c|l|}
\hline Day & Comments \\
\hline 1 & light beige mix \\
\hline 17 & dry, pale under/straw mix \\
\hline 21 & dry straw/under mixture \\
\hline 24 & light brown; pale \\
\hline 28 & light brown \\
\hline 36 & a little lighter than 3a \\
\hline 49 & light brown/beige \\
\hline 55 & light brown \\
\hline 63 & light brown \\
\hline
\end{tabular}

Notes:

1) Observations made before moisture content adjustments ( $\mathrm{H}_{2} \mathrm{O}$ addition). 
Phase 1 - Colour Comments Summary

Reactor $4 \mathrm{a}$ - Straw and Unders (55\% MC)

\begin{tabular}{|c|l|}
\hline Day & Comments \\
\hline 1 & darker beige mix \\
\hline 17 & a little darker than $3 \mathrm{a}, 3 \mathrm{~b}$ \\
\hline 21 & more uniform colour of medium brown than $3 \mathrm{a}, 3 \mathrm{~b}$ \\
\hline 24 & darker brown than $3 \mathrm{a}, \mathrm{b}$ \\
\hline 28 & a little darker than $3 \mathrm{a}, \mathrm{b}$ \\
\hline 36 & medium brown \\
\hline 49 & medium brown \\
\hline 55 & med to dark brown, some lighter straw particles \\
\hline 63 & med brown \\
\hline
\end{tabular}

Reactor $4 \mathrm{~b}$ - Straw and Unders ( $55 \% \mathrm{MC})$

\begin{tabular}{|c|l|}
\hline Day & Comments \\
\hline 1 & darker beige mix \\
\hline 17 & very dry like $3 a$ \\
\hline 21 & more uniform colour of medium brown than $3 a, 3 b$ \\
\hline 24 & darker than $3 a, b$ but lighter than $4 a$ \\
\hline 28 & a little darker than $3 a, b$ \\
\hline 36 & medium brown \\
\hline 49 & medium brown \\
\hline 55 & med to dark brown, some lighter straw particles \\
\hline 63 & med brown \\
\hline
\end{tabular}

Notes:

1) Observations made before moisture content adjustments ( $\mathrm{H}_{2} \mathrm{O}$ addition). 
Phase 1 - Colour Comments Summary

Reactor 5a - Straw and Unders (62.5\% MC)

\begin{tabular}{|c|l|}
\hline Day & Comments \\
\hline 1 & brown mix \\
\hline 17 & darker than 4 \\
\hline 21 & a little darker brown than $4 \mathrm{a}, 4 \mathrm{~b}$ \\
\hline 24 & about the same as $4 \mathrm{a}, \mathrm{b}$ \\
\hline 28 & a little darker than $4 \mathrm{a}, \mathrm{b}$ \\
\hline 36 & darker than $4 \mathrm{a}, \mathrm{b}$ \\
\hline 49 & med-dark brown \\
\hline 55 & darker brown than $4 \mathrm{a}, \mathrm{b} ;$ lesser amounts of light straw particles \\
\hline 63 & dark brown \\
\hline
\end{tabular}

Reactor $5 b$ - Straw and Unders (62.5\% MC)

\begin{tabular}{|c|l|}
\hline Day & lomments \\
\hline 1 & brown mix \\
\hline 17 & between 3 and 4 \\
\hline 21 & a little darker brown than $4 a, 4 b$ \\
\hline 24 & darker brown than $4 a, b$ \\
\hline 28 & a little darker than $4 a, b$ \\
\hline 46 & darker than $4 a, b$ \\
\hline 55 & med-dark brown \\
\hline 63 & darker brown than $4 a, b ;$ lesser amounts of light straw particles \\
\hline
\end{tabular}

Notes:

1) Observations made before moisture content adjustments $\left(\mathrm{H}_{2} \mathrm{O}\right.$ addition). 
Phase 1 - Colour Comments Summary

Reactor 6a - Straw and Unders (70\% MC)

\begin{tabular}{|c|l|}
\hline Day & Comments \\
\hline 1 & darker brown mix \\
\hline 17 & darker than $1-5$ \\
\hline 21 & darker brown than $5 a, 5 b$ \\
\hline 24 & dark brown \\
\hline 28 & medium brown/beige and a little darker than $5 a, b$ \\
\hline 36 & darker than $5 a, b$ \\
\hline 49 & pale brown \\
\hline 55 & darker brown than $5 a, b$ \\
\hline 63 & very dark brown \\
\hline
\end{tabular}

Reactor $6 \mathrm{~b}$ - Straw and Unders (70\% MC)

\begin{tabular}{|c|l|}
\hline Day & Comments \\
\hline 1 & darker brown mix \\
\hline 17 & darker than $1-5$ \\
\hline 21 & brown as $5 \mathrm{a}, 5 \mathrm{~b}$ \\
\hline 24 & dark brown \\
\hline 28 & medium brown/beige and a little darker than $5 \mathrm{a}, \mathrm{b}$ \\
\hline 36 & darker than $5 \mathrm{a}, \mathrm{b}$ \\
\hline 49 & pale brown \\
\hline 55 & darker brown than $5 \mathrm{a}, \mathrm{b}$ \\
\hline 63 & very dark brown \\
\hline
\end{tabular}

Notes:

1) Observations made before moisture content adjustments ( $\mathrm{H}_{2} \mathrm{O}$ addition). 


\section{Phase 1 - Colour Comments Summary}

Reactor 7a - Straw, Unders, \& Lagoon (62.5\% MC)

\begin{tabular}{|c|l|}
\hline Day & Comments \\
\hline 1 & darker brown mix \\
\hline 17 & darker, like $5 \mathrm{a}$ \\
\hline 21 & a little lighter than $7 \mathrm{~b}$ \\
\hline 24 & same as $5 \mathrm{a}, \mathrm{b}$ \\
\hline 28 & darker than $3-6$ \\
\hline 36 & darker than $3-6$ \\
\hline 49 & darker brown \\
\hline 55 & med brown \\
\hline 63 & darker than $5 \mathrm{a}, \mathrm{b}$ \\
\hline
\end{tabular}

Reactor $7 \mathrm{~b}$ - Straw, Unders, \& Lagoon (62.5\% MC)

\begin{tabular}{|c|l|}
\hline Day & Comments \\
\hline 1 & darker brown mix \\
\hline 17 & dry, pale combination \\
\hline 21 & same as $5 a, 5 b$ \\
\hline 24 & same as $5 a, b$ \\
\hline 28 & darker than $3-6$ \\
\hline 36 & darker than $3-6$ \\
\hline 49 & darker brown \\
\hline 55 & med brown \\
\hline 63 & darker than $5 a, b$ \\
\hline
\end{tabular}

Notes:

1) Observations made before moisture content adjustments ( $\mathrm{H}_{2} \mathrm{O}$ addition). 
APPENDIX D - PHASE 2 EXPERIMENTAL LABORATORY DATA 


\section{Phase 2 - Straw Feedstock Moisture Content Analysis}

Test Date: April 11, 2002

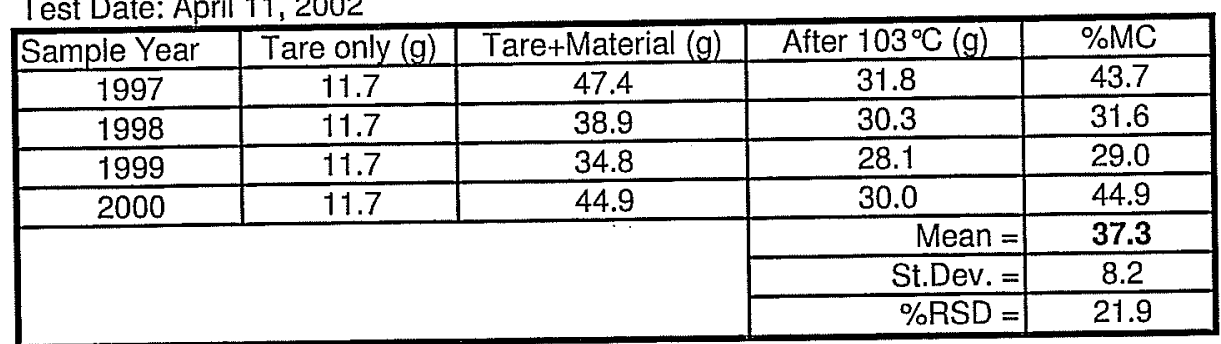

Notes:

1) Four straw bales from Dow BioProducts stackyard were sampled (years 1997 , 1998, 1999, and 2000).

2) Shredded straw samples taken April 10, 2002.

3) $\%$ RSD = St.Dev./Mean $\times 100$

Test Date: May 13, 2002

\begin{tabular}{|c|c|c|c|c|}
\hline Sample \# & Tare only $(\mathrm{g})$ & Tare+Material (g) & After $103^{\circ} \mathrm{C}(\mathrm{g})$ & $\% \mathrm{MC}$ \\
\hline Straw 2 & 11.36 & 23.52 & 20.96 & 21.1 \\
\hline Straw 2 & 11.33 & 24.77 & 22.32 & 18.2 \\
\hline Straw 2 & 11.36 & 26.29 & 23.17 & 20.9 \\
\hline & & & Mean $=$ & 20.1 \\
\hline & & & St.Dev. $=$ & 1.6 \\
\hline & & & $\% R S D=$ & 7.9 \\
\hline
\end{tabular}

Notes:

1) Unshredded straw samples taken May 2, 2002.

2) $\%$ RSD $=$ St.Dev./Mean $\times 100$

Test Date: May 14, 2002

\begin{tabular}{|c|c|c|c|c|}
\hline Sample Year & Tare only $(\mathrm{g})$ & Tare+Material $(\mathrm{g})$ & After $103^{\circ} \mathrm{C}(\mathrm{g})$ & $\% \mathrm{MC}$ \\
\hline 1997 & 11.41 & 33.58 & 23.83 & 44.0 \\
\hline
\end{tabular}

Notes:

1) Shredded straw samples taken April 10, 2002.

From Phase 1:

\begin{tabular}{|r|c|}
\hline & $\%$ MC \\
\hline Straw Mean $=$ & 57.5 \\
\hline St.Dev. $=$ & 16.6 \\
\hline$\%$ RSD $=$ & 29.0 \\
\hline
\end{tabular}




\section{Phase 2 - Straw Feestock Solids Analysis}

Test Date: April 22, 2002

\begin{tabular}{|c|c|c|c|c|c|c|c|}
\hline & & Tare & Tare + Dry Straw & After $550^{\circ} \mathrm{C}(\mathrm{g})$ & VS & $\mathrm{FS}$ & OC \\
\hline Sample Year & Crucible \# & (g) & (g) & $(g)$ & $(\%)$ & $(\%)$ & $(\%)$ \\
\hline \multirow{3}{*}{1997} & $\mathrm{~T} 2$ & 88.1443 & 91.8020 & 88.5801 & 88.09 & 11.91 & \\
\hline & T3 & 87.3280 & 91.3949 & 87.8134 & 88.06 & 11.94 & \\
\hline & $T 4$ & 87.4976 & 90.8428 & 87.9305 & 87.06 & 12.94 & \\
\hline Mean & & & & & 87.74 & 12.26 & 48.7 \\
\hline St.Dev. & & & & & 0.59 & 0.59 & 0.59 \\
\hline \multirow{3}{*}{1998} & T5 & 88.6142 & 92.0152 & 89.0504 & 87.17 & 12.83 & \\
\hline & $\mathrm{T1}$ & 87.7727 & 91.3728 & 88.2660 & 86.30 & 13.70 & \\
\hline & T10 & 88.3252 & 91.6232 & 88.7506 & 87.10 & 12.90 & \\
\hline Mean & & & & & 86.86 & 13.14 & 48.3 \\
\hline St.Dev. & & & & & 0.49 & 0.49 & 0.49 \\
\hline \multirow{3}{*}{1999} & $\mathrm{J5}$ & 85.1650 & 88.7665 & 85.6291 & 87.11 & 12.89 & \\
\hline & 56 & 89.9660 & 93.1802 & 90.3630 & 87.65 & 12.35 & \\
\hline & 43 & 83.7480 & 86.1434 & 84.0264 & 88.38 & 11.62 & \\
\hline Mean & & & & & 87.71 & 12.29 & 48.7 \\
\hline St.Dev. & & & & & 0.63 & 0.63 & 0.63 \\
\hline \multirow{3}{*}{2000} & 39 & 90.3469 & 93.7285 & disturbed & - & - & \\
\hline & $T 8$ & 88.0326 & 91.0184 & 88.3256 & 90.19 & 9.81 & \\
\hline & $\mathrm{T} 11$ & 87.8929 & 90.9765 & 88.1998 & 90.05 & 9.95 & \\
\hline Mean & & & & & 90.12 & 9.88 & 50.1 \\
\hline St.Dev. & & & & & 0.10 & 0.10 & 0.10 \\
\hline
\end{tabular}

Notes:

1) Straw samples collected on April 10,2002.

2) OC (Organic Carbon) calculated using the following equation: $O C=(1-F S) / 1.8$ (Haug 1993, Liao 1995), where

$O C$ and $F S$ are based on the dry weight fraction.

Values Used for Phase 2:

Values Used for Phase 2:
\begin{tabular}{|r|c|c|c|}
\hline & $\%$ VS & $\%$ FS & $\%$ OC $(\mathrm{db})$ \\
\hline Straw Mean $=$ & 88.1 & 11.9 & 48.9 \\
\hline St.Dev. $=$ & 1.4 & 1.4 & 0.8 \\
\hline \%RSD $=$ & 1.6 & 11.8 & 1.6 \\
\hline
\end{tabular}

Values Used for Phase 1:

\begin{tabular}{|r|c|c|}
\hline & $\%$ VS: & $\%$ OC $(\mathrm{db})$ \\
\hline Straw Mean $=$ & 81.0 & 45.0 \\
\hline St.Dev. $=$ & 1.7 & 0.9 \\
\hline \%RSD $=$ & 2.1 & 2.1 \\
\hline
\end{tabular}


Phase 2 - Straw Feedstock TKN Analysis

Test Date: April 23-25, 2002

Standards:

\begin{tabular}{|c|c|c|}
\hline $\mathrm{mg} N / \mathrm{L}$ & \# of Squares & Adjusted \\
\hline 75 & 94 & 93 \\
\hline 50 & 71 & 70 \\
\hline 25 & 37 & 36 \\
\hline 10 & 15 & 14 \\
\hline 5 & 8 & 7 \\
\hline blank & 1 & 0 \\
\hline
\end{tabular}

Conversion Equation:

\# squares $/ 1.3014=\mathrm{mg} \mathrm{N} / \mathrm{L}$

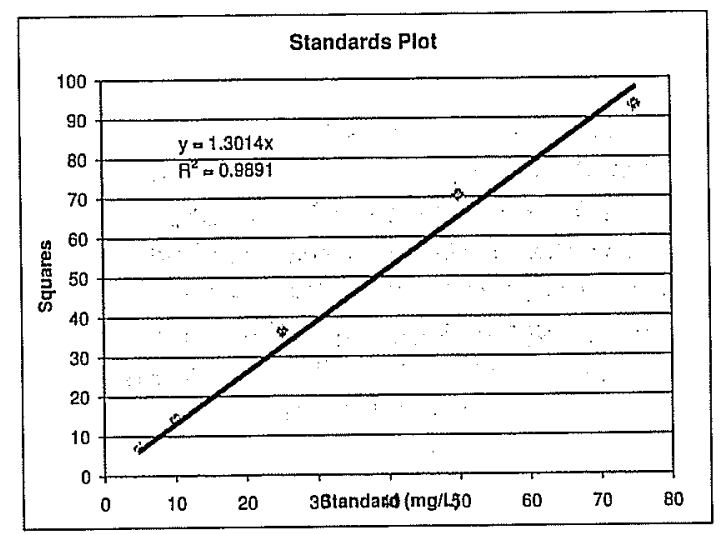

\begin{tabular}{|c|c|c|c|c|c|c|c|c|}
\hline & Sample Size (mg) & \# of Squares & $\mathrm{mg} \mathrm{N} / \mathrm{L}$ & $N$ in sample & $\% \mathrm{~N}(\mathrm{db})$ & $\mathrm{gN} / \mathrm{g}$ ash & $\% O C(\mathrm{db})$ & $\mathrm{C}: \mathrm{N}$ \\
\hline Straw Sample Year & $(\mathrm{mg})$ & & & $(\mathrm{mg})$ & (dry basis) & (dry basis) & (dry basis) & \\
\hline \multirow{3}{*}{1997} & 100 & 21 & 16.14 & 0.807 & 0.81 & 0.07 & & \\
\hline & 100 & 20 & 15.37 & 0.768 & 0.77 & 0.06 & & \\
\hline & 100 & 21 & 16.14 & 0.807 & 0.81 & 0.07 & & \\
\hline \multirow{3}{*}{1998} & 100 & 23 & 17.67 & 0.884 & 0.88 & 0.07 & & \\
\hline & 100 & 18 & 13.83 & 0.692 & 0.69 & 0.06 & & \\
\hline & 100 & 20 & 15.37 & 0.768 & 0.77 & 0.06 & & \\
\hline \multirow{3}{*}{1999} & 100 & 18 & 13.83 & 0.692 & 0.69 & 0.06 & & \\
\hline & 100 & 19 & 14.60 & 0.730 & 0.73 & 0.06 & & \\
\hline & 100 & 21 & 16.14 & 0.807 & 0.81 & 0.07 & & \\
\hline \multirow{3}{*}{2000} & 100 & 18 & 13.83 & 0.692 & 0.69 & 0.06 & & \\
\hline & 100 & 18 & 13.83 & 0.692 & 0.69 & 0.06 & & \\
\hline & 100 & 21 & 16.14 & 0.807 & 0.81 & 0.07 & & \\
\hline \multirow{3}{*}{+2} & & & & Average $=$ & 0.76 & 0.06 & 48.9 & 64.2 \\
\hline & & & & Std. Dev. $=$ & 0.06 & 0.01 & 0.8 & \\
\hline & & & & $\%$ ASD $=$ & 8.28 & 8.28 & 1.6 & \\
\hline
\end{tabular}

Notes:

1) Shredded Straw Samples taken April 10, 2002

2) Samples were dried and grinded prior to TKN analysis.

3) OC (Organic Carbon) calculated using the following equation: OC $=(1-F S) / 1.8$ (Haug 1993, Liao 1995), where OC and FS are

based on the dry weight fraction.

4) $\%$ ASD $=$ St.Dev. $/$ Mean $\times 100$

Literature Ranges (Rynk, 1992):

Literature Ranges (Rynk, 1992):
\begin{tabular}{|c|c|c|}
\hline$\% \mathrm{~N}(\mathrm{db})$ & $\% \mathrm{OC}(\mathrm{db})$ & $\mathrm{C}: \mathrm{N}$ \\
\hline $0.3-1.1$ & $14-165$ & $48-150$ \\
\hline
\end{tabular}

From Phase 1:

\begin{tabular}{|r|c|c|c|}
\hline & $\% \mathrm{~N}(\mathrm{db})$ & $\% O \mathrm{C}(\mathrm{db})$ & $\mathrm{C}: \mathrm{N}$ \\
\hline Straw Mean $=$ & 1.43 & 45.0 & 31.5 \\
\hline Std Dev. $=$ & 0.23 & 0.9 & \\
\hline \%RSD $=$ & 16.2 & 2.1 & \\
\hline
\end{tabular}




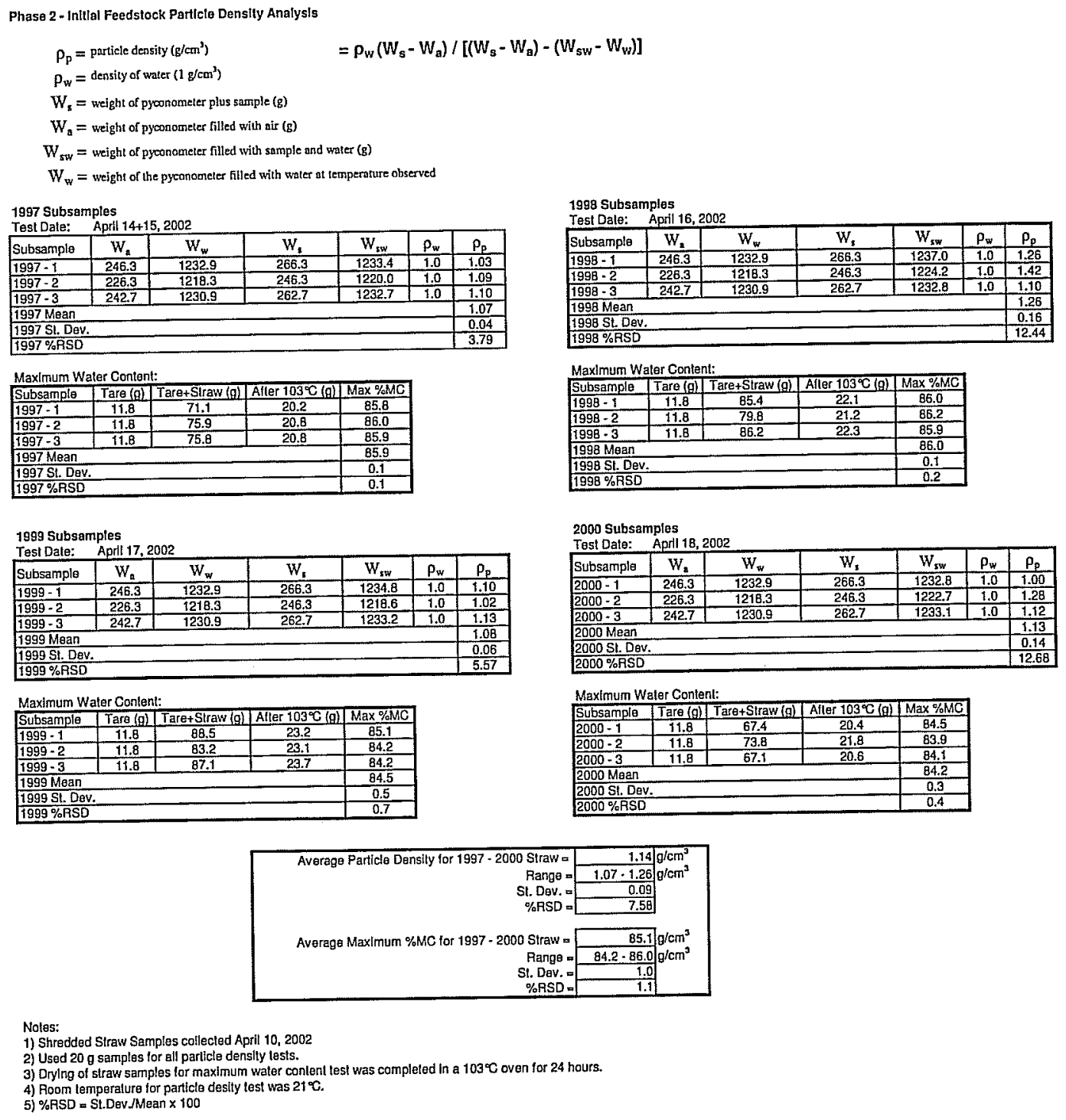


Phase 2 - Straw Feedstock Bulk Density Analysis

\begin{tabular}{|c|c|c|c|c|c|c|c|}
\hline Biocell ID & Tare $(\mathrm{kg})$ & Volume (L) & Tare+Straw $(\mathrm{kg})$ & Straw $(\mathrm{kg})$ & $\begin{array}{c}\text { Wel Bulk Density } \\
\left(\mathrm{kg} / \mathrm{m}^{3}\right)\end{array}$ & $\% \mathrm{MC}$ & $\begin{array}{c}\text { Dry Bulk Density } \\
\left(\mathrm{kg} / \mathrm{m}^{3}\right)\end{array}$ \\
\hline 1 & 1.65 & 4.33 & 1.96 & 0.31 & 71.59 & 43.70 & 40.31 \\
\hline 2 & i.64 & 4.33 & 1.96 & 0.32 & 73.90 & 43.70 & 41.61 \\
\hline 3 & 1.64 & 4.33 & 1.94 & 0.30 & 69.28 & 43.70 & 39.01 \\
\hline \multicolumn{4}{|l|}{1997 Mean } & 0.31 & 71.59 & & 40.31 \\
\hline \multicolumn{5}{|l|}{1997 St. Dev. } & 2.31 & & 1.30 \\
\hline \multicolumn{5}{|l|}{$1997 \%$ RSD } & 3.23 & & 3.23 \\
\hline
\end{tabular}

1998 Shredded Straw
\begin{tabular}{|c|c|c|c|c|c|c|c|}
\hline Blocell ID & Tare $(\mathrm{kg})$ & Volume $(\mathrm{L})$ & Tare+Straw $(\mathrm{kg})$ & Straw $(\mathrm{kg})$ & $\begin{array}{c}\text { Wet Bulk Densily } \\
\left(\mathrm{kg} / \mathrm{m}^{3}\right)\end{array}$ & $\begin{array}{c}\text { Dry Bulk Densily } \\
\left(\mathrm{kg} / \mathrm{m}^{3}\right)\end{array}$ \\
\hline 1 & 1.65 & 4.33 & 1.92 & 0.27 & 62.36 & 31.62 & 42.64 \\
\hline 2 & 1.64 & 4.33 & 1.89 & 0.25 & 57.74 & 31.62 & 39.48 \\
\hline 3 & 1.64 & 4.33 & 1.89 & 0.25 & 57.74 & 31.62 & 39.48 \\
\hline 1998 Mean & & & 0.26 & 59.28 & & 40.53 \\
\hline 1998 St. Dev.
\end{tabular}

\begin{tabular}{l}
1999 Shredded Straw \\
\begin{tabular}{|c|c|c|c|c|c|c|c|}
\hline Biocell ID & Tare $(\mathrm{kg})$ & Volume $(\mathrm{L})$ & Tare+Straw $(\mathrm{kg})$ & Straw $(\mathrm{kg})$ & $\begin{array}{c}\text { Wet Bulk Density } \\
\left(\mathrm{kg} / \mathrm{m}^{3}\right)\end{array}$ & $\begin{array}{c}\text { Dry Bulk Density } \\
\left(\mathrm{kg} / \mathrm{m}^{3}\right)\end{array}$ \\
\hline 1 & 1.65 & 4.33 & 1.91 & 0.26 & 60.05 & 29.00 & 42.63 \\
\hline 2 & 1.64 & 4.33 & 1.89 & 0.25 & 57.74 & 29.00 & 40.99 \\
\hline 3 & 1.64 & 4.33 & 1.90 & 0.26 & 60.05 & 29.00 & 42.63 \\
\hline 1999 Mean & & & 0.26 & 59.28 & & 42.08 \\
\hline 1999 St. Dev.
\end{tabular} \\
\hline 1999\%RSD
\end{tabular}

\begin{tabular}{|c|c|c|c|c|c|c|c|}
\hline Biocell ID & Tare $(\mathrm{kg})$ & Volume (L) & Tare+Straw $(\mathrm{kg})$ & Straw $(\mathrm{kg})$ & $\begin{array}{c}\text { Wet Bulk Denslly } \\
\left(\mathrm{kg} / \mathrm{m}^{3}\right)\end{array}$ & $\% \mathrm{MC}$ & $\begin{array}{c}\text { Dry Bulk Density } \\
\left(\mathrm{kg} / \mathrm{m}^{3}\right)\end{array}$ \\
\hline 1 & 1.65 & 4.33 & 1.97 & 0.32 & 73.90 & 44.88 & 40.74 \\
\hline 2 & 1.64 & 4.33 & 1.95 & 0.31 & 71.59 & 44.88 & 39.46 \\
\hline 3 & 1.64 & 4.33 & 1.98 & 0.34 & 78.52 & 44.88 & 43.28 \\
\hline 2000 Mean & & & & 0.32 & 74.67 & & 41.16 \\
\hline \multirow{2}{*}{\multicolumn{5}{|c|}{$\begin{array}{l}2000 \text { St. Dev. } \\
2000 \% \text { ASD }\end{array}$}} & 3.53 & & 1.94 \\
\hline & & & & & 4.72 & & 4.72 \\
\hline
\end{tabular}

Notes:

1) Small biocell used for analyses.

2) \%RSD = St.Dev./Mean $\times 100$

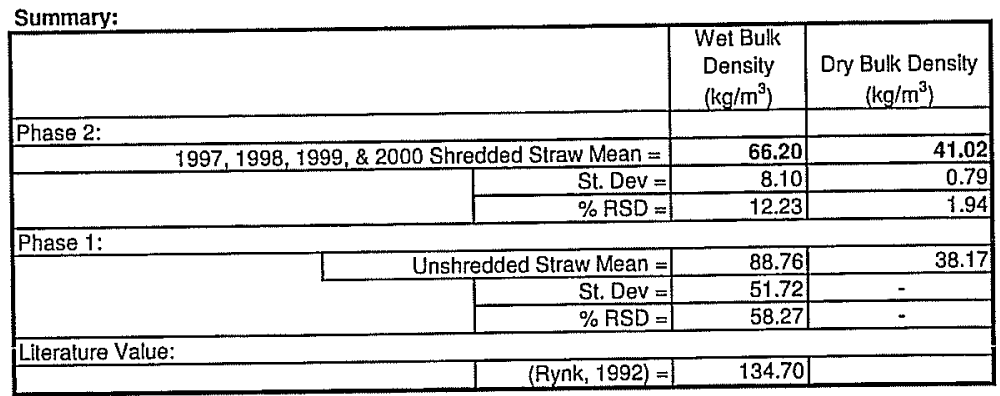


Phase 2 - Straw Feedstock Bulk Density Analysis

1997 Unshredded Straw

Test Date: May 13, 2002

Test Date: May 13,2002
\begin{tabular}{|c|c|c|c|c|c|c|}
\hline & $\begin{array}{c}\text { Volume of } \\
\text { Biocell }\left(\mathrm{m}^{3}\right)\end{array}$ & $\begin{array}{c}\text { Volume of } \\
\text { Blocell (L) }\end{array}$ & $\begin{array}{c}\text { Wet Bullk } \\
\text { Density } \\
\left(\mathrm{kg} / \mathrm{m}^{3}\right)\end{array}$ & $\%$ Straw in Biocell $(\mathrm{kg})$ & $\begin{array}{c}\text { Dry Bulk } \\
\text { Density } \\
\left(\mathrm{kg} / \mathrm{m}^{3}\right)\end{array}$ \\
\hline L1 & 0.3109 & 310.9 & 7.00 & 22.52 & 20.06 & 18.00 \\
\hline
\end{tabular}

Noles:

1) Large biocell used for analyses.

2) Unshredded straw samples collected on May 2, 2002 were used for the analyses.

1997 Shredded Straw

Tes! Date: May 14, 2002

Tes! Date: May 14,2002
\begin{tabular}{|c|c|c|c|c|c|c|}
\hline & $\begin{array}{c}\text { Volume of } \\
\text { Biocell }\left(\mathrm{m}^{3}\right)\end{array}$ & $\begin{array}{c}\text { Volume of } \\
\text { Biocell }(\mathrm{L})\end{array}$ & Straw in Biocell $(\mathrm{kg})$ & $\begin{array}{c}\text { Wet Bulk } \\
\text { Densily } \\
\left(\mathrm{kg} / \mathrm{m}^{3}\right)\end{array}$ & $\%$ Bry Bulk \\
\hline $\mathrm{LI}$ & 0.3109 & 310.9 & 34.15 & 109.84 & 43.98 & $\begin{array}{c}\text { Density } \\
\left(\mathrm{kg} / \mathrm{m}^{3}\right)\end{array}$ \\
\hline
\end{tabular}

Notes:

1) Large biocell used for analyses.

2) Unshredded straw samples collected on May 2, 2002 were used for the analyses. 
Phase 2 - Compost Recipe Bulk Density Analysis

Recipe 1 - Unshredded Straw

Test Date: May 23, 2002

Test Date: May 23, 2002
\begin{tabular}{|c|c|c|c|c|c|c|}
\hline & $\begin{array}{c}\text { Volume of } \\
\text { Blocell }\left(\mathrm{m}^{3}\right)\end{array}$ & $\begin{array}{c}\text { Volume of } \\
\text { Biacell }(\mathrm{L})\end{array}$ & Straw in Biocell $(\mathrm{kg})$ & $\begin{array}{c}\text { Wet Bulk } \\
\text { Density } \\
\left(\mathrm{kg} / \mathrm{m}^{3}\right)\end{array}$ & $\begin{array}{c}\text { Dry Bulk } \\
\text { Density } \\
\left(\mathrm{kg} / \mathrm{m}^{3}\right)\end{array}$ \\
\hline Large Biocell & 0.3109 & 310.9 & 7.82 & 25.15 & 20.06 & 20.11 \\
\hline
\end{tabular}

Notes:

1) Large blocell used for analyses.

2) Unshredded 1997 straw samples were used for the analyses.

Reclpe 2 - 2/3 Unshredded Straw, 1/3 shredded straw (by volume)

Tested on: May 31, 2002

\begin{tabular}{|c|r|r|r|r|r|r|}
\hline & & & $\begin{array}{c}\text { Wet Bulk } \\
\text { Density } \\
\left(\mathrm{kg} / \mathrm{m}^{3}\right)\end{array}$ & $\%$ Dry Bulk \\
Large Biocell & Volume $\left(\mathrm{m}^{3}\right)$ & Volume $(\mathrm{L})$ & Straw Added $(\mathrm{kg})$ & $\begin{array}{r}\text { Density } \\
\left(\mathrm{kg} / \mathrm{m}^{3}\right)\end{array}$ \\
\hline L1 & 0.3109 & 310.9 & 15.38 & $\mathbf{4 9 . 4 7}$ & 36.5 & 31.41 \\
\hline
\end{tabular}

Notes:

1) Large blocell used for analyses.

2) Unshredded and shredded 1997 straw samples were used for the analyses.

Reclpe 3 - 1/3 Unshredded Straw, 2/3 shredded straw (by volume)

Tested on: May 31, 2002

\begin{tabular}{|c|r|r|r|r|r|r|}
\hline & & & $\begin{array}{c}\text { We! Bulk } \\
\text { Density } \\
\left(\mathrm{kg} / \mathrm{m}^{3}\right)\end{array}$ & $\% \mathrm{MC}$ & $\begin{array}{c}\text { Dry Bulk } \\
\text { Density } \\
\left(\mathrm{kg} / \mathrm{m}^{3}\right)\end{array}$ \\
\hline Large Biocell & Volume $\left(\mathrm{m}^{3}\right)$ & Volume $(\mathrm{L})$ & Straw Added $(\mathrm{kg})$ & 41.5 & 49.13 \\
\hline L1 & 0.2798 & 279.8 & 23.5 & 83.99 & 41.5 &
\end{tabular}

Notes:

1) Large biocell used for analyses.

2) Unshredded and shredded 1997 straw samples were used for the analyses.

Reclpe 4 - Shredded Straw

Test Date: May 23, 2002

Test Date: May 23,2002
\begin{tabular}{|c|c|c|c|c|c|c|}
\hline Large Biocell & $\begin{array}{c}\text { Volume of } \\
\text { Biocell }\left(\mathrm{m}^{3}\right)\end{array}$ & $\begin{array}{c}\text { Volume of } \\
\text { Biocell }(\mathrm{L})\end{array}$ & Straw in Biocell $(\mathrm{kg})$ & $\begin{array}{c}\text { Wet Bulk } \\
\text { Density } \\
\left(\mathrm{kg} / \mathrm{m}^{3}\right)\end{array}$ & $\%$ Dry Bulk \\
\hline $\mathrm{LI}$ & 0.3109 & 310.9 & 32.20 & 103.57 & 43.98 & $\begin{array}{c}\text { Densily } \\
\left(\mathrm{kg} / \mathrm{m}^{3}\right)\end{array}$ \\
\hline
\end{tabular}

Notes:

1) Large blocell used for analyses.

2) Shredded 1997 straw samples were used for the analyses. 
Phase 2 - Weekly Solids Analysis

June $1,2002 \quad$ Day 1

Phase 2 Feedstock:

\begin{tabular}{|c|c|c|c|}
\hline All Recipes & $\%$ VS & $\%$ FS & $\% O C$ (db) \\
\hline Mean & 88.11 & 11.89 & 48.95 \\
\hline St. Dev. & 1.40 & 1.40 & 0.78 \\
\hline Notes:
\end{tabular}

Notes:

1) Feedstock volatile solids based upon samples analyzed from $1997,1998,1999$, and 2000 straw stock.

2) OC (Organic Carbon) calculated using the following equation: $\mathrm{OC}=(1-\mathrm{FS}) / 1.8$ (Haug 1993, Liao 1995), where OC and FS are based on the dry weight ff

\section{Phase 2 - Weekly Solids Analysis}

\begin{tabular}{|c|c|c|c|c|c|c|c|c|c|c|}
\hline Recipe & Crucible \# & Tare only (g) & Tare+Material (g) & After $103^{\circ} \mathrm{C}(\mathrm{g})$ & After $550^{\circ} \mathrm{C}(\mathrm{g})$ & $\% M G$ & \%TS & \%VS & $\%$ FS & $\% \mathrm{OC}$ \\
\hline $1 \mathrm{~A}$ & $\mathrm{T2}$ & 88.1290 & 97.0500 & 91.0599 & 88.4534 & 67.15 & 32.85 & 88.93 & 11.07 & \\
\hline $1 \mathrm{~A}$ & T8 & 88.0190 & 95.2300 & 90.4795 & 88.3092 & 65.88 & 34.12 & 88.21 & 11.79 & \\
\hline \multirow{2}{*}{\multicolumn{2}{|c|}{ Recipe 1A Mean }} & & & & & 66.51 & & 88.57 & 11.43 & 49.2 \\
\hline & & & & & & 0.90 & & 0.51 & 0.51 & 0.51 \\
\hline $1 \mathrm{~B}$ & $T 1$ & 87.7609 & 96.2160 & 90.7166 & 88.0832 & 65.04 & 34.96 & 89.10 & 10.90 & \\
\hline $1 \mathrm{~B}$ & T6 & 88.2058 & 94.4640 & 90.6736 & 88.4932 & 60.57 & 39.43 & 88.35 & 11.65 & \\
\hline \multirow{2}{*}{\multicolumn{2}{|c|}{$\begin{array}{l}\text { Recipe 1B Mean } \\
\text { Recipe 1B St. Dev. }\end{array}$}} & & & & & 62.80 & & 88.72 & 11.28 & 49.3 \\
\hline & & & & & & 3.16 & & 0.52 & 0.52 & 0.52 \\
\hline $2 A$ & T3 & 87.3203 & 94.1163 & 89.6433 & 87.5859 & 65.82 & 34.18 & 88.57 & 11.43 & \\
\hline $2 \mathrm{~A}$ & 38 & 83.9541 & 91.5830 & 86.2007 & 84.1932 & 70.55 & 29.45 & 89.36 & 10.64 & \\
\hline \multicolumn{2}{|c|}{ Recipe 2A Mean } & & & & & 68.18 & & 88.96 & 11.04 & 49.4 \\
\hline \multicolumn{2}{|c|}{ Recipe 2A St. Dev. } & & & & & 3.35 & & 0.56 & 0.56 & 0.56 \\
\hline $2 B$ & faded $35-R$ & 89.8319 & 97.3028 & 92.3485 & 90.1635 & 66.31 & 33.69 & 86.82 & 13.18 & \\
\hline $2 \mathrm{~B}$ & 51 & 80.9666 & 88.6900 & 83.5737 & 81.3032 & 66.24 & 33.76 & 87.09 & 12.91 & \\
\hline \multicolumn{2}{|c|}{ Recipe 2B Mean } & & & & & 66.28 & & 86.96 & 13.04 & 48.3 \\
\hline \multicolumn{2}{|c|}{ Recipe 2B St. Dev. } & & & & & 0.05 & & 0.19 & 0.19 & 0.19 \\
\hline $3 \mathrm{~A}$ & W-01 & 85.1340 & 93.1520 & 87.9042 & 85.4811 & 65.45 & 34.55 & 87.47 & 12.53 & \\
\hline $3 A$ & $35-R$ & 87.3307 & 96.1270 & 90.2799 & 87.7007 & 66.47 & 33.53 & 87.45 & 12.55 & \\
\hline \multirow{2}{*}{\multicolumn{2}{|c|}{\begin{tabular}{|l|} 
Recipe 3A Mean \\
Recipe 3A St. Dev.
\end{tabular}}} & & & & & 65.96 & & 87.46 & 12.54 & 48.6 \\
\hline & & & & & & 0.72 & & 0.01 & 0.01 & 0.01 \\
\hline $3 \mathrm{~B}$ & $36-P$ & 70.9765 & 82.2400 & 74.1452 & 71.4216 & 71.87 & 28.13 & 85.95 & 14.05 & \\
\hline $3 \mathrm{~B}$ & $37-\mathrm{P}$ & 72.3980 & 78.1520 & 74.1118 & 72.6361 & 70.22 & 29.78 & 86.11 & 13.89 & \\
\hline \multicolumn{2}{|c|}{ Recipe 3B Mean } & & & & & 71.04 & & 86.03 & 13.97 & 47.8 \\
\hline \multicolumn{2}{|c|}{ Recipe 3B St. Dev. } & & & & & 1.17 & & 0.11 & 0.11 & 0.11 \\
\hline $4 \mathrm{~A}$ & 39 & 90.3446 & 101.6600 & 93.6064 & 90.7902 & 71.17 & 28.83 & 86.34 & 13.66 & \\
\hline $4 \mathrm{~A}$ & 32 & & & & & & & & & \\
\hline \multirow{2}{*}{\multicolumn{2}{|c|}{ Recipe 4A Mean }} & & & & & 71.17 & & 86.34 & 93.66 & 48.0 \\
\hline & & & & & & 0.00 & & 0.00 & 0.00 & 0.00 \\
\hline $4 \mathrm{~B}$ & 43 & 83.7509 & 97.9880 & 87.8747 & 84.3308 & 71.03 & 28.97 & 85.94 & 14.06 & \\
\hline $4 \mathrm{~B}$ & $J-5$ & 85.1700 & 94.3860 & 88.4845 & 85.6371 & 64.04 & 35.96 & $85.91 \mid$ & 14.09 & \\
\hline \multirow{2}{*}{\multicolumn{2}{|c|}{$\begin{array}{l}\text { Recipe 4B Mean } \\
\text { Recipe 4B St. Dev. }\end{array}$}} & & & & & 67.54 & & 85.92 & 14.08 & 47.7 \\
\hline & & & & & & 4.95 & & 0.02 & 0.02 & 0.02 \\
\hline
\end{tabular}

Notes:

1) OC (Organic Carbon) calculated using the following equation: $\mathrm{OC}=(1-\mathrm{FS}) / 1.8$ (Haug 1993, Liao 1995), where OC and FS are based on the dry weight fra 
Phase 2 - Weekly Solids Analysis

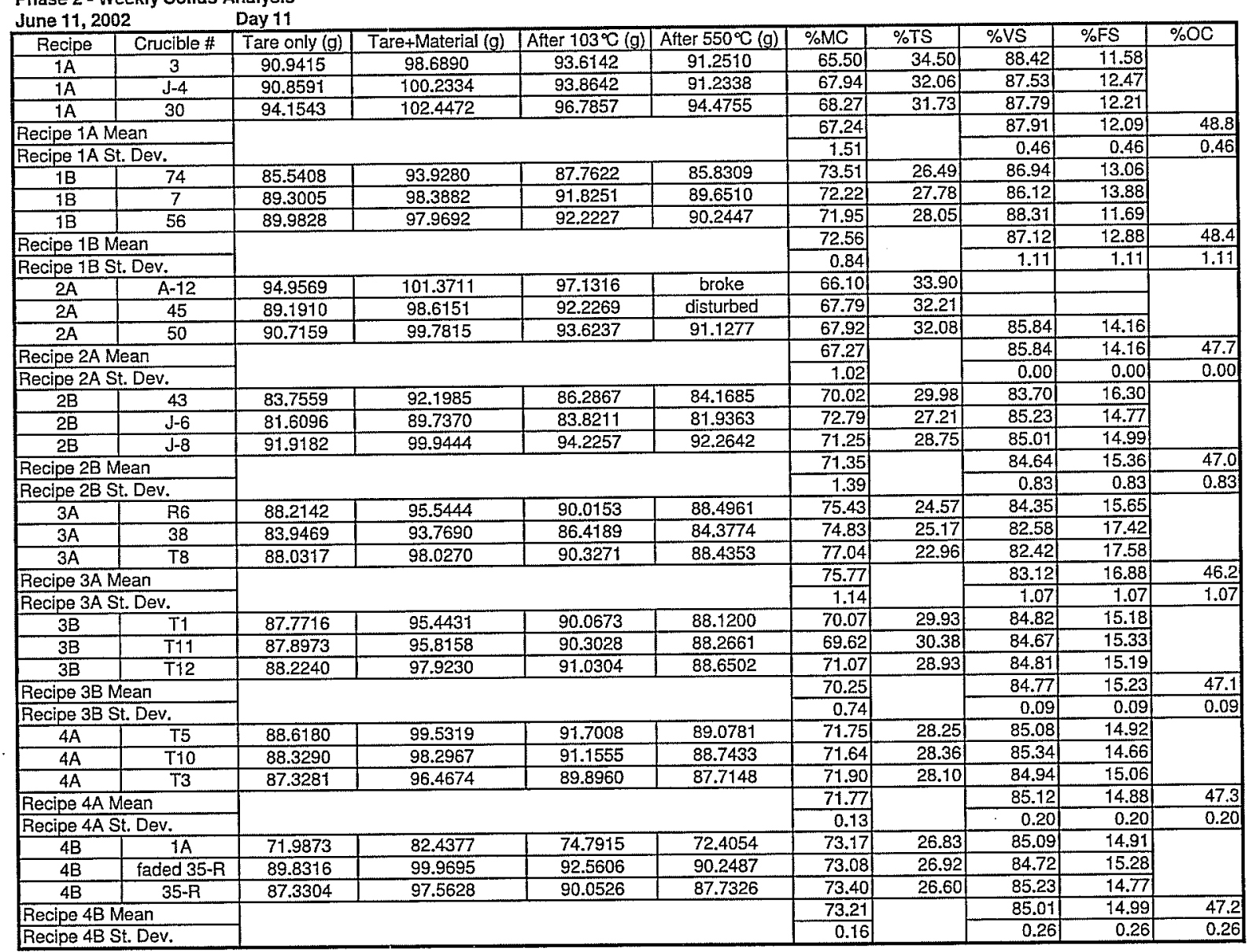

Notes:

1) OC (Organic Carbon) calculated using the following equation: $O C=(1-F S) / 1.8$ (Haug 1993, Liao 1995), where OC and FS are based on the dry weight fri 
Phase 2 - Weekly Solids Analysis

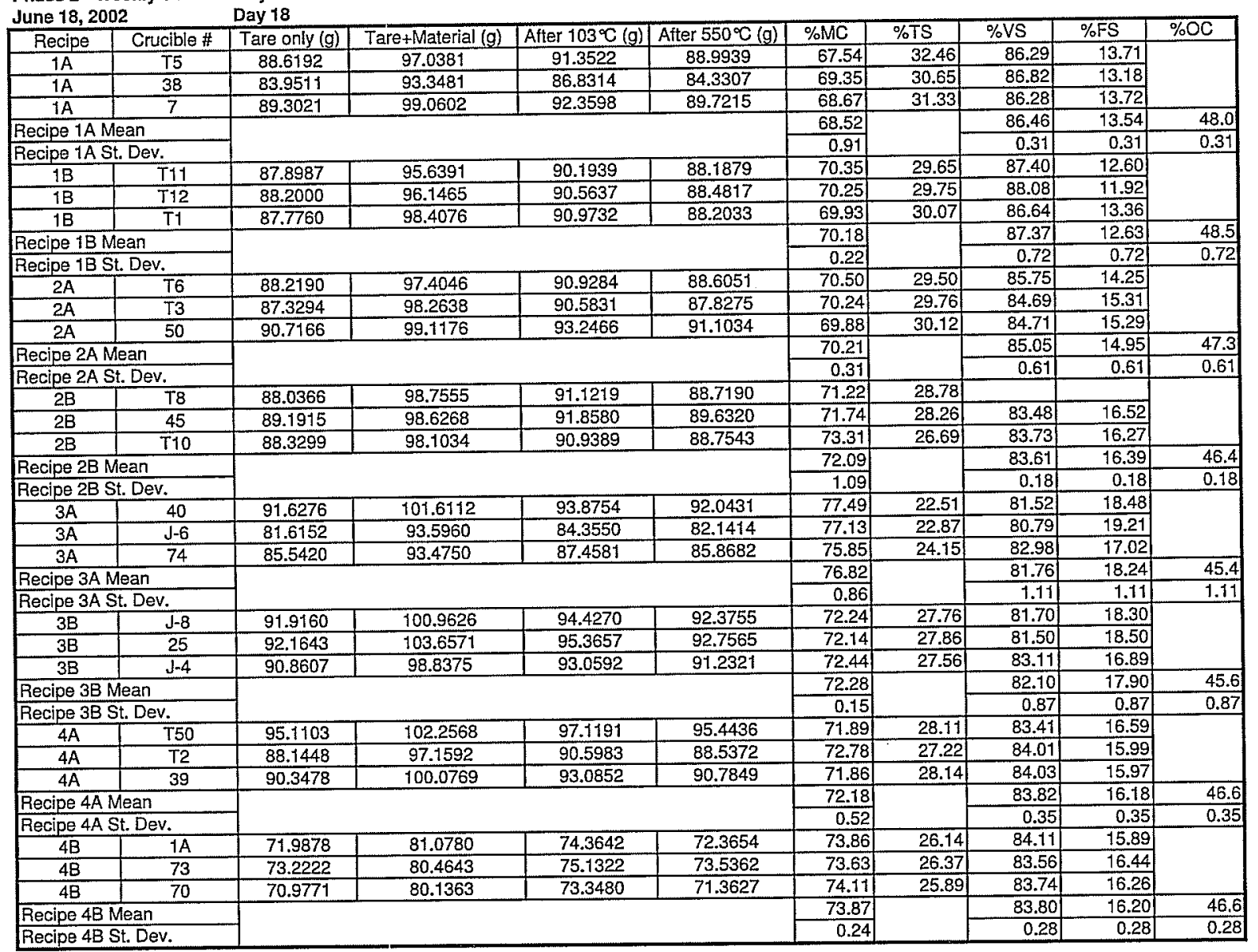

Notes:

1) OC (Organic Carbon) calculated using the following equation: OC = (1-FS)/1.8 (Haug 1993, Liao 1995), where OC and FS are based on the dry weight fri 
Phase 2 - Weekly Solids Analysis

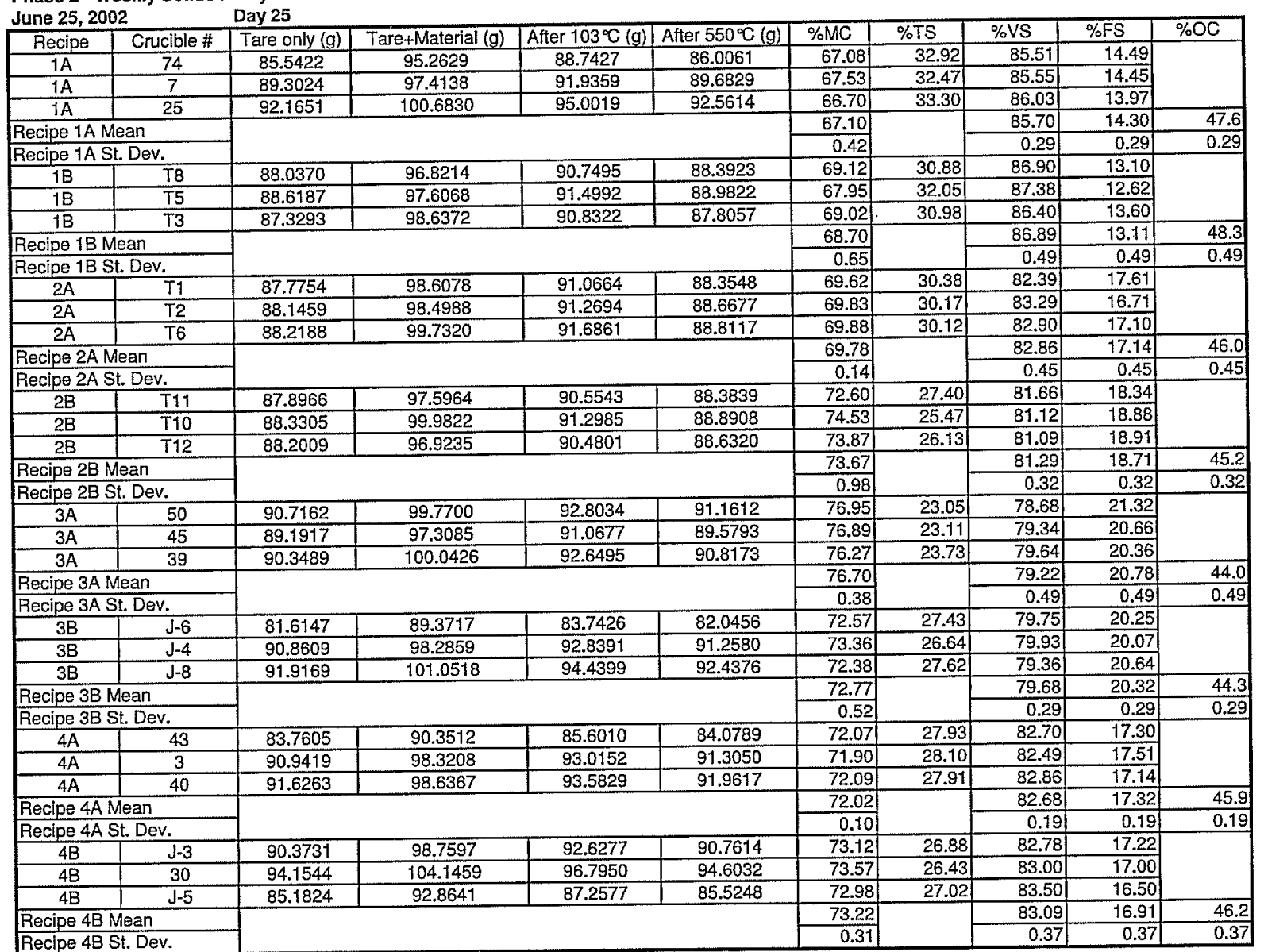

Notes:

1) OC (Organic Carbon) calculated using the following equation: OC = (1-FS)/1.8 (Haug 1993, Liao 1995), where OC and FS are based on the dry weight fri 
Phase 2 - Weekly Solids Analysis

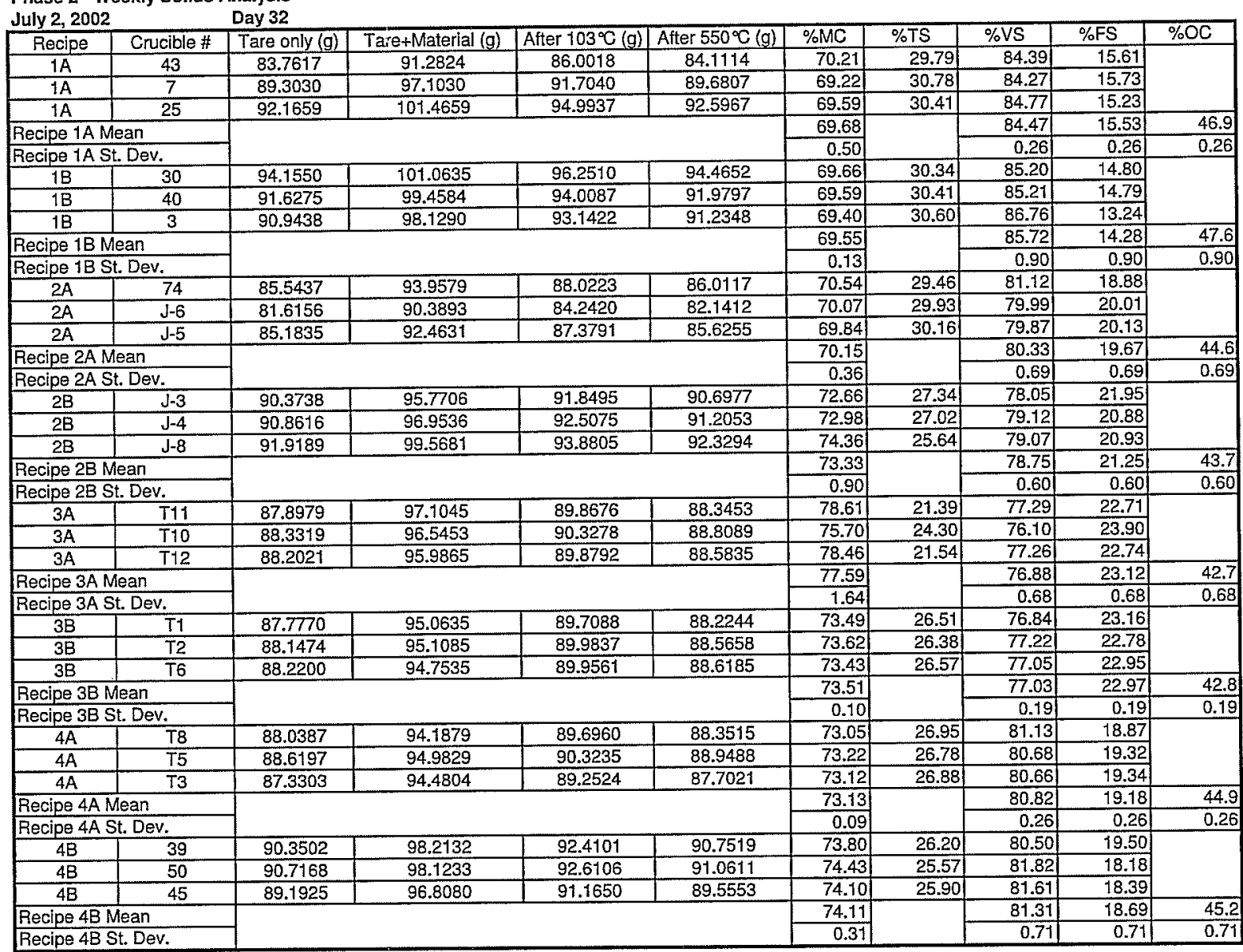

Recipe 4B St. Dev.

1) OC (Organic Carbon) calculated using the following equation: $O C=(1-F S) / 1.8$ (Haug 1993, Liao 1995), where OC and FS are based on the dry weight fri 
Phase 2 - Weekly Solids Analysis

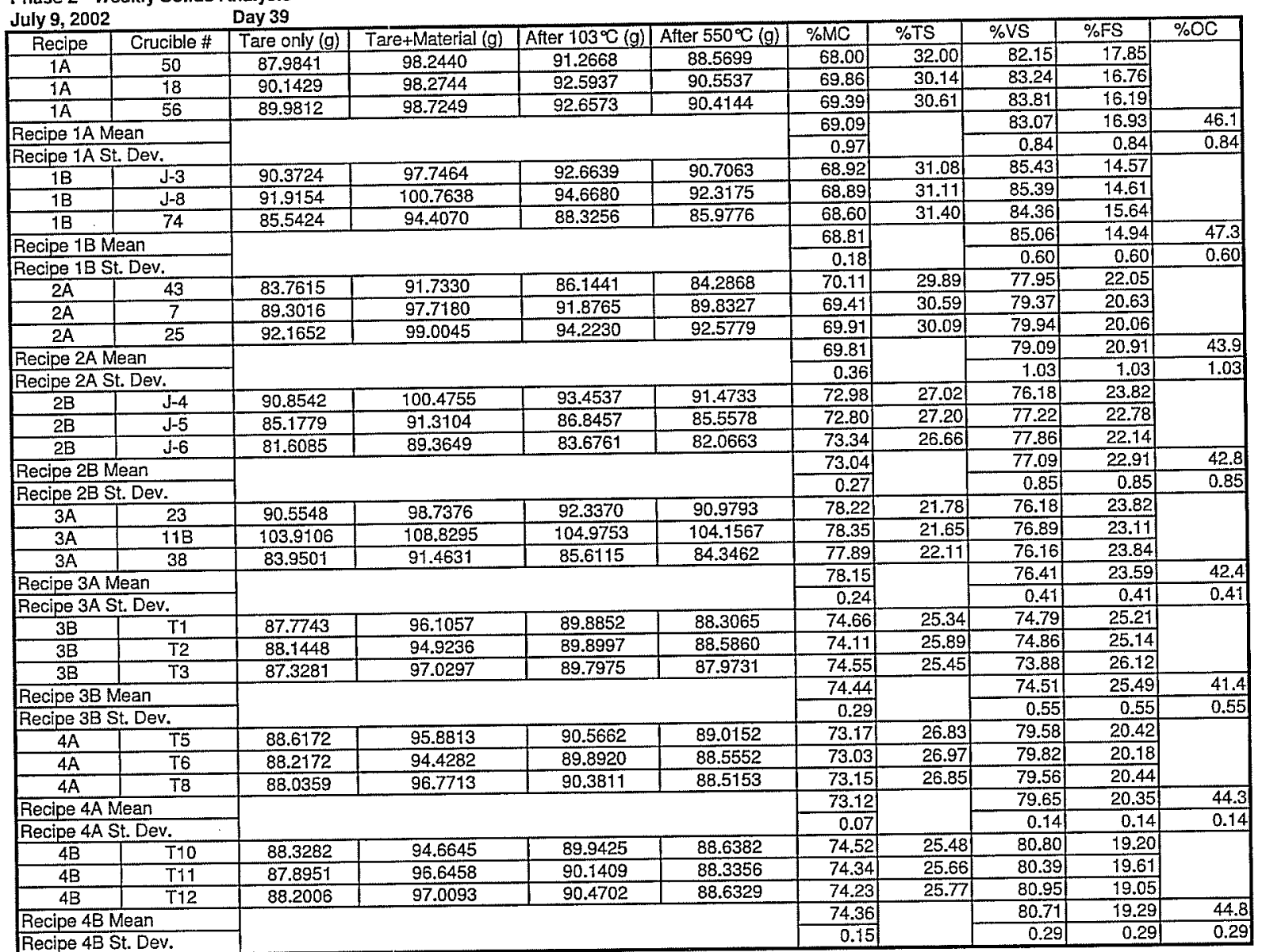

Notes:
1) OC (Organic Carbon) calculated using the following equation: $\mathrm{OC}=(1-\mathrm{FS}) / 1.8$ (Haug 1993, Liao 1995), where $\mathrm{OC}$ and FS are based on the dry weight fri 


\begin{tabular}{|c|c|c|c|c|c|c|c|c|c|c|}
\hline July 16,20 & & Day 46 & & & After $550^{\circ} \mathrm{C}(\mathrm{g})$ & $\% \mathrm{MC}$ & \%TS & $\%$ VS & $\%$ FS & $\% \mathrm{OC}$ \\
\hline $\begin{array}{c}\text { Recipe } \\
1 \mathrm{~A} \\
\end{array}$ & Crucible \# & \begin{tabular}{|l|} 
Tare only $(\mathrm{g})$ \\
\end{tabular} & $\frac{\text { Tare+Material (g) }}{97.9484}$ & After $103^{\circ} \mathrm{C}(\mathrm{g})$ & $\frac{\text { Amer } 050 \text { (9) }}{90.4427}$ & 67.22 & 32.78 & 82.40 & 17.60 & \\
\hline$\frac{1 \mathrm{~A}}{1 \mathrm{~A}}$ & 56 & 89.9832 & $\begin{array}{l}97.9484 \\
96.7774 \\
\end{array}$ & 92.59504 & 90.8988 & 67.47 & 32.53 & 83.09 & 16.91 & \\
\hline $\begin{array}{l}1 \mathrm{~A} \\
1 \mathrm{~A} \\
\end{array}$ & 23 & 90.5566 & $\frac{96.7774}{97.8179}$ & 92.5875 & 90.5742 & 68.16 & 31.84 & 82.39 & 17.61 & \\
\hline$\frac{1 \mathrm{~A}}{\text { Recipe } 1 \mathrm{AI}}$ & 18 & 90.1440 & 97.8179 & & & 67.62 & & 82.63 & 17.37 & 45.9 \\
\hline \multicolumn{2}{|c|}{ Recipe 1A Mean } & & & & & 0.48 & & 0.40 & 0.40 & 0.40 \\
\hline \multicolumn{2}{|c|}{ Recipe 1A St. Dev. } & 85.1834 & 93.7112 & 87.7383 & 85.5902 & 70.04 & 29.96 & 84.08 & 15.92 & \\
\hline$\frac{\frac{1 B}{1 B}}{1 B}$ & $\begin{array}{l}\mathrm{J}-5 \\
\mathrm{~J}-3\end{array}$ & 90.3745 & 99.0692 & 93.0601 & 90.8096 & 69.11 & 30.89 & 83.80 & 16.20 & \\
\hline$\frac{1 \mathrm{~B}}{\text { Recipe 1B }}$ & $\frac{7-8}{3-3}$ & 91.9176 & 98.7182 & 94.0605 & 92.2380 & 68.49 & 31.51 & 85.05 & 14.95 & \\
\hline & & & & & 69.21 & & 84.31 & 15.69 & 46.8 \\
\hline \multicolumn{2}{|c|}{ Recipe 1B St. Dev. } & & & & & 0.78 & & 0.66 & $\frac{0.66}{21.30}$ & \\
\hline$\frac{2 A}{2 A}$ & 74 & 85.5452 & 92.7752 & 87.7769 & 86.0206 & 69.13 & 30.87 & 78.70 & $\frac{21.30}{21.27}$ & \\
\hline$\frac{2 A}{2 A}$ & $\mathrm{~J}-4$ & 90.8613 & 97.1989 & 92.8166 & 91.2772 & 69.15 & 30.85 & 78.73 & $\frac{21.27}{20.47}$ & \\
\hline $2 A$ & J-6 & 81.6156 & 88.6713 & 83.8169 & 82.1091 & 68.80 & 31.20 & 77.58 & $\frac{22.42}{21.66}$ & \\
\hline \multirow{2}{*}{\multicolumn{2}{|c|}{$\begin{array}{l}\text { Recipe 2A Mean } \\
\text { Recipe 2A St. Dev. }\end{array}$}} & & & & & 69.03 & & $\begin{aligned} 78.34 \\
0.65 \\
\end{aligned}$ & $\frac{21.66}{0.65}$ & $\begin{array}{r}43.5 \\
0.65 \\
\end{array}$ \\
\hline & Recipe 2A St. Dev. & & & & 92.6852 & $\begin{array}{r}0.20 \\
71.15\end{array}$ & 28.85 & 73.98 & 26.02 & \\
\hline \begin{tabular}{|c|}
$2 \mathrm{~B}$ \\
$2 \mathrm{~B}$ \\
\end{tabular} & 25 & 92.1658 & 99.0834 & 94.1618 & $\frac{92.6852}{\text { broke }}$ & 73.10 & 26.90 & & & \\
\hline$\frac{2 \mathrm{~B}}{2 \mathrm{~B}}$ & 43 & 83.7614 & 93.6254 & 86.4149 & $\frac{\text { broke }}{88.4540}$ & 73.07 & 26.93 & 75.12 & 24.88 & \\
\hline$\frac{2 \mathrm{~B}}{\text { Recipe 2B }}$ & 50 & 87.9846 & 94.9928 & 89.8716 & & 72.44 & & 74.55 & 25.45 & 41.4 \\
\hline \multicolumn{2}{|c|}{ Recipe 2B Mean } & & & & & 1.12 & & 0.81 & 0.81 & 0.81 \\
\hline$\frac{\text { Recipe } 2 \mathrm{D}}{3 \mathrm{~A}}$ & Dev. & & 96.0381 & 90.3199 & 89.1250 & 77.27 & 22.73 & 71.05 & 28.95 & \\
\hline $3 A$ & $\frac{11}{T 9}$ & $\frac{88.6382}{89.7521}$ & 96.0197 & 91.1144 & 90.0908 & 78.26 & 21.74 & 75.14 & 24.86 & \\
\hline $3 A$ & 38 & 89.7521 & 90.0516 & 85.3201 & 84.3057 & 77.57 & 22.43 & 74.14 & 25.86 & \\
\hline \multirow{2}{*}{\multicolumn{2}{|c|}{ Recipe 3A Mean }} & 83.9519 & & & & 77.70 & & 73.44 & 26.56 & 40.8 \\
\hline & & & & & & 0.51 & & 2.13 & 2.13 & 2.13 \\
\hline $3 \mathrm{~B}$ & $T 10$ & 88.3311 & 93.8732 & 89.7214 & 88.7173 & 74.91 & 25.09 & 72.22 & 27.78 & \\
\hline $3 B$ & T11 & 87.8981 & 94.1105 & 89.4547 & 88.3216 & 74.94 & 25.06 & 72.79 & 27.21 & \\
\hline $3 \mathrm{~B}$ & $T 12$ & 88.2013 & 94.8810 & 89.8887 & 88.6770 & 74.74 & 25.26 & 71.81 & 28.19 & \\
\hline \multicolumn{2}{|c|}{ Recipe 3B Mean } & & & & & 74.87 & & 72.27 & 27.73 & 40.2 \\
\hline \multicolumn{2}{|c|}{ Recipe 3B St. Dev. } & & & & & 0.11 & & 0.49 & $\frac{0.49 \mid}{21.94 \mid}$ & \\
\hline $4 \mathrm{~A}$ & $T 1$ & 87.7770 & 97.1317 & 90.2060 & 88.3100 & 74.03 & 25.97 & 78.06 & 21.94 & \\
\hline $4 \mathrm{~A}$ & $T 2$ & 88.1471 & 93.4115 & 89.5366 & 88.4424 & 73.61 & 26.39 & 78.75 & 21.25 & \\
\hline $4 \mathrm{~A}$ & T3 & 87.3307 & 93.6704 & 89.0320 & 87.6971 & 73.16 & 26.84 & 78.46 & $\frac{21.54}{21.58}$ & \\
\hline \multirow{2}{*}{\multicolumn{2}{|c|}{ Recipe 4A Mean }} & & & & & 73.60 & & 78.42 & 21.58 & 43.6 \\
\hline & & & & & & 0.44 & & 0.35 & $\frac{0.35}{30.31}$ & 0.35 \\
\hline $4 \mathrm{~B}$ & $1 \quad T 5$ & 88.6208 & 94.3447 & 90.0759 & 88.9164 & 74.58 & 25.42 & 79.69 & 20.31 & \\
\hline $4 \mathrm{~B}$ & T6 & 88.2214 & 94.9086 & 89.9418 & 88.5630 & 74.27 & 25.73 & 80.14 & 19.86 & \\
\hline $4 \mathrm{~B}$ & T8 & 88.0391 & 94.4247 & 89.7005 & 88.3643 & 73.98 & 26.02 & 80.43 & 19.57 & \\
\hline \multirow{2}{*}{\multicolumn{2}{|c|}{\begin{tabular}{|l} 
Recipe 4B Mean \\
Recipe 4B St. Dev.
\end{tabular}}} & & & & & \begin{tabular}{r|}
74.28 \\
0.30 \\
\end{tabular} & & \begin{tabular}{r|}
80.09 \\
0.37
\end{tabular} & $\frac{19.91}{0.37}$ & $\frac{44.5}{0.37}$ \\
\hline & & & & & & & & & & \\
\hline
\end{tabular}

Recipe 4B St. Dev.

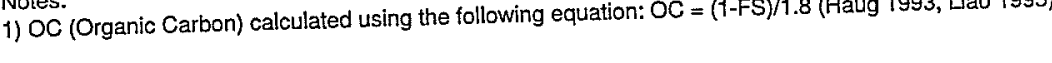


Phase 2 - Weekly Solids Analysis

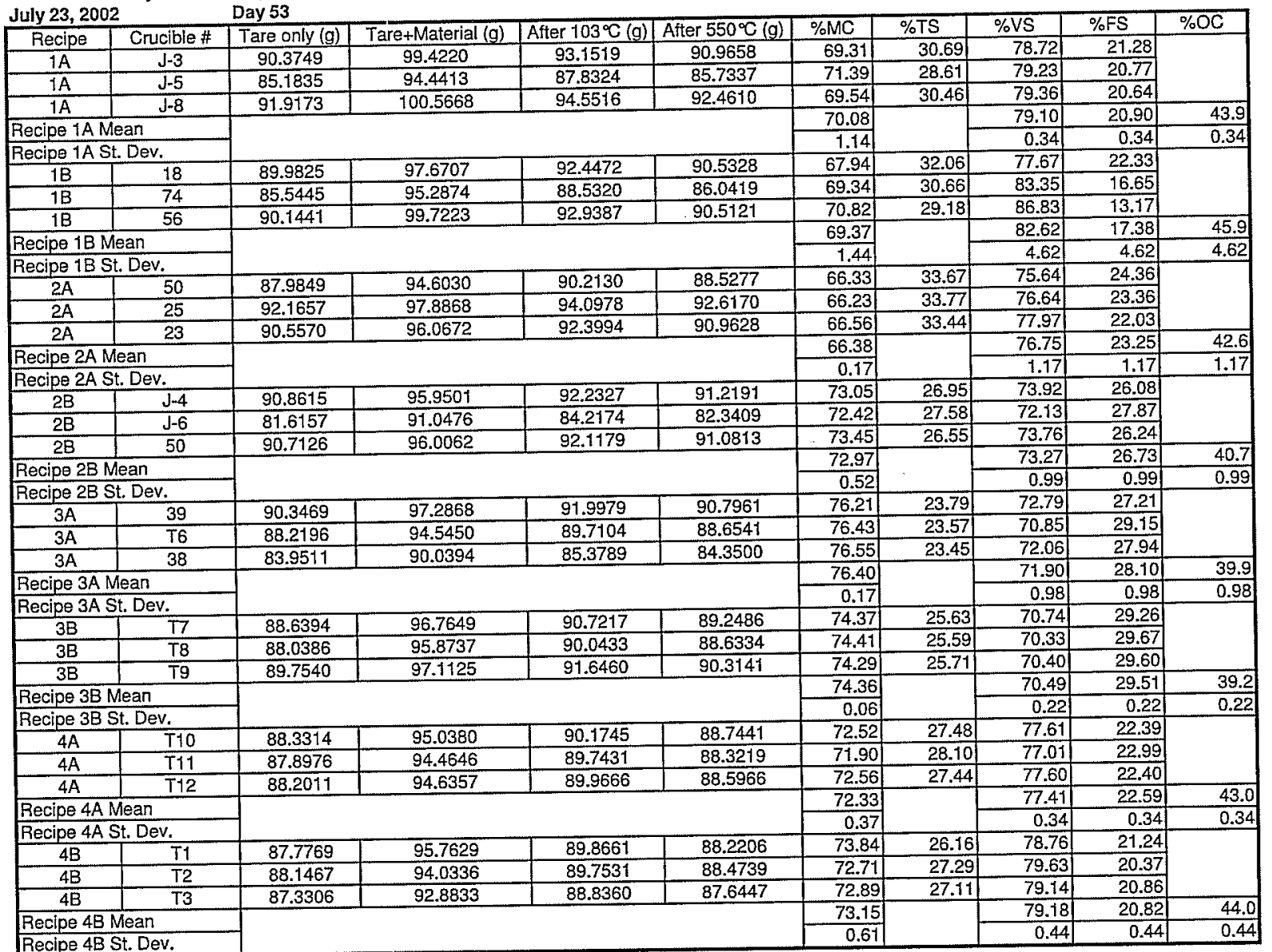

Notes:

Notes:
1) OC (Organic Carbon) calculated using the following equation: OC = (1-FS)/1.8 (Haug 1993, Liao 1995), where OC and FS are based on the dry weight fri 


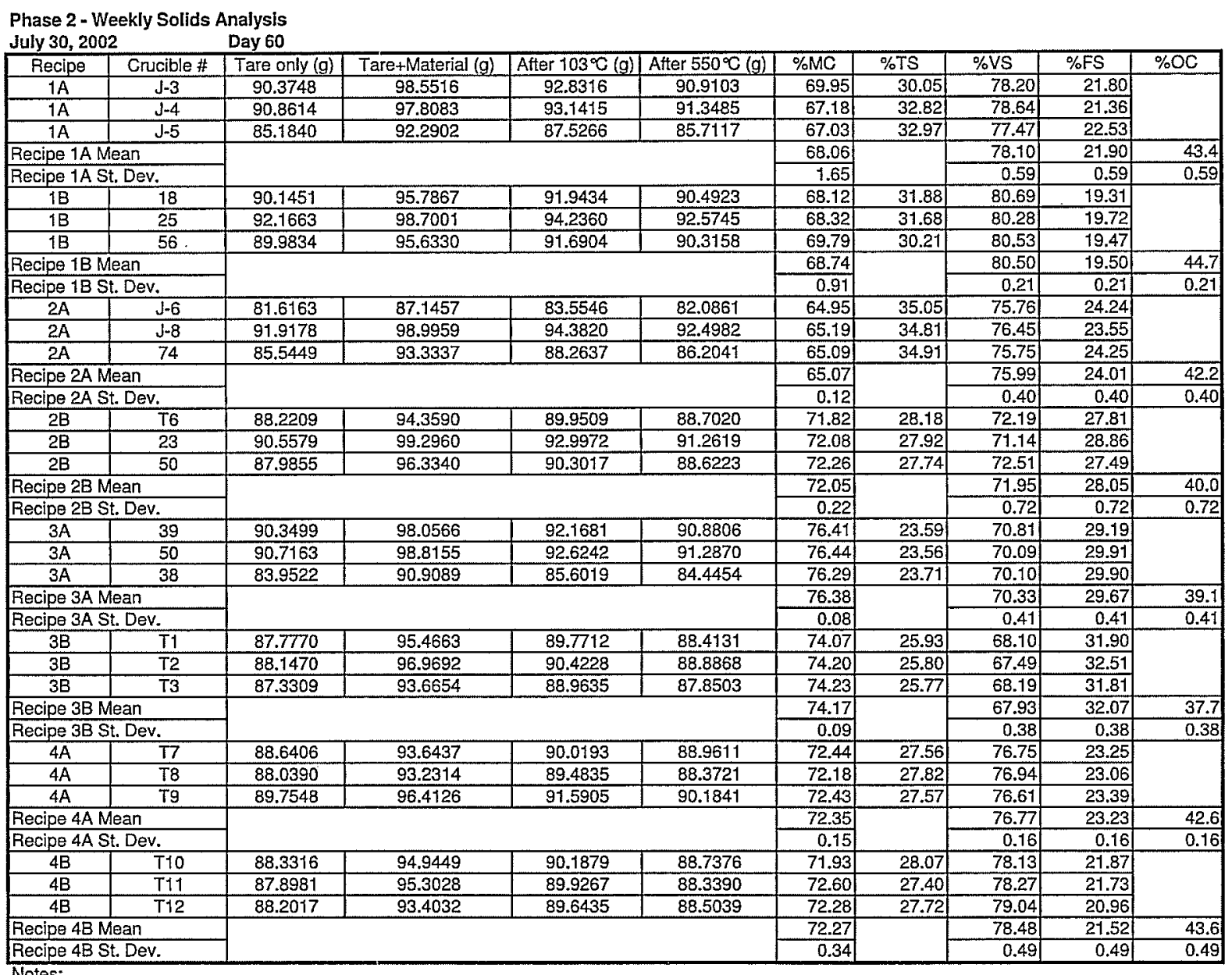

Notes:

1) $O C$ (Organic Carbon) calculated using the following equation: $O C=(1-F S) / 1.8$ (Haug 1993, Liao 1995), where OC and FS are based on the dry weight fri 
Phase 2 - Weekly Solids Analysis

\begin{tabular}{|c|c|c|c|c|c|c|c|c|c|c|}
\hline Recipe & Grucible \# & Tare only (g) & Tare+Material (g) & Affer $103^{\circ} \mathrm{C}(\mathrm{g})$ & After $550^{\circ} \mathrm{C}(\mathrm{g})$ & $\% M C$ & \%TS & \%VS & $\% \mathrm{FS}$ & $\% \mathrm{OC}$ \\
\hline $1 \mathrm{~A}$ & 25 & 92.1658 & 99.9656 & 94.5499 & 92.6828 & 69.43 & 30.57 & 78.31 & 21.69 & \\
\hline $1 \mathrm{~A}$ & 56 & 89.9831 & 98.2328 & 92.5080 & 90.5609 & 69.39 & 30.61 & 77.12 & 22.88 & \\
\hline $1 \mathrm{~A}$ & $\mathrm{~J}-6$ & 81.6157 & 89.5509 & 84.1334 & 82.1697 & 68.27 & 31.73 & 78.00 & 22.00 & \\
\hline \multirow{2}{*}{\multicolumn{2}{|c|}{$\begin{array}{l}\text { Recipe 1A Mean } \\
\text { Recipe 1A St Dev }\end{array}$}} & & & & & 69.03 & & 77.81 & 22.19 & 43.2 \\
\hline & & & & & & 0.66 & & 0.62 & 0.62 & 0.62 \\
\hline $1 \mathrm{~B}$ & 74 & 85.5433 & 93.6177 & 87.8401 & 86.0036 & 71.55 & 28.45 & 79.96 & 20.04 & \\
\hline $1 \mathrm{~B}$ & 50 & 87.9864 & 95.4055 & 90.1461 & 88.4099 & 70.89 & 29.11 & 80.39 & 19.61 & \\
\hline $1 \mathrm{~B}$ & 18 & 90.1443 & 99.3993 & 92.8422 & 90.6769 & 70.85 & 29.15 & 80.26 & 19.74 & \\
\hline \multicolumn{2}{|c|}{ Recipe 1B Mean } & & & & & 71.10 & & 80.20 & 19.80 & 44.6 \\
\hline \multicolumn{2}{|c|}{ Recipe 1B St. Dev. } & & & & & 0.40 & & 0.22 & 0.22 & 0.22 \\
\hline $2 A$ & J-3 & 90.3745 & 98.9259 & 92.9976 & 91.0494 & 69.33 & 30.67 & 74.27 & 25.73 & \\
\hline $2 \mathrm{~A}$ & $\sqrt{ }-4$ & 90.8610 & 96.5510 & 92.6237 & 91.3124 & 69.02 & 30.98 & 74.39 & 25.61 & \\
\hline $2 \mathrm{~A}$ & J-5 & 85.1832 & 90.7713 & 86.9549 & broke & 68.30 & 31.70 & & & \\
\hline \multicolumn{2}{|c|}{ Recipe 2A Mean } & & & & & 68.88 & & 74.33 & 25.67 & 41.3 \\
\hline \multicolumn{2}{|c|}{ Recipe 2A St. Dev. } & & & & & 0.53 & & 0.09 & 0.09 & 0.09 \\
\hline $2 \mathrm{~B}$ & 45 & 89.1909 & 95.3499 & 90.9460 & broke & 71.50 & 28.50 & & & \\
\hline $2 \mathrm{~B}$ & T6 & 88.2202 & 94.9901 & 90.1446 & 88.7915 & 71.57 & 28.43 & 70.31 & 29.69 & \\
\hline $2 \mathrm{~B}$ & $J-8$ & 91.9172 & 99.1940 & 94.0339 & 92.5374 & 70.91 & 29.09 & 70.70 & 29.30 & \\
\hline \multirow{2}{*}{\multicolumn{2}{|c|}{ Recipe 2B Mean }} & & & & & 71.33 & & 70.51 & 29.49 & 39.2 \\
\hline & Recipe 2B St. Dev. & & & & & 0.36 & & 0.27 & 0.27 & 0.27 \\
\hline $3 A$ & 50 & 90.7156 & 99.9752 & 93.0932 & 91.4753 & 74.32 & 25.68 & 68.05 & 31.95 & \\
\hline $3 A$ & 38 & 83.9518 & 91.4290 & 85.8246 & 84.5379 & 74.95 & 25.05 & 68.70 & 31.30 & \\
\hline $3 \mathrm{~A}$ & 39 & 90.3496 & 99.9806 & 92.8108 & 91.1532 & 74.45 & 25.55 & 67.35 & 32.65 & \\
\hline \multirow{2}{*}{\multicolumn{2}{|c|}{$\begin{array}{l}\text { Recipe 3A Mean } \\
\text { Recipe 3A St. Dev. }\end{array}$}} & & & & & 74.57 & & 68.03 & 31.97 & 37.8 \\
\hline & & & & & & 0.33 & & 0.68 & 0.68 & 0.68 \\
\hline $3 \mathrm{~B}$ & 77 & 88.6396 & 96.2309 & 90.6392 & 89.3128 & 73.66 & 26.34 & 66.33 & 33.67 & \\
\hline $3 \mathrm{~B}$ & T8 & 88.0385 & 96.4091 & 90.2662 & 88.7830 & 73.39 & 26.61 & 66.58 & 33.42 & \\
\hline $3 \mathrm{~B}$ & T9 & 89.7547 & 98.5890 & 92.1104 & 90.5702 & 73.33 & 26.67 & 65.38 & 34.62 & \\
\hline \multirow{2}{*}{\multicolumn{2}{|c|}{$\begin{array}{l}\text { Recipe 3B Mean } \\
\text { Recipe 3B St Dey }\end{array}$}} & & & & & 73.46 & & 66.10 & 33.90 & 36.7 \\
\hline Recipe 3B St. Dev. & & & & & & 0.17 & & 0.63 & 0.63 & 0.63 \\
\hline $4 A$ & $\mathrm{~T} 1$ & 87.7764 & 96.4778 & 90.2602 & 88.3973 & 71.46 & 28.54 & 75.00 & 25.00 & \\
\hline $4 \mathrm{~A}$ & T2 & 88.1465 & 96.1335 & 90.4136 & 88.6988 & 71.62 & 28.38 & 75.64 & 24.36 & \\
\hline $4 A$ & T3 & 87.3301 & 93.3857 & 89.0558 & 87.7559 & 71.50 & 28.50 & 75.33 & 24.67 & \\
\hline \multirow{2}{*}{\multicolumn{2}{|c|}{$\begin{array}{l}\text { Recipe 4A Mean } \\
\text { Recipe 4A St. Dev, }\end{array}$}} & & & & & 71.52 & & 75.32 & 24.68 & 41.8 \\
\hline & & & & & & 0.08 & & 0.32 & 0.32 & 0.32 \\
\hline $4 \mathrm{~B}$ & $T 10$ & 88.3309 & 96.6593 & 90.6409 & 88.8495 & 72.26 & 27.74 & 77.55 & 22.45 & \\
\hline 4B & $T 11$ & 87.8978 & 94.4343 & 89.7209 & 88.3019 & 72.11 & 27.89 & 77.83 & 22.17 & \\
\hline $4 \mathrm{~B}$ & $\mathrm{~T} 12$ & 88.2012 & 94.0967 & 89.8258 & 88.5585 & 72.44 & 27.56 & 78.011 & 21.99 & \\
\hline \multirow{2}{*}{\multicolumn{2}{|c|}{$\begin{array}{l}\text { Recipe 4B Mean } \\
\text { Recipe 4B St. Dev. }\end{array}$}} & & & & & 72.27 & & 77.80 & 22.20 & 43.2 \\
\hline & & & & & & 0.17 & & 0.23 & 0.23 & 0.23 \\
\hline
\end{tabular}

1) OC (Organic Carbon) calculated using the following equation: OC = (1-FS)/1.8 (Haug 1993, Liao 1995), where OC and FS are based on the dry weight fr 


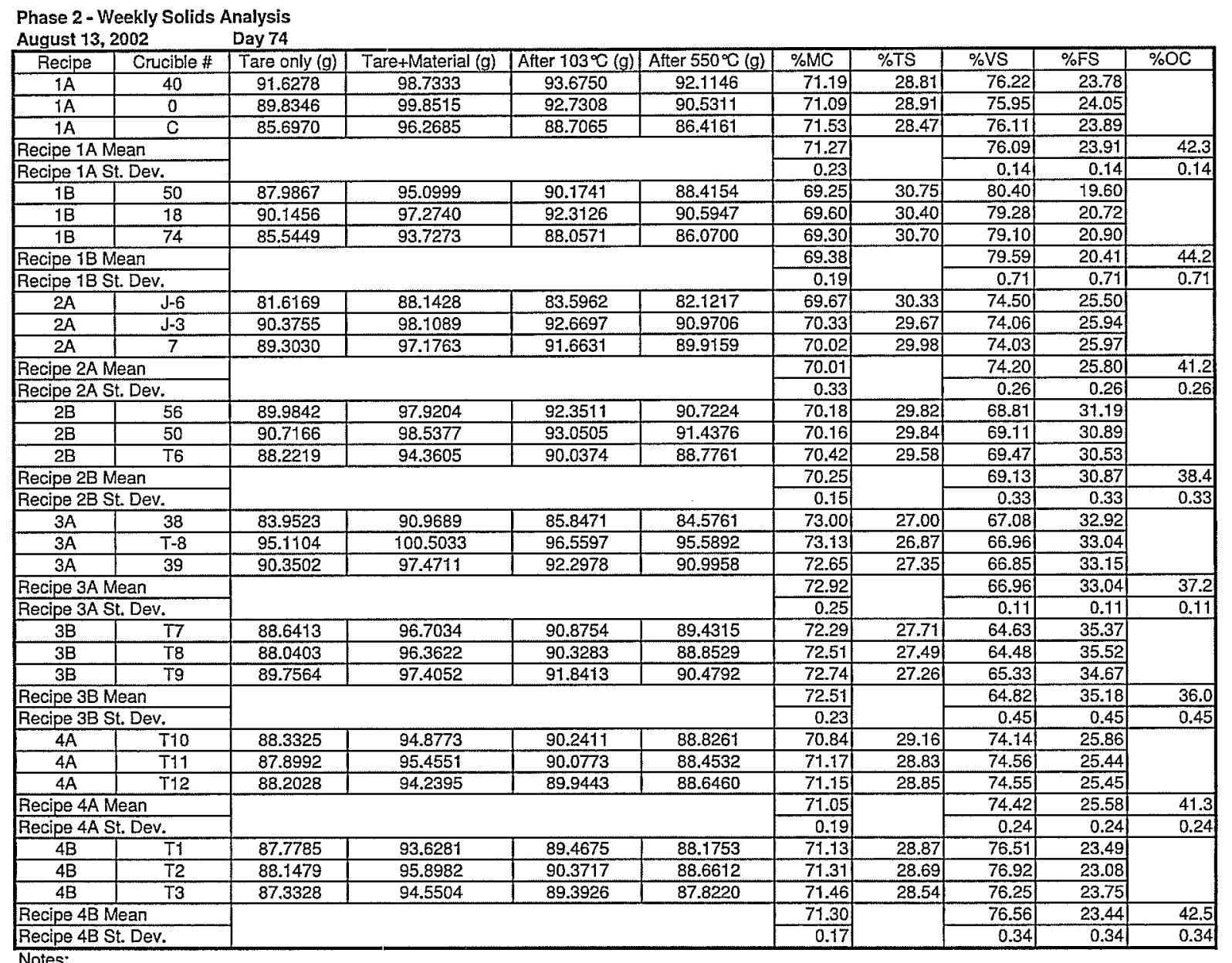

Notes:

1) OC (Organic Carbon) calculated using the following equation: OC = (1-FS)/1.8 (Haug 1993, Liao 1995), where OC and FS are based on the dry weight fri 
Phase 2 - Weekly Sollds Analysis

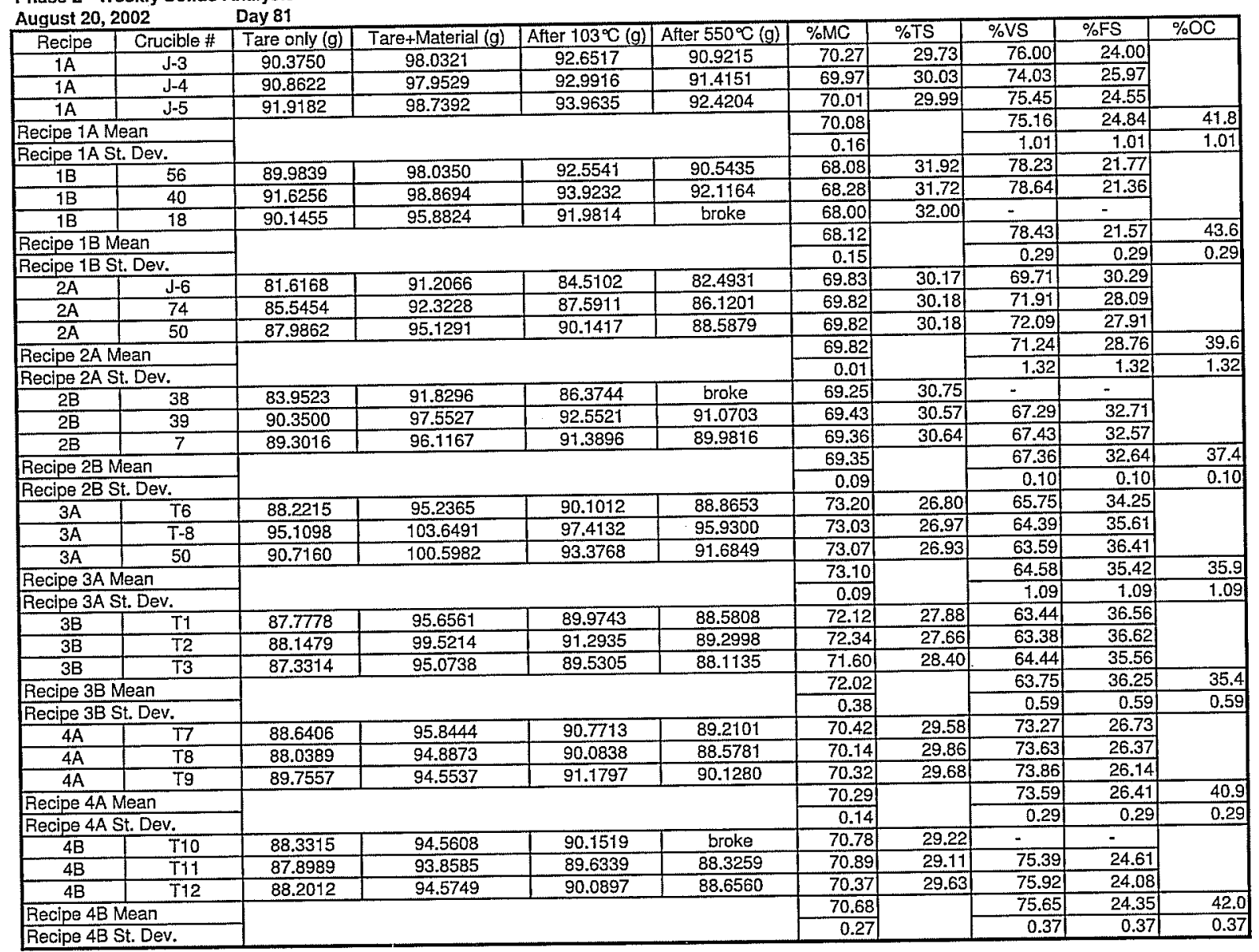

Recip:

1) OC (Organic Carbon) calculated using the following equation: OC = (1-FS)/1.8 (Haug 1993, Liao 1995), where OC and FS are based on the dry weight fri 


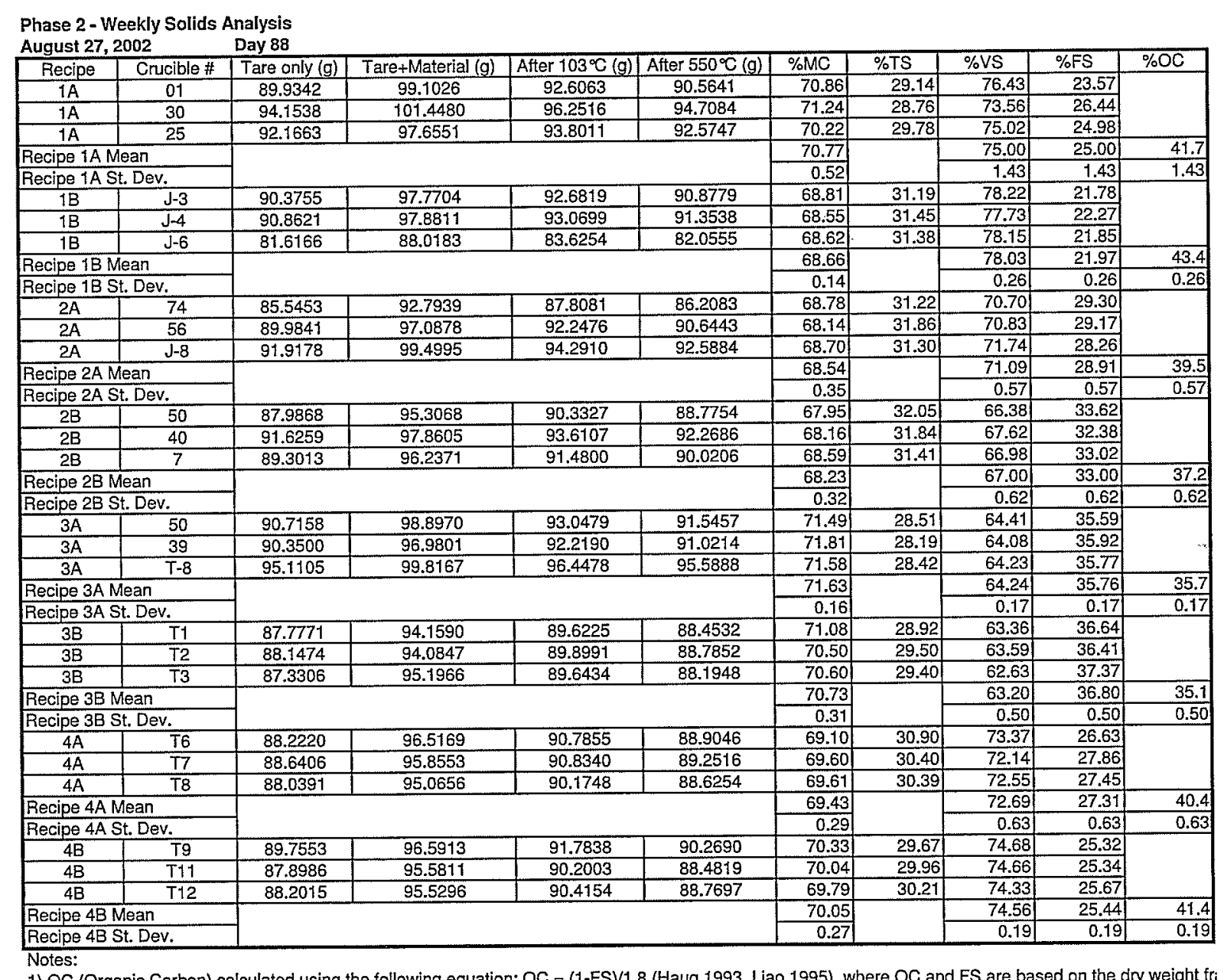


Phase 2 - Weekly Solids Analysis

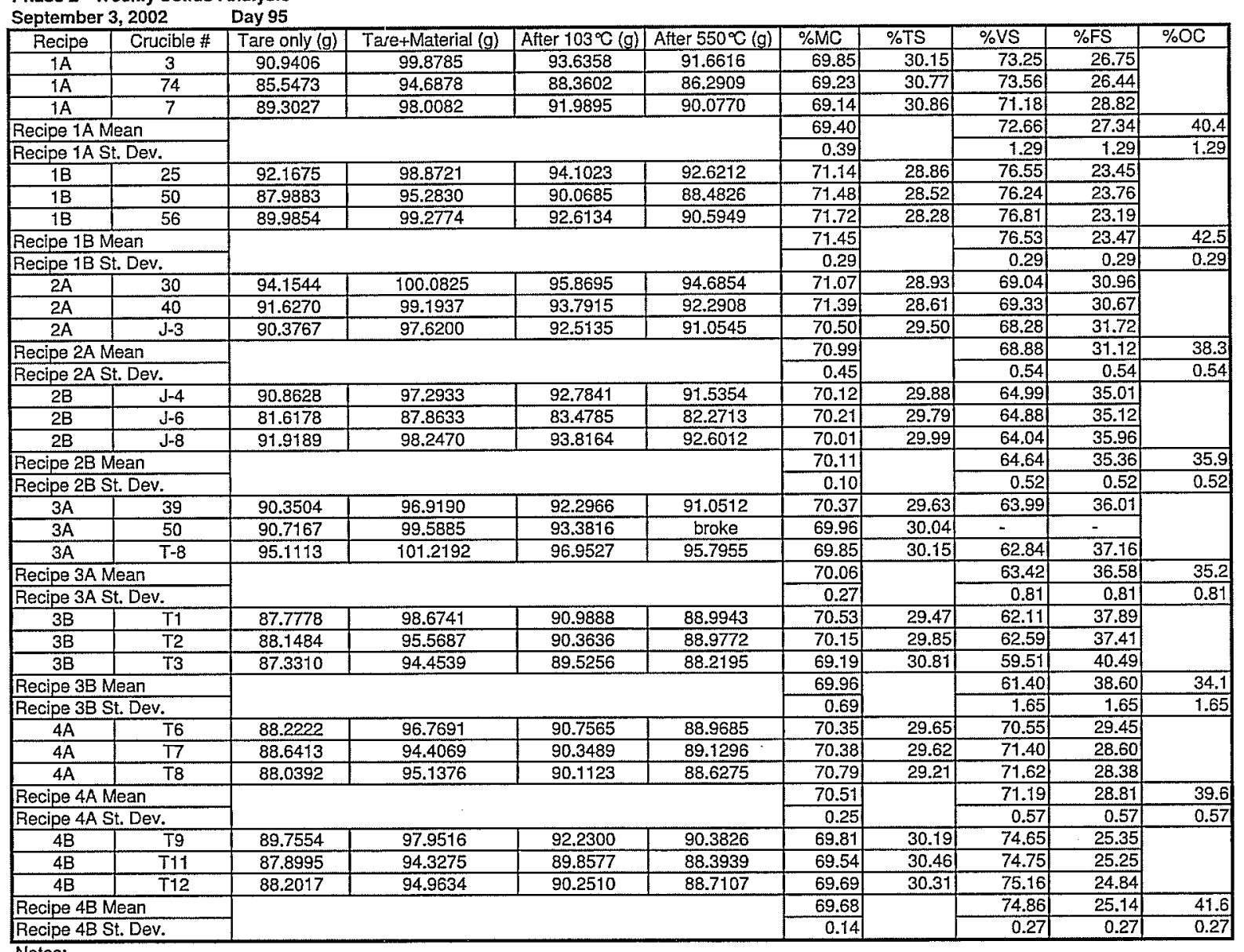

Notes:

1) $O C$ (Organic Carbon) calculated using the following equation: $O C=(1-F S) / 1.8$ (Haug 1993, Liao 1995), where OC and FS are based on the dry weight fn 
Phase 2 - Weekly Sollds Analysis

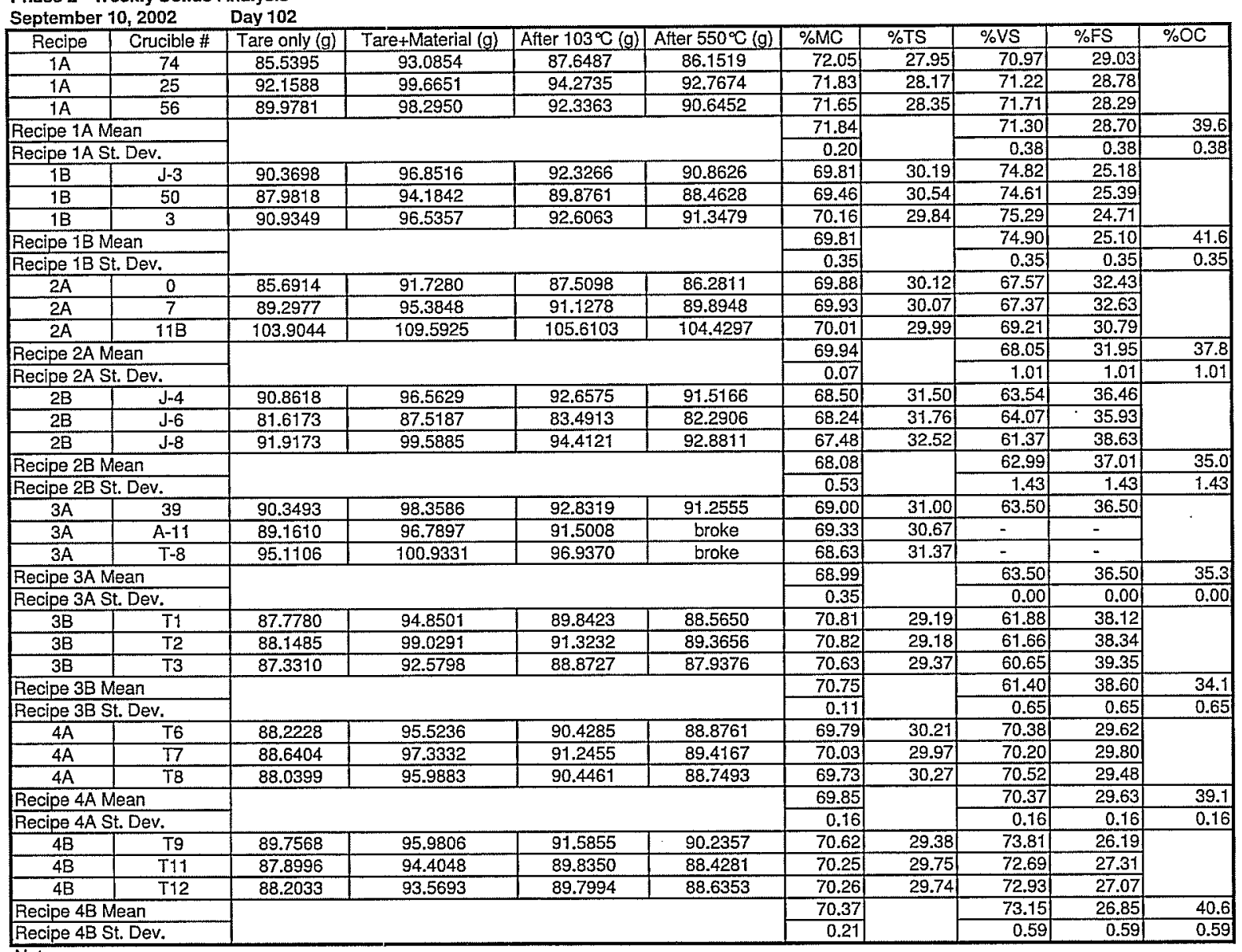

Notes:

1) OC (Organic Carbon) calculated using the following equation: $O C=(1-F S) / 1.8$ (Haug 1993, Liao 1995), where OC and FS are based on the dry weight fn 
Phase 2 - Weekly Solids Analysis

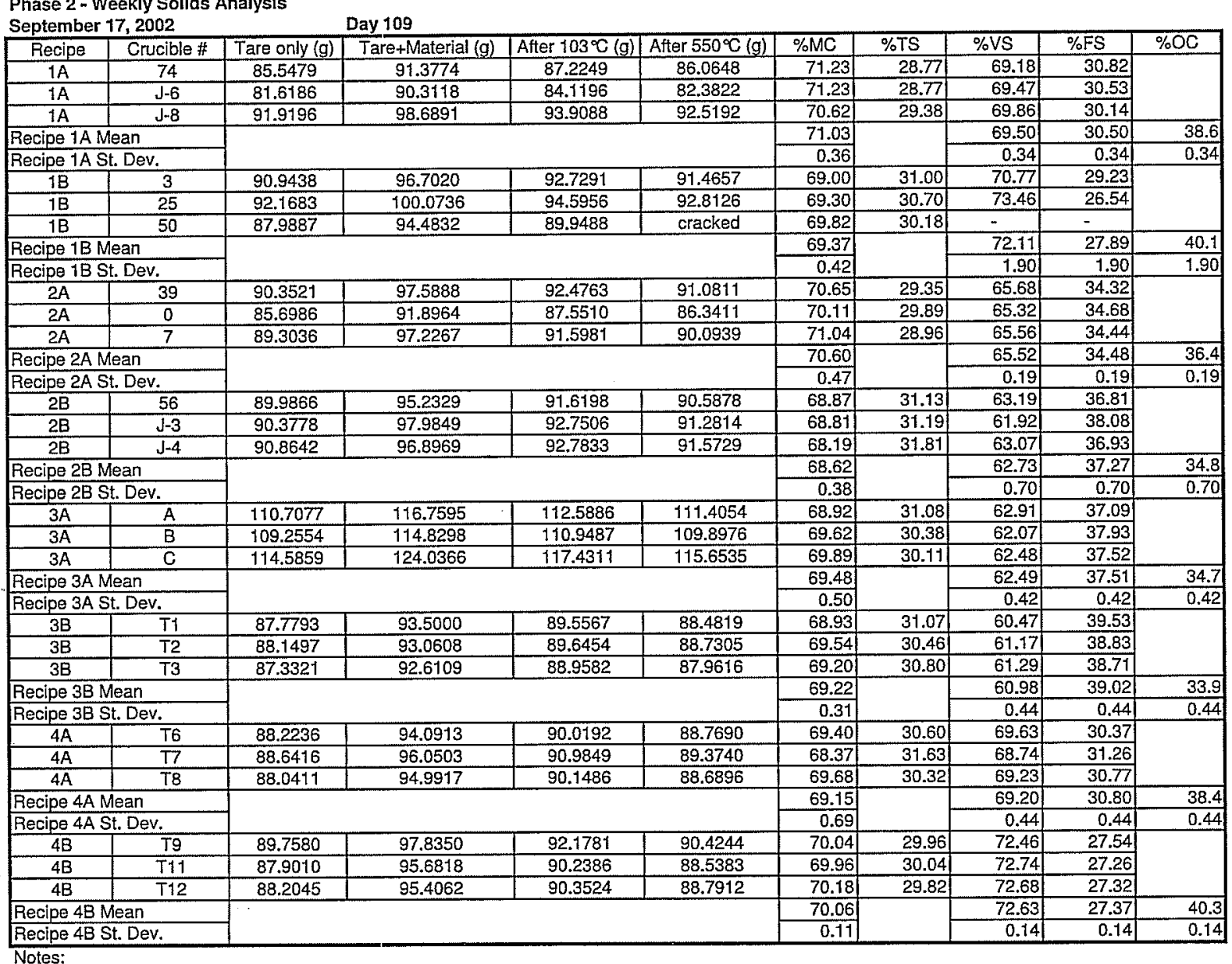

1) $\mathrm{OC}$ (Organic Carbon) calculated using the following equation: $\mathrm{OC}=(1-\mathrm{FS}) / 1.8$ (Haug 1993, Liao 1995), where $O C$ and $F S$ are based on the dry weight fri 
Phase 2 - Weekly Solids Analysis

\begin{tabular}{|c|c|c|c|c|c|c|c|c|c|c|}
\hline \multicolumn{3}{|c|}{ September 24,2002} & \multicolumn{8}{|l|}{ Day 116} \\
\hline Recipe & Crucible \# & Tare only $(\mathrm{g})$ & Tare+Material (g) & After $103^{\circ} \mathrm{C}(\mathrm{g})$ & After $550^{\circ} \mathrm{C}(\mathrm{g})$ & $\% \mathrm{MC}$ & \%TS & $\%$ VS & $\%$ FS & $\% \mathrm{OC}$ \\
\hline $1 \mathrm{~A}$ & $\mathrm{~J}-4$ & 90.8629 & 98.3716 & 93.1440 & 91.5855 & 69.62 & 30.38 & 68.32 & 31.68 & \\
\hline $1 \mathrm{~A}$ & $\mathrm{~J}-6$ & 81.6177 & 88.8015 & 83.8337 & 82.3388 & 69.15 & 30.85 & 67.46 & 32.54 & \\
\hline $1 \mathrm{~A}$ & $\mathrm{~J}-8$ & 91.9191 & 98.4106 & 93.9301 & 92.5797 & 69.02 & 30.98 & 67.15 & 32.85 & \\
\hline \multicolumn{2}{|c|}{ Recipe 1A Mean } & & & & & 69.26 & & 67.64 & 32.36 & 37.6 \\
\hline \multicolumn{2}{|c|}{ Recipe 1A St. Dev. } & & & & & 0.32 & & 0.61 & 0.61 & 0.61 \\
\hline 18 & 56 & 89.9859 & 98.0278 & 92.3236 & 90.6299 & 70.93 & 29.07 & 72.45 & 27.55 & \\
\hline $1 \mathrm{~B}$ & 74 & 85.5471 & 92.8983 & 87.7716 & 86.1523 & 69.74 & 30.26 & 72.79 & 27.21 & \\
\hline $1 \mathrm{~B}$ & 3 & 90.9428 & 98.5827 & 93.1688 & 91.5845 & 70.86 & 29.14 & 71.17 & 28.83 & \\
\hline \multicolumn{2}{|c|}{ Recipe 1B Mean } & & & & & 70.51 & & 72.14 & 27.86 & 40.1 \\
\hline \multicolumn{2}{|c|}{ Recipe 1B St. Dev. } & & & & & 0.67 & & 0.85 & 0.85 & 0.85 \\
\hline $2 A$ & 25 & 92.1671 & 100.3531 & 94.8430 & 93.2139 & 67.31 & 32.69 & 60.88 & 39.12 & \\
\hline $2 A$ & $\mathrm{~J}-3$ & 90.3768 & 97.6981 & 92.7131 & 91.2358 & 68.09 & 31.91 & 63.23 & 36.77 & \\
\hline $2 A$ & 0 & 85.6972 & 93.7825 & 88.2470 & 86.5990 & 68.46 & 31.54 & 64.63 & 35.37 & \\
\hline \multirow{2}{*}{\multicolumn{2}{|c|}{ Recipe 2A Mean }} & & & & & 67.95 & & 62.92 & 37.08 & 35.0 \\
\hline & & & & & & 0.59 & & 1.90 & 1.90 & 1.90 \\
\hline $2 B$ & A & 114.5826 & 120.3051 & 116.3284 & 115.2286 & 69.49 & 30.51 & 63.00 & 37.00 & \\
\hline $2 B$ & $\mathrm{~B}$ & 109.2520 & 115.8316 & 111.2832 & 110.0270 & 69.13 & 30.87 & 61.85 & 38.15 & \\
\hline $2 \mathrm{~B}$ & $\mathrm{C}$ & 110.7048 & 117.8296 & 112.9801 & 111.5611 & 68.07 & 31.93 & 62.37 & 37.63 & \\
\hline \multirow{2}{*}{\multicolumn{2}{|c|}{$\begin{array}{l}\text { Recipe 2B Mean } \\
\text { Recipe 2B St Dev }\end{array}$}} & & & & & 68.90 & & 62.40 & 37.60 & 34.7 \\
\hline & & & & & & 0.74 & & 0.58 & 0.58 & 0.58 \\
\hline $3 \mathrm{~A}$ & $\mathrm{D}$ & 110.4153 & 115.9318 & 112.0956 & 111.0683 & 69.54 & 30.46 & 61.14 & 38.86 & \\
\hline $3 A$ & 39 & 90.3513 & 97.3348 & 92.5518 & 91.1989 & 68.49 & 31.51 & 61.48 & 38.52 & \\
\hline $3 A$ & 7 & 89.3023 & 95.7415 & 91.2932 & 90.0612 & 69.08 & 30.92 & 61.88 & 38.12 & \\
\hline \multirow{2}{*}{\multicolumn{2}{|c|}{$\begin{array}{l}\text { Recipe 3A Mean } \\
\text { Recipe 3A St. Dev. }\end{array}$}} & & & & & 69.04 & & 61.50 & 38.50 & 34.2 \\
\hline & & & & & & 0.53 & & 0.37 & 0.37 & 0.37 \\
\hline $3 B$ & $\mathrm{~T} 1$ & 87.7781 & 96.6844 & 90.4275 & 88.8481 & 70.25 & 29.75 & 59.61 & 40.39 & \\
\hline 3B & $\mathrm{T} 2$ & 88.1483 & 95.1027 & 90.2062 & 88.9791 & 70.41 & 29.59 & 59.63 & 40.37 & \\
\hline $3 \mathrm{~B}$ & T3 & 87.3313 & 94.0860 & 89.3337 & 88.1371 & 70.36 & 29.64 & 59.76 & 40.24 & \\
\hline \multirow{2}{*}{\multicolumn{2}{|c|}{$\begin{array}{l}\text { Recipe 3B Mean } \\
\text { Recipe 3B St. Dev. }\end{array}$}} & & & & & 70.34 & & 59.67 & 40.33 & 33.1 \\
\hline & & & & & & 0.08 & & 0.08 & 0.08 & 0.08 \\
\hline $4 \mathrm{~A}$ & T6 & 88.2233 & 93.1351 & 89.6783 & 88.6873 & 70.38 & 29.62 & 68.11 & 31.89 & \\
\hline $4 \mathrm{~A}$ & $T 7$ & 88.6411 & 96.1672 & 90.8653 & 89.3598 & 70.45 & 29.55 & 67.69 & 32.31 & \\
\hline $4 \mathrm{~A}$ & T8 & 88.0406 & 94.7559 & 90.1467 & 88.7206 & 68.64 & $\begin{array}{l}31.36 \\
\end{array}$ & 67.71 & 32.29 & \\
\hline \multirow{2}{*}{\multicolumn{2}{|c|}{$\begin{array}{l}\text { Recipe 4A Mean } \\
\text { Recipe 4A St. Dev. }\end{array}$}} & & & & & 69.82 & & 67.84 & 32.16 & 37.7 \\
\hline & Recipe 4A St. Dev. & & & & & 1.03 & & 0.24 & 0.24 & 0.24 \\
\hline $4 \mathrm{~B}$ & $T 9$ & 89.7577 & 97.3103 & 92.0039 & 90.4111 & 70.26 & 29.74 & 70.91 & 29.09 & \\
\hline $4 \mathrm{~B}$ & $T 11$ & 87.9001 & 93.8833 & 89.7276 & 88.4275 & 69.46 & 30.54 & 71.14 & 28.86 & \\
\hline $4 \mathrm{~B}$ & $\mathrm{~T} 12$ & 88.2038 & 97.9093 & 91.0955 & 89.0521 & 70.21 & 29.79 & 70.66 & 29.34 & \\
\hline \multicolumn{2}{|c|}{$\begin{array}{l}\text { Recipe 4B Mean } \\
\text { Recipe 4B St. Dev. }\end{array}$} & & & & & $\begin{array}{r}69.97 \\
0.45 \\
\end{array}$ & & \begin{tabular}{r|}
70.91 \\
0.24 \\
\end{tabular} & $\begin{array}{r}29.09 \\
0.24 \\
\end{array}$ & $\begin{array}{l}39.4 \\
0.24 \\
\end{array}$ \\
\hline
\end{tabular}

Notes:

1) OC (Organic Carbon) calculated using the following equation: $O C=(1-F S) / 1.8$ (Haug 1993, Liao 1995), where OC and FS are based on the dry weight fri 
Phase 2 - Weekly Solids Analysis

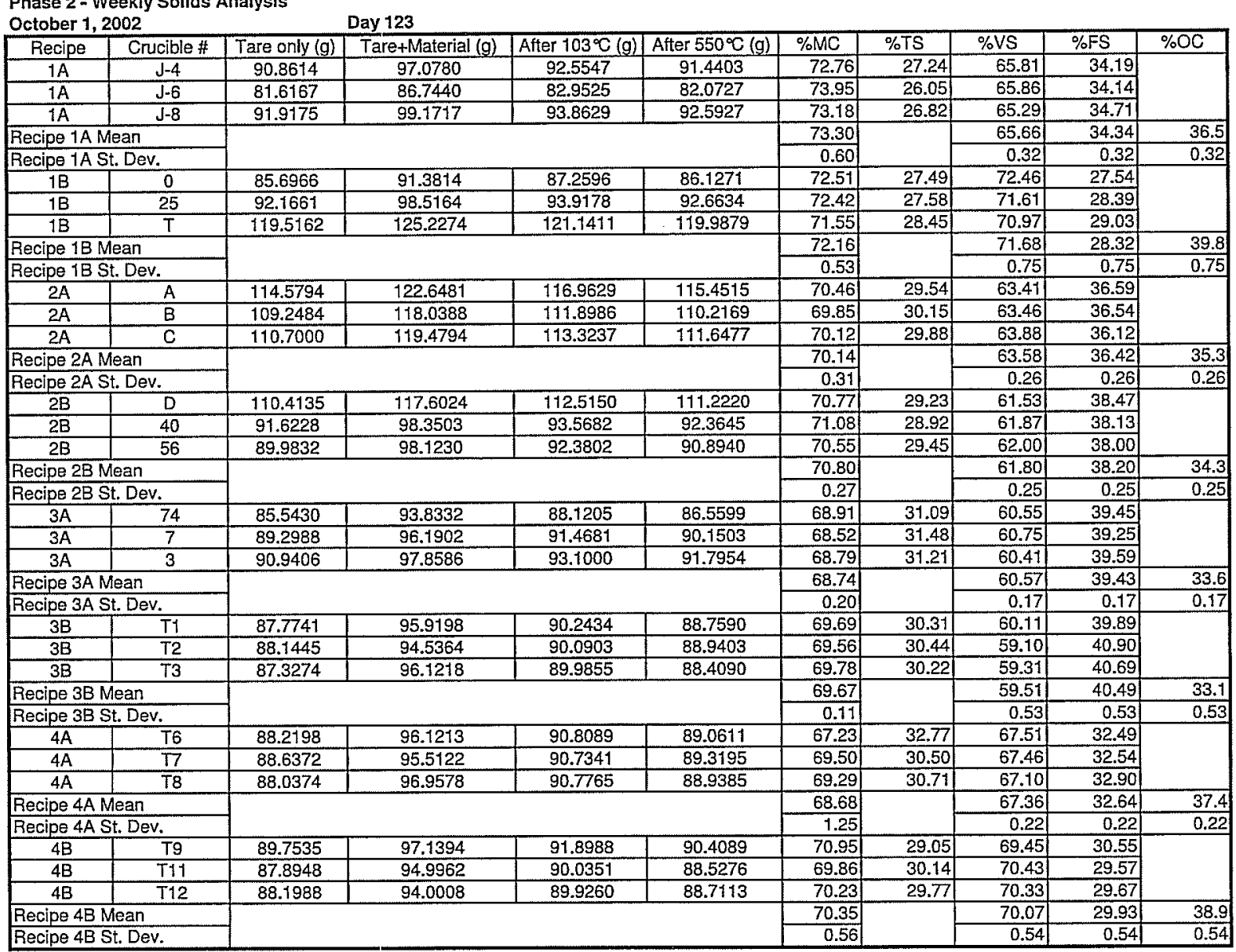

Notes:

1) OC (Organic Carbon) calculated using the following equation: OC = (1-FS)/1.8 (Haug 1993, Liao 1995), where OC and FS are based on the dry weight fr: 


\begin{tabular}{|c|c|c|c|c|c|c|c|c|c|c|}
\hline October 8 & & & Day 130 & & & & & & & \\
\hline$\frac{\text { Recipe }}{1 \mathrm{~A}}$ & Crucible \# & Tare only (g) & Tare+Material (g) & After $103^{\circ} \mathrm{C}(\mathrm{g})$ & After $550^{\circ} \mathrm{C}$ (g) & $\% M C$ & $\frac{\% T S}{3631}$ & $\frac{\% V S}{63,30}$ & $\frac{\%+5}{36,70}$ & $\%$ OC \\
\hline$\frac{1 \mathrm{~A}}{1 \mathrm{~A}}$ & $J-4$ & 90.8639 & 99.5728 & 94.0262 & 92.0244 & 63.69 & 36.31 & 63.30 & 36.70 & \\
\hline$\frac{1 A}{1 A}$ & $J-6$ & 81.6189 & 87.7898 & 83.6599 & 82.3550 & 66.93 & 33.07 & 63.93 & 36.07 & \\
\hline $1 A$ & $J-8$ & 91.9198 & 99.9437 & 94.5464 & 92.8920 & 67.27 & 32.73 & 62.99 & 37.01 & \\
\hline \multirow{2}{*}{\multicolumn{2}{|c|}{$\begin{array}{l}\text { Recipe 1A Mean } \\
\text { Recipe 1A St. Dev. }\end{array}$}} & & & & & 67.10 & & 63.41 & 36.59 & 35.2 \\
\hline & & & & & & 0.24 & & 0.48 & 0.48 & 0.48 \\
\hline $1 \mathrm{~B}$ & 7 & 89.3035 & 96.0917 & 91.4689 & 89.9945 & 68.10 & 31.90 & 68.09 & 31.91 & \\
\hline $1 \mathrm{~B}$ & 3 & 90.9441 & 97.3444 & 92.9330 & 91.5653 & 68.92 & 31.08 & 68.77 & 31,23 & \\
\hline IB & 40 & 91.6271 & 97.8026 & 93.6396 & 92.2321 & 67.41 & 32.59 & 69.94 & 30.06 & \\
\hline \multicolumn{2}{|c|}{$\frac{\text { Recipe 1B Mean }}{\text { Recipe 1B St. Dev. }}$} & & & & & 68.15 & & 68.93 & 31.07 & 38.3 \\
\hline \multicolumn{2}{|c|}{ Recipe 1B St. Dev. } & & & & & 0.76 & & 0.94 & 0.94 & 0.94 \\
\hline $2 \mathrm{~A}$ & $A$ & 114.5814 & 120.0638 & 116.2542 & 115.2161 & 69.49 & 30.51 & 62.06 & 37.94 & \\
\hline $2 \mathrm{~A}$ & $\mathrm{~B}$ & 109.2512 & 117.2396 & 111.6876 & 110.1712 & 69.50 & 30.50 & 62.24 & 37.76 & \\
\hline $2 A$ & C & 110.7038 & 117.8151 & 112.8418 & 111.5150 & 69.94 & 30.06 & 62.06 & 37.94 & \\
\hline \multicolumn{2}{|c|}{$\begin{array}{l}\text { Recipe 2A Mean } \\
\text { Recine 2A St Dey }\end{array}$} & & & & & 69.64 & & 62.12 & 37.88 & 34.5 \\
\hline \multicolumn{2}{|c|}{ Recipe 2A St. Dev. } & & & & & 0.25 & & 0.10 & 0.10 & 0.10 \\
\hline $2 \mathrm{~B}$ & $D$ & 110.4171 & 116.8490 & 112.5490 & 111.2296 & 66.85 & 33.15 & 61.89 & 38.11 & \\
\hline $2 \mathrm{~B}$ & $T$ & 119.5203 & 127.9496 & 122.2350 & 120.5789 & 67.79 & 32.21 & 61.00 & 39.00 & \\
\hline $2 \mathrm{~B}$ & 0 & 85.6989 & 93.5116 & 88.1938 & 86.6700 & 68.07 & 31.93 & 61.08 & 38.92 & \\
\hline \multirow{2}{*}{\multicolumn{2}{|c|}{ Recipe 2B Mean }} & & & & & 67.57 & & 61.32 & 38.68 & 34.7 \\
\hline & Recipe 2B St. Dev. & & & & & 0.64 & & 0.49 & 0.49 & 0.49 \\
\hline $3 A$ & 56 & 89.9868 & 96.7619 & 92.0416 & 90.8009 & 69.67 & 30,33 & 60.38 & 39.62 & \\
\hline $3 A$ & 25 & 92.1683 & 99.8066 & 94.5614 & 93.1515 & 68.67 & 31.33 & 58.92 & 41.08 & \\
\hline $3 A$ & 74 & 85.5479 & 92.2751 & 87.6458 & 86.3852 & 68.81 & 31.19 & 60.09 & 39.91 & \\
\hline \multirow{2}{*}{\multicolumn{2}{|c|}{$\begin{array}{l}\text { Recipe 3A Mean } \\
\text { Recipe 3A St. Dev. }\end{array}$}} & & & & & 69.05 & & 59.79 & 40.21 & 33.2 \\
\hline & & & & & & 0.54 & & 0.78 & 0.78 & 0.78 \\
\hline 3B & $\mathrm{Tt}$ & 87.7794 & 94.3401 & 89.7450 & 88.5859 & 70.04 & 29.96 & 58.97 & 41.03 & \\
\hline $3 \mathrm{~B}$ & T2 & 88.1493 & 95.3340 & 3173 & 89.0464 & 69.82 & 30.18 & 58.62 & 41.38 & \\
\hline $3 \mathrm{~B}$ & T3 & 87.3324 & 97.0156 & 90.2477 & 88.5325 & 69.89 & 30.11 & 58.83 & 41.17 & \\
\hline \multicolumn{2}{|c|}{ Recipe 3B Mean } & & & & & 69.92 & & 58.81 & 41.19 & 32.7 \\
\hline \multicolumn{2}{|c|}{ Recipe 3B St. Dev. } & & & & & 0.11 & & 0.18 & 0.18 & 0.18 \\
\hline $4 A$ & T6 & 88.2238 & 94.6027 & 90.0943 & 88.8541 & 70.68 & 29.32 & 66.30 & 33.70 & \\
\hline $4 \mathrm{~A}$ & 77 & 88.6408 & 94.9793 & 90.5241 & 89.2735 & 70.29 & 29.71 & 66.40 & 33.60 & \\
\hline $4 \mathrm{~A}$ & T8 & 88.0411 & 94.4133 & 89.8887 & 88.6561 & 71.01 & 28.99 & 66.71 & 33.29 & \\
\hline \multirow{2}{*}{\multicolumn{2}{|c|}{$\begin{array}{l}\text { Recipe 4A Mean } \\
\text { Recipe 4A St. Dev. } \\
\end{array}$}} & & & & & 70.66 & & 66.47 & 33.53 & 36.9 \\
\hline & & & & & & 0.36 & & 0.21 & 0.21 & 0.21 \\
\hline \begin{tabular}{|l|}
$4 \mathrm{~B}$ \\
\end{tabular} & $\mathrm{Tg}$ & 89.7588 & 94.8787 & 91.2977 & 90.2338 & 69.94 & 30.06 & 69.13 & 30.87 & \\
\hline $4 \mathrm{~B}$ & $T 11$ & 87.9013 & 97.6580 & 90.7258 & 88.7860 & 71.05 & 28.95 & 68.68 & 31.32 & \\
\hline 4B & $\mathrm{T} 12$ & 88.2047 & 94.9315 & 90.2290 & 88.8364 & 69.91 & 30.09 & 68.79 & 31.21 & \\
\hline \multirow{2}{*}{\multicolumn{2}{|c|}{\begin{tabular}{|l} 
Recipe 4B Mean \\
Recipe 4B St. Dev. \\
\end{tabular}}} & & & & & $\frac{70.30}{0.65}$ & & $\frac{68.87}{0.24}$ & $\begin{array}{r}31.13 \\
0.24\end{array}$ & $\begin{array}{l}38.3 \\
0.24 \\
\end{array}$ \\
\hline & & & & & & $0.65]$ & & & & 0.24 \\
\hline
\end{tabular}

Notes:

1) $\mathrm{OC}$ (Organic Carbon) calculated using the following equation: $O C=(1-F S) / 1.8$ (Haug 1993, Liao 1995), where OC and FS are based on the dry weight fr 
Phase 2 - Weekly Solids Analysis

October 15, 2002 Day 137

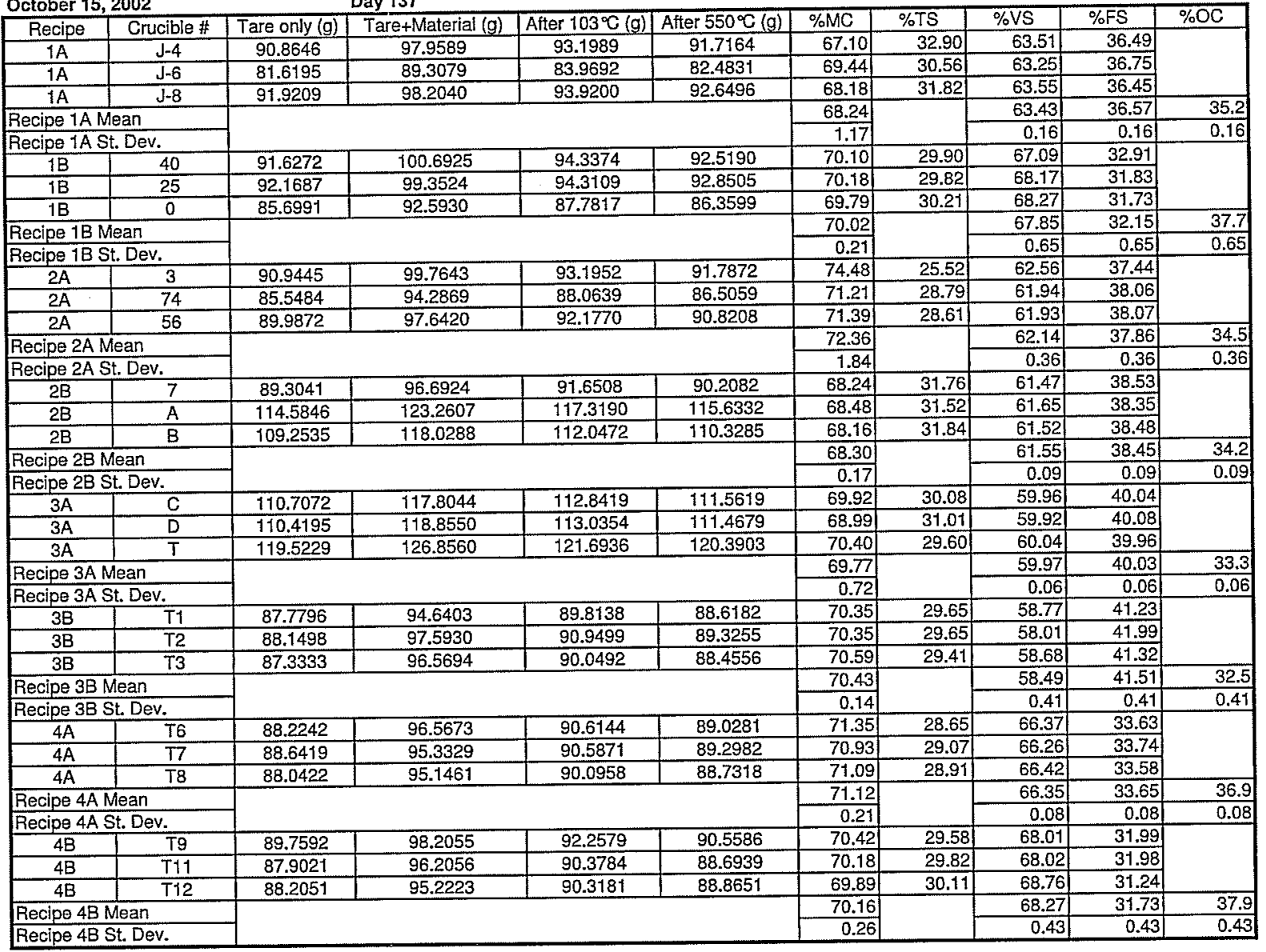

\section{Recipe 4B St. Dev.}

1) OC (Organic Carbon) calculated using the following equation: OC = (1-FS)/1.8 (Haug 1993, Liao 1995), where OC and FS are based on the dry weight fri 
Phase 2 - Weekly Solids Analysis

October 22, 2002

Day 144

\begin{tabular}{|c|c|c|c|c|c|c|c|c|c|c|}
\hline Recipe & Crucible \# & Tare only (g) & Tare+Material (g) & After $103^{\circ} \mathrm{C}(\mathrm{g})$ & After $550^{\circ} \mathrm{C}(\mathrm{g})$ & $\% \mathrm{MC}$ & \%TS & $\%$ VS & $\%$ FS & $\% \mathrm{OC}$ \\
\hline $1 \mathrm{~A}$ & $J-4$ & 90.8646 & 98.7277 & 92.8942 & 91.6213 & 74.19 & 25.81 & 62.72 & 37.28 & \\
\hline TA & $J-6$ & 81.6194 & 89.4976 & 83.8640 & 82.5271 & 71.51 & 28.49 & 59.56 & 40.44 & \\
\hline $1 \mathrm{~A}$ & $\sqrt{-8}$ & 91.9202 & 100.7963 & 94.2895 & 92.8126 & 73.31 & 26.69 & 62.33 & 37.67 & \\
\hline \multirow{2}{*}{\multicolumn{2}{|c|}{$\begin{array}{l}\text { Recipe 1A Mean } \\
\text { Recioe 1A St. Dev. }\end{array}$}} & & & & & 73.00 & & 61.54 & 38.46 & 34.2 \\
\hline & & & & & & 1.37 & & 1.72 & 1.72 & 1.72 \\
\hline $1 \mathrm{~B}$ & 56 & 89.9871 & 97.6724 & 92.1695 & 90.7471 & 71.60 & 28.40 & 65.18 & 34.82 & \\
\hline $1 \mathrm{~B}$ & 25 & 92.1689 & 98.8822 & 94.0874 & 92.8138 & 71.42 & 28.58 & 66.39 & 33.61 & \\
\hline $1 B$ & 0 & 85.6993 & 91.6026 & 87.4117 & 86.2879 & 70.99 & 29.01 & 65.63 & 34.37 & \\
\hline \multicolumn{2}{|c|}{ Recipe 1B Mean } & & & & & 71.34 & & 65.73 & 34.27 & 36.5 \\
\hline \multicolumn{2}{|c|}{ Recipe 1B St. Dev. } & & & & & 0.31 & & 0.61 & 0.61 & 0.61 \\
\hline $2 A$ & 40 & 91.6273 & 97.6813 & 93.4647 & 92.3277 & 69.65 & 30.35 & 61.88 & 38.12 & \\
\hline $2 \mathrm{~A}$ & 3 & 90.9442 & 99.7856 & 93.6087 & 91.9700 & 69.86 & 30.14 & 61.50 & 38.50 & \\
\hline $2 \mathrm{~A}$ & 74 & 85.5482 & 93.0263 & 87.8294 & 86.4166 & 69.49 & 30.51 & 61.93 & 38.07 & \\
\hline \multirow{2}{*}{\multicolumn{2}{|c|}{$\begin{array}{l}\text { Recipe 2A Mean } \\
\text { Recipe 2A St. Dev. }\end{array}$}} & & & & & 69.67 & & 61.77 & 38.23 & 34.3 \\
\hline & & & & & & 0.18 & & 0.24 & 0.24 & 0.24 \\
\hline $2 \mathrm{~B}$ & A & 114.5843 & 122.3309 & 116.8107 & 115.4680 & 71.26 & 28.74 & 60.31 & 39.69 & \\
\hline $2 B$ & $B$ & 109.2534 & 116.3460 & 111.2883 & 110.0603 & 71.31 & 28.69 & 60.35 & 39.65 & \\
\hline $2 \mathrm{~B}$ & $\mathrm{C}$ & 110.7057 & 119.8955 & 113.3522 & 111.7563 & 71.20 & 28.80 & 60.30 & 39.70 & \\
\hline \multirow{2}{*}{\multicolumn{2}{|c|}{$\begin{array}{l}\text { Recipe 2B Mean } \\
\text { Recipe 2B St. Dev, }\end{array}$}} & & & & & 71.26 & & 60.32 & 39.68 & 33.5 \\
\hline & & & & & & 0.05 & & 0.02 & 0.02 & 0.02 \\
\hline $3 A$ & $\mathrm{D}$ & 110.4185 & 117.3305 & 112.4626 & 111.2490 & 70.43 & 29.57 & 59.37 & 40.63 & \\
\hline $3 A$ & $\bar{E}$ & 119.5226 & 129.0992 & 122.3618 & 120.6945 & 70.35 & 29.65 & 58.72 & 41.28 & \\
\hline $3 \mathrm{~A}$ & 7 & 89.3041 & 97.8563 & 91.8728 & 90.3876 & 69.96 & 30.04 & 57.82 & 42.18 & \\
\hline \multicolumn{2}{|c|}{ Recipe 3A Mean } & & & & & 70.25 & & 58.64 & 41.36 & 32.6 \\
\hline \multicolumn{2}{|c|}{ Recipe 3A St. Dev. } & & & & & 0.25 & & 0.78 & 0.78 & 0.78 \\
\hline $3 \mathrm{~B}$ & $\mathrm{~T} 1$ & 87.7793 & 94.6855 & 89.8715 & 88.6521 & 69.71 & 30.29 & 58.28 & 41.72 & \\
\hline $3 \mathrm{~B}$ & $\mathrm{T2}$ & 88.1496 & 95.9777 & 90.5316 & 89.1388 & 69.57 & 30.43 & 58.47 & 41.53 & \\
\hline $3 \mathrm{~B}$ & T3 & 87.3327 & 94.6231 & 89.5837 & 88.2960 & 69.12 & 30.88 & 57.21 & 42.79 & \\
\hline \multirow{2}{*}{\multicolumn{2}{|c|}{ Recipe 3B Mean }} & & & & & 69.47 & & 57.99 & 42.01 & 32.2 \\
\hline Recipe 3B St. Dev. & & & & & & 0.30 & & 0.68 & 0.68 & 0.68 \\
\hline $4 \mathrm{~A}$ & T6 & 88.2245 & 96.1098 & 90.5638 & 89.0295 & 70.33 & 29.67 & 65.59 & 34.41 & \\
\hline $4 \mathrm{~A}$ & $\pi 7$ & 88.6421 & 97.2160 & 91.2218 & 89.5363 & 69.91 & 30.09 & 65.34 & 34.66 & \\
\hline $4 \mathrm{~A}$ & $T 8$ & 88.0421 & 97.2594 & 90.7513 & 88.9810 & 70.61 & 29.39 & 65.34 & 34.66 & \\
\hline \multirow{2}{*}{\multicolumn{2}{|c|}{$\begin{array}{l}\text { Recipe 4A Mean } \\
\text { Recipe 4A St. Dev. }\end{array}$}} & & & & & 70.28 & & 65.42 & 34.58 & 36.3 \\
\hline & & & & & & 0.35 & & 0.14 & 0.14 & 0.14 \\
\hline $4 \mathrm{~B}$ & T9 & 89.7589 & 97.3903 & 91.9538 & 90.4819 & 71.24 & 28.76 & 67.06 & 32.94 & \\
\hline $4 \mathrm{~B}$ & $T 11$ & 87.9015 & $95.315 t$ & 90.0552 & 88.6227 & 70.95 & 29.05 & 66.51 & 33.49 & \\
\hline $4 \mathrm{~B}$ & $\mathrm{~T} 12$ & 88.2048 & 94.2541 & 89.9482 & 88.7834 & 71.18 & 28.82 & 66.81 & 33.19 & \\
\hline \multirow{2}{*}{\multicolumn{2}{|c|}{\begin{tabular}{|l} 
Recipe 4B Mean \\
Recipe 4B St. Dev.
\end{tabular}}} & & & & & 71.12 & & 66.80 & $\begin{array}{l}33.20 \\
0.77 \\
\end{array}$ & $\frac{37.1}{0.27}$ \\
\hline & & & & & & 0.15 & & 0.27 & 0.27 & 0.27 \\
\hline
\end{tabular}

Recipe 48 St Dev.

Notes:
1) OC (Organic Carbon) calculated using the following equation: $O C=(1-F S) / 1.8$ (Haug 1993, Liao 1995), where OC and FS are based on the dry weight fri 
Phase 2 - Weekly Solids Analysis

October 29, 2002

Day 151

\begin{tabular}{|c|c|c|c|c|c|c|c|c|c|c|}
\hline Recipe & Crucible \# & Tare only (g) & Tare+Material (g) & After $103^{\circ} \mathrm{C}(\mathrm{g})$ & After $550^{\circ} \mathrm{C}(\mathrm{g})$ & $\% M C$ & $\%$ TS & $\%$ VS & $\%$ FS & $\% O C$ \\
\hline $1 \mathrm{~A}$ & $J-4$ & 90.8662 & 98.8736 & 93.2702 & 91.7768 & 69.98 & 30.02 & 62.12 & 37.88 & \\
\hline $1 \mathrm{~A}$ & $J-6$ & 81.6205 & 88.7664 & 83.8163 & 82.4554 & 69.27 & 30.73 & 61.98 & 38.02 & \\
\hline $1 \mathrm{~A}$ & $J-8$ & 91.9214 & 100.0909 & 94.4666 & 92.8813 & 68.85 & 31.15 & 62.29 & 37.71 & \\
\hline \multirow{2}{*}{\multicolumn{2}{|c|}{$\begin{array}{l}\text { Recipe 1A Mean } \\
\text { Recipe 1A St. Dev. }\end{array}$}} & & & & & 69.36 & & 62.13 & 37.87 & 34.5 \\
\hline & & & & & & 0.57 & & 0.15 & 0.15 & 0.15 \\
\hline $1 B$ & 56 & 89.9885 & 97.7214 & 92.3223 & 90.8261 & 69.82 & 30.18 & 64.11 & 35.89 & \\
\hline TB & 74 & 85.5493 & 91.7073 & 87.3140 & 86.1727 & 71.34 & 28.66 & 64.67 & 35.33 & \\
\hline $1 \mathrm{~B}$ & 40 & 91.6288 & 99.2375 & 93.8618 & 92.4160 & 70.65 & 29.35 & 64.75 & 35.25 & \\
\hline \multicolumn{2}{|c|}{ Recipe 1B Mean } & & & & & 70.60 & & 64.51 & 35.49 & 35.8 \\
\hline \multicolumn{2}{|c|}{ Recipe 1B St. Dev. } & & & & & 0.76 & & 0.35 & 0.35 & 0.35 \\
\hline $2 A$ & 3 & 90.9456 & 99.4855 & 93.3528 & 91.8739 & 71.81 & 28.19 & 67.44 & 38.56 & \\
\hline $2 \mathrm{~A}$ & 25 & 92.1701 & 99.9440 & 94.3985 & 93.0370 & 71.33 & 28.67 & 61.10 & 38.90 & \\
\hline $2 A$ & 7 & 89.3049 & 97.1441 & 97.5422 & 90.1763 & 71.46 & 28.54 & 61.05 & 38.95 & \\
\hline \multicolumn{2}{|c|}{ Recipe 2A Mean } & & & & & 71.54 & & 61.20 & 38.80 & 34.0 \\
\hline \multicolumn{2}{|c|}{ Recipe 2A St. Dev. } & & & & & 0.25 & & 0.21 & 0.21 & 0.21 \\
\hline $2 B$ & A & 114.5881 & 122.6855 & 116.8747 & 115.5019 & 71.76 & 28.24 & 60.04 & 39.96 & \\
\hline $2 \mathrm{~B}$ & $\bar{B}$ & 109.2575 & 117.7518 & 111.6373 & 110.1955 & 71.98 & 28.02 & 60.58 & 39.42 & \\
\hline $2 B$ & $\mathrm{c}$ & 110.7104 & 120.4175 & 113.4571 & 111.8549 & 71.70 & 28.30 & 58.33 & 41.67 & \\
\hline \multirow{2}{*}{\multicolumn{2}{|c|}{$\begin{array}{l}\text { Recipe 2B Mean } \\
\text { Recipe 2B St. Dev. }\end{array}$}} & & & & & 71.82 & & 59.65 & 40.35 & 33.1 \\
\hline & & & & & & 0.15 & & 1.17 & 1.17 & 1.17 \\
\hline $3 \mathrm{~A}$ & $D$ & 110.4226 & 118.4799 & 112.7237 & 111.3775 & 71.44 & 28.56 & 58.50 & 41.50 & \\
\hline $3 A$ & $E$ & 119.5263 & 129.2505 & 122.3633 & 120.6859 & 70.83 & 29.17 & 59.13 & 40.87 & \\
\hline $3 \mathrm{~A}$ & 0 & 85.7006 & 94.9635 & 88.4049 & 86.8036 & 70.81 & 29.19 & 59.21 & 40.79 & \\
\hline \multirow{2}{*}{\multicolumn{2}{|c|}{$\begin{array}{l}\text { Recipe 3A Mean } \\
\text { Recipe 3A St. Dev. }\end{array}$}} & & & & & 71.02 & & 58.95 & 41.05 & 32.7 \\
\hline & & & & & & 0.36 & & 0.39 & 0.39 & 0.39 \\
\hline $3 \mathrm{~B}$ & $\mathrm{~T} 1$ & 87.7807 & 94.1208 & 89.6697 & 88.5737 & 70.21 & 29.79 & 58.02 & 41.98 & \\
\hline $3 B$ & $\mathrm{~T} 2$ & 88.1509 & 98.0143 & 91.0697 & 89.3675 & 70.41 & 29.59 & 58.32 & 41.68 & \\
\hline $3 \mathrm{~B}$ & T3 & 87.3338 & 95.7724 & 89.8223 & 88.3764 & 70.51 & 29.49 & 58.10 & 41.90 & \\
\hline \multirow{2}{*}{\multicolumn{2}{|c|}{$\begin{array}{l}\text { Recipe 3B Mean } \\
\text { Recipe 3B St. Dev }\end{array}$}} & & & & & 70.37 & & 58.15 & 41.85 & 32.3 \\
\hline & & & & & & 0.16 & & 0.15 & 0.15 & 0.15 \\
\hline $4 \mathrm{~A}$ & T6 & 88.2251 & 98.6120 & 91.2561 & 89.2854 & 70.82 & 29.18 & 65.02 & 34.98 & \\
\hline $4 \mathrm{~A}$ & $\pi$ & 88.6427 & 97.8568 & 91.3623 & 89.5928 & 70.48 & 29.52 & 65.06 & 34.94 & \\
\hline $4 A$ & $\mathrm{~T} 8$ & 88.0430 & 95.9965 & 90.3457 & 88.8508 & 71.05 & 28.95 & 64.92 & 35.08 & \\
\hline \multirow{2}{*}{\multicolumn{2}{|c|}{$\begin{array}{l}\text { Recipe 4A Mean } \\
\text { Recipe 4A St. Dev. }\end{array}$}} & & & & & 70.78 & & 65.00 & 35.00 & 36.1 \\
\hline & & & & & & 0.28 & & 0.07 & 0.07 & 0.07 \\
\hline $4 B$ & T9 & 89.7604 & 96.6280 & 91.8360 & 90.4543 & 69.78 & 30.22 & 66.57 & 33.43 & \\
\hline $4 \mathrm{~B}$ & $T 11$ & 87.9027 & 95.8407 & 90.3084 & 88.7305 & 69.69 & 30.31 & 65.59 & 34.41 & \\
\hline $4 \mathrm{~B}$ & $\mathrm{~T} 12$ & 88.2061 & 95.0893 & 90.2636 & 88.9016 & 70.11 & 29.89 & 66.20 & 33.80 & \\
\hline \multirow{2}{*}{\multicolumn{2}{|c|}{\begin{tabular}{|l} 
Recipe 4B Mean \\
Recipe 4B St. Dev.
\end{tabular}}} & & & & & 69.86 & & 66.12 & 33.88 & 36.7 \\
\hline & & & & & & 0.22 & & 0.49 & 0.49 & 0.49 \\
\hline
\end{tabular}

Recipe 4B St. Dev.

1) OC (Organic Carbon) calculated using the following equation: $O C=(1-F S) / 1.8$ (Haug 1993, Liao 1995), where OC and FS are based on the dry weight fri 
Phase 2 - Weekly Solids Analysis

November 5, 2002 Day 158

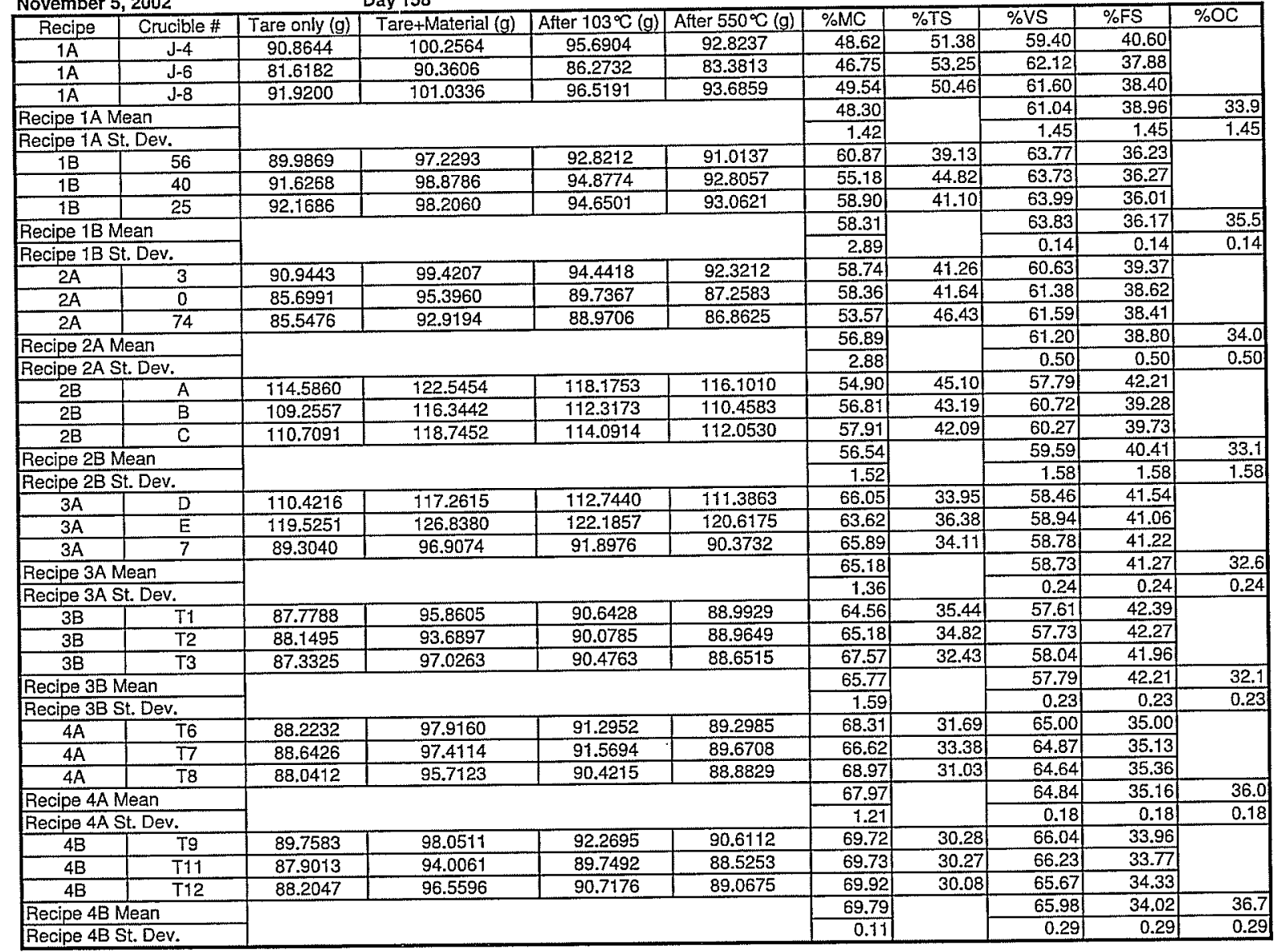

Recipe 4B St. Dev.

1) $\mathrm{OC}$ (Organic Carbon) calculated using the following equation: $\mathrm{OC}=(1-\mathrm{FS}) / 1.8$ (Haug 1993, Liao 1995), where OC and FS are based on the dry weight fr 
Phase 2 - Weekly Solids Analysis

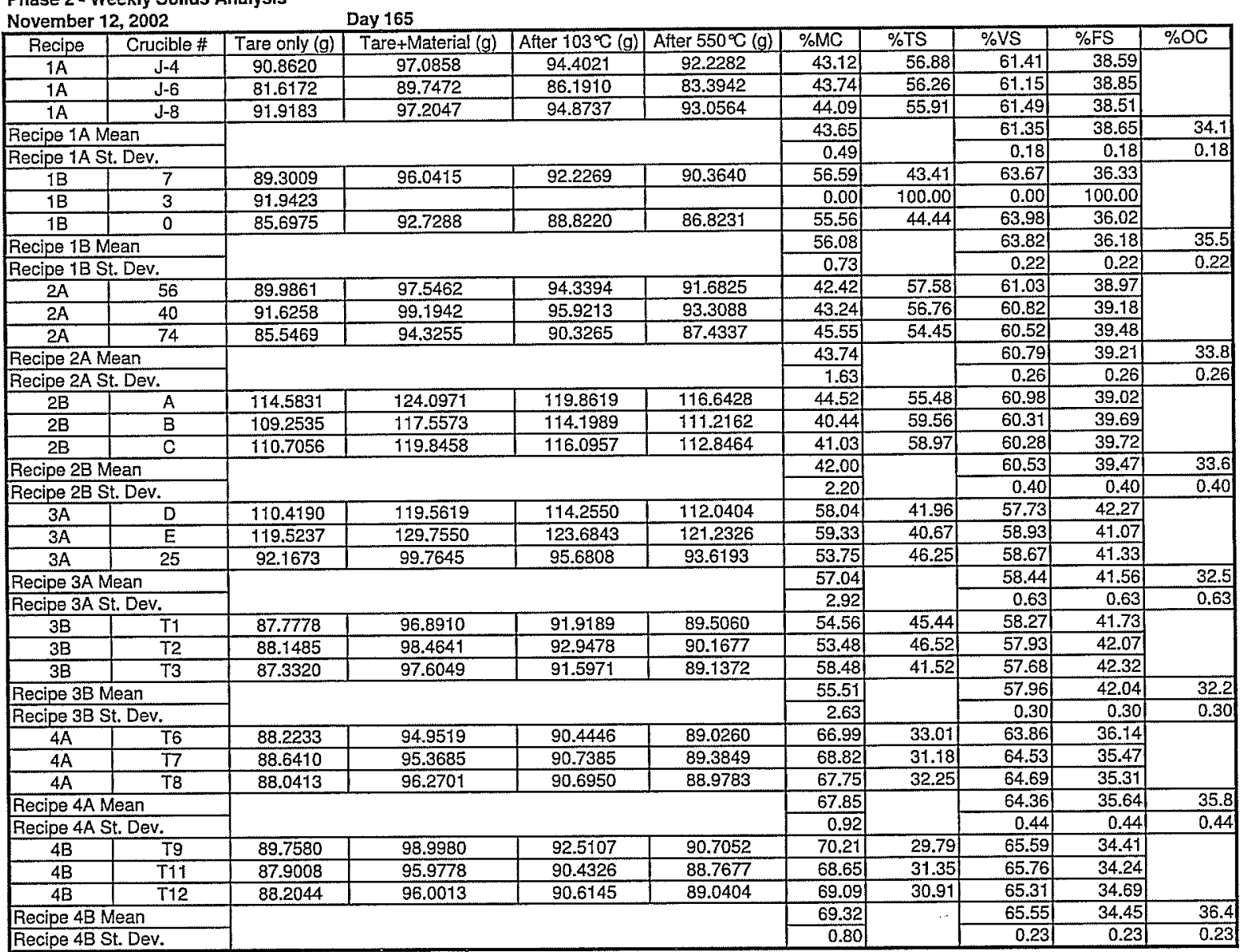

Recipe 4B St. Dev.

1) OC (Organic Carbon) calculated using the following equation: OC = (1-FS)/1.8 (Haug 1993, Liao 1995), where OC and FS are based on the dry weight fri 
Phase 2 - Weekly Solids Analysis

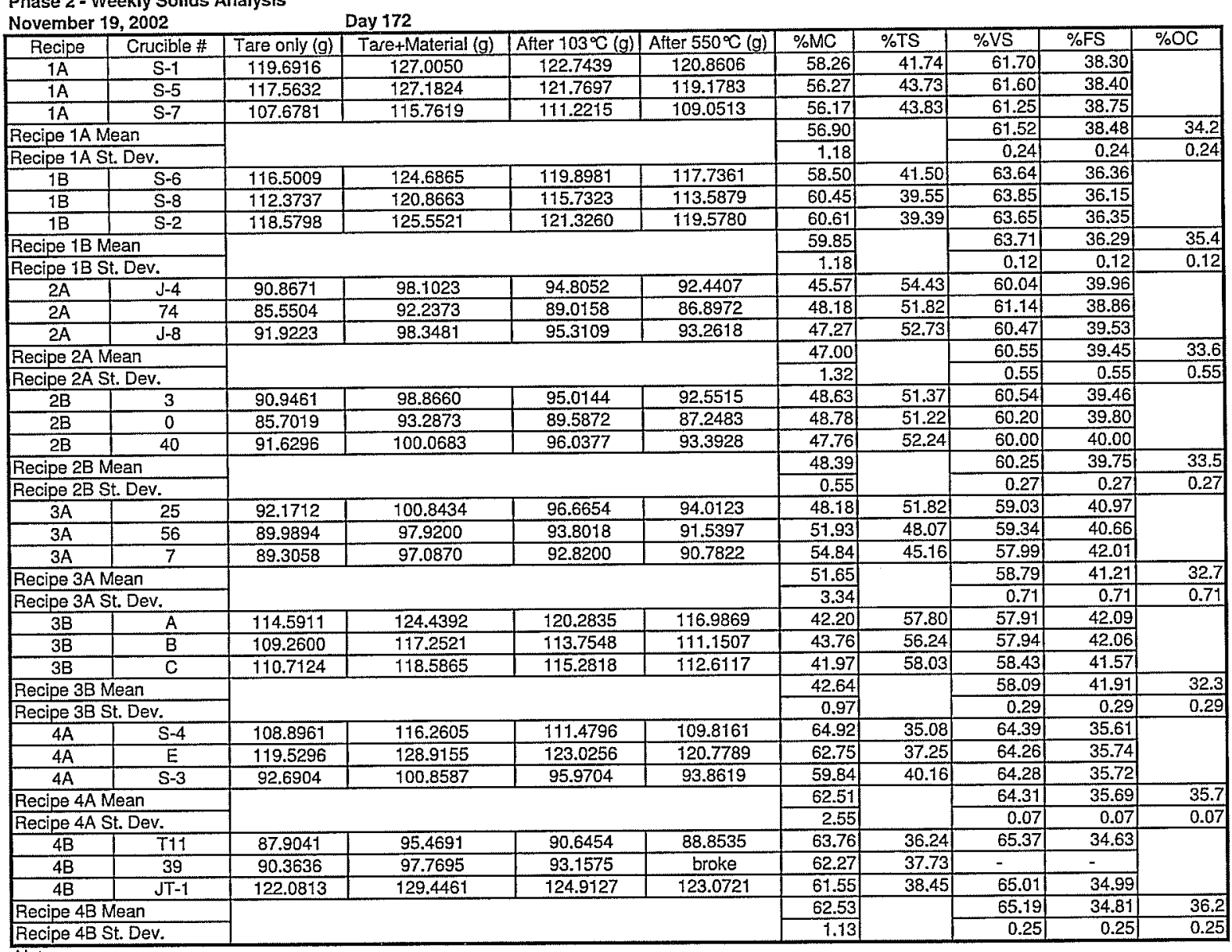

Notes:

1) OC (Organic Carbon) calculated using the following equation: OC = (1-FS)/1.8 (Haug 1993, Liao 1995), where OC and FS are based on the dry weight fri 
Phase 2 - Weekly Solids Analysis

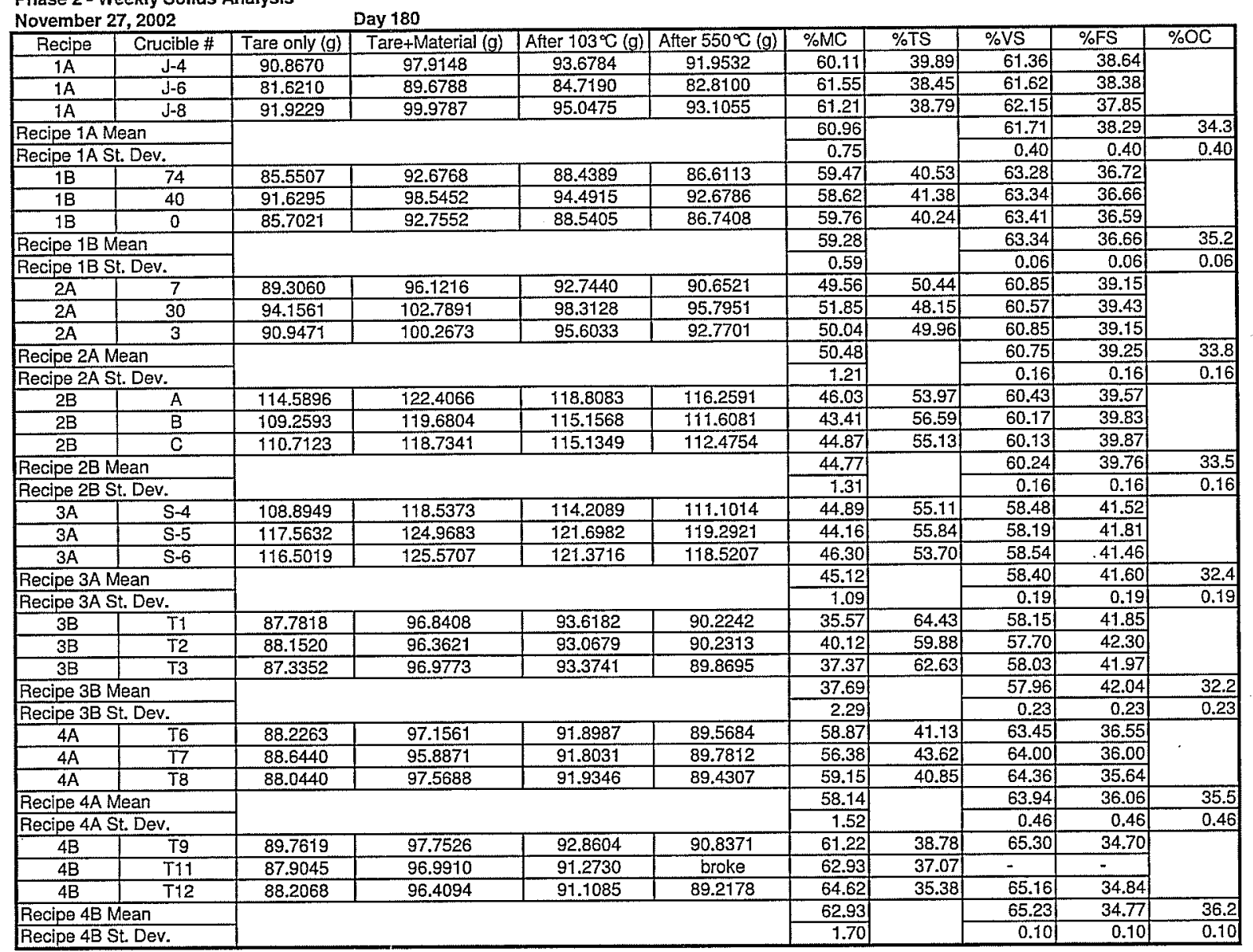

Notes:

1) OC (Organic Carbon) calculated using the following equation: OC = (1-FS)/1.8 (Haug 1993, Liao 1995), where OC and FS are based on the dry weight fri 
Phase 2- Summary of Weekly Volatile Solids Analysis

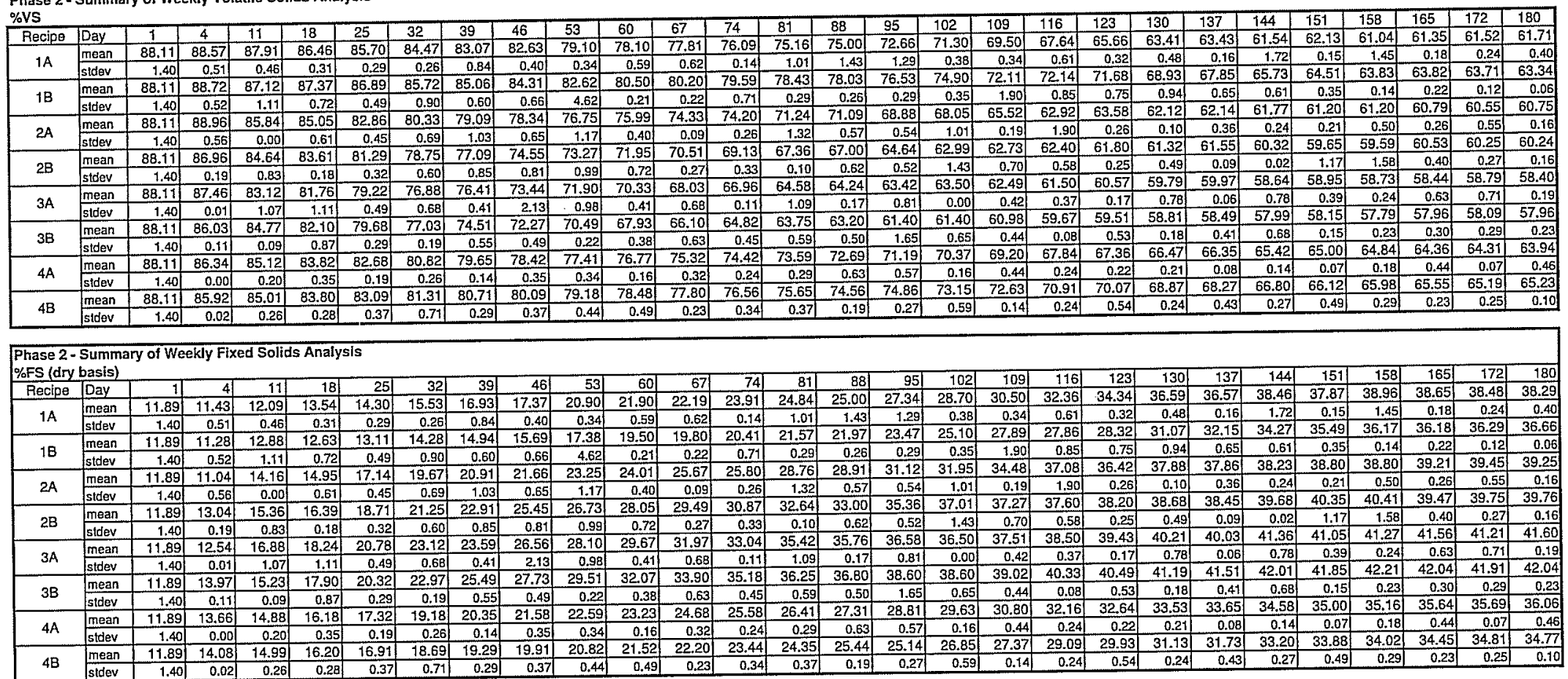


Phase 2 - Summary of Weekly Organic Carbon Analysis

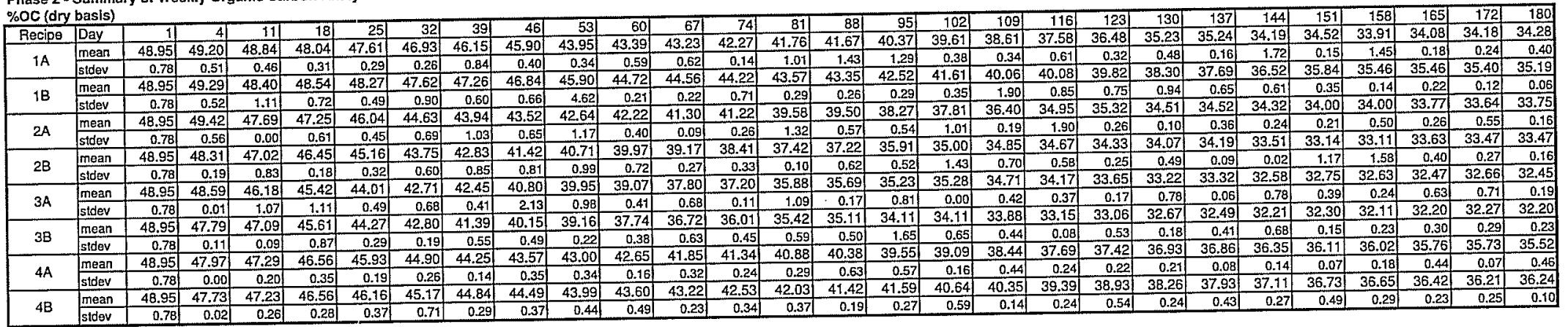

Notes: $O$ (Organic Carbon) calculated using the following equation: $O C=(1-F S) / 1.8$ (Haug 1993, Liao 1995), where $O C$ and FS are based on the dry weight fraction.

Phase 2 - Summary of Weekly Volatile Solids Analysis

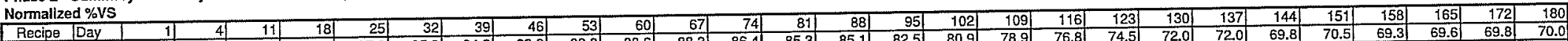

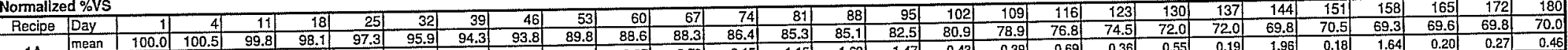

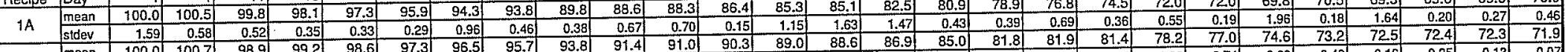

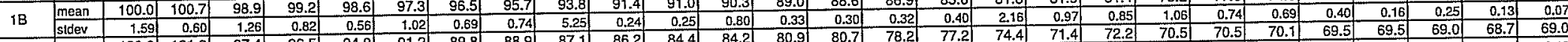

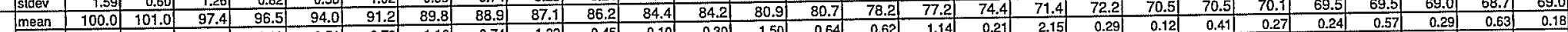

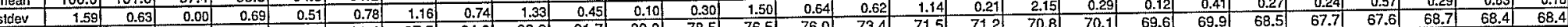

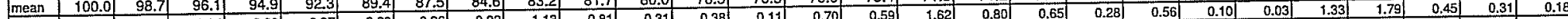

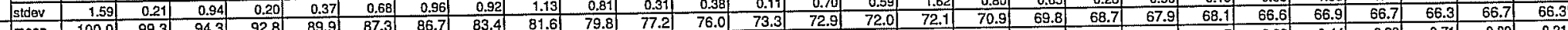

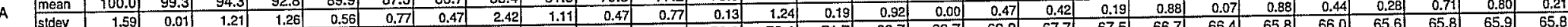

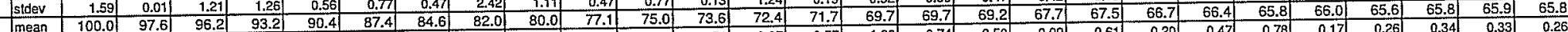

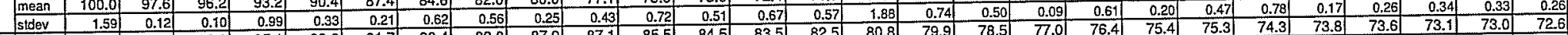

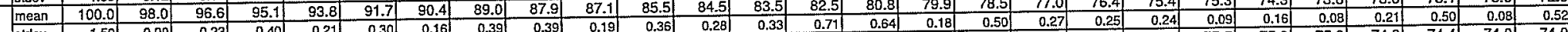

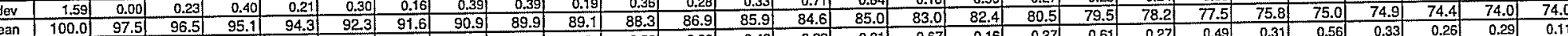


Phase 2 - Summary of Weekly Volatile Sollds Analysis

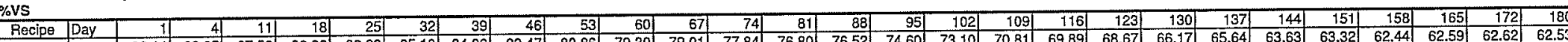

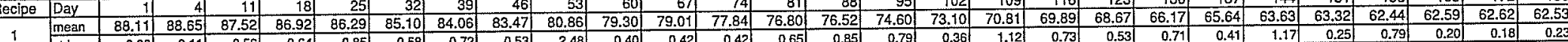

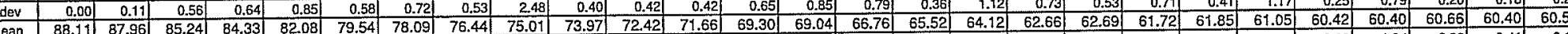

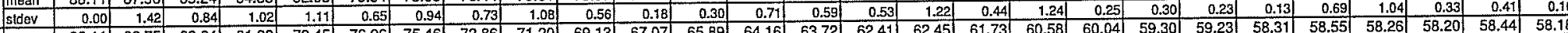

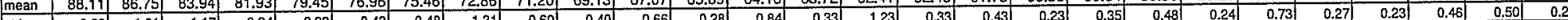

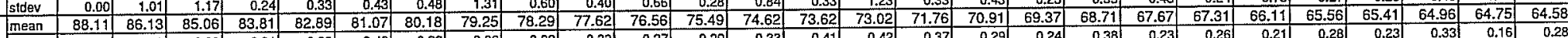

1) Shown as averages of duplicate recipes

Phase 2 - Summary of Weekly Volatile Sollds Analysis

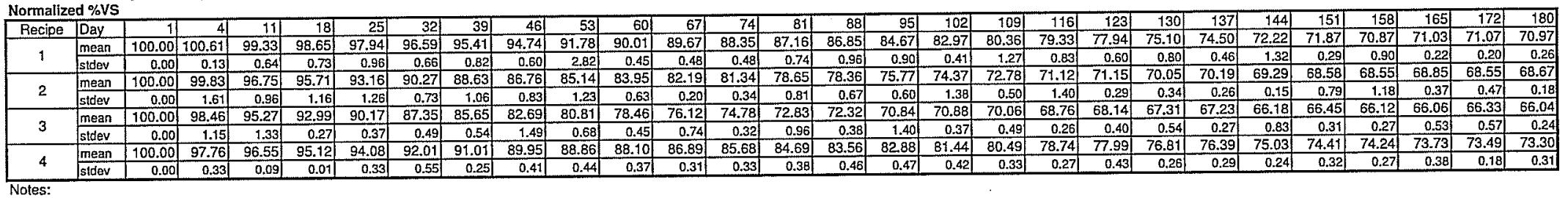

1) Shown as averages of duplicate recipes 
Phase 2 - Wet Welght of Tare \& Compost Material (kg)

\begin{tabular}{|l|l|l|l|l|l|l|l|l|l|l|l|l|l|l|l|l|l|l|l|l|l|l|l|l|l|l|l|l|l|l|l|l|l|l|}
\hline Recipe & Tare WL & 1 & 4 & 11 & 18 & 25 & 32 & 39 & 46 & 53 & 60 & 67 & 74 & 81 & 88 & 95 & 102 & 103 & 116 & 123 & 130 & 137 & 144 & 151 & 158 & 165 & 172 & 180 \\
\hline
\end{tabular}

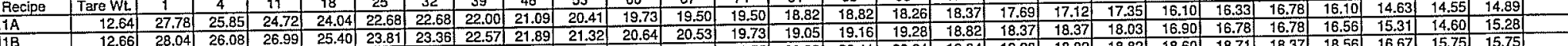

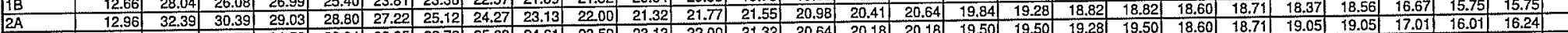

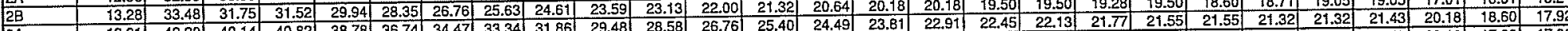

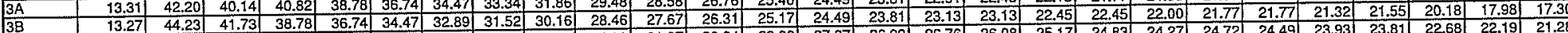

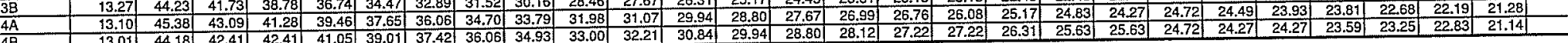
Notes: 1) All weights were recorded after moisture ad]ustment and sampling. 2) Tare + material weight was not recorded for Day 180 .

Phase 2 - Wet Welght of Compost Materlal (kg)

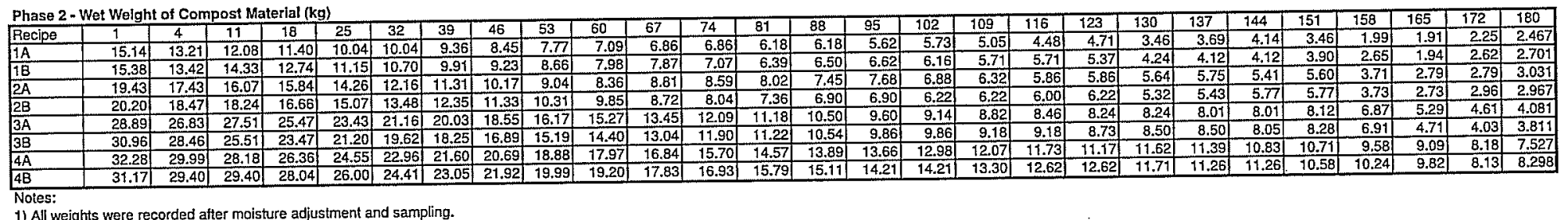
1) All weights were recorded after moisture adjustment and sampling.

Phase 2 - Wet Weight of Compost Material Normalized (\%)

\begin{tabular}{|l|l|l|l|l|l|l|l|l|l|l|l|l|l|l|l|l|l|l|l|l|l|l|l|l|l|l|l|l|l|l|}
\hline Recipe & 1 & 4 & 11 & 18 & 25 & 32 & 39 & 46 & 53 & 60 & 67 & 74 & 81 & 88 & 95 & 102 & 109 & 116 & 123 & 130 & 137 & 144 & 151 & 158 & 165 & 172 & 180 \\
\hline
\end{tabular}

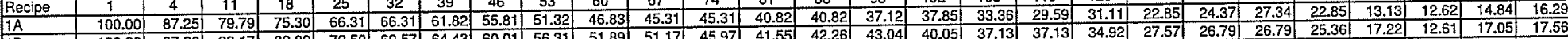

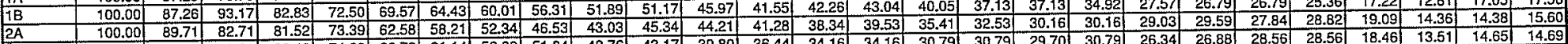

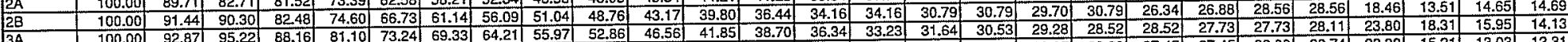

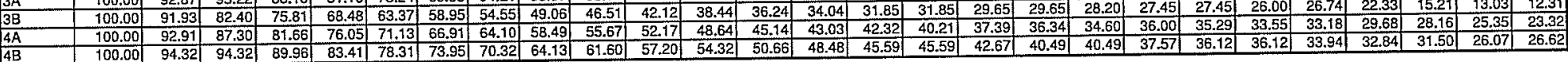




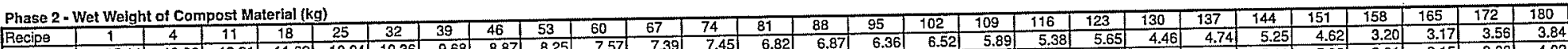

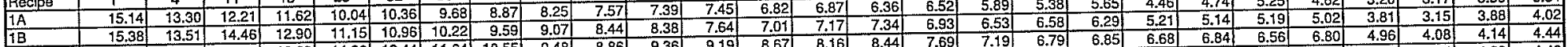

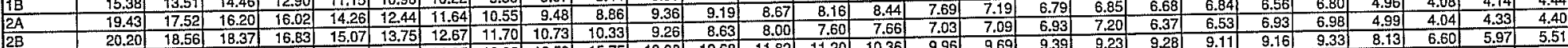

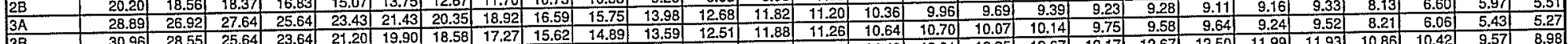

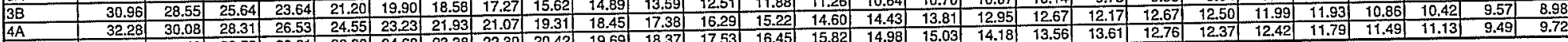

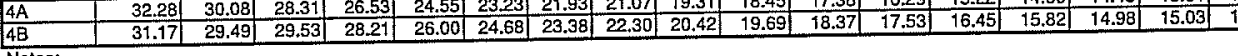

1) Includes correction for mass of compost sample removed from reactor weekly (i.e.removed sample welght is added to wet weight of compost material).

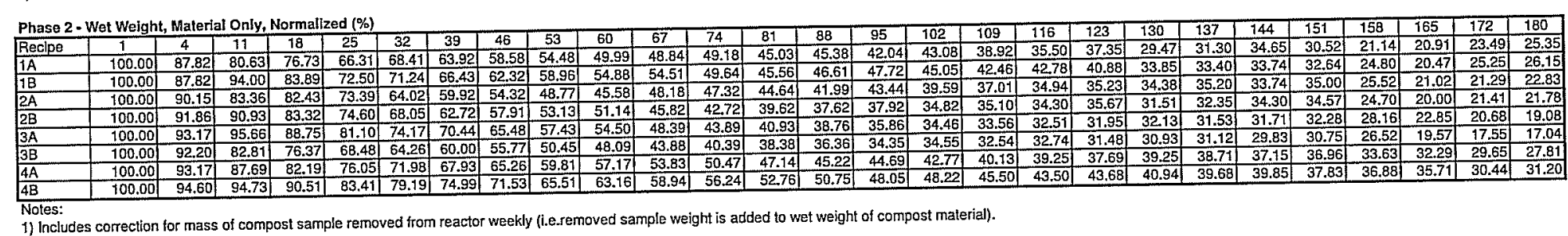

1) Includes correction for mass of compost sample removed from reactor weekly (1.e.removed sample weigh is addedlowetreght of co 


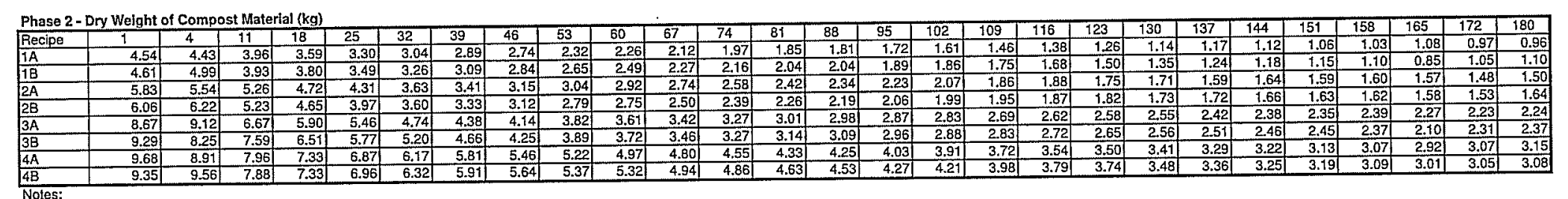
Noles:
1) Dry weights calculated from wet weights using \% Moisture Content - (wet weight - dry weight)/wet weight

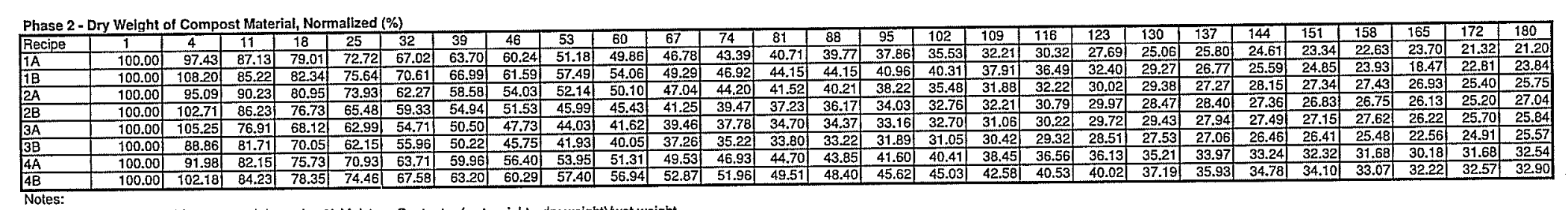

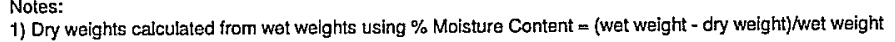




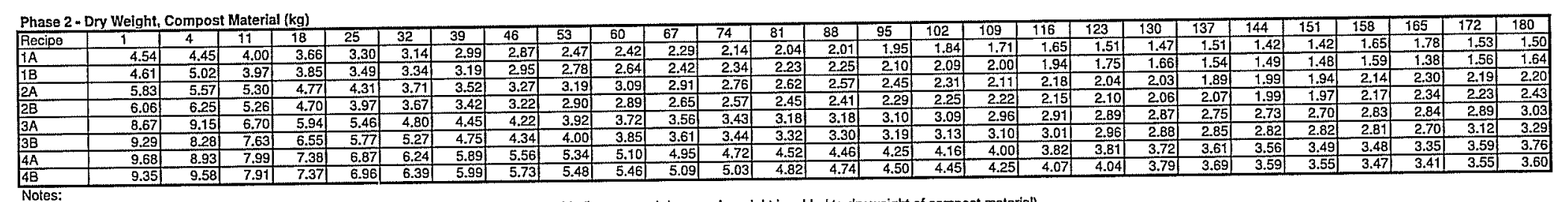
1) Includes correctlon for mass of compost sample (dry mass) removed from reactor weeky (..e.removed dry sample welght is added to dry weight of compost material). 2) Dry weights calculated from wet weights using \% Moisture Content $=$ (wet weight - dry weight)/wet weight

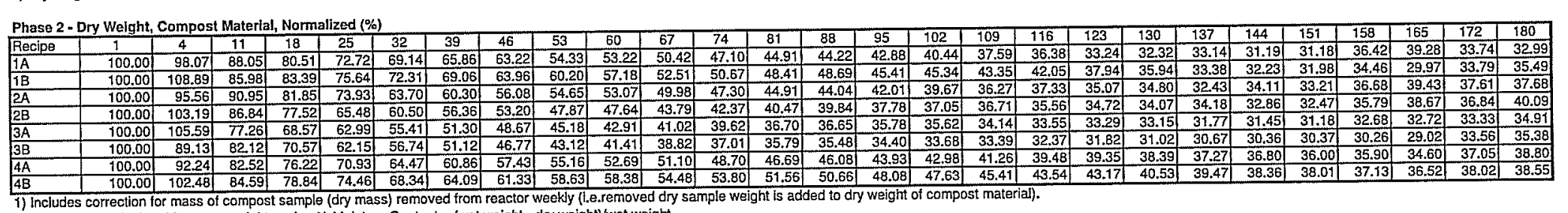

1) Includes correction tor mass of compost sample (dry mass) removed from reactor weekly (l.e.removed dry sample weight is added to dry weight of compost material).
2) Dry weights calculated from wet weights using \% Moisture Content $=$ (wet weight - dry weight)/wet weight 
Phase 2 - Moisture Content Measurements

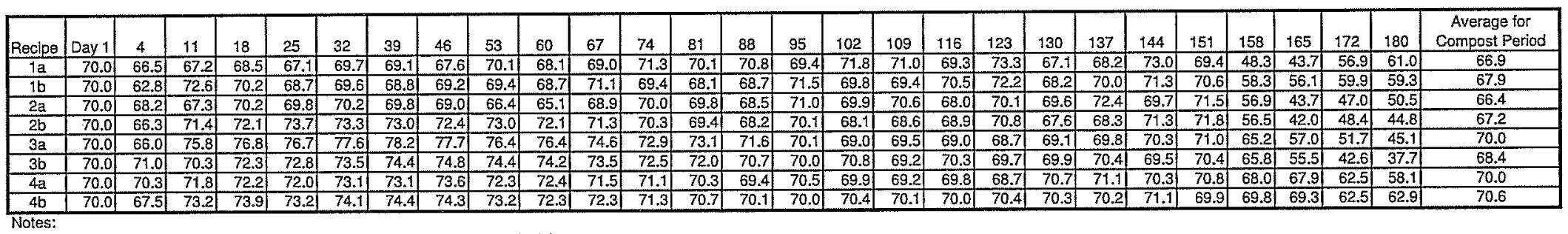

1) Values indicate moisture contents of compost after moisture addifion and mixing. 


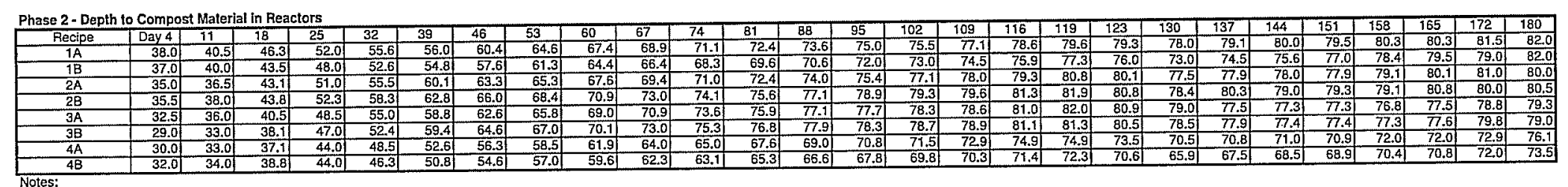

All measurements are in cm measured from top of composting reactor.

2) Depths were measured with applied weights in place and prior to mixing/sampling.

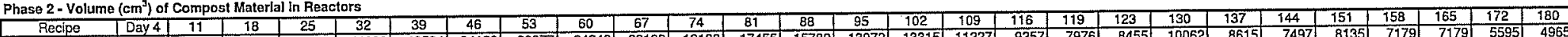

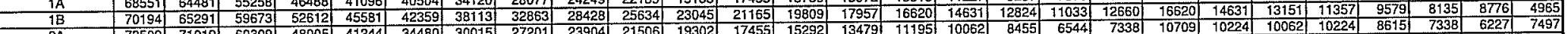

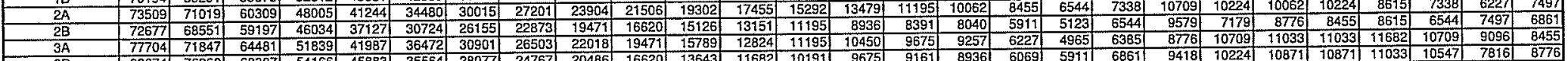

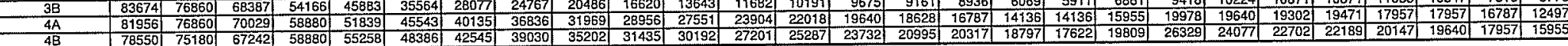
Notes:

1) All measurements are in $\mathrm{cm}^{3}$. 
Phase 2 - Normalized Volume $\left(\mathrm{cm}^{3}\right)$ of Compost Material in Reactors

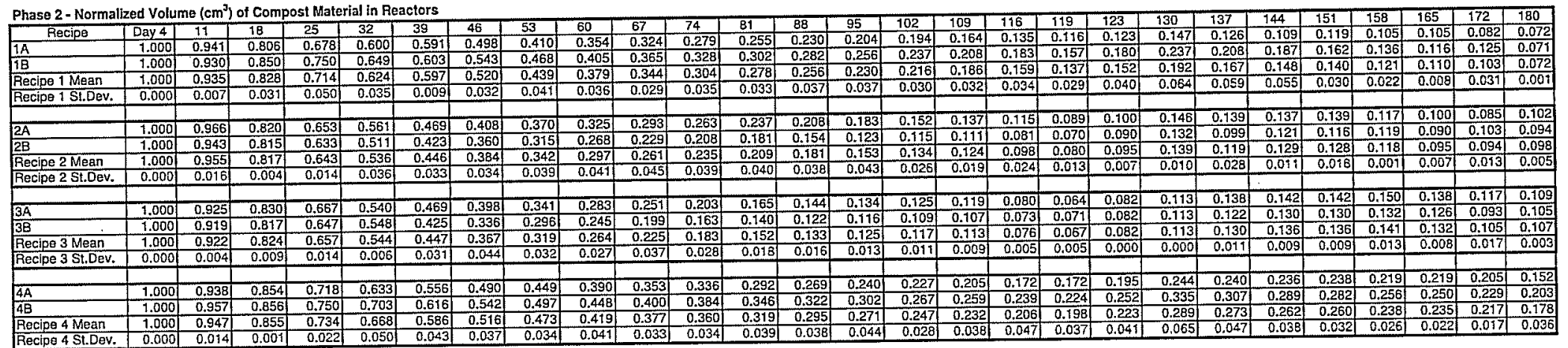


Phase 2 - Reactor and Sampling Masses (wet basis)

\begin{tabular}{|c|c|c|c|c|c|c|c|c|}
\hline \multirow[t]{2}{*}{ Day } & \multirow[t]{2}{*}{ Reactor } & $\begin{array}{c}\text { Tare } \\
\text { Weight }\end{array}$ & $\begin{array}{c}\text { Initial Mass } \\
\text { (material+tare) }\end{array}$ & $\begin{array}{c}\text { Initial Mass } \\
\text { (material only) }\end{array}$ & $\begin{array}{l}\text { Mass After Water } \\
\text { Addition and } \\
\text { Sampling } \\
\text { (material+tare) }\end{array}$ & \begin{tabular}{|} 
Mass After Water \\
Addition and \\
Sampling \\
(material only) \\
\end{tabular} & $\begin{array}{c}\text { Mass } \\
\text { Sampled }\end{array}$ & $\begin{array}{c}\text { Cumulative Mass } \\
\text { Sampled }\end{array}$ \\
\hline & & $(\mathrm{kg})$ & (b) & $(\mathrm{kg})$ & (b) & $(\mathrm{kg})$ & (g) & (g) \\
\hline \multirow[t]{8}{*}{ Day 4} & $1 \mathrm{~A}$ & 12.64 & 58.0 & 13.67 & 57.0 & 13.21 & 86.20 & 86.20 \\
\hline & $1 \mathrm{~B}$ & 12.66 & 58.5 & 13.88 & 57.5 & 13.42 & 86.20 & 86.20 \\
\hline & $2 A$ & 12.96 & 68.5 & 18.11 & 67.0 & 17.43 & 86.20 & 86.20 \\
\hline & $2 \mathrm{~B}$ & 13.28 & 71.0 & 18.93 & 70.0 & 18.47 & 86.20 & 86.20 \\
\hline & $3 A$ & 13.31 & 90.0 & 27.51 & 88.5 & 26.83 & 86.20 & 86.20 \\
\hline & $3 \mathrm{~B}$ & 13.27 & 94.5 & 29.59 & 92.0 & 28.46 & 86.20 & 86.20 \\
\hline & $4 \mathrm{~A}$ & 13.10 & 96.0 & 30.44 & 95.0 & 29.99 & 86.20 & 86.20 \\
\hline & $4 \mathrm{~B}$ & 13.01 & 97.5 & 31.22 & 93.5 & 29.40 & 86.20 & 86.20 \\
\hline \multirow[t]{8}{*}{ Day 11} & $1 \mathrm{~A}$ & 12.64 & 55.0 & 12.31 & 54.5 & 12.08 & 41.20 & 127.40 \\
\hline & $1 \mathrm{~B}$ & 12.66 & 60.5 & 14.78 & 59.5 & 14.33 & 41.20 & 127.40 \\
\hline & $2 \mathrm{~A}$ & 12.96 & 64.5 & 16.30 & 64.0 & 16.07 & 41.20 & 127.40 \\
\hline & $2 \mathrm{~B}$ & 13.28 & 70.0 & 18.47 & 69.5 & 18.24 & 41.20 & 127.40 \\
\hline & $3 \mathrm{~A}$ & 13.31 & 91.0 & 27.97 & 90.0 & 27.51 & 41.20 & 127.40 \\
\hline & 38 & 13.27 & 86.5 & 25.97 & 85.5 & 25.51 & 41.20 & 127.40 \\
\hline & $4 \mathrm{~A}$ & 13.10 & 92.5 & 28.86 & 91.0 & 28.18 & 41.20 & 127.40 \\
\hline & $4 B$ & 13.01 & 95.0 & 30.08 & 93.5 & 29.40 & 41.20 & 127.40 \\
\hline \multirow[t]{8}{*}{ Day 18} & $1 \mathrm{~A}$ & 12.64 & 54.0 & 11.85 & 53.0 & 11.40 & 36.2 & 163.60 \\
\hline & $1 \mathrm{~B}$ & 12.66 & 56.5 & 12.97 & 56.0 & 12.74 & 35.2 & 162.60 \\
\hline & $2 \mathrm{~A}$ & 12.96 & 64.0 & 16.07 & 63.5 & 15.84 & 48.2 & 175.60 \\
\hline & $2 B$ & 13.28 & 66.0 & 16.66 & 66.0 & 16.66 & 44.2 & 171.60 \\
\hline & $3 A$ & 13.31 & 86.0 & 25.70 & 85.5 & 25.47 & 41.2 & 168.60 \\
\hline & $3 B$ & 13.27 & 82.0 & 23.92 & 81.0 & 23.47 & 47.2 & 174.60 \\
\hline & $4 \mathrm{~A}$ & 13.10 & 88.0 & 26.82 & 87.0 & 26.36 & 43.2 & 170.60 \\
\hline & $4 \mathrm{~B}$ & 13.01 & 91.0 & 28.27 & 90.5 & 28.04 & 45.2 & 172.60 \\
\hline \multirow[t]{8}{*}{ Day 25} & $1 \mathrm{~A}$ & 12.64 & 50.5 & 10.27 & 50.0 & 10.04 & 53.2 & 216.80 \\
\hline & $1 B$ & 12.66 & 53.5 & 11.61 & 52.5 & 11.15 & 50.2 & 212.80 \\
\hline & $2 \mathrm{~A}$ & 12.96 & 60.5 & 14.48 & 60.0 & 14.26 & 53.2 & 228.80 \\
\hline & 28 & 13.28 & 63.0 & 15.30 & 62.5 & 15.07 & 53.2 & 224.80 \\
\hline & $3 A$ & 13.31 & 82.5 & 24.11 & 81.0 & 23.43 & 49.2 & 217.80 \\
\hline & $3 \mathrm{~B}$ & 13.27 & 77.0 & 21.66 & 76.0 & 21.20 & 50.2 & 224.80 \\
\hline & $4 \mathrm{~A}$ & 13.10 & 84.0 & 25.00 & 83.0 & 24.55 & 54.2 & 224.80 \\
\hline & $4 \mathrm{~B}$ & 13.01 & 86.5 & 26.23 & 86.0 & 26.00 & 50.2 & 222.80 \\
\hline \multirow[t]{8}{*}{ Day 32} & $1 \mathrm{~A}$ & 12.64 & 51.0 & 10.49 & 50.0 & 10.04 & 49.2 & 266.00 \\
\hline & $1 \mathrm{~B}$ & 12.66 & 52.0 & 10.93 & 51.5 & 10.70 & 44.2 & 257.00 \\
\hline & $2 A$ & 12.96 & 57.0 & 12.89 & 55.4 & 12.16 & 51.2 & 280.00 \\
\hline & $2 \mathrm{~B}$ & 13.28 & 59.8 & 13.82 & 59.0 & 13.48 & 42.2 & 267.00 \\
\hline & $3 A$ & 13.31 & 77.3 & 21.73 & 76.0 & 21.16 & 51.2 & 269.00 \\
\hline & $3 \mathrm{~B}$ & 13.27 & 74.0 & 20.30 & 72.5 & 19.62 & 50.2 & 275.00 \\
\hline & $4 \mathrm{~A}$ & 13.10 & 80.5 & 23.41 & 79.5 & 22.96 & 49.2 & 274.00 \\
\hline & $4 B$ & 13.01 & 83.5 & 24.86 & 82.5 & 24.41 & 50.2 & 273.00 \\
\hline \multirow[t]{8}{*}{ Day 39} & $1 \mathrm{~A}$ & 12.64 & 48.5 & 9.36 & 48.5 & 9.36 & 51.2 & 317.20 \\
\hline & $1 B$ & 12.66 & 50.3 & 10.13 & 49.8 & 9.91 & 49.2 & 306.20 \\
\hline & $2 A$ & 12.96 & 54.0 & 11.53 & 53.5 & 11.31 & 53.2 & 333.20 \\
\hline & $2 \mathrm{~B}$ & 13.28 & 56.5 & 12.35 & 56.5 & 12.35 & 52.2 & 319.20 \\
\hline & $3 A$ & 13.31 & 74.5 & 20.48 & 73.5 & 20.03 & 51.2 & 320.20 \\
\hline & 3B & 13.27 & 70.0 & 18.48 & 69.5 & 18.25 & 51.2 & 326.20 \\
\hline & $4 \mathrm{~A}$ & 13.10 & 78.0 & 22.28 & 76.5 & 21.60 & 52.2 & 326.20 \\
\hline & $4 \mathrm{~B}$ & 13.01 & 80.5 & 23.50 & 79.5 & 23.05 & 52.2 & 325.20 \\
\hline
\end{tabular}


Phase 2 - Reactor and Sampling Masses (wet basis)

\begin{tabular}{|c|c|c|c|c|c|c|c|c|}
\hline \multirow[t]{2}{*}{ Day } & \multirow[t]{2}{*}{ Reactor } & $\begin{array}{c}\text { Tare } \\
\text { Weight }\end{array}$ & $\begin{array}{c}\text { Initial Mass } \\
\text { (material+tare) }\end{array}$ & $\begin{array}{c}\text { Initial Mass } \\
\text { (material only) }\end{array}$ & $\begin{array}{c}\text { Mass Afler Water } \\
\text { Addition and } \\
\text { Sampling } \\
\text { (material+tare) } \\
\end{array}$ & $\begin{array}{c}\text { Mass After Water } \\
\text { Additton and } \\
\text { Sampling } \\
\text { (material only) } \\
\end{array}$ & $\begin{array}{c}\text { Mass } \\
\text { Sampled }\end{array}$ & $\begin{array}{c}\begin{array}{c}\text { Cumulative Mass } \\
\text { Sampled }\end{array} \\
\end{array}$ \\
\hline & & $(\mathrm{kg})$ & (b) & $(\mathrm{kg})$ & $(\mathrm{b})$ & $(\mathrm{kg})$ & (g) & \begin{tabular}{|c|} 
(g) \\
\end{tabular} \\
\hline \multirow[t]{8}{*}{ Day 46} & $1 \mathrm{~A}$ & 12.64 & 47.0 & 8.68 & 46.5 & 8.45 & 48.2 & 365.40 \\
\hline & $1 \mathrm{~B}$ & 12.66 & 48.5 & 9.34 & 48.3 & 9.23 & 49.2 & 355.40 \\
\hline & $2 \mathrm{~A}$ & 12.96 & 51.5 & 10.40 & 51.0 & 10.17 & 51.2 & 384.40 \\
\hline & $2 B$ & 13.28 & 54.8 & 11.55 & 54.3 & 11.33 & 49.2 & 368.40 \\
\hline & $3 A$ & 13.31 & 71.5 & 19.12 & 70.3 & 18.55 & 47.2 & 367.40 \\
\hline & $3 \mathrm{~B}$ & 13.27 & 68.0 & 17.57 & 66.5 & 16.89 & 50.2 & 376.40 \\
\hline & $4 \mathrm{~A}$ & 13.10 & 75.3 & 21.03 & 74.5 & 20.69 & 49.2 & 375.40 \\
\hline & 4B & 13.01 & 78.0 & 22.37 & 77.0 & 21.92 & 51.2 & 376,40 \\
\hline \multirow[t]{8}{*}{ Day 53} & $1 \mathrm{~A}$ & 12.64 & 45.0 & 7.77 & 45.0 & 7.77 & 53.2 & 418.60 \\
\hline & $1 B$ & 12.66 & 46.8 & 8.55 & 47.0 & 8.66 & 52.2 & 407.60 \\
\hline & $2 \mathrm{~A}$ & 12.96 & 49.5 & 9.49 & 48.5 & 9.04 & 51.2 & 435.60 \\
\hline & 28 & 13.28 & 53.3 & 10.87 & 52.0 & 10.31 & 54.2 & 422,60 \\
\hline & $3 \mathrm{~A}$ & 13.31 & 68.5 & 17.76 & 65.0 & 16.17 & 54.2 & 421.60 \\
\hline & $3 \mathrm{~B}$ & 13.27 & 65.0 & 16.21 & 62.8 & 15.19 & 53.2 & 429.60 \\
\hline & $4 \mathrm{~A}$ & 13.10 & 72.8 & 19.90 & 70.5 & 18.88 & 51.2 & 426.60 \\
\hline & $4 \mathrm{~B}$ & 13.01 & 75.3 & 21.12 & 72.8 & 19.99 & 54.2 & 430.60 \\
\hline \multirow[t]{8}{*}{ Day 60} & $1 \mathrm{~A}$ & 12.64 & 44.0 & 7.32 & 43.5 & 7.09 & 59.2 & 477.80 \\
\hline & $1 \mathrm{~B}$ & 12.66 & 46.0 & 8.21 & 45.5 & 7.98 & 52.2 & 459.80 \\
\hline & $2 A$ & 12.96 & 47.3 & 8.47 & 47.0 & 8.36 & 61.2 & 496.80 \\
\hline & $2 B$ & 13.28 & 51.3 & 9.97 & 51.0 & 9.85 & 57.2 & 479.80 \\
\hline & $3 A$ & 13.31 & 64.0 & 15.72 & 63.0 & 15.27 & 54.2 & 475.80 \\
\hline & $3 B$ & 13.27 & 61.8 & 14.74 & 61.0 & 14.40 & 59.2 & 488.80 \\
\hline & $4 \mathrm{~A}$ & 13.10 & 69.5 & 18.42 & 68.5 & 17.97 & 58.2 & 484.80 \\
\hline & $4 B$ & 13.01 & 71.5 & 19.42 & 71.0 & 19.20 & 57.2 & 487.80 \\
\hline \multirow[t]{8}{*}{ Day 67} & $1 \mathrm{~A}$ & 12.64 & 42.5 & 6.64 & 43.0 & 6.86 & 56.2 & 534.00 \\
\hline & $1 \mathrm{~B}$ & 12.66 & 44.5 & 7.52 & 45.3 & 7.87 & 53.2 & 513.00 \\
\hline & $2 \mathrm{~A}$ & 12.96 & 46.0 & 7.91 & 48.0 & 8.81 & 54.2 & 551.00 \\
\hline & $2 \mathrm{~B}$ & 13.28 & 49.0 & 8.95 & 48.5 & 8.72 & 55.2 & 535.00 \\
\hline & $3 A$ & 13.31 & 61.3 & 14.47 & 59.0 & 13.45 & 55.2 & 531.00 \\
\hline & 38 & 13.27 & 59.3 & 13.61 & 58.0 & +3.04 & 57.2 & 546.00 \\
\hline & $4 \mathrm{~A}$ & 13.10 & 67.0 & 17.29 & 66.0 & 16.84 & 52.2 & 537.00 \\
\hline & $4 \mathrm{~B}$ & 13.01 & 69.5 & 18.51 & 68.0 & 17.83 & 53.2 & 541.00 \\
\hline \multirow[t]{8}{*}{ Day 74} & $1 \mathrm{~A}$ & 12.64 & 42.0 & 6.41 & 43.0 & 6.86 & 52.2 & 586.20 \\
\hline & 18 & 12.66 & 44.0 & 7.30 & 43.5 & 7.07 & 52.2 & 565.20 \\
\hline & $2 \mathrm{~A}$ & 12.96 & 47.0 & 8.36 & 47.5 & 8.59 & 53.2 & 604.20 \\
\hline & $2 \mathrm{~B}$ & 13.28 & 47.5 & 8.27 & 47.0 & 8.04 & 55.2 & 590.20 \\
\hline & $3 \mathrm{~A}$ & 13.31 & 57.8 & 12.88 & 56.0 & 12.09 & 59.2 & 590.20 \\
\hline & $3 \mathrm{~B}$ & 13.27 & 57.0 & 12.58 & 55.5 & 11.90 & 59.2 & 605.20 \\
\hline & $4 A$ & 13.10 & 65.0 & 16.38 & 63.5 & 15.70 & 55.2 & 592.20 \\
\hline & $4 B$ & 13.01 & 67.0 & 17.38 & 66.0 & 16.93 & 59.2 & 600.20 \\
\hline \multirow[t]{8}{*}{ Day 81 } & $1 \mathrm{~A}$ & 12.64 & 42.0 & 6.41 & 41.5 & 6.18 & 51.2 & 637.40 \\
\hline & IB & 12.66 & 42.5 & 6.62 & 42.0 & 6.39 & 51.2 & 616.40 \\
\hline & $2 A$ & 12.96 & 46.8 & 8.25 & 46.3 & 8.02 & 49.2 & 653.40 \\
\hline & $2 B$ & 13.28 & 46.0 & 7.59 & 45.5 & 7.36 & 52.2 & 642.40 \\
\hline & $3 A$ & 13.31 & 55.0 & 11.64 & 54.0 & 11.18 & 54.2 & 644.40 \\
\hline & $3 \mathrm{~B}$ & 13.27 & 54.5 & 11.45 & 54.0 & 11.22 & 56.2 & 661.40 \\
\hline & $4 A$ & 13.10 & 62.0 & 15.02 & 61.0 & 14.57 & 56.2 & 648.40 \\
\hline & $4 \mathrm{~B}$ & 13.01 & 64.8 & 16.36 & 63.5 & 15.79 & 55.2 & 655.40 \\
\hline \multirow[t]{8}{*}{ Day 88} & $1 \mathrm{~A}$ & 12.64 & 40.5 & 5.73 & 41.5 & 6.18 & 53.2 & 690.60 \\
\hline & $1 \mathrm{~B}$ & 12.66 & 41.5 & 6.16 & 42.3 & 6.50 & 52.2 & 668.60 \\
\hline & $2 A$ & 12.96 & 45.0 & 7.45 & 45.0 & 7.45 & 56.2 & 709.60 \\
\hline & $2 B$ & 13.28 & 44.5 & 6.90 & 44.5 & 6.90 & 56.2 & 698.60 \\
\hline & $3 A$ & 13.31 & 52.5 & 10.50 & 52.5 & 10.50 & 53.2 & 697.60 \\
\hline & $3 \mathrm{~B}$ & 13.27 & 53.0 & 10.77 & 52.5 & 10.54 & 57.2 & 718.60 \\
\hline & $4 \mathrm{~A}$ & 13.10 & 60.0 & 14.12 & 59.5 & 13.89 & 58.2 & 706.60 \\
\hline & $4 \mathrm{~B}$ & 13.01 & 62.5 & 15.34 & 62.0 & 15.11 & 53.2 & 708.60 \\
\hline
\end{tabular}


Phase 2 - Reactor and Sampling Masses (wet basis)

\begin{tabular}{|c|c|c|c|c|c|c|c|c|}
\hline \multirow[t]{2}{*}{ Day } & \multirow[t]{2}{*}{ Reactor } & $\begin{array}{c}\text { Tare } \\
\text { Weight }\end{array}$ & $\begin{array}{c}\text { Initial Mass } \\
\text { (material+tare) }\end{array}$ & $\begin{array}{c}\text { Initial Mass } \\
\text { (material only) }\end{array}$ & $\begin{array}{c}\text { Mass After Water } \\
\text { Addition and } \\
\text { Sampling } \\
\text { (malerial+tare) } \\
\end{array}$ & $\begin{array}{c}\text { Mass After Water } \\
\text { Addition and } \\
\text { Sampling } \\
\text { (material only) } \\
\end{array}$ & $\begin{array}{c}\text { Mass } \\
\text { Sampled } \\
\end{array}$ & $\begin{array}{c}\text { Cumulative Mass } \\
\text { Sampled }\end{array}$ \\
\hline & & $(\mathrm{kg})$ & (b) & $(\mathrm{kg})$ & $(\mathrm{lb})$ & \begin{tabular}{|c|}
$(\mathrm{kg})$ \\
\end{tabular} & (g) & (g) \\
\hline \multirow[t]{8}{*}{ Day 95} & $1 \mathrm{~A}$ & 12.64 & 40.5 & 5.73 & 40.3 & 5.62 & 54.2 & 744.80 \\
\hline & $1 \mathrm{~B}$ & 12.66 & 41.0 & 5.94 & 42.5 & 6.62 & 50.2 & 718.80 \\
\hline & $2 A$ & 12.96 & 44.0 & 7.00 & 45.5 & 7.68 & 51.2 & 760.80 \\
\hline & $2 B$ & 13.28 & 43.5 & 6.45 & 44.5 & 6.90 & 61.2 & 759.80 \\
\hline & $3 \mathrm{~A}$ & 13.31 & 51.0 & 9.82 & 50.5 & 9.60 & 61.2 & 758.80 \\
\hline & $3 \mathrm{~B}$ & 13.27 & 51.5 & 10.09 & 51.0 & 9.86 & 57.2 & 775.80 \\
\hline & $4 \mathrm{~A}$ & 13.10 & 58.0 & 13.21 & 59.0 & 13.66 & 60.2 & 766.80 \\
\hline & $4 \mathrm{~B}$ & 13.01 & 60.5 & 14.43 & 60.0 & 14.21 & 59.2 & 767.80 \\
\hline \multirow[t]{8}{*}{ Day 102} & $1 \mathrm{~A}$ & 12.64 & 39.0 & 5.05 & 40.5 & 5.73 & 48.2 & 793.00 \\
\hline & $1 \mathrm{~B}$ & 12.66 & 42.0 & 6.39 & 41.5 & 6.16 & 50.2 & 769.00 \\
\hline & $2 \mathrm{~A}$ & 12.96 & 44.0 & 7.00 & 43.8 & 6.88 & 52.2 & 813.00 \\
\hline & $2 \mathrm{~B}$ & 13.28 & 43.5 & 6.45 & 43.0 & 6.22 & 54.2 & 814.00 \\
\hline & $3 \mathrm{~A}$ & $13.3\}$ & 49.5 & 9.14 & 49.5 & 9.14 & 57.2 & 816.00 \\
\hline & 38 & 13.27 & 50.5 & 9.64 & 51.0 & 9.86 & 60.2 & 836.00 \\
\hline & $4 \mathrm{~A}$ & 13.10 & 57.5 & 12.98 & 57.5 & 12.98 & 58.2 & 825.00 \\
\hline & $4 \mathrm{~B}$ & 13.01 & 59.0 & 13.75 & 60.0 & 14.21 & 53.2 & 821.00 \\
\hline \multirow[t]{8}{*}{ Day 109} & $1 \mathrm{~A}$ & 12.64 & 39.5 & 5.28 & 39.0 & 5.05 & 50.2 & 843.20 \\
\hline & 18 & 12.66 & 39.8 & 5.37 & 40.5 & 5.71 & 51.2 & 820.20 \\
\hline & $2 \mathrm{~A}$ & 12.96 & 42.5 & 6.32 & 42.5 & 6.32 & 57.2 & 870.20 \\
\hline & $2 B$ & 13.28 & 42.0 & 5.77 & 43.0 & 6.22 & 56.2 & 870.20 \\
\hline & $3 A$ & 13.31 & 48.5 & 8.69 & 48.8 & 8.82 & 58.2 & 874.20 \\
\hline & 38 & 13.27 & 49.0 & 8.96 & 49.5 & 9.18 & 58.2 & 894.20 \\
\hline & $4 \mathrm{~A}$ & 13.10 & 56.0 & 12.30 & 55.5 & 12.07 & 58.2 & 883.20 \\
\hline & $4 \mathrm{~B}$ & 13.01 & 58.8 & 13.64 & 58.0 & 13.30 & 61.2 & 882.20 \\
\hline \multirow[t]{8}{*}{ Day 116} & $1 \mathrm{~A}$ & 12.64 & 38.0 & 4.60 & 37.8 & 4.48 & 52.2 & 895.40 \\
\hline & $1 \mathrm{~B}$ & 12.66 & 39.3 & 5.14 & 40.5 & 5.71 & 49.2 & 869.40 \\
\hline & $2 A$ & 12.96 & 42.0 & 6.09 & 41.5 & 5.86 & 58.2 & 928.40 \\
\hline & $2 \mathrm{~B}$ & 13.28 & 41.0 & 5.32 & 42.5 & 6.00 & 58.2 & 928.40 \\
\hline & $3 \mathrm{~A}$ & 13.31 & 47.0 & 8.01 & 48.0 & 8.46 & 57.2 & 931.40 \\
\hline & 3B & 13.27 & 48.5 & 8.73 & 49.5 & 9.18 & 62.2 & 956.40 \\
\hline & $4 \mathrm{~A}$ & 13.10 & 54.0 & 11.39 & 54.8 & 11.73 & 55.2 & 938.40 \\
\hline & $4 B$ & 13.01 & 56.5 & 12.62 & 56.5 & 12.62 & 55.2 & 937.40 \\
\hline \multirow[t]{8}{*}{ Day 123} & $1 \mathrm{~A}$ & 12.64 & 36.5 & 3.92 & 38.3 & 4.71 & 49.2 & 944.60 \\
\hline & $1 \mathrm{~B}$ & 12.66 & 39.0 & 5.03 & 39.8 & 5.37 & 48.2 & 917.60 \\
\hline & $2 \mathrm{~A}$ & 12.96 & 39.5 & 4.96 & 41.5 & 5.86 & 57.2 & 985.60 \\
\hline & 28 & 13.28 & 41.3 & 5.43 & 43.0 & 6.22 & 56.2 & 984.60 \\
\hline & $3 \mathrm{~A}$ & 13.31 & 46.0 & 7.56 & 47.5 & 8.24 & 59.2 & 990.60 \\
\hline & $3 \mathrm{~B}$ & 13.27 & 48.0 & 8.50 & 48.5 & 8.73 & 59.2 & 1015.60 \\
\hline & $4 A$ & 13.10 & 52.8 & 10.83 & 53.5 & 11.17 & 59.2 & 997.60 \\
\hline & $4 \mathrm{~B}$ & 13.01 & 55.5 & 12.16 & 56.5 & 12.62 & 57.2 & 994.60 \\
\hline \multirow[t]{8}{*}{ Day 130} & $1 \mathrm{~A}$ & 12.64 & 36.0 & 3.69 & 35.5 & 3.46 & 57.2 & 1001.80 \\
\hline & $1 B$ & 12.66 & 37.5 & 4.35 & 37.3 & 4.24 & 49.2 & 966.80 \\
\hline & $2 \mathrm{~A}$ & 12.96 & 40.0 & 5.18 & 41.0 & 5.64 & 55.2 & 1040.80 \\
\hline & $2 \mathrm{~B}$ & 13.28 & 41.5 & 5.54 & 41.0 & 5.32 & 61.2 & 1045.80 \\
\hline & $3 \mathrm{~A}$ & 13.31 & 46.0 & 7.56 & 47.5 & 8.24 & 53.2 & 1043.80 \\
\hline & $3 \mathrm{~B}$ & 13.27 & 47.5 & 8.28 & 48.0 & 8.50 & 61.2 & 1076.80 \\
\hline & $4 \mathrm{~A}$ & 13.10 & 52.8 & 10.83 & 54.5 & 11.62 & 53.2 & 1050.80 \\
\hline & 4B & 13.01 & 55.0 & 11.94 & 54.5 & 11.71 & 56.2 & 1050.80 \\
\hline \multirow[t]{8}{*}{ Day 137} & $1 \mathrm{~A}$ & 12.64 & 34.0 & 2.78 & 36.0 & 3.69 & 47.2 & 1049.00 \\
\hline & B & 12.66 & 35.5 & 3.44 & 37.0 & 4.12 & 50.2 & 1017.00 \\
\hline & $2 \mathrm{~A}$ & 12.96 & 40.0 & 5.18 & 41.3 & 5.75 & 48.2 & 1089.00 \\
\hline & $2 \mathrm{~B}$ & 13.28 & 39.5 & 4.64 & 41.3 & 5.43 & 58.2 & 1104.00 \\
\hline & $3 A$ & 13.31 & 46.0 & 7.56 & 47.0 & 8.01 & 54.2 & 1098.00 \\
\hline & $3 \mathrm{~B}$ & 13.27 & 47.0 & 8.05 & 48.0 & 8.50 & 58.2 & 1135.00 \\
\hline & $4 \mathrm{~A}$ & 13.10 & 53.0 & 10.94 & 54.0 & 11.39 & 56.2 & 1107.00 \\
\hline & $4 \mathrm{~B}$ & 13.01 & 52.5 & 10.80 & 53.5 & 11.26 & 57.2 & 1108.00 \\
\hline
\end{tabular}


Phase 2 - Reactor and Sampling Masses (wet basis)

\begin{tabular}{|c|c|c|c|c|c|c|c|c|}
\hline \multirow[t]{2}{*}{ Day } & \multirow[t]{2}{*}{ Reactor } & $\begin{array}{c}\text { Tare } \\
\text { Weight }\end{array}$ & $\begin{array}{c}\text { Iniltial Mass } \\
\text { (material+tare) }\end{array}$ & $\begin{array}{c}\text { Initial Mass } \\
\text { (material only) }\end{array}$ & $\begin{array}{l}\text { Mass After Water } \\
\text { Addition and } \\
\text { Sampling } \\
\text { (material+lare) } \\
\end{array}$ & \begin{tabular}{|c|} 
Mass After Water \\
Addition and \\
Sampling \\
(material only) \\
\end{tabular} & $\begin{array}{c}\text { Mass } \\
\text { Sampled }\end{array}$ & $\begin{array}{c}\text { Cumulative Mass } \\
\text { Sampled } \\
\end{array}$ \\
\hline & & $(\mathrm{kg})$ & (b) & $(\mathrm{kg})$ & (b) & $(\mathrm{kg})$ & (g) & (g) \\
\hline \multirow[t]{8}{*}{ Day 144} & $1 \mathrm{~A}$ & 12.64 & 34.8 & 3.12 & 37.0 & 4.14 & 57.2 & 1106.20 \\
\hline & 1B & 12.66 & 36.0 & 3.67 & 37.0 & 4.12 & 52.2 & 1069.20 \\
\hline & $2 A$ & 12.96 & 41.0 & 5.64 & 40.5 & 5.41 & 56.2 & 1145.20 \\
\hline & $2 \mathrm{~B}$ & 13.28 & 40.5 & 5.09 & 42.0 & 5.77 & 54.2 & 1158.20 \\
\hline & $3 A$ & 13.31 & 46.3 & 7.67 & 47.0 & 8.01 & 54.2 & 1152.20 \\
\hline & 38 & 13.27 & 47.5 & 8.28 & 47.0 & 8.05 & 51.2 & 1186.20 \\
\hline & $4 \mathrm{~A}$ & 13.10 & 53.0 & 10.94 & 52.8 & 10.83 & 54.2 & 1161.20 \\
\hline & $4 \mathrm{~B}$ & 13.01 & 52.5 & 10.80 & 53.5 & 11.26 & 52.2 & 1160.20 \\
\hline \multirow[t]{8}{*}{ Day 151} & $1 \mathrm{~A}$ & 12.64 & 36.0 & 3.69 & 35.5 & 3.46 & 55.2 & 1161.40 \\
\hline & $1 \mathrm{~B}$ & 12.66 & 36.0 & 3.67 & 36.5 & 3.90 & 50.2 & 1119.40 \\
\hline & $2 A$ & 12.96 & 39.5 & 4.96 & 40.8 & 5.52 & 56.2 & 1201.40 \\
\hline & 28 & 13.28 & 41.5 & 5.54 & 42.0 & 5.77 & 54.2 & 1212.40 \\
\hline & $3 \mathrm{~A}$ & 13.31 & 46.3 & 7.67 & 47.3 & 8.12 & 53.2 & 1205.40 \\
\hline & $3 \mathrm{~B}$ & 13.27 & 46.5 & 7.82 & 47.5 & 8.28 & 55.2 & 1241.40 \\
\hline & $4 \mathrm{~A}$ & 13.10 & 52.0 & 10.49 & 52.5 & 10.71 & 58.2 & 1219.40 \\
\hline & $4 \mathrm{~B}$ & 13.01 & 52.5 & 10.80 & 52.0 & 10.58 & 51.2 & 1211.40 \\
\hline \multirow[t]{8}{*}{ Day 158} & $1 \mathrm{~A}$ & 12.64 & 31.0 & 1.42 & 32.3 & 1.99 & 50.2 & 1211.60 \\
\hline & $1 \mathrm{~B}$ & 12.66 & 32.5 & 2.08 & 33.8 & 2.65 & 46.2 & 1165.60 \\
\hline & $2 A$ & 12.96 & 35.0 & 2.92 & 36.8 & 3.71 & 48.2 & 1249.60 \\
\hline & $2 B$ & 13.28 & 36.0 & 3.05 & 37.5 & 3.73 & 48.2 & 1260.60 \\
\hline & $3 \mathrm{~A}$ & 13.31 & 43.0 & 6.19 & 44.5 & 6.87 & 54.2 & 1259.60 \\
\hline & $3 \mathrm{~B}$ & 13.27 & 44.0 & 6.69 & 44.5 & 6.91 & 55.2 & 1296.60 \\
\hline & $4 \mathrm{~A}$ & 13.10 & 48.5 & 8.90 & 50.0 & 9.58 & 56.2 & 1275.60 \\
\hline & $4 \mathrm{~B}$ & 13.01 & 49.5 & 9.44 & 51.3 & 10.24 & 46.2 & 1257.60 \\
\hline \multirow[t]{8}{*}{ Day 165} & $1 \mathrm{~A}$ & 12.64 & 29.8 & 0.85 & 32.1 & 1.91 & 44.2 & 1255.80 \\
\hline & $1 \mathrm{~B}$ & 12.66 & 30.5 & 1.17 & 32.2 & 1.94 & 43.2 & 1208.80 \\
\hline & $2 \mathrm{~A}$ & 12.96 & 31.8 & 1.44 & 34.7 & 2.79 & 45.2 & 1294.80 \\
\hline & $2 \mathrm{~B}$ & 13.28 & 33.0 & 1.69 & 35.3 & 2.73 & 50.2 & 1310.80 \\
\hline & $3 \mathrm{~A}$ & 13.31 & 39.3 & 4.49 & 41.0 & 5.29 & 51.2 & 1310.80 \\
\hline & $3 \mathrm{~B}$ & 13.27 & 38.0 & 3.97 & 39.7 & 4.71 & 51.2 & 1347.80 \\
\hline & $4 \mathrm{~A}$ & 13.10 & 47.0 & 8.22 & 48.9 & 9.09 & 56.2 & 1331.80 \\
\hline & $4 \mathrm{~B}$ & 13.01 & 47.0 & 8.31 & 50.3 & 9.82 & 52.2 & 1309.80 \\
\hline \multirow[t]{8}{*}{ Day 172} & $1 \mathrm{~A}$ & 12.64 & 30.0 & 0.97 & 32.8 & 2.25 & 53.2 & 1309.00 \\
\hline & $1 B$ & 12.66 & 30.5 & 1.17 & 33.7 & 2.62 & 53.2 & 1262.00 \\
\hline & $2 A$ & 12.96 & 32.0 & 1.55 & 34.7 & 2.79 & 48.2 & 1343.00 \\
\hline & $2 B$ & 13.28 & 33.0 & 1.69 & 35.8 & 2.96 & 56.2 & 1367.00 \\
\hline & $3 \mathrm{~A}$ & 13.31 & 36.5 & 3.25 & 39.5 & 4.61 & 57.2 & 1368.00 \\
\hline & 38 & 13.27 & 35.5 & 2.83 & 38.1 & 4.03 & 53.2 & 1401.00 \\
\hline & $4 \mathrm{~A}$ & 13.10 & 43.5 & 6.63 & 46.9 & 8.18 & 55.2 & 1387.00 \\
\hline & $4 \mathrm{~B}$ & 13.01 & 44.3 & 7.06 & 46.6 & 8.13 & 50.2 & 1360.00 \\
\hline \multirow[t]{8}{*}{ Day 180} & $1 \mathrm{~A}$ & 12.64 & 29.8 & 0.89 & - & 2.47 & 61.8 & 1370.80 \\
\hline & 18 & 12.66 & 30.2 & 1.03 & - & 2.70 & 58.4 & 1320.40 \\
\hline & $2 A$ & 12.96 & 31.7 & 1.43 & - & 3.03 & 61.8 & 1404.80 \\
\hline & $2 \mathrm{~B}$ & 13.28 & 32.6 & 1.48 & - & 2.97 & 64.8 & 1431.80 \\
\hline & $3 A$ & 13.31 & 34.5 & 2.34 & - & 4.08 & 64.4 & 1432.40 \\
\hline & $3 \mathrm{~B}$ & 13.27 & 34.1 & 2.22 & - & 3.81 & 62.5 & 1463.50 \\
\hline & $4 \mathrm{~A}$ & 13.10 & 40.4 & 5.23 & - & 7.53 & 62.9 & 1449.90 \\
\hline & $4 \mathrm{~B}$ & 13.01 & 41.6 & 5.86 & - & 8.30 & 66.7 & 1426.70 \\
\hline
\end{tabular}


Phase 2 - Cumulative Sampling Losses

\begin{tabular}{|c|c|c|c|c|c|}
\hline \multirow[t]{2}{*}{ Reactor } & Initial Dry Mass & $\begin{array}{c}\text { Cumulative } \\
\text { Sample Mass } \\
\text { Collected } \\
\end{array}$ & $\begin{array}{l}\text { Cumulative Mass of } \\
\text { Compost Material } \\
\text { Removed for Sampling }\end{array}$ & $\begin{array}{l}\text { Cumulative Mass of } \\
\text { Compost Material } \\
\text { Removed for Sampling }\end{array}$ & $\begin{array}{l}\text { Total Compost } \\
\text { Material Removed } \\
\text { for Sampling }\end{array}$ \\
\hline & $(\mathrm{kg})$ & (g, wet basis) & (kg, wet basis) & $\left(\mathrm{kg}\right.$, dry basis $\left.{ }^{1}\right)$ & $(\%)$ \\
\hline $1 \mathrm{~A}$ & 4.542 & 1370.8 & 1.371 & 0.41 & $9.1 \%$ \\
\hline $1 \mathrm{~B}$ & 4.614 & 1320.4 & 1.320 & 0.40 & $8.6 \%$ \\
\hline $2 \mathrm{~A}$ & 5.829 & 1404.8 & 1.405 & 0.42 & $7.2 \%$ \\
\hline $2 \mathrm{~B}$ & 6.060 & 1431.8 & 1.432 & 0.43 & $7.1 \%$ \\
\hline $3 \mathrm{~A}$ & 8.667 & 1432.4 & 1.432 & 0.43 & $5.0 \%$ \\
\hline $3 \mathrm{~B}$ & 9.288 & 1463.5 & 1.464 & 0.44 & $4.7 \%$ \\
\hline $4 \mathrm{~A}$ & 9.684 & 1449.9 & 1.450 & 0.43 & $4.5 \%$ \\
\hline $4 \mathrm{~B}$ & 9.351 & 1426.7 & 1.427 & 0.43 & $4.6 \%$ \\
\hline
\end{tabular}

Notes:

1 - Dry basis calculation assumes target moisture content of $70 \%$ maintained throughout Phase 2 experiment. 
Phase 2 - \% Lignin (dry matter basis)

Feedstock Straw Samples:

\begin{tabular}{|c|c|}
\hline Sample Year & $\%$ Lignin \\
\hline 1997 & 12.9070 \\
\hline 1999 & 10.7592 \\
\hline 2000 & 10.4017 \\
\hline Average $=$ & 11.3560 \\
\hline
\end{tabular}

\begin{tabular}{|c|c|c|c|c|c|c|c|c|c|c|c|c|c|}
\hline \multirow{2}{*}{\begin{tabular}{|l|l}
\multicolumn{1}{c}{$\mathrm{Da}$} \\
Reactor & \\
\end{tabular}} & \multicolumn{13}{|l|}{ Day } \\
\hline & 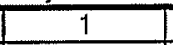 & 4 & 18 & 25 & 46 & 60 & 74 & 95 & 109 & 123 & 137 & 158 & 180 \\
\hline $1 \mathrm{a}$ & 11.3560 & 11.4143 & 15.6958 & 13.1259 & 16.5053 & 18.7770 & 17.1932 & 20.4162 & 17.1290 & 18.5357 & 16.58139 & 18.1158 & 19.3266 \\
\hline $1 b$ & 11.3560 & 11.5471 & 13.2472 & 14.1856 & 15.1916 & 19.0275 & 17.6036 & 19.3184 & 16.38613 & 16.6897 & 17.96692 & 17.9358 & 19.1240 \\
\hline Recipe 1 Mean & 11.3560 & 11.4807 & 14.4715 & 13.6558 & 15.8485 & 18.9023 & 17.3984 & 19.8673 & 16.7576 & 17.6127 & 17.2742 & 18.0258 & 19.2253 \\
\hline Recipe 1 St.Dev. & 0.0000 & 0.0939 & 1.7314 & 0.7493 & 0.9290 & & 0.2902 & 0.7763 & 0.5253 & 1.3053 & 0.9797 & 0.1273 & 0.1433 \\
\hline $2 \mathrm{a}$ & 11.3560 & 11.4614 & 17.6170 & 15.4702 & 14.1040 & 17.7486 & 14.7487 & 17.2671 & 16.05755 & 16.1799 & 17.19412 & 15.4811 & 16.7692 \\
\hline $2 b$ & 11.3560 & 13.1688 & 13.1455 & 13.9982 & 15.4710 & 15.1497 & 17.0399 & 17.7541 & 16.87615 & 15.1819 & 16.38949 & 15.8878 & 17.2145 \\
\hline Recipe 2 Mean & 11.3560 & 12.3151 & 15.3813 & 14.7342 & 14.7875 & 16.4492 & 15.8943 & 17.5106 & 16.4668 & 15.6809 & 16.7918 & 15.6845 & 16.9919 \\
\hline Recipe 2 St.Dev. & 0.0000 & 1.2073 & 3.1618 & 1.0409 & 0.9666 & 1.8377 & 1.6201 & 0.3444 & 0.5788 & 0.7057 & 0.5690 & 0.2875 & 0.3149 \\
\hline $3 a$ & 11.3560 & 12.7613 & 14.1692 & 15.5324 & 15.0185 & 17.7888 & 15.1296 & 20.8884 & 17.65214 & 16.2850 & 15.5137 & 15.9620 & 16.7574 \\
\hline $3 b$ & 11.3560 & 11.6456 & 14.0835 & 16.0110 & 13.0099 & 16.7841 & 15.7847 & 16.7180 & 15.62298 & 16.9073 & 14.14768 & 14.2057 & 16.8257 \\
\hline Recipe 3 Mean & 11.3560 & 12.2035 & 14.1264 & 15.7717 & 14.0142 & 17.2865 & 15.4572 & 18.8032 & 16.6376 & 16.5962 & 14.8307 & 15.0839 & 16.7916 \\
\hline Recipe 3 St.Dev. & 0.0000 & 0.7889 & 0.0606 & 0.3384 & 1.4203 & 0.7104 & 0.4632 & 2.9489 & 1.4348 & 0.4400 & 0.9659 & 1.2419 & 0.0483 \\
\hline $4 a$ & 11.3560 & 17.3534 & 14.3528 & 19.7206 & 15.2737 & 20.6696 & 22.0074 & 23.0535 & 21.26192 & 21.9263 & 19.45204 & 19.8067 & 20.8878 \\
\hline $4 b$ & 11.3560 & 15.8867 & 14.3345 & 18.5047 & 15.8610 & 22.3080 & 20.6993 & 24.1403 & 21.20286 & 23.8815 & 20.5493 & 19.1910 & 21.7822 \\
\hline Recipe 4 Mean & 11.3560 & 16.6201 & 14.3436 & 19.1127 & 15.5674 & 21.4888 & 21.3534 & 23.5969 & 21.2324 & 22.9039 & 20.0007 & 19.4988 & 21.3350 \\
\hline Recipe 4 St.Dev. & 0.0000 & 1.0371 & 0.0129 & 0.8598 & 0.4152 & 1.1585 & 0.9250 & 0.7685 & 0.0418 & 1.3825 & 0.7759 & 0.4353 & 0.6324 \\
\hline
\end{tabular}

Notes:

1) \% Lignin on Day 1 is an average of 1997, 1999, and 2000 straw samples. 
Phase 2 - \% Cellulose (dry matter basis)

Feedstock Straw Samples:

\begin{tabular}{|c|r|}
\hline Sample Year & \% Cellulose \\
\hline 1997 & 37.9829 \\
\hline 1999 & 42.6874 \\
\hline 2000 & 46.7332 \\
\hline Average $=$ & 42.4678 \\
\hline
\end{tabular}

\begin{tabular}{|c|c|c|c|c|c|c|c|c|c|c|c|c|c|}
\hline & 1 & 4 & 18 & 25 & 46 & 60 & 74 & 95 & 109 & 123 & 137 & 158 & 180 \\
\hline$\frac{\text { hea }}{1 a}$ & $\frac{1}{42.4678}$ & 46.9532 & 44.8771 & 43.1407 & 43.00945 & 28.7907 & 30.53626 & 30.5847 & 27.71977 & 31.38411 & 25.18726 & 32.88933 & 30.74052 \\
\hline $1 b$ & 42.4678 & 47.6324 & 43.5674 & 42.8494 & 37.55437 & 37.2934 & 36.42124 & 30.0080 & 33.9268 & 29.20402 & 29.48354 & 30.51168 & 29.13004 \\
\hline Recipe 1 Mean & 42.4678 & 47.2928 & 44.2222 & 42.9951 & 40.2819 & 33.0421 & 33.4787 & 30.2964 & 30.8233 & 30.2941 & 27.3354 & 31.7005 & 29.9353 \\
\hline Recipe 1 St.Dev. & 0.0000 & 0.4803 & 0.9261 & 0.2060 & 3.8573 & 6.0123 & 4.1613 & 0.4078 & 4.3890 & 1.5416 & 3.0379 & 1.6813 & 1.1388 \\
\hline $2 a$ & 42.4678 & 43.6582 & 49.1742 & 38.2447 & 28.33992 & 26.3036 & 27.78458 & 26.1357 & 26.52561 & 24.59233 & 29.07582 & 29.83968 & 29.98075 \\
\hline $2 \mathrm{~b}$ & 42.4678 & 41.2150 & 35.6721 & 32.3361 & 26.86412 & 25.7737 & 24.33605 & 23.8026 & 29.09582 & 27.57791 & 30.75548 & 29.63392 & 29.31946 \\
\hline Recipe 2 Mean & 42.4678 & 42.4366 & 42.4231 & 35.2904 & 27.6020 & 26.0387 & 26.0603 & 24.9692 & 27.8107 & 26.0851 & 29.9157 & 29.7368 & 29.6501 \\
\hline Recipe 2 St.Dev. & 0.0000 & 1.7276 & 9.5474 & 4.1780 & 1.0435 & 0.3747 & 2.4385 & 1.6498 & 1.8174 & 2.1111 & 1.1877 & 0.1455 & 0.4676 \\
\hline $3 a$ & 42.4678 & 45.5207 & 33.0984 & 30.6930 & 27.68992 & 21.4579 & 24.78055 & 26.9022 & 32.2431 & 28.58637 & 28.19131 & 32.10054 & 29.95205 \\
\hline $3 b$ & 42.4678 & 44.5225 & 34.1293 & 32.3799 & 21.14122 & 22.3412 & 24.78858 & 29.2476 & 25.36863 & 32.13284 & 26.60072 & 26.90856 & 31.69963 \\
\hline Recipe 3 Mean & 42.4678 & 45.0216 & 33.6139 & 31.5365 & 24.4156 & 21.8996 & 24.7846 & 28.0749 & 28.8059 & 30.3596 & 27.3960 & 29.5045 & 30.8258 \\
\hline Recipe 3 St.Dev. & 0.0000 & 0.7058 & 0.7290 & 1.1928 & 4.6306 & 0.6246 & 0.0057 & 1.6584 & 4.8610 & 2.5077 & 1.1247 & 3.6713 & 1.2357 \\
\hline $4 a$ & 42.4678 & 44.3841 & 32.6072 & 29.8230 & 27.59073 & 33.2311 & 33.49478 & 29.0015 & 30.52839 & 29.11668 & 26.36648 & 29.69443 & 29.65232 \\
\hline $4 b$ & 42.4678 & 42.0952 & 34.0030 & 29.7198 & 29.13363 & 31.2927 & 32.6666 & 30.8032 & 28.96986 & 30.57773 & 24.98755 & 29.0969 & 29.24467 \\
\hline Recipe 4 Mean & 42.4678 & 43.2397 & 33.3051 & 29.7714 & 28.3622 & 32.2619 & 33.0807 & 29.9024 & 29.7491 & 29.8472 & 25.6770 & 29.3957 & 29.4485 \\
\hline Recipe 4 St.Dev & 0.0000 & 1.6185 & 0.9870 & 0.0730 & 1.0910 & 1.3707 & 0.5856 & 1.2740 & 1.1020 & 1.0331 & 0.9751 & 0.4225 & 0.2883 \\
\hline
\end{tabular}

Recipe 4 St.Dev.

Notes:

1) \% Cellulose on Day 1 is an average of 1997,1999 , and 2000 straw samples. 
Phase 2 - \% Hemicellulose (dry matter basis)

Feedstock Straw Samples:
\begin{tabular}{|c|r|}
\hline Sample Year & $\begin{array}{c}\% \text { Hemi- } \\
\text { Cellulose }\end{array}$ \\
\hline 1997 & 20.6272 \\
\hline 1999 & 19.6638 \\
\hline 2000 & 18.6504 \\
\hline Average $=$ & 19.6471 \\
\hline
\end{tabular}

\begin{tabular}{|c|c|c|c|c|c|c|c|c|c|c|c|c|c|}
\hline Reactor & 1 & 4 & 18 & 25 & 46 & 60 & 74 & 95 & 109 & 123 & 137 & 158 & 180 \\
\hline 1a & 19.6471 & 19.2534 & \begin{tabular}{|l|}
13.0953129 \\
\end{tabular} & 9.8269 & 2.795827 & 2.5897 & 12.47508 & -1.6792 & -2.417961 & & -2.103817 & -13.19606 & -6.435227 \\
\hline $1 \mathrm{~b}$ & 19.6471 & 16.8534 & 17.3925574 & 11.6415 & 11.22109 & 2.3016 & 4.200056 & 4.5636 & 1.268458 & 5.770109 & -6.45685 & -6.445028 & $\begin{array}{r}-6.419516 \\
-6.4274\end{array}$ \\
\hline Recipe 1 Mean & 19.6471 & 18.0534 & \begin{tabular}{|l|}
15.2439 \\
\end{tabular} & 10.7342 & 7.0085 & 2.4457 & 8.3376 & 1.4422 & -0.5748 & 1.1523 & $\frac{-4.2803}{20791}$ & $\frac{-9.8205}{4.7737}$ & -6.4274 \\
\hline Recipe 1 St.Dev. & 0.0000 & 1.6971 & 3.0386 & 1.2831 & 5.9576 & 0.2037 & 5.8513 & 4.4143 & 2.6067 & 6.5305 & $\begin{array}{r}3.0781 \\
1369742\end{array}$ & $\begin{array}{r}\frac{4.7737 \mid}{-8.52393} \\
\end{array}$ & $\begin{array}{l}\quad 0.0111 \\
-2.782026\end{array}$ \\
\hline$\frac{2 a}{2 b}$ & 19.6471 & 15.9864 & 1.2283158 & 7.3593 & 10.23703 & 5.1820 & 5.605122 & \begin{tabular}{r|}
-1.6888 \\
58774
\end{tabular} & $\begin{array}{r}-4.21078 \\
-1061344\end{array}$ & $\begin{array}{l}6.023566 \\
2.966874\end{array}$ & $\begin{array}{l}1.369742 \\
-1.56257\end{array}$ & $\begin{array}{r}-5.52393 \\
-5.152862\end{array}$ & $\begin{array}{l}-2.782026 \\
-2.464633\end{array}$ \\
\hline $\begin{array}{l}2 b \\
\text { Recipe } 2 \text { Mean }\end{array}$ & 19.6471 & 17.5572 & 9.02026098 & 12.7611 & $\frac{4.511377}{73742}$ & 4.1171 & $\begin{array}{r}2.368538 \\
3.9868\end{array}$ & $\frac{-5.8774}{-3.7831}$ & $\frac{0.61344}{-7.41211}$ & 4.4952 & -0.0964 & \begin{tabular}{|l|}
-6.8384 \\
\end{tabular} & $\begin{array}{r}-2.404635 \\
-2.6233 \\
\end{array}$ \\
\hline $\begin{array}{l}\text { Recipe } 2 \text { Mean } \\
\text { Recipe } 2 \text { St.Dev. }\end{array}$ & 19.6471 & 16.7718 & \begin{tabular}{|l|}
5.1243 \\
55007
\end{tabular} & $\begin{array}{r}10.0602 \\
3.8196\end{array}$ & $\frac{7.3 / 42}{4.0486}$ & $\begin{array}{r}\frac{4.6496}{0.7530} \\
\end{array}$ & $\begin{array}{r}3.9868 \\
2.2886\end{array}$ & 2.9618 & 4.5274 & 2.1614 & 2.0735 & 2.3837 & 0.2244 \\
\hline Recipe 2 St.Dev. & 0.0000 & 1.1107 & $\begin{array}{r}5.5097 \\
15.191608\end{array}$ & $\begin{array}{r}3.8196 \\
14.4203\end{array}$ & $\begin{array}{r}4.0400 \\
9.552694\end{array}$ & $\begin{array}{r}0.7530 \\
2.9110\end{array}$ & 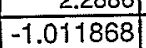 & -3.8696 & -8.036319 & -0.904961 & 3.464985 & -7.580948 & -3.39122 \\
\hline$\frac{3 a}{3 b}$ & 19.6471 & 18.5879 & $\begin{array}{l}15.4184608 \\
122915207\end{array}$ & $\frac{1.4 \angle 00}{12.7606}$ & 8.142306 & 0.7494 & 0.43696 & -13.0759 & -1.604789 & -10.27879 & 0.538108 & -4.315688 & 7.71191 \\
\hline Bb & 19.6471 & 17.2743 & $\frac{12.2915207}{13.8550}$ & 13.5905 & 8.8475 & $\frac{0.7434}{1.8302}$ & -0.2875 & -8.4728 & -4.8206 & \begin{tabular}{|l|}
-5.5919 \\
\end{tabular} & 2.0015 & -5.9483 & -5.5516 \\
\hline Recipe 3 Mean & 19.6471 & 17.9311 & $\frac{13.8550}{2.111}$ & 11736 & 0.9973 & $\frac{1.005}{1.5285}$ & 1.0245 & 6.5098 & 4.5478 & 6.6283 & 2.0696 & 2.3089 & 3.055 \\
\hline Recipe 3 St.Dev. & 0.0000 & 0.9289 & 2.2111 & $1.1 / 314$ & & $\frac{1.0<00}{-10582}$ & -3.692248 & -1.4494 & -3.17615 & -4.463249 & 3.385255 & -3.572675 & -4.75255 \\
\hline $4 a$ & 19.6471 & 5.4762 & 15.1694742 & $\frac{1.3717}{100355}$ & $\begin{array}{l}7.995301 \\
8287499\end{array}$ & & $\begin{array}{r}-2.09<240 \\
2.078843\end{array}$ & -1.7204 & & -1.415311 & 346244 & 1.114502 & -5.30288 \\
\hline $4 \mathrm{~b}$ & 19.6471 & 13.6789 & 10.7215661 & $\frac{10.0355}{5.6736}$ & $\frac{8.284999}{8.1414}$ & $\frac{2.5104}{0.7261}$ & $\begin{array}{c}2.0 .08843 \\
-0.8067\end{array}$ & $\frac{-1 . / 204}{-1.5849}$ & $\frac{30500}{0.5712}$ & $\frac{7.9393}{-2.9}$ & 4.8657 & -2.3436 & -5.0277 \\
\hline \begin{tabular}{|l} 
Recipe 4 Mean \\
Recipe 4 St.Dev
\end{tabular} & $\frac{19.6471}{0.0000}$ & 9.5776 & $\begin{array}{r}12.9455 \\
3.1451\end{array}$ & $\frac{5.6736}{6.1687}$ & & $\frac{0.1234}{2.5234}$ & 4.0808 & 0.1916 & 5.2996 & 2.1552 & 2.0937 & 1.7382 & 0.389 \\
\hline Recipe 4 & 0.0000 & & & & & & & & & & & & \\
\hline
\end{tabular}

1) \% Hemicellulose on Day 1 is an average of 1997, 1999, and 2000 straw samples. 
Phase 2 - g Lignin / g Ash (dry matter basis)

Feedstock Straw Samples:
\begin{tabular}{|c|r|r|r|}
\hline Sample Year & \% lignin & \%FS & g lignin / g ash \\
\hline 1997 & 12.907 & 12.26 & 1.0528 \\
\hline 1999 & 10.7592 & 12.29 & 0.8754 \\
\hline 2000 & 10.4017 & 9.88 & 1.0528 \\
\hline Average & 11.3560 & 11.48 & 0.9937 \\
\hline
\end{tabular}

\begin{tabular}{|c|c|c|c|c|c|c|c|c|c|c|c|c|c|}
\hline & 1 & 4 & 1 & 25 & & 6 & 74 & 95 & 109 & 123 & 137 & 158 & 180 \\
\hline \begin{tabular}{|l} 
Reactor \\
$1 \mathrm{a}$
\end{tabular} & $\frac{1}{0.9937}$ & 0.9985 & 1.1596 & 0.9176 & 0.9501 & 0.8576 & 0.7192 & 0.7469 & 0.5616 & 0.5397 & 0.4535 & 0.4650 & 0.5048 \\
\hline $1 \mathrm{~b}$ & 0.9937 & 1.0241 & 1.0491 & 1.0824 & 0.9681 & 0.9757 & 0.8626 & 0.8232 & 0.5876 & 0.5893 & 0.5588 & 0.4959 & 0.5217 \\
\hline Recipe 1 Mean & 0.9937 & 1.0113 & 1.1044 & 1.0000 & 0.9591 & 0.9167 & 0.7909 & 0.7850 & 0.5746 & 0.5645 & 0.5061 & 0.4805 & 0.5132 \\
\hline Recipe 1 St.Dev. & 0.0000 & 0.0181 & 0.0782 & 0.1165 & 0.0127 & 0.0835 & 0.1014 & 0.0539 & 0.0183 & 0.0351 & 0.0745 & 0.0218 & 0.0120 \\
\hline $2 a$ & 0.9937 & 1.0383 & 1.1785 & 0.9027 & 0.6510 & 0.7391 & 0.5716 & 0.5549 & 0.4657 & 0.4443 & 0.4542 & 0.3990 & 0.4273 \\
\hline $2 b$ & 0.9937 & 1.0096 & 0.8019 & 0.7482 & 0.6079 & 0.5400 & 0.5520 & 0.5021 & 0.4528 & 0.3974 & 0.4262 & 0.3932 & 0.4330 \\
\hline Recipe 2 Mean & 0.9937 & 1.0240 & 0.9902 & 0.8255 & 0.6295 & 0.6396 & 0.5618 & 0.5285 & 0.4592 & 0.4209 & 0.4402 & 0.3961 & 0.4302 \\
\hline Recipe 2 St.Dev. & 0.0000 & 0.0203 & 0.2663 & 0.1093 & 0.0305 & 0.1408 & 0.0138 & 0.0974 & 0.0091 & 0.0331 & 0.0198 & 0.0041 & 0.0040 \\
\hline 3a & 0.9937 & 1.0178 & 0.7769 & 0.7475 & 0.5655 & 0.5996 & 0.4580 & 0.5710 & 0.4705 & 0.4130 & 0.3876 & 0.3867 & 0.4029 \\
\hline $3 b$ & 0.9937 & 0.8336 & 0.7869 & 0.7879 & 0.4692 & 0.5233 & 0.4486 & 0.4332 & 0.4003 & 0.4176 & 0.3408 & 0.3366 & 0.4003 \\
\hline Recipe 3 Mean & 0.9937 & 0.9257 & 0.7819 & 0.7677 & 0.5174 & 0.5615 & 0.4533 & 0.5021 & 0.4354 & 0.4153 & 0.3642 & 0.3617 & 0.4016 \\
\hline Recipe 3 St.Dev. & 0.0000 & 0.1303 & 0.0071 & 0.0286 & 0.0681 & 0.0540 & 0.0066 & 0.0975 & 0.0496 & 0.0032 & 0.0331 & 0.0355 & 0.0018 \\
\hline$\frac{14}{4 a}$ & 0.9937 & 1.2703 & 0.8868 & 1.1387 & 0.7079 & 0.8897 & 0.8603 & 0.8003 & 0.6903 & 0.6717 & 0.5781 & 0.5633 & 0.5792 \\
\hline $4 \mathrm{~b}$ & 0.9937 & 1.1285 & 0.8850 & 1.0946 & 0.7964 & 1.0365 & 0.8830 & 0.9601 & 0.7746 & 0.7979 & 0.6475 & 0.5641 & 0.6264 \\
\hline$\frac{74}{\operatorname{Beci}}$ & 0.9937 & 1.1994 & 0.8859 & 1.1166 & 0.7522 & 0.9631 & 0.8716 & 0.8802 & 0.7325 & 0.7348 & 0.6128 & 0.5637 & 0.6028 \\
\hline & 0.0000 & 0.1002 & 0.0013 & 0.0312 & 0.0626 & 0.1038 & 0.0161 & 0.1130 & 0.0596 & 0.0893 & 0.0491 & 0.0006 & 0.0334 \\
\hline
\end{tabular}

Notes:

1) $\%$ Lignin on Day $\uparrow$ is an average of 1997,1999 , and 2000 straw samples. 
Phase 2 - $\mathrm{g}$ Cellulose / $\mathrm{g}$ Ash (dry matter basis)

Feedstock Straw Samples:
\begin{tabular}{|c|r|r|r|}
\hline Sample Year & $\%$ Cellulose & \%FS & $\begin{array}{c}\text { g Cellulase } \\
\text { /g Ash }\end{array}$ \\
\hline 1997 & 37.9829 & 12.26 & 3.0981 \\
\hline 1999 & 42.6874 & 12.29 & 3.4733 \\
\hline 2000 & 46.7332 & 9.88 & 4.7301 \\
\hline Average = & 42.4678 & 11.48 & 3.7672 \\
\hline
\end{tabular}

\begin{tabular}{|c|c|c|c|c|c|c|c|c|c|c|c|c|c|}
\hline Reactor & 1 & 4 & 18 & 25 & 46 & 60 & 74 & 95 & 109 & 123 & 137 & 158 & 180 \\
\hline $1 \mathrm{a}$ & 3.7672 & 4.1074 & 3.3156 & 3.0159 & 2.4758 & 1.3149 & 1.2773 & 1.1189 & 0.9089 & 0.9138 & 0.6888 & 0.8442 & 0.8029 \\
\hline $1 \mathrm{~b}$ & 3.7672 & 4.2245 & 3.4502 & 3.2694 & 2.3933 & 1.9124 & 1.7846 & 1.2787 & 1.2165 & 1.0312 & 0.9169 & 0.8436 & 0.7947 \\
\hline Recipe 1 Mean & 3.7672 & 4.1660 & 3.3829 & 3.1427 & 2.4345 & 1.6137 & 1.5310 & 1.1988 & 1.0627 & 0.9725 & 0.8029 & 0.8439 & 0.7988 \\
\hline Recipo 1 St.Dev. & 0.0000 & 0.0828 & 0.0952 & 0.1793 & 0.0584 & 0.4225 & 0.3587 & 0.1130 & 0.2175 & 0.0830 & 0.1613 & 0.0004 & 0.0058 \\
\hline $2 a$ & 3.7672 & 3.9552 & 3.2894 & 2.2317 & 1.3082 & 1.0954 & 1.0767 & 0.8399 & 0.7693 & 0.6753 & 0.7680 & 0.7691 & 0.7639 \\
\hline $2 \mathrm{~b}$ & 3.7672 & 3.1598 & 2.1760 & 1.7283 & 1.0556 & 0.9187 & 0.7884 & 0.6731 & 0.7806 & 0.7220 & 0.7999 & 0.7334 & 0.7375 \\
\hline Recipe 2 Mean & 3.7672 & 3.5575 & 2.7327 & 1.9800 & 1.1819 & 1.0071 & 0.9326 & 0.7565 & 0.7749 & 0.6986 & 0.7839 & 0.7512 & 0.7507 \\
\hline Recipe 2 St.Dev. & 0.0000 & 0.5625 & $\begin{array}{l}0.7873 \\
\end{array}$ & 0.3559 & 0.1786 & 0.1250 & 0.2039 & 0.1180 & 0.0080 & 0.0330 & 0.0225 & 0.0252 & 0.0187 \\
\hline $3 a$ & 3.7672 & 3.6307 & 1.8148 & 1.4770 & 1.0427 & 0.7233 & 0.7501 & 0.7354 & 0.8595 & 0.7250 & 0.7043 & 0.7777 & 0.7201 \\
\hline $3 b$ & 3.7672 & 3.1870 & 1.9070 & 1.5934 & 0.7625 & 0.6966 & 0.7045 & 0.7578 & 0.6501 & 0.7936 & 0.6408 & 0.6376 & 0.7541 \\
\hline Recipe 3 Mean & 3.7672 & 3.4089 & 1.8609 & 1.5352 & 0.9026 & 0.7099 & 0.7273 & 0.7466 & 0.7548 & 0.7593 & 0.6726 & 0.7076 & 0.7371 \\
\hline Recipo 3 St.Dev. & 0.0000 & 0.3137 & 0.0652 & 0.0823 & 0.1981 & 0.0189 & 0.0322 & 0,0159 & 0.1481 & 0.0485 & 0.0449 & 0.0991 & 0.0241 \\
\hline $4 a$ & 3.7672 & 3.2489 & 2.0147 & 1.7221 & 1.2787 & 1.4305 & 1.3093 & 1.0067 & 0.9912 & 0.8920 & 0.7835 & 0.8444 & 0.8223 \\
\hline $4 \mathrm{~b}$ & 3.7672 & 2.9903 & 2.0992 & 1.7580 & 1.4629 & 1.4540 & 1.3935 & 1.2251 & 1.0584 & 1.0217 & 0.7874 & 0.8552 & 0.8411 \\
\hline Recipe 4 Mean & 3.7672 & 3.1196 & 2.0570 & 1.7400 & 1.3708 & 1.4422 & 1.3514 & 1.1159 & 1.0248 & 0.9568 & 0.7855 & 0.8498 & 0.8317 \\
\hline Recipe 4 St.Dev. & 0.0000 & 0.1829 & 0.0598 & 0.0254 & 0.1303 & 0.0166 & 0.0595 & 0.1544 & 0.0475 & 0.0917 & 0.0027 & 0.0076 & 0.0133 \\
\hline
\end{tabular}

Recipe

1) \% Cellulose on Day 1 is an average of 1997, 1999, and 2000 straw samples. 
Phase 2 - g Hemicellulose / g Ash (dry matter basis)

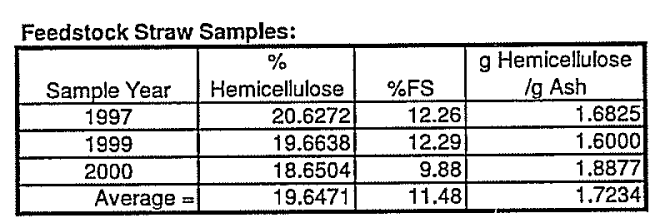

\begin{tabular}{|c|c|c|c|c|c|c|c|c|c|c|c|c|c|}
\hline Reactor & $\frac{1}{1}$ & 4 & 18 & 25 & 46 & 60 & 74 & 95 & 109 & 123 & 137 & 158 & 180 \\
\hline $1 \mathrm{a}$ & 1.7234 & 1.6843 & 0.9675 & 0.6870 & 0.1609 & 0.1183 & 0.5218 & -0.0614 & -0.0793 & -0.1009 & -0.0575 & -0.3387 & -0.1681 \\
\hline $1 \mathrm{~b}$ & 1.7234 & 1.4947 & 1.3773 & 0.8883 & 0.7151 & 0.1180 & 0.2058 & 0.1945 & 0.0455 & 0.2037 & -0.2008 & -0.1782 & -0.1751 \\
\hline Recipe 1 Mean & 1.7234 & 1.5895 & 1.1724 & 0.7876 & 0.4380 & 0.1182 & 0.3638 & 0.0665 & -0.0169 & 0.0514 & $\begin{array}{l}-0.1292 \\
\end{array}$ & -0.2585 & -0.1716 \\
\hline Recipe 1 St.Dev. & 0.0000 & 0.1340 & 0.2898 & 0.1423 & 0.3918 & 0.0002 & 0.2235 & 0.1809 & 0.0882 & 0.2154 & 0.1013 & 0.1135 & 0.0050 \\
\hline $2 a$ & 1.7234 & 1.4483 & 0.0822 & 0.4294 & 0.4725 & 0.2158 & 0.2172 & -0.0543 & -0.1221 & 0.1654 & 0.0362 & -0.2197 & -0.0709 \\
\hline $2 b$ & 1.7234 & 1.3460 & 0.5502 & 0.6821 & 0.1773 & 0.1468 & 0.0767 & -0.1662 & -0.2847 & 0.0777 & -0.0406 & -0.1275 & -0.0620 \\
\hline Recipe 2 Mean & 1.7234 & 1.2858 & 0.3337 & 0.6137 & 0.3941 & 0.2188 & 0.1740 & -0.1487 & -0.2773 & 0.1602 & -0.0033 & -0.2215 & -0.0804 \\
\hline Recipe 2 St.Dev. & 0.0000 & 0.0723 & 0.3310 & 0.1786 & 0.2088 & 0.0488 & 0.0993 & 0.0791 & 0.1150 & 0.0620 & 0.0543 & 0.0652 & 0.0063 \\
\hline 3a & 1.7234 & 1.4826 & 0.8454 & 0.6939 & 0.3597 & 0.0981 & -0.0306 & -0.1058 & -0.2142 & -0.0230 & 0.0866 & -0.1837 & -0.0815 \\
\hline $3 \mathrm{~b}$ & 1.7234 & 1.2365 & 0.6868 & 0.6280 & 0.2937 & 0.0234 & 0.0124 & -0.3388 & -0.0411 & -0.2538 & 0.0130 & -0.1023 & -0.1835 \\
\hline Recipe 3 Mean & 1.7234 & 1.3595 & 0.7661 & 0.6609 & 0.3267 & 0.0607 & -0.0091 & -0.2223 & -0.1277 & -0.1384 & 0.0498 & -0.1430 & -0.1325 \\
\hline Recipe 3 St.Dev, & 0.0000 & 0.1740 & 0.1121 & 0.0467 & 0.0467 & 0.0529 & 0.0304 & 0.1648 & 0.1224 & 0.1633 & 0.0520 & 0.0576 & 0.0721 \\
\hline $4 \mathrm{a}$ & 1.7234 & 0.4009 & 0.9373 & 0.0757 & 0.3705 & -0.0456 & -0.1443 & -0.0503 & -0.1031 & -0.1367 & 0.1006 & -0.1016 & -0.1318 \\
\hline$\frac{7 a}{4 b}$ & 1.7234 & 0.9717 & 0.6619 & 0.5936 & 0.4161 & 0.1166 & 0.0887 & -0.0684 & 0.1578 & -0.0473 & 0.2000 & -0.0328 & -0.1525 \\
\hline Recipe 4 Mean & 1.7234 & 0.6863 & 0.7996 & 0.3347 & 0.3933 & 0.0355 & -0.0278 & -0.0594 & 0.0273 & -0.0920 & 0.1503 & -0.0672 & -0.1421 \\
\hline Recipe 4 St.Dev. & 0.0000 & 0.4036 & 0.1947 & 0.3662 & 0.0322 & 0.1147 & 0.1648 & 0.0128 & 0.1845 & 0.0632 & 0.0703 & 0.0487 & 0.0146 \\
\hline
\end{tabular}

1) \% Hemicellulose on Day 1 is an average of 1997, 1999, and 2000 straw samples.

With correction to eliminate negative hemicellulose results:

\begin{tabular}{|c|c|c|c|c|c|c|c|c|c|c|c|c|c|}
\hline Reactor & & 4 & 18 & 25 & 46 & 60 & 74 & 95 & 109 & 123 & 137 & 158 & 180 \\
\hline $1 \mathrm{a}$ & 1.7234 & 1.6843 & 0.9675 & 0.6870 & 0.1609 & 0.1183 & 0.5218 & 0.0000 & 0.0000 & 0.0000 & 0.0000 & 0.0000 & 0.0000 \\
\hline $1 \mathrm{~b}$ & 1.7234 & 1.4947 & 1.3773 & 0.8883 & 0.7151 & 0.1180 & 0.2058 & 0.1945 & 0.0455 & 0.2037 & 0.0000 & 0.0000 & 0.0000 \\
\hline Recipe 1 Mean & 1.7234 & 1.5895 & 1.1724 & 0.7876 & 0.4380 & 0.1182 & 0.3638 & 0.0972 & 0.0227 & 0.0000 & & & \\
\hline Recipe I St.Dev. & 0.0000 & 0.1340 & 0.2898 & 0.1423 & 0.3918 & 0.0002 & 0.2235 & 0.1375 & 0.0322 & 0.1441 & & & \\
\hline $2 a$ & 1.7234 & 1.4483 & 0.0822 & 0.4294 & 0.4725 & 0.2158 & 0.2172 & 0.0000 & 0.0000 & 0.1654 & 0.0362 & 0.0000 & 0.0000 \\
\hline $2 \mathrm{~b}$ & 1.7234 & 1.3460 & 0.5502 & 0.6821 & 0.1773 & 0.1468 & 0.0767 & 0.0000 & 0.0000 & 0.0777 & 0.0000 & 0.0000 & 0.0000 \\
\hline Recipe 2 Mean & 1.7234 & 1.3972 & 0.3162 & 0.5558 & 0.3249 & 0.1813 & 0.1470 & 0.0000 & & & & & \\
\hline Recipe 2 St.Dev. & 0.0000 & 0.0723 & 0.3310 & 0.1786 & 0.2088 & 0.0488 & 0.0993 & 0.0000 & & & & & \\
\hline $3 a$ & 1.7234 & 1.4826 & 0.8454 & 0.6939 & 0.3597 & 0.0981 & 0.0000 & 0.0000 & 0.0000 & 0.0000 & 0.0866 & 0.0000 & 0.0000 \\
\hline $3 \mathrm{~b}$ & 1.7234 & 1.2365 & 0.6868 & 0.6280 & 0.2937 & 0.0234 & 0.0124 & 0.0000 & 0.0000 & 0.0000 & 0.0130 & 0.0000 & 0.0000 \\
\hline Recipe 3 Mean & 1.7234 & 1.3595 & 0.7661 & 0.6609 & 0.3267 & 0.0607 & 0.0062 & 0.0000 & & & & & \\
\hline Recipe 3 St.Dev. & 0.0000 & 0.1740 & 0.1121 & 0.0467 & 0.0467 & 0.0529 & 0.0088 & 0.0000 & & & & & \\
\hline $4 a$ & 1.7234 & 0.4009 & 0.9373 & 0.0757 & 0.3705 & 0.0000 & 0.0000 & 0.0000 & 0.0000 & 0.0000 & 0.1006 & 0.0000 & 0.0000 \\
\hline $4 b$ & 1.7234 & 0.9717 & 0.6619 & 0.5936 & 0.4161 & 0.1166 & 0.0887 & 0.0000 & 0.1578 & 0.0000 & 0.2000 & 0.0000 & 0.0000 \\
\hline Recipe 4 Mean & 1.7234 & 0.6863 & 0.7996 & 0.3347 & 0.3933 & 0.0583 & 0.0443 & 0.0000 & & & & & \\
\hline Recipe 4 St.Dev. & 0.0000 & 0.4036 & 0.1947 & 0.3662 & 0.0322 & 0.0825 & 0.0627 & 0.0000 & & & & & \\
\hline
\end{tabular}




\section{Phase 2- Temperature Measurements}

\begin{tabular}{|c|c|c|c|c|c|c|c|c|c|c|c|c|c|c|c|c|c|c|c|c|c|c|c|c|}
\hline & & $\sqrt{3}$ & 4 & 7 & 8 & 10 & 14 & 16 & 17 & 18 & 19 & & & & 32 & 33 & 34 & 35 & 37 & 39 & 41 & 46 & 53 & 56 \\
\hline $1 \mathrm{~A}$ & 49.0 & 52.5 & 50.5 & 47.5 & 48.5 & 48.0 & 47.0 & 47.5 & 47.0 & 47.0 & 47.0 & 45.5 & 48.0 & 45.0 & 45.0 & 44.0 & 44.0 & 44.0 & 45.0 & 44.0 & 45.0 & 47.0 & 47.0 & \\
\hline $1 \mathrm{~B}$ & 49. & 53.0 & 48.5 & & 49.5 & 46.5 & 46 & 46.0 & 47.0 & 45 & 43.0 & 45.0 & & & & & & & & & 46.0 & & 3.0 & 5 \\
\hline $2 A$ & 51. & 54.0 & 53. & & & 48 & 49. & 50.0 & 50.0 & 48. & 5.5 & & 50 & 47 & 46.5 & & & & & & & & & 7 \\
\hline $2 \mathrm{~B}$ & 54. & 58.0 & 54.5 & 49.2 & 50.0 & 52.0 & 50.0 & 50.0 & 49.5 & 51. & 50.1 & 48.5 & 50 & 47. & 47.0 & 46 & 47 & & & & & & 5.0 & 45. \\
\hline $3 A$ & 52. & 54.7 & 56.3 & 52.3 & 55.3 & & 51.5 & 50.0 & 50.5 & 51. & 50.5 & 51.0 & 51. & 46 & 48.0 & 45 & & & 47 & & & 50 & 47.0 & 46. \\
\hline $3 B$ & 50. & 55.3 & 57. & 50 & 52.0 & 52.7 & 53.0 & 51.0 & 51.5 & 51. & 50. & 51 & 51 & & 48.5 & 47.5 & 50 & 48. & 49.0 & 47.5 & & & & 44. \\
\hline$\overline{4 A}$ & 45 & 50.0 & 52. & 49.0 & 51.7 & 51.7 & 51.5 & 51. & 50.0 & 50.5 & 49.0 & 51 & 51 & 47 & 46.0 & 45 & & & & & & & & 46. \\
\hline & 45 & 0 & 51.3 & 49.3 & 50.3 & 49.3 & 50.5 & 49.5 & 49.5 & 49.5 & 49.0 & 50.0 & 49.0 & 46.0 & 46.0 & 44.0 & 47.0 & 47.0 & 45.5 & 46.5 & 46.5 & 47.5 & 46.0 & 45. \\
\hline
\end{tabular}

On Recipe Basis:

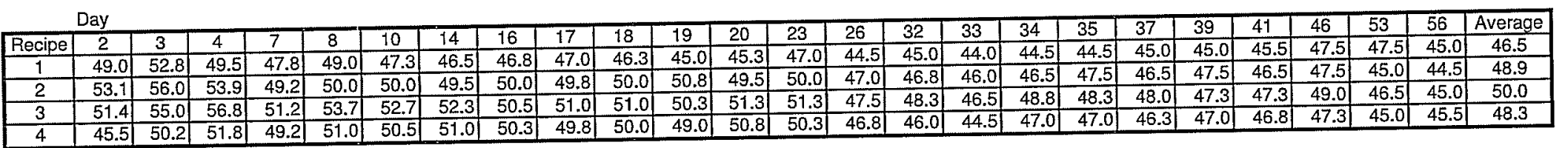

Note:

1) Temperatures recorded in ${ }^{\circ} \mathrm{C}$. 
Phase 2 - Oxygen Measurements

\begin{tabular}{|c|c|c|c|c|c|c|c|c|c|c|}
\hline \multicolumn{8}{|c|}{ Day } & Reactor & \multicolumn{2}{|c|}{ Recipe } \\
\hline Recipe & 13 & 14 & 16 & 17 & 18 & 19 & 20 & (Avg.) & (Avg.) & (St.Dev.) \\
\hline $1 \mathrm{~A}$ & NM & 17.5 & 18.5 & 18.5 & 17.0 & 17.5 & 19.0 & 18.0 & & \\
\hline $1 \mathrm{~B}$ & 16.5 & 18.0 & 18.0 & 18.5 & 13.5 & 15.0 & 19.0 & 17.0 & 17.5 & 0.8 \\
\hline $2 \mathrm{~A}$ & NM & 15.0 & 14.5 & 14.0 & 14.0 & 13.5 & 14.5 & 14.3 & & 19 \\
\hline $2 \mathrm{~B}$ & 13.5 & 13.5 & 10.0 & 10.5 & 10.0 & 14.0 & 11.5 & 11.6 & 12.9 & 1.9 \\
\hline $3 A$ & 12.0 & 8.5 & 11.5 & 10.0 & 7.0 & 12.0 & 10.5 & 9.9 & 10 & 01 \\
\hline $3 \mathrm{~B}$ & 10.6 & 8.5 & 10.0 & 7.5 & 8.0 & 13.5 & 13.0 & 10.1 & 10.0 & 0.1 \\
\hline $4 \mathrm{~A}$ & 9.3 & 7.5 & 9.5 & 9.0 & 8.5 & 7.5 & 8.0 & 8.3 & 80 & 08 \\
\hline $4 \mathrm{~B}$ & 13.3 & 9.0 & 11.0 & 9.0 & 9.5 & 8.5 & 10.0 & 9.5 & 0.9 & 0.0 \\
\hline
\end{tabular}

$\mathrm{NM}$ - Oxygen not measured on this day due to moisture content adjustment Notes:

1) Oxygen level recorded for each reactor represents average from all three sampling ports. 
Phase 2 - Finished Compost TKN Analysis

Test Date: February 11-15, 2003

Standards:

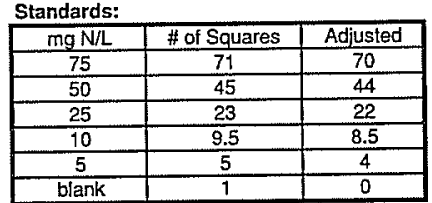

Conversion Equation: \# squares/ $0.9132=\mathrm{mg} \mathrm{N} / \mathrm{L}$

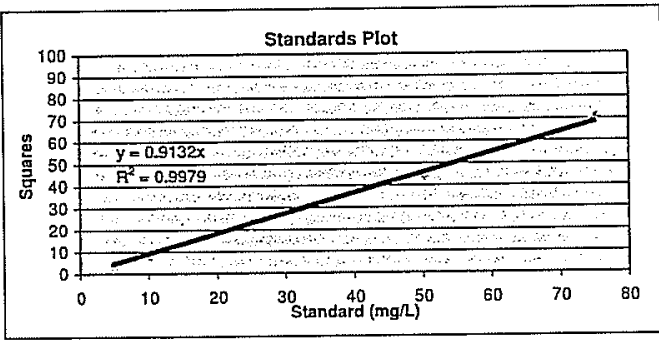

\begin{tabular}{|c|c|c|c|c|c|c|c|c|c|c|c|}
\hline & Sarnple Size & \# of Squares & $\mathrm{mgN}$ & $\mathrm{N}$ in Sample & $\% \mathrm{~N}$ & $\mathrm{gN} / \mathrm{g}$ Ash & $\begin{array}{c}\text { Initial N in } \\
\text { Peactor }\end{array}$ & $\begin{array}{l}\text { Final N in } \\
\text { Reactor }\end{array}$ & N Loss & $O C(d b)$ & $\begin{array}{c}\text { Finished } \\
\mathrm{C}: \mathrm{N}\end{array}$ \\
\hline Recipe & (mg) & & & $(\mathrm{mg})$ & (dry basis) & (dry basis) & (g, dry basis) & (g) & $(\%$, dry basis $)$ & (\%, dry basis) & \\
\hline \multirow{2}{*}{ 1a } & 100 & 43 & 47.09 & 2.354 & 2.35 & 0.061 & 3450 & 2260 & $34.50 \%$ & 34.28 & \\
\hline & 100 & 47 & 51.47 & 2.573 & 2.57 & 0.067 & 3450 & 2470 & $28.40 \%$ & 34.28 & \\
\hline & 100 & 50 & 54.75 & 2.738 & 2.74 & 0.071 & 3450 & 2628 & $23.83 \%$ & 34.28 & \\
\hline \multirow{2}{*}{$1 \mathrm{~b}$} & 100 & 49 & 53.66 & 2.683 & 2.68 & 0.073 & 3504 & 2951 & $15.77 \%$ & 35.19 & \\
\hline & $\frac{100}{100}$ & 49 & 53.66 & 2.683 & 2.68 & 0.073 & 3504 & 2951 & $15.77 \%$ & 35.19 & \\
\hline \multirow{2}{*}{\multicolumn{5}{|c|}{$\begin{array}{l}\text { Recipe } 1 \text { Mean } \\
\text { Recipe } 1 \text { StDev }\end{array}$}} & $\frac{2.46}{2.58}$ & $\begin{array}{l}0.067 \\
0.069\end{array}$ & $\frac{3504}{3477}$ & $\frac{2710}{2662}$ & $\frac{22.64 \%}{23.44 \%}$ & $\begin{array}{r}35.19 \\
34.74\end{array}$ & 13.5 \\
\hline & & & & & 0.15 & 0.005 & $\frac{29}{29}$ & 272 & 0.073 & & \\
\hline \multirow[t]{3}{*}{$2 a$} & 100 & 52 & 56.94 & 2.847 & 285 & 0.073 & 4431 & 4271 & $3.61 \%$ & 33.75 & \\
\hline & 100 & 53 & 58.04 & 2.902 & 2.90 & 0.074 & 4431 & 4353 & $1.76 \%$ & 33.75 & \\
\hline & 100 & 56 & 61.32 & 3.066 & 3.07 & 0.078 & 4431 & 4599 & $-3.80 \%$ & 33.75 & \\
\hline \multirow[t]{3}{*}{$2 b$} & 100 & 55 & 60.23 & 3.011 & 3.01 & 0.076 & 4606 & 4939 & $-7.23 \%$ & 33.47 & \\
\hline & 100 & 57 & 62.42 & 3.121 & 3.12 & 0.078 & 4606 & 5118 & $-11.13 \%$ & 33.47 & \\
\hline & 100 & 58 & 63.51 & 3.176 & 3.18 & 0.080 & 4606 & 5208 & $-13.08 \%$ & 33.47 & \\
\hline \multirow{2}{*}{\multicolumn{5}{|c|}{$\frac{\text { Recipe } 2 \text { Mean }}{\text { Recipe } 2 \text { St.Dev. }}$}} & 3.02 & 0.076 & 4518 & 4748 & $-5.09 \%$ & 33.61 & 11.1 \\
\hline & & & & & 0.13 & 0.003 & 96 & 398 & & & \\
\hline \multirow[t]{3}{*}{$3 a$} & 100 & 57 & 62.42 & 3.121 & 3.12 & 0.075 & 6589 & 6991 & $-6.09 \%$ & 32.45 & \\
\hline & 100 & 63 & 68.99 & 3.449 & 3.45 & 0.083 & 6589 & 7727 & $-17.26 \%$ & 32.45 & \\
\hline & 100 & 60 & 65.70 & 3.285 & 3.29 & 0.079 & 6589 & 7359 & $-11.68 \%$ & 32.45 & \\
\hline \multirow[t]{3}{*}{$3 b$} & 100 & 65 & 71.18 & 3.559 & 3.56 & 0.085 & 7050 & 8435 & $-19.46 \%$ & 32.2 & \\
\hline & 100 & 67 & 73.37 & 3.668 & 3.67 & 0.087 & 7060 & 8694 & $-23.14 \%$ & 32.2 & \\
\hline & 100 & 63 & 68.99 & 3.449 & 3.45 & 0.082 & 7060 & 8175 & $-15.79 \%$ & 32.2 & \\
\hline \multirow{2}{*}{\multicolumn{5}{|c|}{$\frac{\text { Recipe } 3 \text { Mean }}{\text { Recipe } 3 \text { St.Dev. }}$}} & 3.42 & 0.082 & 6825 & 7897 & $-15.71 \%$ & 32.33 & 9.4 \\
\hline & & & & & 0.20 & 0.004 & 258 & 654 & & & \\
\hline \multirow{3}{*}{$4 a$} & 100 & 65 & 71.18 & 3.559 & 3.56 & 0.099 & 7357 & 11211 & $-52.38 \%$ & 35.52 & \\
\hline & 100 & 62 & 67.89 & 3.395 & 3.39 & 0.094 & 7357 & 10693 & $-45.35 \%$ & 35.52 & \\
\hline & 100 & 60 & 65.70 & 3.285 & 3.29 & 0.091 & 7357 & 10348 & $-40.66 \%$ & 35.52 & \\
\hline \multirow[t]{4}{*}{$4 \mathrm{~b}$} & 100 & 57 & 62.42 & 3.121 & 3.12 & 0.090 & 7106 & 9612 & $-35.27 \%$ & 36.24 & \\
\hline & 100 & 62 & 67.89 & 3.395 & 3.39 & 0.098 & 7106 & 10456 & $-47.14 \%$ & 36.24 & \\
\hline & 100 & 63 & 68.99 & 3.449 & 3.45 & 0.099 & 7106 & 10624 & $-49.51 \%$ & 36.24 & \\
\hline & & & & & 3.37 & 0.095 & 7231 & 10491 & $-45.07 \%$ & 35.88 & 10.7 \\
\hline \multicolumn{5}{|c|}{ Recipe 4 St.Dev. } & 0.15 & 0.004 & 137 & 523 & & & \\
\hline
\end{tabular}

Notes:
1) Samples collected after 6 month composting period (Phase 2) ending November 27, 2002. 


\section{Phase 2 - Finished Compost Bulk Density Analysis}

Test Date: November 28, 2002

\begin{tabular}{|c|c|c|c|c|c|c|c|c|c|}
\hline \multirow[t]{2}{*}{ Recipe } & \multirow[t]{2}{*}{ Biocell ID } & Tare & Volume & $\begin{array}{c}\text { Mass } \\
\text { Tare+Compost }\end{array}$ & $\begin{array}{c}\text { Mass } \\
\text { Compost }\end{array}$ & $\begin{array}{c}\text { Wet Bulk } \\
\text { Density }\end{array}$ & $\begin{array}{c}\text { Moisture } \\
\text { Content }\end{array}$ & $\begin{array}{l}\text { Dry Bulk } \\
\text { Density }\end{array}$ & $\begin{array}{l}\text { Wet Bulk } \\
\text { Density }^{1}\end{array}$ \\
\hline & & $(\mathrm{kg})$ & $(L)$ & $(\mathrm{kg})$ & $(\mathrm{kg})$ & $\left(\mathrm{kg} / \mathrm{m}^{3}\right)$ & $(\%)$ & $\left(\mathrm{kg} / \mathrm{m}^{3}\right)$ & $\left(\mathrm{kg} / \mathrm{m}^{3}\right)$ \\
\hline $1 \mathrm{a}$ & 1 & 1.64 & 4.33 & 4.10 & 2.46 & 568.1 & 61.0 & 221.8 & 739.3 \\
\hline $1 b$ & 1 & 1.64 & 4.33 & 3.95 & 2.31 & 533.5 & 59.3 & 217.2 & 724.1 \\
\hline \multicolumn{8}{|c|}{ Recipe 1 Mean } & 219.5 & 731.7 \\
\hline \multicolumn{8}{|c|}{ Recipe 1 St.Dev. } & 3.2 & 10.8 \\
\hline $2 a$ & 1 & 1.64 & 4.33 & 4.27 & 2.63 & 607.4 & 50.5 & 300.8 & 1002.6 \\
\hline $2 \mathrm{~b}$ & 1 & 1.64 & 4.33 & 4.41 & 2.77 & 639.7 & 44.8 & 353.3 & 1177.7 \\
\hline \multicolumn{8}{|c|}{ Recipe 2 Mean } & 327.0 & 1090.2 \\
\hline \multicolumn{8}{|c|}{ Recipe 2 St.Dev. } & 37.2 & 123.8 \\
\hline $3 a$ & 1 & 1.64 & 4.33 & 4.55 & 2.91 & 672.1 & 45.1 & 368.8 & 1229.4 \\
\hline $3 b$ & 1 & 1.64 & 4.33 & 4.63 & 2.99 & 690.5 & 37.7 & 430.3 & 1434.2 \\
\hline \multicolumn{8}{|c|}{$\frac{1}{\text { Recipe } 3 \text { Mean }}$} & 399.5 & 1331.8 \\
\hline \multicolumn{8}{|c|}{ Recipe 3 St.Dev. } & 43.4 & 144.8 \\
\hline $4 \mathrm{a}$ & 1 & 1.64 & 4.33 & 4.14 & 2.50 & 577.4 & 58.1 & 241.7 & 805.6 \\
\hline $4 b$ & 1 & 1.64 & 4.33 & 4.09 & 2.45 & 565.8 & 62.9 & 209.7 & 699.2 \\
\hline \multicolumn{8}{|c|}{ Recipe 4 Mean } & 225.7 & 752.4 \\
\hline \multicolumn{8}{|c|}{ Recipe 4 St.Dev. } & 22.6 & 75.3 \\
\hline
\end{tabular}

Notes:

1 - Uses target moisture content of $70 \%$.

1) Small biocell (Volume $=4.33 \mathrm{~L}$ ) used for bulk density analysis. 
Phase 2 - Particle Size Analysis

Test Date: March 12, 2003

\begin{tabular}{|c|c|c|c|c|}
\hline \multirow{2}{*}{ Recipe } & Mass Tare & $\begin{array}{c}\text { Mass } \\
\text { Tare+Material }\end{array}$ & $\begin{array}{l}\text { Mass After } \\
.103^{\circ} \mathrm{C}\end{array}$ & $\begin{array}{l}\text { Moisture } \\
\text { Content }\end{array}$ \\
\hline & (g) & (g) & (g) & $(\%)$ \\
\hline 1 & 11.2 & 62.9 & 35.1 & 53.8 \\
\hline 2 & 11.2 & 65.9 & 33.5 & 59.2 \\
\hline 3 & 16.7 & 81.1 & 34.4 & 72.5 \\
\hline 4 & 11.8 & 46.4 & 23.5 & 66.2 \\
\hline
\end{tabular}

Initial Compost Recipes - Particle Size Analysis

\begin{tabular}{|c|c|c|c|c|c|c|c|c|c|c|c|}
\hline \multirow[b]{2}{*}{ Recipe } & \multirow[b]{2}{*}{$\begin{array}{c}\text { Mass of } \\
\text { Sample (g) }\end{array}$} & \multicolumn{10}{|c|}{ Mesh Size } \\
\hline & & $37.5 \mathrm{~mm}$ & $19.0 \mathrm{~mm}$ & $9.5 \mathrm{~mm}$ & $\begin{array}{c}4.8 \mathrm{~mm} \text { (Mesh } \\
\text { No. 4) }\end{array}$ & $\begin{array}{c}2.4 \mathrm{~mm} \\
\text { (Mesh No. 8) }\end{array}$ & $\begin{array}{c}1.2 \mathrm{~mm} \text { (Mesh } \\
\text { No. } 16 \text { ) }\end{array}$ & $\begin{array}{c}0.6 \mathrm{~mm} \text { (Mesh } \\
\text { No. 30) }\end{array}$ & $\begin{array}{c}0.3 \mathrm{~mm} \text { (Mesh } \\
\text { No. 50) }\end{array}$ & $\begin{array}{c}0.15 \mathrm{~mm} \\
\text { (Mesh No. 100) }\end{array}$ & Bottom Pan \\
\hline \multicolumn{12}{|c|}{ mor o 1} \\
\hline mass retained & 20.2 & 1.8 & 1.5 & 3.7 & 5.3 & 4.1 & 2.2 & 0.8 & 0.3 & 0.3 & 0.2 \\
\hline mass passing & & 18.4 & 16.9 & 13.2 & 7.9 & 3.8 & 1.6 & 0.8 & 0.5 & 0.2 & \\
\hline$\%$ passing & & 91.1 & 83.7 & 65.3 & 39.1 & 18.8 & 7.9 & 4.0 & 2.5 & 1.0 & \\
\hline \multicolumn{12}{|l|}{$\begin{array}{l}\text { \% passing } \\
\text { Recipe 2 }\end{array}$} \\
\hline mass retained & 18.5 & 0.8 & 0.9 & 2.0 & 3.4 & 4.7 & 4.5 & 1.5 & 0.3 & 0.3 & 0.1 \\
\hline mass passing & & 17.7 & 16.8 & 14.8 & 11.4 & 6.7 & 2.2 & 0.7 & 0.4 & 0.1 & \\
\hline$\%$ passing & & 95.7 & 90.8 & 80.0 & 61.6 & 36.2 & 11.9 & 3.8 & 2.2 & 0.5 & \\
\hline \multicolumn{12}{|l|}{$\begin{array}{l}\% \text { passing } \\
\text { Recipe } 3 \\
\end{array}$} \\
\hline mass retained & 17.1 & 0.0 & 0.1 & 0.6 & 4.8 & 6.3 & 3.6 & 1.3 & 0.1 & 0.2 & 0.1 \\
\hline mass passing & & 17.1 & 17.0 & 16.4 & 11.6 & 5.3 & 1.7 & 0.4 & 0.3 & 0.1 & \\
\hline$\%$ passing & & 100.0 & 99.4 & 95.9 & 67.8 & 31.0 & 9.9 & 2.3 & 1.8 & 0.6 & \\
\hline \multicolumn{12}{|l|}{ Recipe 4 } \\
\hline mass retained & 11.8 & 0.0 & 0.0 & 0.1 & 1.9 & 4.9 & 3.1 & 1.0 & 0.5 & 0.2 & 0.1 \\
\hline mass passing & & 11.8 & 11.8 & 11.7 & 9.8 & 4.9 & 1.8 & 0.8 & 0.3 & 0.1 & \\
\hline$\%$ passing & & 100.0 & 100.0 & 99.2 & 83.1 & 41.5 & 15.3 & 6.8 & 2.5 & 0.8 & \\
\hline
\end{tabular}

Notes:

1) Particle size tests completed according to ASTM Standard Method D422 - 93 (1990).

Initial Compost Recipes - Coefficlents of Uniformity

$$
\text { Coefficient of Uniformity: } C_{U}=D_{60} / D_{10} \quad \text { - higher } C u \text { indicates larger range of particle sizes. }
$$

Coefficient of Curvature: $C_{Z}=D^{2}{ }_{30} / D_{60} D_{10} \quad$ - a well graded material has a $C z$ bewteen 1 and 3 .

\begin{tabular}{|c|c|c|c|c|c|}
\hline Recipe & $D_{10}$ & $D_{30}$ & $D_{60}$ & $C_{U}$ & $C_{z}$ \\
\hline 1 & 1.5 & 3.6 & 8.0 & 5.3 & 1.1 \\
\hline 2 & 1.1 & 2.0 & 4.7 & 4.3 & 0.8 \\
\hline 3 & 1.1 & 2.3 & 4.1 & 3.7 & 1.2 \\
\hline 4 & 0.9 & 1.8 & 3.2 & 3.6 & 1.1 \\
\hline
\end{tabular}


Phase 2 - Particle Size Analysis

Test Date: March 12, 2003

Finished Compost Recipes - Particle Size Analysis

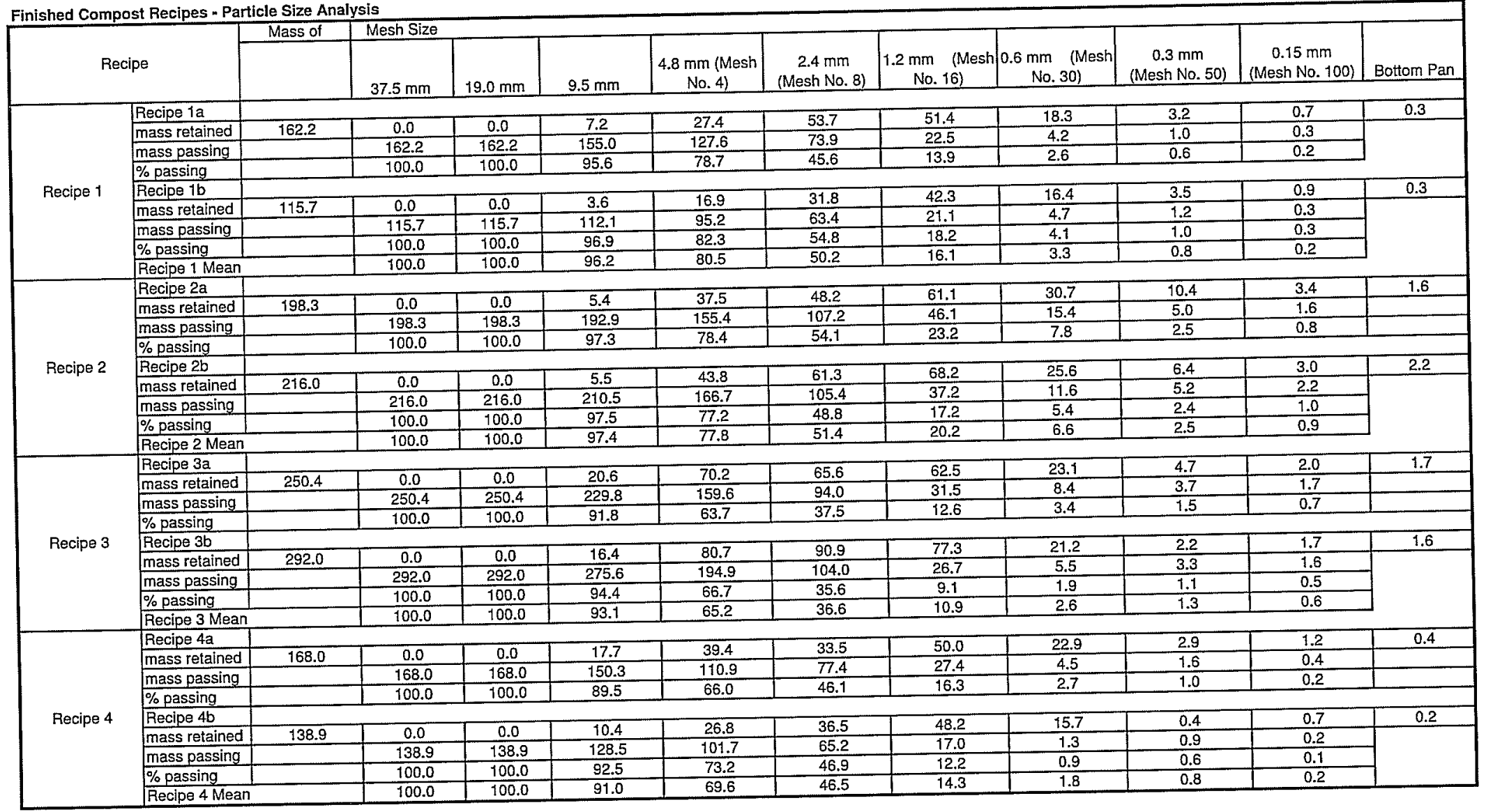

Notes:

1) Particle size tests completed according to ASTM Standard Method D422 - 93 (1990). 


\section{Phase 2 - Particle Size Analysis}

Test Date: March 12, 2003

Finished Compost Recipes - Coefficients of Uniformity

Coefficient of Uniformity: $C_{U}=D_{60} / D_{10}$

- higher $\mathrm{Cu}$ indicates larger range of particle sizes.

Coeficient of Curvature: $C_{z}=D^{2}{ }^{2} / D_{60} D_{10}$

- a well graded material has a $\mathrm{C} z$ bewteen 1 and 3 .

\begin{tabular}{|c|c|c|c|c|c|c|}
\hline Recipe & Reactor & $\mathrm{D}_{10}$ & $\mathrm{D}_{30}$ & $\mathrm{D}_{60}$ & $\mathrm{C}_{\mathrm{U}}$ & $\mathrm{C}_{\mathrm{z}}$ \\
\hline 1 & $1 \mathrm{a}$ & 1.0 & 1.7 & 3.3 & 3.3 & 0.9 \\
\hline & $1 \mathrm{~b}$ & 0.9 & 1.5 & 2.7 & 3.0 & 0.9 \\
\hline Mean $=$ & & 1.0 & 1.6 & 3.0 & 3.2 & 0.9 \\
\hline 2 & $2 \mathrm{a}$ & 0.7 & 1.4 & 2.8 & 4.0 & 1.0 \\
\hline & $2 \mathrm{~b}$ & 0.8 & 1.5 & 3.2 & 4.0 & 0.9 \\
\hline Mean $=$ & & 0.8 & 1.5 & 3.0 & 4.0 & 0.9 \\
\hline 3 & $3 \mathrm{a}$ & 1.0 & 2.0 & 4.4 & 4.4 & 0.9 \\
\hline Mean = & $3 \mathrm{~b}$ & 1.2 & 2.1 & 4.1 & 3.4 & 0.9 \\
\hline 4 & & 1.1 & 2.1 & 4.3 & 3.9 & 0.9 \\
\hline & $4 \mathrm{a}$ & 1.0 & 1.7 & 3.9 & 3.9 & 0.7 \\
\hline Mean = & $4 \mathrm{~b}$ & 1.0 & 1.7 & 3.3 & 3.3 & 0.9 \\
\hline & & 1.0 & 1.7 & 3.6 & 3.6 & 0.8 \\
\hline
\end{tabular}




\section{Phase 2 - Finished Compost Particle Density Analysis}

Test Date: February 13-15, 2003

$$
\begin{aligned}
\rho_{\mathrm{p}} & \neq \text { article density }\left(\mathrm{g} / \mathrm{cm}^{3}\right) \\
\rho_{\mathrm{w}} & \text { } \\
\mathrm{W}_{\mathrm{s}} & =\text { wensity of water }\left(1 \mathrm{~g} / \mathrm{cm}^{3}\right) \\
\mathrm{W}_{\mathrm{a}} & =\text { weight of pyconometer plus sample }(\mathrm{g}) \\
\mathrm{W}_{\mathrm{sw}} & =\rho_{\mathrm{w}}\left(\mathrm{W}_{\mathrm{s}}-\mathrm{W}_{\mathrm{a}}\right) /\left[\left(\mathrm{W}_{\mathrm{s}}-\mathrm{W}_{\mathrm{a}}\right)-\left(\mathrm{W}_{\mathrm{sw}}-\mathrm{W}_{\mathrm{w}}\right)\right] \\
\mathrm{W}_{\mathrm{w}} & =\text { weight of pyconometer filled with air }(\mathrm{g})
\end{aligned}
$$

\begin{tabular}{|c|c|c|c|c|c|c|c|}
\hline \multirow{2}{*}{ Recipe } & \multirow{2}{*}{ Flask } & $\mathrm{W}_{\mathrm{a}}$ & $W_{w}$ & $W_{s}$ & $W_{\text {sw }}$ & $\rho_{w}$ & $\rho_{p}$ \\
\hline & & (g) & (g) & (g) & (g) & $\left(\mathrm{g} / \mathrm{cm}^{3}\right)$ & $\left(\mathrm{g} / \mathrm{cm}^{3}\right)$ \\
\hline $1 \mathrm{a}$ & 2 & 242.1 & $\frac{191}{1227.0}$ & 292.1 & 1234.6 & 1 & 1.18 \\
\hline$\frac{m}{1 a}$ & 3 & 234.3 & 1217.1 & 284.36 & 1225.6 & 1 & 1.20 \\
\hline$\frac{a b}{1 b}$ & $\frac{u}{E}$ & 272.8 & 1253.1 & 322.8 & 1261.6 & $\frac{1}{1}$ & 1.20 \\
\hline $1 \mathrm{~b}$ & $\mathrm{C}$ & 220.5 & 1214.0 & 270.5 & 1223.2 & 1 & 1.23 \\
\hline \multicolumn{7}{|c|}{ Recipe 1 Mean } & 1.20 \\
\hline \multicolumn{7}{|c|}{ Recipe 1 St. Dev. } & 0.02 \\
\hline $2 a$ & ED & 226.2 & 1218.1 & 276.2 & 1226.4 & 1 & 1.20 \\
\hline$\frac{2 a}{2 a}$ & $B$ & 226.1 & 1218.7 & 276.1 & 1229.2 & 1 & 1.27 \\
\hline $2 b$ & $\mathrm{E}$ & 272.8 & 1253.1 & 322.8 & 1266.4 & 1 & 1.36 \\
\hline $2 \mathrm{~b}$ & $\mathrm{C}$ & 220.5 & 1214.0 & 270.5 & 1225.7 & 1 & 1.31 \\
\hline \multicolumn{7}{|c|}{ Recipe 2 Mean } & 1.28 \\
\hline \multicolumn{7}{|c|}{ Recipe 2 St. Dev. } & 0.07 \\
\hline $3 a$ & $\mathrm{~B}$ & 226.1 & 1218.7 & 276.1 & 1227.8 & 1 & 1.22 \\
\hline $3 a$ & $E$ & 272.8 & 1253.1 & 322.8 & 1262.7 & 1 & 1.24 \\
\hline $3 \mathrm{~b}$ & 2 & 242.1 & 1227.0 & 292.1 & 1240.1 & 1 & 1.36 \\
\hline $3 b$ & 3 & 234.3 & 1217.1 & 284.3 & 1229.4 & 1 & 1.33 \\
\hline \multicolumn{7}{|c|}{ Recipe 3 Mean } & 1.29 \\
\hline \multicolumn{7}{|c|}{ Recipe 3 St. Dev. } & 0.07 \\
\hline $4 a$ & ED & 226.2 & 1218.1 & 276.2 & 1223.1 & 1 & 1.11 \\
\hline$\frac{4 a}{4 a}$ & $E$ & 272.8 & 1253.1 & 322.8 & 1256.9 & $\frac{1}{1}$ & 1.08 \\
\hline $4 b$ & 2 & 242.1 & 1227.0 & 292.1 & 1233.0 & 1 & 1.14 \\
\hline $4 \mathrm{~b}$ & $B$ & 226.1 & 1218.7 & 276.1 & 1223.7 & 1 & 1.11 \\
\hline \multicolumn{7}{|c|}{ Recipe 4 Mean } & 1.11 \\
\hline \multicolumn{7}{|c|}{ Recipe 4 St. Dev. } & 0.02 \\
\hline
\end{tabular}

Notes:

1) Particle density tests performed with $50 \mathrm{~g}$ compost samples.

2) Sample drying was completed in a $103^{\circ} \mathrm{C}$ oven for 24 hours.

3) Room temperature for particle density test was $21.0^{\circ} \mathrm{C}$. 
Phase 2 - Statistical Analysis of Volatile Solids Analysis (Day 180)

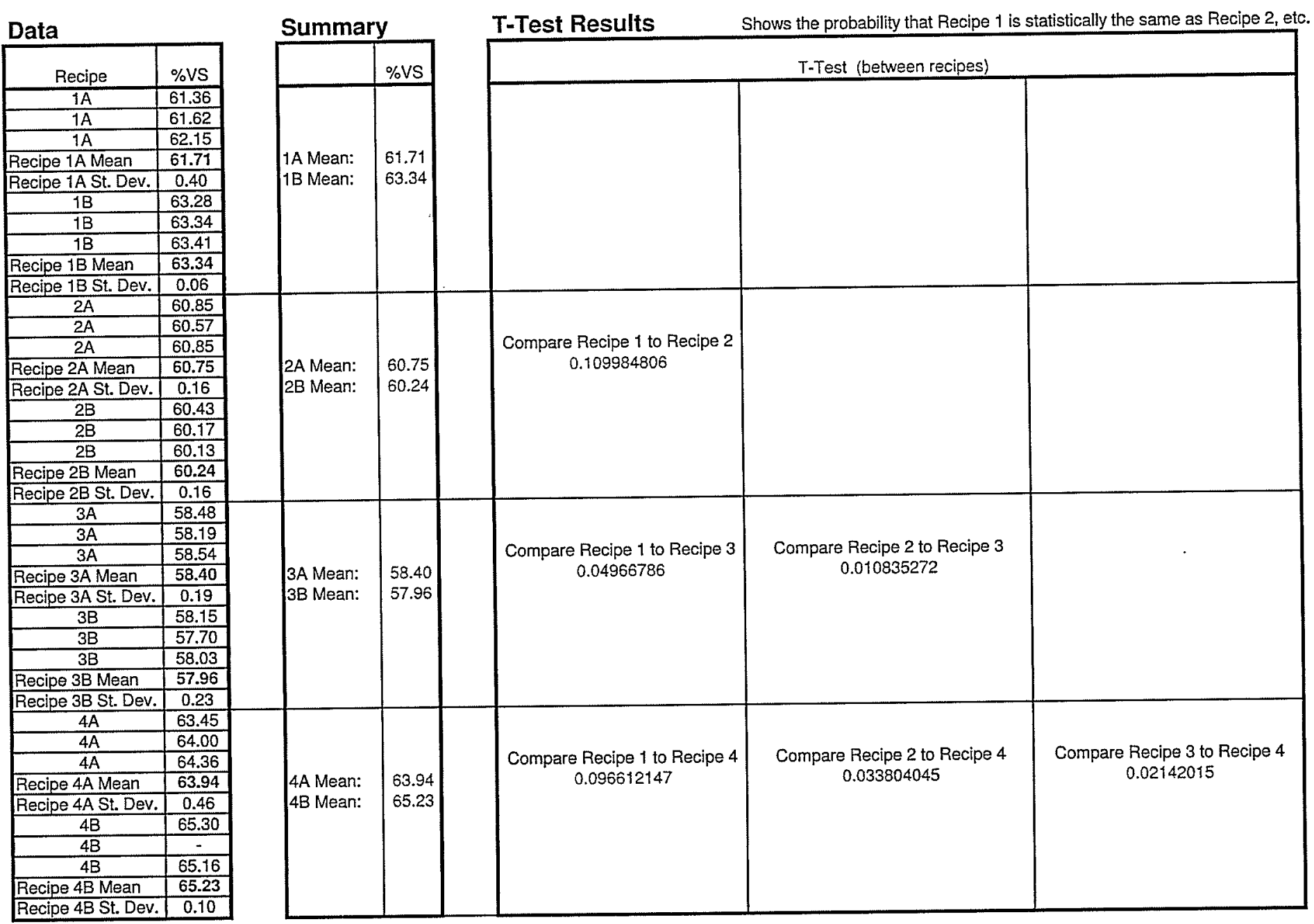


Phase 2 - Statistical Analysis of g Lignin / g Ash (Day 180)

\begin{tabular}{|c|c|c|c|c|}
\hline Reactor & g Lignin/gAsh & T-test Results (shows the $\mathrm{p}$ & bability that Recipe 1 is statistical & the same as Reci \\
\hline $1 \mathrm{a}$ & 0.5048 & & & \\
\hline $1 \mathrm{~b}$ & 0.5217 & & & \\
\hline $\begin{array}{l}\text { Recipe } 1 \text { Mean } \\
\text { Recipe 1 St.Dev. }\end{array}$ & $\begin{array}{r}0.5132 \\
0.0120 \\
\end{array}$ & & & \\
\hline 2a & 0.4273 & & & \\
\hline \begin{tabular}{|l|}
$\mathrm{b} \mathrm{b}$ \\
Recipe 2Mean
\end{tabular} & 0.4330 & Compare Recipe 1 to Recipe 2 & & \\
\hline $\begin{array}{l}\text { Recipe } 2 \text { Mean } \\
\text { Recipe } 2 \text { St.Dev. }\end{array}$ & 0.4302 & 0.021831446 & & \\
\hline Heclpe 2 st.vev. & $\frac{0.0040}{0.4029}$ & & & \\
\hline $3 \mathrm{~b}$ & 0.4003 & Compare Recipe 1 to Recipe 3 & Compare Recipe 2 to Recipe 3 & \\
\hline Recipe 3 Mean & 0.4016 & 0.021784056 & & \\
\hline $\begin{array}{l}\text { Recipe 3 St.Dev. } \\
\text { 4a }\end{array}$ & $\begin{array}{r}0.0018 \\
0.5792 \\
\end{array}$ & & & \\
\hline $4 \mathrm{~b}$ & 0.6264 & Compare Recipe 1 to Recipe 4 & Compare Recipe 2 to Recipe 4 & $\begin{array}{c}\text { Compare Recipe } 3 \text { to Recipe } 4 \\
0.036787673\end{array}$ \\
\hline \begin{tabular}{|l} 
Recipe 4 Mean \\
Recipe 4 St.Dev.
\end{tabular} & $\frac{0.6028}{0.0334}$ & & & \\
\hline
\end{tabular}


Phase 2 - Statistical Analysis of $g$ Cellulose / g Ash (Day 180)

\begin{tabular}{|c|c|c|c|c|}
\hline Reactor & gGellulose /g Ash & \multicolumn{3}{|c|}{ T-test Results (shows the probability that Recipe 1 is statistically the same as Recipe 2 , etc.) } \\
\hline $1 \mathrm{a}$ & 0.8029 & & & \\
\hline $1 \mathrm{~b}$ & 0.7947 & & & \\
\hline \begin{tabular}{|l} 
Recipe 1 Mean \\
Recipe I St.Dev.
\end{tabular} & 0.7988 & & & \\
\hline $\begin{array}{l}\text { Recipe } 1 \text { St.Dev. } \\
2 a\end{array}$ & $\begin{array}{l}0.0058 \\
0.7639 \\
\end{array}$ & \multirow{4}{*}{$\begin{array}{c}\text { Compare Recipe } 1 \text { to Recipe } 2 \\
0.072942785\end{array}$} & & \\
\hline$\frac{2 a}{2 b}$ & 0.7639 & & & \\
\hline Recipe 2 Mean & 0.7507 & & & \\
\hline$\frac{\text { Recipe 2 St.Dev. }}{\text { 3a }}$ & 0.0187 & & \multirow{3}{*}{$\begin{array}{c}\text { Compare Recipe } 2 \text { to Recipe } 3 \\
0.297413632\end{array}$} & \\
\hline $3 a$ & $\begin{array}{ll}0.7201 \\
0.7541 \\
\end{array}$ & \multirow{3}{*}{$\begin{array}{c}\text { Compare Recipe } 1 \text { to Recipe } 3 \\
0.077523594\end{array}$} & & \\
\hline Recipe 3 Mean & 0.7371 & & & \\
\hline Recipe 3 St.Dev. & 0.0241 & & \multirow{4}{*}{$\begin{array}{c}\text { Compare Recipe } 2 \text { to Recipe } 4 \\
0.023215271\end{array}$} & \multirow{4}{*}{$\begin{array}{c}\text { Compare Recipe } 3 \text { to Recipe } 4 \\
0.031775294\end{array}$} \\
\hline$\frac{4 a}{4 b}$ & 0.8223 & \multirow{3}{*}{$\begin{array}{c}\text { Compare Recipe } 1 \text { to Recipe } 4 \\
0.067990119\end{array}$} & & \\
\hline Recipe 4 Mean & 0.8411 & & & \\
\hline Recipe 4 St.Dev. & 0.0133 & & & \\
\hline
\end{tabular}


Phase 2 - Statistical Analysis of g Hemicellulose / g Ash (Day 180)

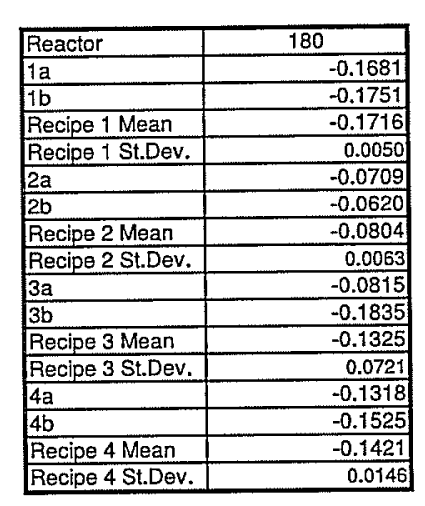

No Statistical Analysis Performed on Hemicellulose Data (All Values $<0$ )

岕 


\section{Phase 2 - Hemicellulose Determination}

Technician: Janice Haines/Scott Chapman

Date: February 5, 2003

\begin{tabular}{|c|c|c|c|c|c|}
\hline $\begin{array}{l}\text { Samp } \\
\text { ID }\end{array}$ & $\begin{array}{l}\text { Day } \\
\text { No. }\end{array}$ & Date & $\begin{array}{c}\% \text { NDF } \\
\text { DM Basis }\end{array}$ & $\begin{array}{c}\% \text { ADF } \\
\text { DM Basis }\end{array}$ & $\begin{array}{c}\text { Hemicellulose } \\
\% \mathrm{DM}\end{array}$ \\
\hline $1 \mathrm{~A}$ & 18 & June $18 / 02$ & 73.6682395 & 60.5729267 & 13.09531287 \\
\hline $1 \mathrm{~B}$ & 18 & June $18 / 02$ & 74.207179 & 56.8146216 & 17.39255741 \\
\hline $2 \mathrm{~A}$ & 18 & June $18 / 02$ & 68.0195347 & 66.7912189 & 1.228315797 \\
\hline $2 \mathrm{~B}$ & 18 & June $18 / 02$ & 57.8378754 & 48.8176144 & 9.020260975 \\
\hline $3 \mathrm{~A}$ & 18 & June 18/02 & 62.6861118 & 47.267651 & 15.41846084 \\
\hline $3 B$ & 18 & June $18 / 02$ & 60.5043816 & 48.2128609 & 12.29152072 \\
\hline $4 \mathrm{~A}$ & 18 & June $18 / 02$ & 62.129488 & 46.9600137 & 15.16947424 \\
\hline $4 \mathrm{~B}$ & 18 & June $18 / 02$ & 59.0590944 & 48.3375284 & 10.72156606 \\
\hline$\overline{1 \mathrm{~A}}$ & 46 & July $16 / 02$ & 62.3106268 & 59.5147997 & 2.79582712 \\
\hline $1 \mathrm{~B}$ & 46 & July $16 / 02$ & 63.9670368 & 52.7459473 & 11.22108952 \\
\hline $2 \mathrm{~A}$ & 46 & July $16 / 02$ & 52.6809759 & 42.4439476 & 10.23702833 \\
\hline $2 \mathrm{~B}$ & 46 & July $16 / 02$ & 46.8464526 & 42.3350756 & 4.511377009 \\
\hline $3 \mathrm{~A}$ & 46 & July $16 / 02$ & 52.2611139 & 42.70842 & 9.55269386 \\
\hline $3 B$ & 46 & July $16 / 02$ & 42.2934408 & 34.1511343 & 8.142306498 \\
\hline $4 \mathrm{~A}$ & 46 & July $16 / 02$ & 50.8597693 & 42.8644687 & 7.995300611 \\
\hline $4 \mathrm{~B}$ & 46 & July $16 / 02$ & 53.2820903 & 44.994591 & 8.287499287 \\
\hline $1 \mathrm{~A}$ & 74 & Aug 13/02 & 60.204494 & 47.7294169 & 12.47507712 \\
\hline $1 \mathrm{~B}$ & 74 & Aug 13/02 & 58.2248728 & 54.0248166 & 4.20005624 \\
\hline $2 \mathrm{~A}$ & 74 & Aug 13/02 & 48.1384008 & 42.5332785 & 5.605122277 \\
\hline $2 \mathrm{~B}$ & 74 & Aug 13/02 & 43.7444568 & 41.3759192 & 2.368537567 \\
\hline $3 \mathrm{~A}$ & 74 & Aug 13/02 & 38.8983266 & 39.9101942 & -1.011867595 \\
\hline $3 \mathrm{~B}$ & 74 & Aug 13/02 & 41.0102287 & 40.5732688 & 0.436959863 \\
\hline $4 \mathrm{~A}$ & 74 & Aug 13/02 & 51.8099703 & 55.5022182 & -3.692247963 \\
\hline $4 \mathrm{~B}$ & 74 & Aug 13/02 & 55.4446895 & 53.3658461 & 2.078843333 \\
\hline $1 \mathrm{~A}$ & 109 & Sept $17 / 02$ & 42.4307856 & 44.8487471 & -2.417961471 \\
\hline $1 \mathrm{~B}$ & 109 & Sept $17 / 02$ & 51.5813808 & 50.3129232 & 1.268457593 \\
\hline $2 \mathrm{~A}$ & 109 & Sept $17 / 02$ & 38.3723822 & 42.5831626 & -4.210780402 \\
\hline $2 B$ & 109 & Sept $17 / 02$ & 35.358529 & 45.9719659 & -10.61343687 \\
\hline $3 \mathrm{~A}$ & 109 & Sept $17 / 02$ & 41.8589152 & 49.8952338 & -8.036318612 \\
\hline $3 B$ & 109 & Sept $17 / 02$ & 39.3868236 & 40.991613 & -1.604789339 \\
\hline $4 \mathrm{~A}$ & 109 & Sept 17/02 & 48.6141579 & 51.7903077 & -3.176149806 \\
\hline $4 \mathrm{~B}$ & 109 & Sept 17/02 & 54.4912941 & 50.172728 & 4.318566044 \\
\hline
\end{tabular}




\section{Phase 2 - Hemicellulose Determination}

Technician: Janice Haines/Scott Chapman

Date: February 5, 2003

\begin{tabular}{|c|c|c|c|c|c|}
\hline $\begin{array}{c}\text { Samp } \\
\text { ID }\end{array}$ & $\begin{array}{l}\text { Day } \\
\text { No. }\end{array}$ & Date & $\begin{array}{c}\% \text { NDF } \\
\text { DM Basis }\end{array}$ & $\begin{array}{c}\% \text { ADF } \\
\text { DM Basis }\end{array}$ & $\begin{array}{c}\text { Hemicellulose } \\
\% D M\end{array}$ \\
\hline $1 \mathrm{~A}$ & 123 & Oct $1 / 02$ & 46.4543462 & 49.9198144 & -3.465468194 \\
\hline $1 \mathrm{~B}$ & 123 & Oct $1 / 02$ & 51.6638179 & 45.893709 & 5.770108845 \\
\hline $2 \mathrm{~A}$ & 123 & Oct $1 / 02$ & 46.7957693 & 40.7722034 & 6.02356586 \\
\hline $2 \mathrm{~B}$ & 123 & Oct $1 / 02$ & 45.7266334 & 42.7597598 & 2.966873625 \\
\hline $3 A$ & 123 & Oct $1 / 02$ & 43.9664454 & 44.8714067 & -0.904961291 \\
\hline $3 \mathrm{~B}$ & 123 & Oct $1 / 02$ & 38.7613771 & 49.0401689 & -10.27879176 \\
\hline $4 \mathrm{~A}$ & 123 & Oct $1 / 02$ & 46.5797297 & 51.0429786 & -4.463248933 \\
\hline $4 \mathrm{~B}$ & 123 & Oct $1 / 02$ & 53.0438765 & 54.4591877 & -1.415311257 \\
\hline $1 \mathrm{~A}$ & 137 & Oct $15 / 02$ & 39.664833 & 41.7686499 & -2.10381693 \\
\hline $1 \mathrm{~B}$ & 137 & Oct $15 / 02$ & 40.9936127 & 47.4504626 & -6.456849959 \\
\hline $2 \mathrm{~A}$ & 137 & Oct $15 / 02$ & 47.6396867 & 46.2699444 & 1.3697423 \\
\hline $2 \mathrm{~B}$ & 137 & Oct $15 / 02$ & 45.5824026 & 47.144973 & -1.56257043 \\
\hline $3 A$ & 137 & Oct $15 / 02$ & 47.1699905 & 43.7050056 & 3.46498489 \\
\hline $3 B$ & 137 & Oct 15/02 & 41.2865047 & 40.7483964 & 0.538108273 \\
\hline $4 \mathrm{~A}$ & 137 & Oct $15 / 02$ & 49.2037788 & 45.8185234 & 3.385255343 \\
\hline $4 \mathrm{~B}$ & 137 & Oct $15 / 02$ & 51.8830956 & 45.5368521 & 6.346243564 \\
\hline $1 \mathrm{~A}$ & 158 & Nov $5 / 02$ & 37.8090235 & 51.005087 & -13.19606351 \\
\hline $1 \mathrm{~B}$ & 158 & Nov $5 / 02$ & 42.0024328 & 48.4474608 & -6.445028024 \\
\hline $2 A$ & 158 & Nov $5 / 02$ & 36.7968872 & 45.3208169 & -8.523929719 \\
\hline $2 \mathrm{~B}$ & 158 & Nov $5 / 02$ & 40.3688389 & 45.5217013 & -5.15286242 \\
\hline $3 A$ & 158 & Nov $5 / 02$ & 40.481603 & 48.0625514 & -7.580948442 \\
\hline $3 \mathrm{~B}$ & 158 & Nov $5 / 02$ & 36.7985718 & 41.11426 & -4.315688202 \\
\hline $4 \mathrm{~A}$ & 158 & Nov $5 / 02$ & 45.9284304 & 49.5011056 & -3.572675177 \\
\hline $4 \mathrm{~B}$ & 158 & Nov $5 / 02$ & 47.1734171 & 48.2879188 & -1.114501689 \\
\hline $1 \mathrm{~A}$ & 180 & Nov $27 / 02$ & 43.6319303 & 50.0671577 & -6.435227454 \\
\hline $1 \mathrm{~B}$ & 180 & Nov $27 / 02$ & 41.8345106 & 48.2540268 & -6.419516143 \\
\hline $2 \mathrm{~A}$ & 180 & Nov $27 / 02$ & 43.9679687 & 46.7499945 & -2.782025854 \\
\hline $2 \mathrm{~B}$ & 180 & Nov 27/02 & 44.0693737 & 46.5340065 & -2.464632768 \\
\hline $3 \mathrm{~A}$ & 180 & Nov $27 / 02$ & 43.3182762 & 46.7094979 & -3.391221747 \\
\hline $3 \mathrm{~B}$ & 180 & Nov $27 / 02$ & 40.8134593 & 48.5253717 & -7.711912349 \\
\hline$\overline{4 A}$ & 180 & Nov $27 / 02$ & 45.7875616 & 50.5401608 & -4.752599186 \\
\hline $4 \mathrm{~B}$ & 180 & Nov $27 / 02$ & 45.7239865 & 51.0268757 & -5.302889172 \\
\hline KWSTD & & & 36.9425157 & 29.5068445 & 7.435671205 \\
\hline
\end{tabular}


Phase 2 - Cellulose Determination

Technician: Janice Haines/Scott Chapman

Date: February 5, 2002

\begin{tabular}{|c|c|c|c|c|c|}
\hline $\begin{array}{l}\text { Samp } \\
\text { ID }\end{array}$ & $\begin{array}{l}\text { Day } \\
\text { No. }\end{array}$ & Date & $\begin{array}{l}\% \text { ADF } \\
\text { DM Basis }\end{array}$ & $\mid \begin{array}{l}\% \text { Lignin } \\
\text { DM Basis }\end{array}$ & $\begin{array}{l}\text { \% Cellulose } \\
\text { DM Basis }\end{array}$ \\
\hline $1 \mathrm{~A}$ & 18 & June $18 / 02$ & 60.57292666 & 15.6958286 & 44.87709803 \\
\hline $1 \mathrm{~B}$ & 18 & June $18 / 02$ & 56.81462163 & 13.2472323 & 43.56738932 \\
\hline $2 \mathrm{~A}$ & 18 & June $18 / 02$ & 66.79121885 & 17.6170369 & 49.17418193 \\
\hline $2 \mathrm{~B}$ & 18 & June $18 / 02$ & 48.81761443 & 13.1455359 & 35.67207854 \\
\hline $3 A$ & 18 & June 18/02 & 47.26765098 & 14.1692018 & 33.09844917 \\
\hline $3 \mathrm{~B}$ & 18 & June $18 / 02$ & 48.21286091 & 14.0835151 & 34.12934578 \\
\hline $4 \mathrm{~A}$ & 18 & June $18 / 02$ & 46.96001374 & 14.3527733 & 32.60724047 \\
\hline $4 \mathrm{~B}$ & 18 & June $18 / 02$ & 48.33752837 & 14.3345004 & 34.00302797 \\
\hline $1 \mathrm{~A}$ & 46 & \begin{tabular}{|l} 
July $16 / 02$ \\
\end{tabular} & 59.51479971 & 16.5053494 & 43.00945035 \\
\hline $1 \mathrm{~B}$ & 46 & \begin{tabular}{|l} 
July $16 / 02$ \\
\end{tabular} & 52.74594729 & 15.1915793 & 37.55436803 \\
\hline $2 A$ & 46 & July $16 / 02$ & 42.4439476 & 14.1040266 & 28.33992102 \\
\hline $2 \mathrm{~B}$ & 46 & July $16 / 02$ & 42.33507557 & 15.4709513 & 26.86412426 \\
\hline $3 \mathrm{~A}$ & 46 & July $16 /$ & 42.70841999 & 15.0184953 & 27.68992467 \\
\hline $3 \mathrm{~B}$ & 46 & July $16 /$ & 34.15113426 & 13.00991 & 21.14122424 \\
\hline $4 \mathrm{~A}$ & 46 & July $16 / 02$ & 42.86446867 & 15.2737355 & 27.59073317 \\
\hline $4 \mathrm{~B}$ & 46 & July $16 / 02$ & 44.99459101 & 15.8609659 & 29.13362515 \\
\hline $1 \mathrm{~A}$ & 74 & Aug 13/02 & 47.72941691 & 17.1931615 & 30.53625545 \\
\hline $1 \mathrm{~B}$ & 74 & Aug 13/02 & 54.02481657 & 17.6035751 & 36.42124147 \\
\hline $2 \mathrm{~A}$ & 74 & Aug 13/02 & 42.5332785 & 14.7487011 & 27.78457736 \\
\hline $2 \mathrm{~B}$ & 74 & Aug 13/02 & 41.37591925 & 17.0398724 & 24.33604689 \\
\hline $3 \mathrm{~A}$ & 74 & Aug 13/02 & 39.91019417 & 15.1296409 & 24.78055325 \\
\hline $3 \mathrm{~B}$ & 74 & Aug 13/02 & 40.57326881 & 15.7846916 & 24.78857724 \\
\hline $4 \mathrm{~A}$ & 74 & Aug 13/02 & 55.50221825 & 22.0074409 & 33.49477731 \\
\hline $4 \mathrm{~B}$ & 74 & Aug 13/02 & 53.36584613 & 20.6992819 & 32.66656422 \\
\hline $1 \mathrm{~A}$ & 109 & Sept $17 / 02$ & 44.84874707 & 17.1289817 & 27.71976535 \\
\hline 1B & 109 & Sept $17 / 02$ & 50.31292323 & 16.3861253 & 33.9267979 \\
\hline $2 \mathrm{~A}$ & 109 & Sept 17/02 & 42.58316261 & 16.0575546 & 26.52560803 \\
\hline $2 \mathrm{~B}$ & 109 & Sept 17/02 & 45.9719659 & 16.8761452 & 29.09582068 \\
\hline $3 \mathrm{~A}$ & 109 & Sept $17 / 02$ & 49.8952338 & 17.6521352 & 32.2430986 \\
\hline $3 \mathrm{~B}$ & 109 & Sept 17/02 & 40.99161296 & 15.6229836 & 25.36862935 \\
\hline $4 \mathrm{~A}$ & 109 & Sept $17 / 02$ & 51.79030773 & 21.2619226 & 30.5283851 \\
\hline $4 \mathrm{~B}$ & 109 & Sept 17/02 & 50.17272804 & 21.2028648 & 28.96986325 \\
\hline
\end{tabular}




\section{Phase 2 - Cellulose Determination}

Technician: Janice Haines/Scott Chapman

Date: February 5, 2002

\begin{tabular}{|c|c|c|c|c|c|}
\hline $\begin{array}{l}\text { Samp } \\
\text { ID }\end{array}$ & $\begin{array}{l}\text { Day } \\
\text { No. }\end{array}$ & Date & $\begin{array}{l}\% \text { ADF } \\
\text { DM Basis }\end{array}$ & $\begin{array}{l}\% \text { Lignin } \\
\text { DM Basis }\end{array}$ & $\begin{array}{l}\text { \% Cellulose } \\
\text { DM Basis }\end{array}$ \\
\hline $1 \mathrm{~A}$ & 123 & Oct $1 / 02$ & 49.91981442 & 18.535703 & 31.38411145 \\
\hline $1 \mathrm{~B}$ & 123 & Oct $1 / 02$ & 45.89370901 & 16.6896889 & 29.20402016 \\
\hline $2 \mathrm{~A}$ & 123 & Oct $1 / 02$ & 40.77220345 & 16.1798691 & 24.59233436 \\
\hline $2 \mathrm{~B}$ & 123 & Oct $1 / 02$ & 42.75975977 & 15.1818535 & \begin{tabular}{|l|}
27.57790627 \\
\end{tabular} \\
\hline $3 \mathrm{~A}$ & 123 & Oct $1 / 02$ & 44.87140673 & 16.2850414 & 28.58636532 \\
\hline $3 \mathrm{~B}$ & 123 & Oct $1 / 02$ & 49.04016889 & 16.9073296 & 32.13283933 \\
\hline $4 \mathrm{~A}$ & 123 & Oct $1 / 02$ & 51.04297861 & 21.9262994 & 29.11667918 \\
\hline $4 \mathrm{~B}$ & 123 & Oct $1 / 02$ & 54.45918772 & 23.881458 & 30.57772972 \\
\hline $1 \mathrm{~A}$ & 137 & Oct 15/02 & 41.76864989 & 16.5813886 & 25.18726127 \\
\hline 1B & 137 & Oct $15 / 02$ & 47.45046265 & 17.9669217 & 29.48354098 \\
\hline $2 A$ & 137 & Oct 15/02 & 46.26994438 & 17.1941194 & 29.07582498 \\
\hline $2 \mathrm{~B}$ & 137 & Oct $15 / 02$ & 47.144973 & 16.3894889 & 30.75548408 \\
\hline $3 A$ & 137 & Oct $15 / 02$ & 43.70500565 & 15.5136974 & 28.19130821 \\
\hline $3 \mathrm{~B}$ & 137 & Oct $15 / 02$ & 40.7483964 & 14.1476765 & 26.60071994 \\
\hline $4 \mathrm{~A}$ & 137 & Oct $15 / 02$ & 45.81852343 & 19.452041 & 26.36648244 \\
\hline $4 \mathrm{~B}$ & 137 & Oct $15 / 02$ & 45.53685206 & 20.549303 & 24.98754909 \\
\hline $1 \mathrm{~A}$ & 158 & Nov $5 / 02$ & 51.00508704 & 18.1157589 & 32.8893281 \\
\hline $1 \mathrm{~B}$ & 158 & Nov 5/02 & 48.44746082 & 17.9357852 & 30.51167561 \\
\hline $2 \mathrm{~A}$ & 158 & Nov $5 / 02$ & 45.32081694 & 15.4811377 & 29.83967919 \\
\hline $2 B$ & 158 & Nov $5 / 02$ & 45.52170129 & 15.8877805 & 29.63392079 \\
\hline$\overline{3 A}$ & 158 & Nov $5 / 02$ & 48.0625514 & 15.9620145 & 32.10053686 \\
\hline $3 \mathrm{~B}$ & 158 & Nov $5 / 02$ & 41.11426003 & 14.2056982 & 26.90856181 \\
\hline $4 \mathrm{~A}$ & 158 & Nov 5/02 & 49.50110558 & 19.8066787 & 29.69442692 \\
\hline $4 \mathrm{~B}$ & 158 & Nov $5 / 02$ & 48.2879188 & 19.1910191 & 29.09689973 \\
\hline $1 \mathrm{~A}$ & 180 & Nov $27 / 02$ & 50.06715774 & 19.3266388 & 30.74051892 \\
\hline 1B & 180 & Nov $27 / 02$ & 48.25402679 & 19.1239849 & 29.13004194 \\
\hline $2 \mathrm{~A}$ & 180 & Nov $27 / 02$ & 46.74999451 & 16.7692412 & 29.9807533 \\
\hline $2 \mathrm{~B}$ & 180 & Nov $27 / 02$ & 46.53400648 & 17.2145438 & 29.31946273 \\
\hline $3 \mathrm{~A}$ & 180 & Nov $27 / 02$ & 46.70949794 & 16.7574435 & 29.95205439 \\
\hline $3 \mathrm{~B}$ & 180 & Nov 27/02 & 48.52537169 & 16.8257445 & 31.69962721 \\
\hline $4 \mathrm{~A}$ & 180 & Nov $27 / 02$ & 50.54016081 & 20.8878436 & 29.65231724 \\
\hline $4 \mathrm{~B}$ & 180 & Nov $27 / 02$ & 51.02687568 & 21.7822076 & 29.24466806 \\
\hline KWSTD & & & 29.50684446 & 5.05205799 & 24.45478647 \\
\hline
\end{tabular}


Phase 2 - L.Ignin DetermInation

Technician: Janice Haines

Date: February 5, 2003

Blank: 1.00034545

\begin{tabular}{|c|c|c|c|c|c|c|c|c|c|c|}
\hline $\begin{array}{c}\text { Sample } \\
\text { ID }\end{array}$ & $\begin{array}{l}\text { Day } \\
\text { No. }\end{array}$ & Sample Data & $\begin{array}{c}\text { Sample Dry } \\
\text { Weight }\end{array}$ & Bag Weight & $\begin{array}{c}\text { Weight of } \\
\text { Bag+Sampte (after } \\
\text { acld wash) }\end{array}$ & $\begin{array}{l}\text { Weight of } \\
\text { Container }\end{array}$ & $\begin{array}{c}\begin{array}{c}\text { Weight of } \\
\text { Container }+ \\
\text { Ash }\end{array} \\
\end{array}$ & Weight of Ash & $\begin{array}{c}\text { Welght Loss } \\
\text { (alter lg) }\end{array}$ & $\begin{array}{l}\% \text { Lignin (dry } \\
\text { malter basis) }\end{array}$ \\
\hline & & & (g) & (g) & (a) & (a) & (g) & (g) & (a) & (a) \\
\hline$\overline{1 A}$ & 18 & June 18/02 & 0.48103173 & 0.5734 & 0.6813 & 32.7255 & 32.7577 & 0.0322 & $0.649 t$ & 15.69582863 \\
\hline $\mathrm{IB}$ & 18 & June $18 / 02$ & 0.49824322 & 0.569 & 0.6617 & 32.6318 & 32.6583 & 0.0265 & 0.6352 & 13.24723231 \\
\hline $2 \mathrm{~A}$ & 18 & June $18 / 02$ & 0.49044621 & 0.5729 & 0.7165 & 33.6051 & 33.6621 & 0.0570 & 0.6595 & 17.61703692 \\
\hline $2 B$ & 18 & June $18 / 02$ & 0.4868632 & 0.5767 & 0.6711 & 38.5213 & 38.5515 & 0.0302 & 0.6409 & 13.1455359 \\
\hline $3 A$ & 18 & June 18/02 & 0.48632626 & 0.5542 & 0.6571 & 33.8542 & 33.888 & 0.0338 & 0.6233 & 14.16920181 \\
\hline 3B & 18 & June $18 / 02$ & 0.50417045 & 0.5647 & 0.6807 & 33.8069 & 33.8517 & 0.0448 & 0.6359 & 14.08351513 \\
\hline $4 \mathrm{~A}$ & 18 & June 18/02 & 0.49952072 & 0.5932 & 0.6962 & 32.6958 & 32.7269 & 0.0311 & 0.6651 & 14.35277328 \\
\hline 48 & 18 & $\operatorname{Jun} \theta 18 / 02$ & 0.50162888 & 0.5616 & 0.6666 & 32.7818 & 32,8147 & 0.0329 & 0.6337 & 14.33450039 \\
\hline $1 \mathrm{~A}$ & 46 & July $16 / 02$ & 0.49983366 & 0.5810 & 0.7131 & 33.8708 & 33.9202 & 0.0494 & 0.6637 & 16.50534936 \\
\hline $1 \mathrm{~B}$ & 46 & Jully $16 / 02$ & 0.48965847 & 0.6170 & 0.7298 & 33.3641 & 33.4023 & 0.0382 & 0.6916 & 15.19157926 \\
\hline $2 A$ & 46 & July $16 / 02$ & 0.50342867 & 0.5682 & 0.6783 & 33.8111 & 33.85 & 0.0389 & 0.6394 & 14.10402658 \\
\hline $2 \mathrm{~B}$ & 46 & July $16 / 02$ & 0.49385763 & 0.5660 & 0.6827 & 32.4863 & 32.5264 & 0.0401 & 0.6426 & 15.4709513 \\
\hline $3 \mathrm{~A}$ & 46 & July $16 / 02$ & 0.49539412 & 0.5768 & 0.6999 & 32.4905 & 32.539 & 0.0485 & 0.6514 & 15.01849532 \\
\hline $3 \mathrm{~B}$ & 46 & July $16 / 02$ & 0.49039367 & 0.5796 & 0.6797 & 38.708 & 38.7441 & 0.0361 & 0.6436 & 13.00991002 \\
\hline $4 \mathrm{~A}$ & 46 & July $16 / 02$ & 0.48775829 & 0.5821 & 0.6931 & 33.236 & 33.2723 & 0.0363 & 0.6568 & 15.27373551 \\
\hline 48 & 46 & July $16 / 02$ & 0.49689306 & 0.5441 & 0.6587 & 33.6943 & 33.7299 & 0.0356 & $0.623 t$ & 15.86096586 \\
\hline IA & 74 & Aug 13/02 & 0.48916374 & 0.5711 & 0.7034 & 32.8745 & 32.9225 & 0.048 & 0.6554 & 17.19316146 \\
\hline $1 \mathrm{~B}$ & 74 & Aug 13/02 & 0.49990915 & 0.5735 & 0.7082 & 32.8645 & 32.911 & 0.0465 & 0.6617 & 17.6035751 \\
\hline $2 A$ & 74 & Aug $13 / 02$ & 0.49226161 & 0.5726 & 0.6995 & 33.9424 & 33.9965 & 0.0541 & 0.6454 & 14.74870114 \\
\hline $2 \mathrm{~B}$ & 74 & Aug $13 / 02$ & 0.49588022 & 0.5866 & 0.7326 & 33.1375 & 33.1988 & 0.0613 & 0.6713 & 17.03987236 \\
\hline $3 A$ & 74 & Aug $13 / 02$ & 0.50231527 & 0.5833 & 0.7197 & 39.2676 & 39.3278 & 0.0602 & 0.6595 & 15.12964092 \\
\hline $3 \mathrm{~B}$ & 74 & Aug 13/02 & 0.50618303 & 0.5806 & 0.7342 & 33.0293 & 33.1028 & 0.0735 & 0.6607 & 15.78469157 \\
\hline $4 \mathrm{~A}$ & 74 & Aug 13/02 & 0.49254788 & 0.5871 & 0.7665 & 34.3697 & 34.4405 & 0.0708 & 0.6957 & 22.00744094 \\
\hline $4 \mathrm{~B}$ & 74 & Aug $13 / 02$ & 0.50289655 & 0.5906 & 0.7553 & 33.2161 & 33.2765 & 0.0604 & 0.6949 & 20.69928191 \\
\hline $1 \mathrm{~A}$ & 109 & Sept 17/02 & 0.50029268 & 0.5933 & 0.7369 & 33.1658 & 33.2235 & 0.0577 & 0.6792 & 17.12898173 \\
\hline $1 \mathrm{~B}$ & 109 & Sept $17 / 02$ & 0.50836839 & 0.5735 & 0.7166 & 34.305 & 34.3646 & 0.0596 & 0.657 & 16.38612533 \\
\hline $2 A$ & 109 & Sept $17 / 02$ & 0.50131316 & 0.5829 & 0.7402 & 33.2375 & 33.3141 & 0.0766 & 0.6636 & 16.05755457 \\
\hline 28 & 109 & Sept $17 / 02$ & 0.50068557 & 0.5893 & 0.764 & 32.786 & 32.876 & 0.09 & 0.674 & 16.87614522 \\
\hline $3 \mathrm{~A}$ & 109 & Sept $17 / 02$ & 0.50480774 & 0.5519 & 0.7378 & 33.0442 & 33.1408 & 0.0966 & 0.6412 & 17.6521352 \\
\hline $3 \mathrm{~B}$ & 109 & Sept $17 / 02$ & 0.51272684 & 0.5696 & 0.7363 & 38.2248 & 38.3112 & 0.0864 & 0.6499 & 15.62298361 \\
\hline $4 \mathrm{~A}$ & 109 & Sept 17/02 & 0.50699641 & 0.5871 & 0.7731 & 32.9152 & 32.9932 & 0.078 & 0.6951 & 21.26192263 \\
\hline $4 \mathrm{~B}$ & 109 & Sept $17 / 02$ & 0.50042126 & 0.5684 & 0.7425 & 32.8556 & 32.9234 & 0.0678 & 0.6747 & 21.20286479 \\
\hline IA & 123 & Oct $1 / 02$ & 0.49213925 & 0.5168 & 0.6896 & 34.0983 & 34.1797 & 0.0814 & 0.6082 & 18.53570297 \\
\hline $1 \mathrm{~B}$ & 123 & Ocl 1/02 & 0.49674564 & 0.5636 & 0.7111 & 38.4015 & 38.4659 & 0.0644 & \begin{tabular}{ll|}
0.6467 \\
\end{tabular} & 16.68968885 \\
\hline $2 A$ & 123 & Oct $1 / 02$ & 0.5049396 & 0.5831 & 0.7465 & 32.8406 & 32.9221 & 0.0815 & 0.665 & 16.17986909 \\
\hline $2 B$ & 123 & Oct $1 / 02$ & 0.49667024 & 0.5681 & 0.7262 & 34.1191 & 34.2016 & 0.0825 & 0.6437 & 15.1818535 \\
\hline $3 A$ & 123 & Oct $1 / 02$ & 0.51029156 & 0.5755 & 0.7554 & 33.5769 & 33.6735 & 0.0966 & 0.6588 & 16.2850414 \\
\hline $3 B$ & 123 & Oct $1 / 102$ & 0.50749213 & 0.5692 & 0.7606 & 38.7279 & 38.8333 & 0.1054 & 0.6552 & 16.90732955 \\
\hline $4 \mathrm{~A}$ & 123 & Oct $1 / 02$ & 0.50579924 & 0.5701 & 0.7704 & 32.8726 & 32.9618 & 0.0892 & 0.6812 & 21.92629943 \\
\hline $4 \mathrm{~B}$ & 123 & Oct $1 / 02$ & 0.50289953 & 0.5797 & 0.7793 & 33.1076 & 33.1869 & 0.0793 & $\begin{array}{l}0.7 \\
\end{array}$ & 23.881458 \\
\hline IA & 137 & Oct 15/02 & 0.49696363 & 0.5689 & 0.7266 & 33.1688 & 33.2439 & 0.0751 & 0.6515 & 16.58138862 \\
\hline $1 \mathrm{~B}$ & 137 & Oct $15 / 02$ & 0.50932064 & 0.5522 & 0.724 & 34.2875 & 34.3676 & 0.0801 & 0.6439 & 17.96692167 \\
\hline $2 \mathrm{AA}$ & 137 & Oct $15 / 02$ & 0.50135137 & 0.5704 & 0.7457 & 34.2558 & 34.3447 & 0.0889 & 0.6568 & 17.1941194 \\
\hline $2 \mathrm{~B}$ & 137 & Oct $15 / 02$ & 0.50286379 & 0.5303 & 0.7016 & 32.8577 & 32.9464 & 0.0887 & 0.6129 & 16.38948892 \\
\hline $3 \mathrm{~A}$ & 137 & Oct $15 / 02$ & 0.50537689 & 0.5713 & 0.7354 & 33.0638 & 33.1493 & 0.0855 & 0.6499 & 15.51369744 \\
\hline $3 B$ & 137 & Oct 15/02 & 0.4990994 & 0.5472 & 0.706 & 38.8734 & 38.9614 & 0.088 & 0.618 & 14.14767646 \\
\hline $4 \mathrm{~A}$ & 137 & Oct $15 / 02$ & 0.49668777 & 0.5329 & 0.7061 & 33.4156 & 33.492 & 0.0764 & 0.6297 & 19.45204099 \\
\hline $4 \mathrm{~B}$ & 137 & Oct 15/02 & 0.49888165 & 0.5306 & 0.708 & 38.6008 & 38.6755 & 0.0747 & 0.6333 & 20.54930297 \\
\hline
\end{tabular}




\section{Phase 2 - Llgn!n Determinatlon}

Technician: Janice Haines Date: February 5, 2003

\begin{tabular}{l|l|} 
Blank: & 1.00034545 \\
\hline
\end{tabular}

\begin{tabular}{|c|c|c|c|c|c|c|c|c|c|c|}
\hline $\begin{array}{c}\text { Sample } \\
\text { ID }\end{array}$ & $\begin{array}{l}\text { Day } \\
\text { No. }\end{array}$ & Sample Date & $\begin{array}{c}\text { Sample Dry } \\
\text { Weight }\end{array}$ & Bag Wolght & \begin{tabular}{|c|} 
Weight of \\
Bag+Sample (after \\
acid wash)
\end{tabular} & $\begin{array}{l}\text { Woight of } \\
\text { Container }\end{array}$ & \begin{tabular}{|c|}
$\begin{array}{c}\text { Weight of } \\
\text { Container }+ \\
\text { Ash }\end{array}$ \\
\end{tabular} & Welght of Ash & $\begin{array}{c}\text { Weight Loss } \\
\text { (after ig) }\end{array}$ & $\begin{array}{l}\% \text { Llgnin (dry } \\
\text { matter basis) }\end{array}$ \\
\hline & & & $(g)$ & (a) & (a) & (G) & (g) & (a) & (a) & (g) \\
\hline $1 \mathrm{~A}$ & 158 & Nov $5 / 02$ & 0.49735235 & 0.5814 & 0.7677 & 33.5286 & 33.6246 & 0.096 & $\begin{array}{l}0.6717 \\
\end{array}$ & 18.11575894 \\
\hline $1 \mathrm{~B}$ & 158 & Nov $5 / 02$ & 0.50565086 & 0.6008 & 0.7829 & 33.3872 & 33.4784 & 0.0912 & 0.6917 & 17.93578522 \\
\hline $2 \mathrm{~A}$ & 158 & Nov $5 / 02$ & 0.50125972 & 0.5769 & 0.744 & 38.3352 & 38.4245 & 0.0893 & 0.6547 & 15.48113775 \\
\hline $2 \mathrm{~B}$ & 158 & Nov $5 / 02$ & 0.50036575 & 0.5876 & 0.7609 & 38.7031 & 38.7967 & 0.0936 & 0.6673 & 15.8877805 \\
\hline $3 \mathrm{~A}$ & 158 & Nov 5/02 & 0.49804888 & 0.5829 & 0.7653 & 39.3963 & 39.499 & 0.1027 & 0.6626 & 15.96201453 \\
\hline $3 \mathrm{~B}$ & 158 & Nov $5 / 02$ & 0.50681929 & 0.5870 & 0.7593 & 33.4979 & 33.598 & 0.1001 & 0.6592 & 14.20569822 \\
\hline $4 \mathrm{~A}$ & 158 & Nov 5/02 & 0.50236252 & 0.5751 & 0.7629 & 38.2739 & 38.362 & 0.0881 & 0.6748 & 19.80667867 \\
\hline $4 \mathrm{~B}$ & 158 & Nov $5 / 02$ & 0.50235133 & 0.5606 & 0.7391 & 32.7452 & 32.8271 & 0.0819 & 0.6572 & 19.19101906 \\
\hline IA & 180 & Nov $27 / 02$ & 0.50607027 & 0.5605 & 0.76 & 33,2722 & 33.3737 & 0.1015 & 0.6585 & 19.32663882 \\
\hline $\mathrm{IB}$ & 180 & Nov 27/02 & 0.50353311 & 0.5917 & 0.7841 & 33.1602 & 33.2561 & 0.0959 & 0.6882 & 19.12398485 \\
\hline $2 \mathrm{~A}$ & 180 & Nov $27 / 02$ & 0.49855621 & 0.5671 & 0.7461 & 33.04 & 33.1352 & 0.0952 & 0.6509 & 16.76924121 \\
\hline 28 & 180 & Nov $27 / 02$ & 0.49958088 & 0.5773 & 0.7606 & 33.5495 & 33.6466 & 0.0971 & 0.6635 & 17.21454375 \\
\hline $3 A$ & 180 & Nov $27 / 02$ & 0.50070116 & 0.5653 & 0.7491 & 32.0792 & 32.1789 & 0.0997 & 0.6494 & 16.75744355 \\
\hline $3 \mathrm{~B}$ & 180 & Nov $27 / 02$ & 0.4992749 & 0.5595 & 0.7524 & 32.8114 & 32.9201 & 0.1087 & 0.6437 & 16.82574448 \\
\hline $4 \mathrm{~A}$ & 180 & Nov $27 / 02$ & 0.5064784 & 0.6009 & 0.8063 & 33.6252 & 33.7246 & 0.0994 & 0.7069 & 20.88784357 \\
\hline $4 \bar{B}$ & 180 & Nov 27/02 & 0.50733069 & 0.5563 & 0.7626 & 34.0736 & 34.1692 & 0,0956 & 0.667 & 21.78220763 \\
\hline \multirow[t]{4}{*}{ KWSTD } & & & 0.48191145 & 0.5174 & 0.5512 & 32.8566 & 32.8657 & 0.0091 & 0.5421 & 5.088333552 \\
\hline & & & 0.48039332 & 0.5233 & 0.5556 & 34.1596 & 34.1671 & 0.0075 & 0.5481 & 5.124805614 \\
\hline & & & 0.48143704 & 0.5856 & 0.6267 & 33,6766 & 33.6915 & 0.0149 & 0.6118 & 5.400021225 \\
\hline & & & 0.48086774 & 0.5899 & 0.6196 & 32.5049 & 32.5123 & 0.0074 & 0.6122 & 4.595071573 \\
\hline \multirow[t]{4}{*}{ Blank } & & & & 0.5224 & 0.5266 & 33.0027 & 33.0082 & 0.0055 & & \\
\hline & & & & 0.5387 & 0.5532 & 32.5602 & 32.5738 & 0.0136 & & \\
\hline & & & & 0.6031 & 0.6322 & 33.7997 & 33.8224 & 0.0227 & & \\
\hline & & & & 0.5706 & 0.571 & 33.6177 & 33.6229 & 0.0052 & & \\
\hline
\end{tabular}

Blank Correctlon Factor

\begin{tabular}{|l|l|l|l|l|l|l|l|}
\hline & $\begin{array}{l}\text { Weight of } \\
\text { Material in } \\
\text { Bag }\end{array}$ & $\begin{array}{l}\text { Weight of Bag } \\
\text { after Acid } \\
\text { Wash }\end{array}$ & $\begin{array}{l}\text { Weight of } \\
\text { Container }\end{array}$ & $\begin{array}{l}\text { Weight of } \\
\text { Container }+ \\
\text { Ash }\end{array}$ & $\begin{array}{l}\text { Weight of } \\
\text { Ash }\end{array}$ & $\begin{array}{l}\text { Weight Loss } \\
\text { Acld to Ash) }\end{array}$ & Wh loss/Bag Wt \\
\hline & $(\mathrm{g})$ & $(\mathrm{g})$ & & & & & \\
\hline Blank & 0.5224 & 0.5266 & 33.0027 & 33.0082 & 0.0055 & 0.5211 & 0.997511485 \\
\hline & 0.5387 & 0.5532 & 32.5602 & 32.5738 & 0.0136 & 0.5396 & 1.001670689 \\
\hline & 0.6031 & 0.6322 & 33.7997 & 33.8224 & 0.0227 & 0.6095 & 1.010611839 \\
\hline & 0.5706 & 0.571 & 33.6177 & 33.6229 & 0.0052 & 0.5658 & 0.991587802 \\
\hline
\end{tabular}


Phase 2 - Neutral Detergent Flbre (NDF) Determination

Technician: Janice Haines, Soil Sclence Date: September 27, 2002

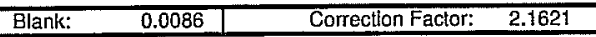

\begin{tabular}{|c|c|c|c|c|c|c|c|c|c|c|}
\hline $\begin{array}{c}\text { Sample } \\
\text { Identification }\end{array}$ & $\begin{array}{c}\text { Day of } \\
\text { Experiment }\end{array}$ & Date of Sample & $\begin{array}{c}\text { Bag Wt } \\
\text { (g) }\end{array}$ & $\begin{array}{c}\text { Wet Wt } \\
\text { (g) }\end{array}$ & $\begin{array}{c}\text { Dry Wt + Bag } \\
(\mathrm{a})\end{array}$ & $\begin{array}{c}\text { Dry Wt - Blank } \\
\text { (g) }\end{array}$ & $\%$ NDF & $\% \mathrm{DM}$ & $\begin{array}{l}\% \text { NDF (dry } \\
\text { matter basis) }\end{array}$ & $\begin{array}{l}\text { Corrected } \\
\text { \%NDF }\end{array}$ \\
\hline $1 \mathrm{~A}$ & 4 & June 4/02 & 0.5644 & 0.5027 & 0.9554 & 0.3824 & 76.0692 & 95.3451 & 79.7830 & 77.6209 \\
\hline $1 \mathrm{~B}$ & 4 & June 4/02 & 0.5583 & 0.5016 & 0.9425 & 0.3756 & 74.8804 & 95.7610 & 78.1950 & 76.0329 \\
\hline $2 \mathrm{~A}$ & 4 & June $4 / 02$ & 0.5468 & 0.5184 & 0.9226 & 0.3672 & 70.8333 & 96.6770 & 73.2681 & 71.1059 \\
\hline $2 \mathrm{~B}$ & 4 & June $4 / 02$ & 0.5727 & 0.5067 & 0.9448 & 0.3635 & 71.7387 & 96.8094 & 74.1031 & 71.9409 \\
\hline $3 A$ & 4 & June 4/02 & 0.5731 & 0.5176 & 0.9791 & 0.3974 & 76.7774 & 97.1473 & 79.0320 & 76.8698 \\
\hline 3B & 4 & June 4/02 & 0.5736 & 0.5038 & 0.9529 & 0.3707 & 73.5808 & 97.3233 & 75.6045 & 73.4424 \\
\hline $4 \mathrm{~A}$ & 4 & June 4/02 & 0.5850 & 0.5044 & 0.9337 & 0.3401 & 67.4266 & 97.1903 & 69.3759 & 67.2137 \\
\hline $4 B$ & 4 & June $4 / 02$ & 0.5721 & 0.5066 & 0.9437 & 0.3630 & 71.6542 & 97.0623 & 73.8229 & 71.6608 \\
\hline $1 \mathrm{~A}$ & 25 & June 25/02 & 0.5791 & 0.5134 & 0.9233 & 0.3356 & 65.3681 & 95.7696 & 68.2556 & 66.0935 \\
\hline $1 \mathrm{~B}$ & 25 & June 25/02 & 0.5774 & 0.5177 & 0.9391 & 0.3531 & 68.2055 & 96.2830 & 70.8386 & 68.6764 \\
\hline $2 A$ & 25 & June 25/02 & 0.5962 & 0.5126 & 0.9182 & 0.3134 & 61.1393 & 96.6839 & 63.2363 & 61.0742 \\
\hline $2 B$ & 25 & June 25/02 & 0.5697 & 0.5151 & 0.8820 & 0.3037 & 58.9594 & 96.2485 & 61.2575 & 59.0954 \\
\hline $3 A$ & 25 & June $25 / 02$ & 0.5848 & 0.5024 & 0.8966 & 0.3032 & 60.3503 & 96.0874 & 62.8077 & 60.6456 \\
\hline $3 \mathrm{~B}$ & 25 & June 25/02 & 0.5657 & 0.5048 & 0.8828 & 0.3085 & 61.1133 & 96.5246 & 63.3137 & 61.1516 \\
\hline $4 \mathrm{~A}$ & 25 & June 25/02 & 0.5894 & 0.5025 & 0.8558 & 0.2578 & 51.3035 & 96.7672 & 53.0175 & 50.8553 \\
\hline $4 \mathrm{~B}$ & 25 & June $25 / 02$ & 0.5901 & 0.5030 & 0.8896 & 0.2909 & 57.8330 & 95.7148 & 60.4222 & 58.2601 \\
\hline $1 \mathrm{~A}$ & 60 & July $30 / 02$ & 0.5639 & 0.5073 & 0.8316 & 0.2591 & 51.0743 & 97.6200 & 52.3195 & 50.1574 \\
\hline $1 \mathrm{~B}$ & 60 & July $30 / 02$ & 0.5939 & 0.5165 & 0.9094 & 0.3069 & 59.4192 & 97.7535 & 60.7847 & 58.6226 \\
\hline $2 A$ & 60 & July $30 / 02$ & 0.5692 & 0.5144 & 0.8378 & 0.2600 & 50.5443 & 98.3422 & 51.3964 & 49.2342 \\
\hline $2 \mathrm{~B}$ & 60 & July 30/02 & 0.5878 & 0.5199 & 0.8374 & 0.2410 & 46.3551 & 98.2045 & 47.2026 & 45.0405 \\
\hline $3 A$ & 60 & July $30 / 02$ & 0.5659 & 0.5128 & 0.7978 & 0.2233 & 43.5452 & 98.2522 & 44.3199 & 42.1578 \\
\hline $3 \mathrm{~B}$ & 60 & July 30/02 & 0.5924 & 0.5043 & 0.8097 & 0.2087 & 41.3841 & 98.4473 & 42.0368 & 39.8747 \\
\hline $4 \mathrm{~A}$ & 60 & July $30 / 02$ & 0.5619 & 0.5049 & 0.8385 & 0.2680 & 53.0798 & 96.5009 & 55.0045 & 52.8424 \\
\hline $4 \mathrm{~B}$ & 60 & July 30/02 & 0.5646 & 0.5055 & 0.8592 & 0.2860 & 56.5776 & 97.1069 & 58.2632 & 56.1011 \\
\hline $1 \mathrm{~A}$ & 95 & Sept 3/02 & 0.5576 & 0.5065 & 0.8202 & 0.2540 & 50.1481 & 97.4056 & 51.4838 & 49.3216 \\
\hline $1 \mathrm{~B}$ & 95 & Sept 3/02 & 0.5558 & 0.5125 & 0.8435 & 0.2791 & 54.4585 & 97.1570 & 56.0521 & 53.8900 \\
\hline $2 A$ & 95 & Sept $3 / 02$ & 0.5596 & 0.5103 & 0.7883 & 0.2201 & 43.1315 & 98.3028 & 43.8762 & 41.7140 \\
\hline $2 \mathrm{~B}$ & 95 & Sept 3/02 & 0.5697 & 0.5045 & 0.7659 & 0.1876 & 37.1853 & 98.2661 & 37.8415 & 35.6793 \\
\hline $3 \mathrm{~A}$ & 95 & Sept 3/02 & 0.5753 & 0.5006 & 0.8128 & 0.2289 & 45.7251 & 99.2233 & 46.0831 & 43.9209 \\
\hline $3 \mathrm{~B}$ & 95 & Sept $3 / 02$ & 0.5471 & 0.5093 & 0.7317 & 0.1760 & 34.5572 & 98.5891 & 35.0518 & 32.8897 \\
\hline $4 \mathrm{~A}$ & 95 & Sept 3/02 & 0.5627 & 0.5064 & 0.8345 & 0.2632 & 51.9747 & 98.4971 & 52.7678 & 50.6056 \\
\hline $4 \mathrm{~B}$ & 95 & Sept $3 / 02$ & 0.5718 & 0.5077 & 0.8573 & 0.2769 & 54.5401 & 98.4740 & 55.3852 & 53.2231 \\
\hline 1997 straw & & $22 / 04 / 02$ & 0.5911 & 0.5085 & 0.9556 & 0.3559 & 69.9902 & 94.9931 & 73.6792 & 71.5171 \\
\hline 1999 straw & & $22 / 04 / 02$ & 0.5708 & 0.5018 & 0.9412 & 0.3618 & 72.1004 & 95.7858 & 75.2726 & 73.1104 \\
\hline 2000 straw & & $22 / 04 / 02$ & 0.5627 & 0.5032 & 0.9483 & 0.3770 & 74.9205 & 96.1167 & 77.9474 & 75.7853 \\
\hline \multirow[t]{5}{*}{ KWSTD } & & & 0.5892 & 0.5114 & 0.7802 & 0.1824 & 35.6668 & 92.8907 & 38.3965 & 36.2344 \\
\hline & & & 0.5780 & 0.5159 & 0.7750 & 0.1884 & 36.5187 & 92.8907 & 39.3136 & 37.1515 \\
\hline & & & 0.5608 & 0.5083 & 0.7463 & 0.1769 & 34.8023 & 92.8907 & 37.4658 & 35.3037 \\
\hline & & & 0.5784 & 0.5141 & 0.7820 & 0.1950 & 37.9304 & 92.8907 & 40.8333 & 38.6712 \\
\hline & & & & & & & & Mean $=$ & 39.0023 & 36.8402 \\
\hline
\end{tabular}

Blank 
Phase 2 - Neutral Detergent Fibre (NDF) DetermInation

\begin{tabular}{ll|l|r|} 
Technician: Janice Haines, Soil Science & Blank: & 0.0104 \\
\hline
\end{tabular}

Date: January 30,2003

\begin{tabular}{|c|c|c|c|c|c|c|c|c|c|}
\hline $\begin{array}{c}\text { Sample } \\
\text { Identification }\end{array}$ & $\begin{array}{c}\text { Day of } \\
\text { Experiment }\end{array}$ & Date of Sample & $\begin{array}{c}\text { Bag Wt } \\
(g)\end{array}$ & $\begin{array}{l}\text { Wet Wt } \\
\text { (g) }\end{array}$ & $\begin{array}{c}\text { Dry Wt + Bag } \\
(\mathrm{g})\end{array}$ & $\begin{array}{c}\text { Dry Wt - Blank } \\
\text { (g) }\end{array}$ & $\%$ NDF & $\%$ DM & $\begin{array}{l}\% \text { NDF (dry } \\
\text { matter basis) }\end{array}$ \\
\hline $1 \mathrm{~A}$ & 18 & June $18 / 02$ & 0.5837 & 0.5014 & 0.9472 & 0.3531 & 70.4228 & 95.5945 & 73.6682 \\
\hline 18 & 18 & June $18 / 02$ & 0.5824 & 0.5143 & 0.9611 & 0.3683 & 71.6119 & 96.5027 & 74.2072 \\
\hline $2 \mathrm{~A}$ & 18 & June $18 / 02$ & 0.5676 & 0.5183 & 0.9189 & 0.3409 & 65.7727 & 96.6968 & 68.0195 \\
\hline $2 \mathrm{~B}$ & 18 & June $18 / 02$ & 0.5635 & 0.5168 & 0.8619 & 0.2880 & 55.7276 & 96.3513 & 57.8379 \\
\hline $3 \mathrm{~A}$ & 18 & June $18 / 02$ & 0.5978 & 0.5042 & 0.9106 & 0.3024 & 59.9762 & 95.6770 & 62.6861 \\
\hline 38 & 18 & June $18 / 02$ & 0.5716 & 0.5142 & 0.8851 & 0.3031 & 58.9459 & 97.4242 & 60.5044 \\
\hline $4 \mathrm{~A}$ & 18 & June $18 / 02$ & 0.5786 & 0.5110 & 0.8982 & 0.3092 & 60.5088 & 97.3914 & 62.1295 \\
\hline $4 B$ & 18 & June $18 / 02$ & 0.5757 & 0.5046 & 0.8757 & 0.2896 & 57.3920 & 97.1772 & 59.0591 \\
\hline $1 \mathrm{~A}$ & 46 & July $16 / 02$ & 0.5841 & 0.5048 & 0.8986 & 0.3041 & 60.2417 & 96.6796 & 62.3106 \\
\hline $1 \mathrm{~B}$ & 46 & July $16 / 02$ & 0.5830 & 0.5131 & 0.9131 & 0.3197 & 62.3075 & 97.4057 & 63.9670 \\
\hline $2 A$ & 46 & July $16 / 02$ & 0.5741 & 0.5038 & 0.8446 & 0.2601 & 51.6276 & 98.0005 & 52.6810 \\
\hline $2 \mathrm{~B}$ & 46 & July $16 / 02$ & 0.5885 & 0.5011 & 0.8248 & 0.2259 & 45.0808 & 96.2310 & 46.8465 \\
\hline $3 A$ & 46 & July $16 / 02$ & 0.5937 & 0.5143 & 0.8632 & 0.2591 & 50.3792 & 96.3989 & 52.2611 \\
\hline $3 B$ & 46 & July $16 / 02$ & 0.6089 & 0.5097 & 0.8293 & 0.2100 & 41.2007 & 97.4163 & 42.2934 \\
\hline $4 \mathrm{~A}$ & 46 & July $16 / 02$ & 0.5761 & 0.5059 & 0.8375 & 0.2510 & 49.6145 & 97.5517 & 50.8598 \\
\hline $4 \mathrm{~B}$ & 46 & July $16 / 02$ & 0.6051 & 0.5121 & 0.8801 & 0.2646 & 51.6696 & 96.9737 & 53.2821 \\
\hline $1 \mathrm{~A}$ & 74 & Aug $13 / 02$ & 0.5753 & 0.5047 & 0.8795 & 0.2938 & 58.2128 & 96.6918 & 60.2045 \\
\hline $1 \mathrm{~B}$ & 74 & Aug $13 / 02$ & 0.5917 & 0.5050 & 0.8888 & 0.2867 & 56.7723 & 97.5052 & 58.2249 \\
\hline $2 A$ & 74 & Aug $13 / 02$ & 0.5810 & 0.5043 & 0.8295 & 0.2381 & 47.2140 & 98.0796 & 48.1384 \\
\hline $2 \mathrm{~B}$ & 74 & Aug $13 / 02$ & 0.5749 & 0.5122 & 0.8054 & 0.2201 & 42.9715 & 98.2330 & 43.7445 \\
\hline $3 A$ & 74 & Aug 13/02 & 0.5832 & 0.5048 & 0.7870 & 0.1934 & 38.3122 & 98.4932 & 38.8983 \\
\hline $3 B$ & 74 & Aug $13 / 02$ & 0.5696 & 0.5067 & 0.7844 & 0.2044 & 40.3395 & 98.3644 & 41.0102 \\
\hline $4 \mathrm{~A}$ & 74 & Aug $13 / 02$ & 0.5784 & 0.5050 & 0.8448 & 0.2560 & 50.6931 & 97.8442 & 51.8100 \\
\hline $4 \mathrm{~B}$ & 74 & Aug $13 / 02$ & 0.5801 & 0.5083 & 0.8684 & 0.2779 & 54.6724 & 98.6072 & 55.4447 \\
\hline $1 \mathrm{~A}$ & 109 & Sept $17 / 02$ & 0.5858 & 0.5031 & 0.8061 & 0.2099 & 41.7213 & 98.3280 & 42.4308 \\
\hline $1 \mathrm{~B}$ & 109 & Sept $17 / 02$ & 0.6029 & 0.5120 & 0.8743 & 0.2610 & 50.9766 & 98.8274 & 51.5814 \\
\hline $2 A$ & 109 & Sept $17 / 02$ & 0.5811 & 0.5059 & 0.7836 & 0.1921 & 37.9719 & 98.9564 & 38.3724 \\
\hline $2 \mathrm{~B}$ & 109 & Sept $17 / 02$ & 0.5768 & 0.5001 & 0.7629 & 0.1757 & 35.1330 & 99.3621 & 35.3585 \\
\hline $3 A$ & 109 & Sept $17 / 02$ & 0.5844 & 0.5098 & 0.8059 & 0.2111 & 41.4084 & 98.9237 & 41.8589 \\
\hline $3 B$ & 109 & Sept $17 / 02$ & 0.5768 & 0.5054 & 0.7860 & 0.1988 & 39.3352 & 99.8689 & 39.3868 \\
\hline $4 \mathrm{~A}$ & 109 & Sept $17 / 02$ & 0.5783 & 0.5092 & 0.8344 & 0.2457 & 48.2522 & 99.2554 & 48.6142 \\
\hline $4 \mathrm{~B}$ & 109 & Sept 17102 & 0.5457 & 0.5001 & 0.8255 & 0.2694 & 53.8692 & 98.8584 & 54.4913 \\
\hline $1 \mathrm{~A}$ & 123 & Oct $1 / 02$ & 0.5713 & 0.5082 & 0.8125 & 0.2308 & 45.4152 & 97.7631 & 46.4543 \\
\hline $1 \mathrm{~B}$ & 123 & Oct $1 / 02$ & 0.5466 & 0.5108 & 0.8149 & 0.2579 & 50.4894 & 97.7269 & 51.6638 \\
\hline $2 \mathrm{~A}$ & 123 & Oct $1 / 02$ & 0.5799 & 0.5119 & 0.8259 & 0.2356 & 46.0246 & 98.3521 & 46.7958 \\
\hline $2 B$ & 123 & Oat $1 / 02$ & 0.5768 & 0.5067 & 0.8163 & 0.2291 & 45.2141 & 98.8792 & 45.7266 \\
\hline $3 \mathrm{~A}$ & 123 & Oct $1 / 02$ & 0.5875 & 0.5107 & 0.8206 & 0.2227 & 43.6068 & 99.1820 & 43.9664 \\
\hline $3 \mathrm{~B}$ & 123 & Oct $1 / 02$ & 0.5697 & 0.5073 & 0.7760 & 0.1959 & 38.6162 & 99.6255 & 38.7614 \\
\hline $4 \mathrm{~A}$ & 123 & Oct $1 / 02$ & 0.5340 & 0.5040 & 0.7765 & 0.2321 & 46.0516 & 98.8662 & 46.5797 \\
\hline $4 \mathrm{~B}$ & 123 & Oct $1 / 02$ & 0.5424 & 0.5097 & 0.8181 & 0.2653 & 52.0502 & 98.1267 & 53.0439 \\
\hline
\end{tabular}


Phase 2 - Neutral Detergent Flbre (NDF) Determination

\begin{tabular}{ll|r|r|} 
Technician: Janice Haines, Soll Sclence & Blank: & 0.0104 \\
\hline
\end{tabular}

Date: January 30,2003

\begin{tabular}{|c|c|c|c|c|c|c|c|c|c|}
\hline $\begin{array}{c}\text { Sample } \\
\text { Identification }\end{array}$ & $\begin{array}{c}\text { Day of } \\
\text { Experiment }\end{array}$ & Date of Sample & $\begin{array}{l}\text { Bag Wt } \\
\text { (g) }\end{array}$ & $\begin{array}{c}\text { Wet Wt } \\
\text { (g) }\end{array}$ & $\begin{array}{c}\text { Dry } W t+B a g \\
\text { (a) }\end{array}$ & $\begin{array}{c}\text { Dry Wt - Blank } \\
\text { (g) }\end{array}$ & $\%$ NDF & $\% \mathrm{DM}$ & $\begin{array}{l}\% \text { NDF (dry } \\
\text { matter basis) }\end{array}$ \\
\hline $1 \mathrm{~A}$ & 137 & Oct $15 / 02$ & 0.5705 & 0.5072 & 0.7803 & 0.1994 & 39.3139 & 99.1152 & 39.6648 \\
\hline $1 \mathrm{~B}$ & 137 & Oct $15 / 02$ & 0.5251 & 0.5077 & 0.7429 & 0.2074 & 40.8509 & 99.6519 & 40.9936 \\
\hline $2 \mathrm{~A}$ & 137 & Oat $15 / 02$ & 0.5874 & 0.5059 & 0.8374 & 0.2396 & 47.3611 & 99.4153 & 47.6397 \\
\hline $2 \mathrm{~B}$ & 137 & Oct $15 / 02$ & 0.5256 & 0.5052 & 0.7659 & 0.2299 & 45.5067 & 99.8340 & 45.5824 \\
\hline $3 \mathrm{~A}$ & 137 & Oct $15 / 02$ & 0.5632 & 0.5150 & 0.8157 & 0.2421 & 47.0097 & 99.6602 & 47.1700 \\
\hline $3 \mathrm{~B}$ & 137 & Oct $15 / 02$ & 0.5832 & 0.5035 & 0.8004 & 0.2068 & 41.0725 & 99.4816 & 41.2865 \\
\hline $4 \mathrm{~A}$ & 137 & Oct $15 / 02$ & 0.5730 & 0.5124 & 0.8334 & 0.2500 & 48.7900 & 99.1591 & 49.2038 \\
\hline $4 \mathrm{~B}$ & 137 & Oct $15 / 02$ & 0.5779 & 0.5005 & 0.8459 & 0.2576 & 51.4685 & 99.2010 & 51.8831 \\
\hline $1 \mathrm{~A}$ & 158 & Nov $5 / 02$ & 0.6201 & 0.5074 & 0.8197 & 0.1892 & 37.2881 & 98.6223 & 37.8090 \\
\hline $1 \mathrm{~B}$ & 158 & Nov $5 / 02$ & 0.6252 & 0.5063 & 0.8474 & 0.2118 & 41.8329 & 99.5964 & 42.0024 \\
\hline $2 \mathrm{~A}$ & 158 & Nov $5 / 02$ & 0.5671 & 0.5034 & 0.7614 & 0.1839 & 36.5316 & 99.2790 & 36.7969 \\
\hline $2 \mathrm{~B}$ & 158 & Nov $5 / 02$ & 0.5780 & 0.5099 & 0.7930 & 0.2046 & 40.1255 & 99.3972 & 40.3688 \\
\hline $3 \mathrm{~A}$ & 158 & Nov $5 / 02$ & 0.5348 & 0.5052 & 0.7477 & 0.2025 & 40.0831 & 99.0157 & 40.4816 \\
\hline $3 \mathrm{~B}$ & 158 & Nov $5 / 02$ & 0.5837 & 0.5089 & 0.7802 & 0.1861 & 36.5691 & 99.3763 & 36.7986 \\
\hline $4 \mathrm{~A}$ & 158 & Nov $5 / 02$ & 0.5596 & 0.5081 & 0.8010 & 0.2310 & 45.4635 & 98.9877 & 45.9284 \\
\hline $4 B$ & 158 & Nov $5 / 02$ & 0.5730 & 0.5035 & 0.8180 & 0.2346 & 46.5938 & 98.7714 & 47.1734 \\
\hline $1 \mathrm{~A}$ & 180 & Nov $27 / 02$ & 0.6076 & 0.5020 & 0.8359 & 0.2179 & 43.4064 & 99.4830 & 43.6319 \\
\hline $1 \mathrm{~B}$ & 180 & Nov $27 / 02$ & 0.5580 & 0.5074 & 0.7793 & 0.2109 & 41.5648 & 99.3554 & 41.8345 \\
\hline $2 A$ & 180 & Nov $27 / 02$ & 0.5868 & 0.5082 & 0.8200 & 0.2228 & 43.8410 & 99.7112 & 43.9680 \\
\hline $2 \mathrm{~B}$ & 180 & Nov $27 / 02$ & 0.6062 & 0.5088 & 0.8397 & 0.2231 & 43.8483 & 99.4983 & 44.0694 \\
\hline $3 A$ & 180 & Nov $27 / 02$ & 0.6052 & 0.5034 & 0.8331 & 0.2175 & 43.2062 & 99.7413 & 43.3183 \\
\hline $3 \mathrm{~B}$ & 180 & Nov $27 / 02$ & 0.5737 & 0.5024 & 0.7884 & 0.2043 & 40.6648 & 99.6358 & 40.8135 \\
\hline $4 A$ & 180 & Nov 27102 & 0.5921 & 0.5059 & 0.8334 & 0.2309 & 45.6414 & 99.6809 & 45.7876 \\
\hline 48 & 180 & Nov $27 / 02$ & 0.5736 & 0.5017 & 0.8126 & 0.2286 & 45.5651 & 99.6525 & 45.7240 \\
\hline
\end{tabular}

\begin{tabular}{|c|c|c|c|c|c|c|c|}
\hline KWSTD & 0.5725 & 0.5088 & 0.7646 & 0.1817 & 35.7115 & 94.8831 & 37.6373 \\
\hline \multirow{2}{*}{} & 0.5829 & 0.5047 & 0.7699 & 0.1766 & 34.9911 & 94.8831 & 36.8781 \\
\cline { 2 - 9 } & 0.5983 & 0.5000 & 0.7851 & 0.1764 & 35.2800 & 94.8831 & 37.1826 \\
\cline { 2 - 8 } & 0.5812 & 0.5002 & 0.7628 & 0.1712 & 34.2263 & 94.8831 & 36.0721 \\
\hline
\end{tabular}

\begin{tabular}{|c|c|c|c|c|}
\hline Blank & 0.5504 & & 0.5641 & 0.0137 \\
\hline & 0.5907 & & 0.6008 & 0.0101 \\
\hline & 0.5517 & & 0.5614 & 0.0097 \\
\hline & 0.574 & & 0.5821 & 0.0081 \\
\hline \multicolumn{3}{|r|}{} \\
\cline { 2 - 4 }
\end{tabular}




\section{Phase 2 - Lab Dry Matter Determination}

Technician: Janice Haines/Scott Chapman

Date: January 30, 2003

\begin{tabular}{|c|c|c|c|c|c|c|c|c|}
\hline Samp ID & Day No. & Tube \# & Date & $\begin{array}{c}\text { Tin Wt } \\
\text { (g) }\end{array}$ & $\begin{array}{l}\text { Wet Wt } \\
\text { (g) }\end{array}$ & $\begin{array}{l}\text { Dry Wt } \\
\text { Tin }(g)\end{array}$ & $\begin{array}{l}\text { Dry Wt } \\
\text { (g) }\end{array}$ & $\% \mathrm{DM}$ \\
\hline $1 \mathrm{~A}$ & 18 & 1 & June $18 / 02$ & 18.0143 & 0.5130 & 18.5047 & 0.4904 & 95.5945 \\
\hline $1 B$ & 18 & 2 & June $18 / 02$ & 17.6913 & 0.5833 & 18.2542 & 0.5629 & 96.5027 \\
\hline $2 A$ & 18 & 3 & June 18/02 & 16.7100 & 0.5419 & 17.2340 & 0.5240 & 96.6968 \\
\hline $2 B$ & 18 & 6 & June $18 / 02$ & 17.4719 & 0.7674 & 18.2113 & 0.7394 & 96.3513 \\
\hline $3 A$ & 18 & 13 & June $18 / 02$ & 19.4900 & 0.5251 & 19.9924 & 0.5024 & 95.6770 \\
\hline $3 \mathrm{~B}$ & 18 & 14 & June $18 / 02$ & 17.0918 & 0.5940 & 17.6705 & 0.5787 & 97.4242 \\
\hline $4 \mathrm{~A}$ & 18 & 15 & June $18 / 02$ & 18.3746 & 0.6172 & 18.9757 & 0.6011 & 97.3914 \\
\hline $4 \mathrm{~B}$ & 18 & 17 & June 18/02 & 17.6455 & 0.6483 & 18.2755 & 0.6300 & 97.1772 \\
\hline $1 \mathrm{~A}$ & 46 & 20 & July $16 / 02$ & 20.1668 & 0.6174 & 20.7637 & 0.5969 & 96.6796 \\
\hline $1 \mathrm{~B}$ & 46 & 27 & July $16 / 02$ & 17.6582 & 0.5435 & 18.1876 & 0.5294 & 97.4057 \\
\hline $2 \mathrm{~A}$ & 46 & A30 & July $16 / 02$ & 12.4840 & 0.7702 & 13.2388 & 0.7548 & 98.0005 \\
\hline $2 \mathrm{~B}$ & 46 & $32 \mathrm{~A}$ & July $16 / 02$ & 12.6582 & 0.7774 & 13.4063 & 0.7481 & 96.2310 \\
\hline $3 A$ & 46 & 32 & July $16 / 02$ & 17.4367 & 0.6748 & 18.0872 & 0.6505 & 96.3989 \\
\hline $3 \mathrm{~B}$ & 46 & $37 \mathrm{~B}$ & July $16 / 02$ & 12.6533 & 0.5496 & 13.1887 & 0.5354 & 97.4163 \\
\hline $4 \mathrm{~A}$ & 46 & 39 & July $16 / 02$ & 19.7528 & 0.5759 & 20.3146 & 0.5618 & 97.5517 \\
\hline $4 \mathrm{~B}$ & 46 & 40 & July $16 / 02$ & 17.2822 & 0.6873 & 17.9487 & 0.6665 & 96.9737 \\
\hline $1 \mathrm{~A}$ & 74 & 42 & Aug 13/02 & 18.1382 & 0.5441 & 18.6643 & 0.5261 & 96.6918 \\
\hline $1 B$ & 74 & $42 B$ & Aug $13 / 02$ & 17.6081 & 0.6253 & 18.2178 & 0.6097 & 97.5052 \\
\hline $2 \mathrm{~A}$ & 74 & 43 & Aug $13 / 02$ & 17.2925 & 0.7134 & 17.9922 & 0.6997 & 98.0796 \\
\hline $2 \mathrm{~B}$ & 74 & $D$ & Aug 13/02 & 12.5171 & 0.8489 & 13.3510 & 0.8339 & 98.2330 \\
\hline $3 \mathrm{~A}$ & 74 & A36 & Aug $13 / 02$ & 12.0724 & 0.6902 & 12.7522 & 0.6798 & 98.4932 \\
\hline $3 B$ & 74 & QK & Aug 13/02 & 17.6866 & 0.9721 & 18.6428 & 0.9562 & 98.3644 \\
\hline $4 \mathrm{~A}$ & 74 & $T$ & Aug 13/02 & 18.6043 & 0.9463 & 19.5302 & 0.9259 & 97.8442 \\
\hline $4 \mathrm{~B}$ & 74 & $\mathrm{~B} 13$ & Aug $13 / 02$ & 12.6356 & 0.7395 & 13.3648 & 0.7292 & 98.6072 \\
\hline $1 \mathrm{~A}$ & 109 & B3 & Sept 17/02 & 12.7425 & 0.6519 & 13.3835 & 0.6410 & 98.3280 \\
\hline $1 \mathrm{~B}$ & 109 & $\mathrm{~B} 27$ & Sept $17 / 02$ & 12.6006 & 0.7505 & 13.3423 & 0.7417 & 98.8274 \\
\hline $2 A$ & 109 & B27 & Sept 17/02 & 18.0038 & 0.9199 & 18.9141 & 0.9103 & 98.9564 \\
\hline $2 B$ & 109 & $J$ & Sept 17/02 & 17.6052 & 0.9876 & 18.5865 & 0.9813 & 99.3621 \\
\hline $3 A$ & 109 & $\mathrm{R}$ & Sept $17 / 02$ & 17.5983 & 0.7433 & 18.3336 & 0.7353 & 98.9237 \\
\hline $3 B$ & 109 & $F$ & Sept $17 / 02$ & 17.0290 & 0.6864 & 17.7145 & 0.6855 & 99.8689 \\
\hline $4 \mathrm{~A}$ & 109 & $E$ & Sept 17/02 & 17.8728 & 0.6849 & 18.5526 & 0.6798 & 99.2554 \\
\hline $4 \mathrm{~B}$ & 109 & $\mathrm{G}$ & Sept 17/02 & 17.4400 & 0.5869 & 18.0202 & 0.5802 & 98.8584 \\
\hline
\end{tabular}


Phase 2 - Lab Dry Matter Determination

Technician: Janice Haines/Scott Chapman

Date: January 30, 2003

\begin{tabular}{|c|c|c|c|c|c|c|c|c|}
\hline Samp ID & Day No. & Tube \# & Date & $\begin{array}{c}\operatorname{Tin} W t \\
\text { (g) }\end{array}$ & $\begin{array}{l}\text { Wet Wt } \\
\text { (g) }\end{array}$ & $\begin{array}{l}\text { Dry Wt } \\
\operatorname{Tin}(g)\end{array}$ & $\begin{array}{l}\text { Dry Wt } \\
\text { (g) }\end{array}$ & $\% \mathrm{DM}$ \\
\hline $1 \mathrm{~A}$ & 123 & V & Oct $1 / 02$ & 18.0210 & 0.6795 & 18.6853 & 0.6643 & 97.7631 \\
\hline $1 \mathrm{~B}$ & 123 & $Y$ & Oct $1 / 02$ & 19.5762 & 0.5543 & 20.1179 & 0.5417 & 97.7269 \\
\hline $2 A$ & 123 & $3 A$ & Oct $1 / 02$ & 12.6929 & 0.6311 & 13.3136 & 0.6207 & 98.3521 \\
\hline $2 \mathrm{~B}$ & 123 & 9 & Oct $1 / 02$ & 14.5394 & 0.8030 & 15.3334 & 0.7940 & 98.8792 \\
\hline $3 \mathrm{~A}$ & 123 & 19 & Oct $1 / 02$ & 17.9698 & 0.7213 & 18.6852 & 0.7154 & 99.1820 \\
\hline $3 \mathrm{~B}$ & 123 & $21 \mathrm{~A}$ & Oct $1 / 02$ & 17.5525 & 0.6942 & 18.2441 & 0.6916 & 99.6255 \\
\hline $4 A$ & 123 & 26 & Oct $1 / 02$ & 17.2259 & 0.7673 & 17.9845 & 0.7586 & 98.8662 \\
\hline $4 \mathrm{~B}$ & 123 & 28 & Oct $1 / 02$ & 20.1275 & 0.6833 & 20.7980 & 0.6705 & 98.1267 \\
\hline $1 \mathrm{~A}$ & 137 & 30 & Oct $15 / 02$ & 18.0605 & 0.5538 & 18.6094 & 0.5489 & 99.1152 \\
\hline $1 \mathrm{~B}$ & 137 & 31 & Oct 15/02 & 17.8597 & 0.6032 & 18.4608 & 0.6011 & 99.6519 \\
\hline $2 \mathrm{~A}$ & 137 & 35 & Oct $15 / 02$ & 16.7326 & 0.5986 & 17.3277 & 0.5951 & 99.4153 \\
\hline $2 \mathrm{~B}$ & 137 & $36 \mathrm{~A}$ & Oct $15 / 02$ & 17.6370 & 0.6626 & 18.2985 & 0.6615 & 99.8340 \\
\hline $3 \mathrm{~A}$ & 137 & $36 \mathrm{~B}$ & Oct $15 / 02$ & 17.4053 & 0.5003 & 17.9039 & 0.4986 & 99.6602 \\
\hline $3 B$ & 137 & 37 & Oct $15 / 02$ & 17.3362 & 0.6945 & 18.0271 & 0.6909 & 99.4816 \\
\hline $4 \mathrm{~A}$ & 137 & 38 & Oct $15 / 02$ & 19.0669 & 0.8443 & 19.9041 & 0.8372 & 99.1591 \\
\hline $4 \mathrm{~B}$ & 137 & 41 & Oct $15 / 02$ & 19.6988 & 0.6633 & 20.3568 & 0.6580 & 99.2010 \\
\hline $1 \mathrm{~A}$ & 158 & B35 & Nov $5 / 02$ & 12.6666 & 0.5081 & 13.1677 & 0.5011 & 98.6223 \\
\hline $1 \mathrm{~B}$ & 158 & B36 & Nov $5 / 02$ & 18.0332 & 0.5203 & 18.5514 & 0.5182 & 99.5964 \\
\hline $2 A$ & 158 & $\mathrm{~A} 22$ & Nov $5 / 02$ & 12.7676 & 0.7351 & 13.4974 & 0.7298 & 99.2790 \\
\hline $2 \mathrm{~B}$ & 158 & $M$ & Nov $5 / 02$ & 17.1732 & 0.8793 & 18.0472 & 0.8740 & 99.3972 \\
\hline $3 A$ & 158 & $\mathrm{P}$ & Nov $5 / 02$ & 16.1948 & 0.5994 & 16.7883 & 0.5935 & 99.0157 \\
\hline 3B & 158 & $Q$ & Nov $5 / 02$ & 16.4588 & 0.6574 & 17.1121 & 0.6533 & 99.3763 \\
\hline $4 \mathrm{~A}$ & 158 & $\mathrm{U}$ & Nov $5 / 02$ & 17.4834 & 0.7310 & 18.2070 & 0.7236 & 98.9877 \\
\hline $4 \mathrm{~B}$ & 158 & 0 & Nov $5 / 02$ & 17.7298 & 0.7244 & 18.4453 & 0.7155 & 98.7714 \\
\hline $1 \mathrm{~A}$ & 180 & 16 & Nov $27 / 02$ & 20.2541 & 0.8318 & 21.0816 & 0.8275 & 99.4830 \\
\hline $1 \mathrm{~B}$ & 180 & 25 & Nov $27 / 02$ & 18.1554 & 0.8222 & 18.9723 & 0.8169 & 99.3554 \\
\hline $2 A$ & 180 & 33 & Nov $27 / 02$ & 19.4669 & 1.0043 & 20.4683 & 1.0014 & 99.7112 \\
\hline $2 \mathrm{~B}$ & 180 & 34 & Nov $27 / 02$ & 17.9362 & 0.7574 & 18.6898 & 0.7536 & 99.4983 \\
\hline $3 A$ & 180 & 44 & Nov $27 / 02$ & 17.4891 & 0.6957 & 18.1830 & 0.6939 & 99.7413 \\
\hline $3 B$ & 180 & 46 & Nov $27 / 02$ & 17.3522 & 0.6864 & 18.0361 & 0.6839 & 99.6358 \\
\hline $4 \mathrm{~A}$ & 180 & 50 & Nov $27 / 02$ & 17.4177 & 0.8460 & 18.2610 & 0.8433 & 99.6809 \\
\hline $4 \mathrm{~B}$ & 180 & 72 & Nov $27 / 02$ & 16.9521 & 0.6618 & 17.6116 & 0.6595 & 99.6525 \\
\hline KWSTD & & & $\operatorname{Jan} 30 / 03$ & 12.3535 & 0.5436 & 12.8694 & 0.5159 & 94.9043 \\
\hline & & & $\operatorname{Jan} 30 / 03$ & 19.6473 & 1.0140 & 20.6092 & 0.9619 & 94.8619 \\
\hline
\end{tabular}


Phase 2 - Acld Delergent Fibre (ADF) Determination

Technician: Janice Haines, Soil Science Date: September 27, 2002

Blank 1: $\quad 0.0070$

Blank 2: $\quad 0.0013$

\begin{tabular}{|c|c|c|c|c|c|c|c|c|c|}
\hline $\begin{array}{c}\text { Sample } \\
\text { Identification }\end{array}$ & $\begin{array}{c}\text { Day of } \\
\text { Experiment }\end{array}$ & $\begin{array}{l}\text { Date of } \\
\text { Sample }\end{array}$ & Bag Wt & Wet Wt & Dry $\mathrm{Wt}+\mathrm{Bag}$ & Dry Wt - Blank & $\%$ ADF & $\%$ DM & $\begin{array}{c}\% \text { ADF (dry matter } \\
\text { basis) }\end{array}$ \\
\hline & & & (g) & (g) & (g) & (g) & & & \\
\hline $1 \mathrm{~A}$ & 4 & June 4/02 & 0.5950 & 0.5119 & 0.8869 & 0.2849 & 55.6505 & 95.3451 & 58.3674 \\
\hline 18 & 4 & June 4/02 & 0.5911 & 0.5138 & 0.8893 & 0.2912 & 56.6709 & 95.7610 & 59.1795 \\
\hline $2 A$ & 4 & June $4 / 02$ & 0.5906 & 0.5102 & 0.8695 & 0.2719 & 53.2879 & 96.6770 & 55.1196 \\
\hline $2 B$ & 4 & June 4/02 & 0.5769 & 0.5031 & 0.8488 & 0.2649 & 52.6486 & 96.8094 & 54.3838 \\
\hline $3 A$ & 4 & June 4/02 & 0.5683 & 0.5107 & 0.8645 & 0.2892 & 56.6193 & 97.1473 & 58.2819 \\
\hline $3 B$ & 4 & June 4/02 & 0.5507 & 0.5054 & 0.8340 & 0.2763 & 54.6646 & 97.3233 & 56.1681 \\
\hline $4 \mathrm{~A}$ & 4 & June $4 / 02$ & 0.6098 & 0.5161 & 0.9265 & 0.3097 & 60.0029 & 97.1903 & 61.7375 \\
\hline $4 \mathrm{~B}$ & 4 & June 4/02 & 0.5422 & 0.5037 & 0.8327 & 0.2835 & 56.2785 & 97.0623 & 57.9819 \\
\hline $1 \mathrm{~A}$ & 25 & June 25/02 & 0.6012 & 0.5127 & 0.8845 & 0.2763 & 53.8863 & 95.7696 & 56.2666 \\
\hline $1 \mathrm{~B}$ & 25 & June 25/02 & 0.5676 & 0.5117 & 0.8499 & 0.2810 & 54.9150 & 96.2830 & 57.0350 \\
\hline $2 A$ & 25 & June 25/02 & 0.5939 & 0.5133 & 0.8675 & 0.2666 & 51.9336 & 96.6839 & 53.7148 \\
\hline $2 \mathrm{~B}$ & 25 & June 25/02 & 0.5527 & 0.5112 & 0.7877 & 0.2280 & 44.5960 & 96.2485 & 46.3343 \\
\hline $3 A$ & 25 & June 25/02 & 0.5956 & 0.5032 & 0.8261 & 0.2235 & 44.4167 & 96.0874 & 46.2254 \\
\hline $3 \mathrm{~B}$ & 25 & June 25/02 & 0.5885 & 0.5052 & 0.8315 & 0.2360 & 46.7092 & 96.5246 & 48.3910 \\
\hline $4 \mathrm{~A}$ & 25 & June $25 / 02$ & 0.5636 & 0.5114 & 0.8158 & 0.2452 & 47.9419 & 96.7672 & 49.5436 \\
\hline $4 \mathrm{~B}$ & 25 & June 25/02 & 0.6197 & 0.5030 & 0.8589 & 0.2322 & 46.1581 & 95.7148 & 48.2245 \\
\hline $1 \mathrm{~A}$ & 60 & July $30 / 02$ & 0.5891 & 0.5099 & 0.8329 & 0.2368 & 46.4356 & 97.6200 & 47.5677 \\
\hline $1 B$ & 60 & July $30 / 02$ & 0.5756 & 0.5029 & 0.8595 & 0.2769 & 55.0557 & 97.7535 & 56.3209 \\
\hline $2 \mathrm{~A}$ & 60 & July $30 / 02$ & 0.5588 & 0.5110 & 0.7872 & 0.2214 & 43.3219 & 98.3422 & 44.0522 \\
\hline $2 \mathrm{~B}$ & 60 & July $30 / 02$ & 0.5564 & 0.5063 & 0.7669 & 0.2035 & 40.1886 & 98.2045 & 40.9234 \\
\hline $3 \mathrm{~A}$ & 60 & July $30 / 02$ & 0.5912 & 0.5020 & 0.7918 & 0.1936 & 38.5608 & 98.2522 & 39.2467 \\
\hline $3 B$ & 60 & July $30 / 02$ & 0.6016 & 0.5023 & 0.8021 & 0.1935 & 38.5178 & 98.4473 & 39.1253 \\
\hline $4 \mathrm{~A}$ & 60 & July $30 / 02$ & 0.6016 & 0.5150 & 0.8765 & 0.2679 & 52.0146 & 96.5009 & 53.9006 \\
\hline $4 B$ & 60 & July $30 / 02$ & 0.5836 & 0.5134 & 0.8578 & 0.2672 & 52.0403 & 97.1069 & 53.5907 \\
\hline $1 \mathrm{~A}$ & 95 & Sept 3/02 & 0.5510 & 0.5119 & 0.8066 & 0.2543 & 49.6777 & 97.4056 & 51.0008 \\
\hline $1 \mathrm{~B}$ & 95 & Sept $3 / 02$ & 0.5677 & 0.5070 & 0.8177 & 0.2430 & 47.9241 & 97.1570 & 49.3264 \\
\hline $2 A$ & 85 & Sept 3/02 & 0.6100 & 0.5144 & 0.8365 & 0.2195 & 42.6662 & 98.3028 & 43.4028 \\
\hline 28 & 95 & Sept 3/02 & 0.5659 & 0.5047 & 0.7733 & 0.2061 & 40.8361 & 98.2661 & 41.5567 \\
\hline $3 A$ & 95 & Sept 3/02 & 0.5720 & 0.5086 & 0.8202 & 0.2412 & 47.4194 & 99.2233 & 47.7906 \\
\hline $3 B$ & 95 & Sept 3/02 & 0.5839 & 0.5157 & 0.8189 & 0.2337 & 45.3170 & 98.5891 & 45.9656 \\
\hline $4 A$ & 95 & Sept 3/02 & 0.5982 & 0.5127 & 0.8681 & 0.2629 & 51.2727 & 98.4971 & 52.0550 \\
\hline $4 \mathrm{~B}$ & 95 & Sept 3/02 & 0.5715 & 0.5140 & 0.8509 & 0.2781 & 54.1051 & 98.4740 & 54.9435 \\
\hline 1997 straw & & April 22/02 & 0.5795 & 0.5051 & 0.8307 & 0.2442 & 48.3419 & 94.9931 & 50.8899 \\
\hline 1999 straw & & April 22/02 & 0.5691 & 0.5045 & 0.8344 & 0.2583 & 51.1943 & 95.7858 & 53.4466 \\
\hline 2000 straw & & Aprit 22/02 & 0.5690 & 0.5009 & 0.8511 & 0.2751 & 54.9162 & 96.1167 & 57.1349 \\
\hline
\end{tabular}

\begin{tabular}{|l|c|c|c|c|c|c|c|}
\hline KWSTD & 0.5569 & 0.5011 & 0.6878 & 0.1239 & 24.7206 & 92.8907 & 26.6126 \\
\hline \multirow{3}{*}{} & 0.5309 & 0.5125 & 0.6764 & 0.1385 & 27.0195 & 92.8907 & 29.0874 \\
\cline { 2 - 8 } & 0.5681 & 0.5059 & 0.6992 & 0.1241 & 24.5256 & 92.8907 & 26.4026 \\
\cline { 2 - 8 } & 0.5856 & 0.5084 & 0.7492 & 0.1566 & 30.7976 & 92.8907 & 33.1546 \\
\hline
\end{tabular}

\begin{tabular}{|l|l|l|l|r|}
\hline Blank & 0.6377 & & 0.6423 & 0.0046 \\
\hline & 0.5617 & & 0.5710 & 0.0093 \\
\hline & 0.5394 & & 0.5420 & 0.0026 \\
\hline & 0.5399 & & 0.5515 & 0.0116 \\
\hline
\end{tabular}


Phase 2 - Acld Detergent Fibre (ADF) Determination

Technician: Janice Haines, Soil Science Date: January 30,2003

Blank: 0.0216

\begin{tabular}{|c|c|c|c|c|c|c|c|c|c|}
\hline $\begin{array}{c}\text { Sample } \\
\text { Identification }\end{array}$ & $\begin{array}{c}\text { Day of } \\
\text { Experiment }\end{array}$ & $\begin{array}{l}\text { Date of } \\
\text { Sample }\end{array}$ & Bag Wt & Wet Wt & Dry $W t+B a g$ & Dry Wt - Blank & $\%$ ADF & $\% \mathrm{DM}$ & $\begin{array}{l}\% \text { ADF (dry } \\
\text { matter basis) }\end{array}$ \\
\hline & & & (g) & (g) & (g) & (g) & & & \\
\hline $1 \mathrm{~A}$ & 18 & \begin{tabular}{|l|} 
June $18 / 02$ \\
\end{tabular} & 0.5734 & 0.5032 & 0.8864 & 0.2914 & 57.9044 & 95.5945 & 60.5729 \\
\hline $1 B$ & 18 & June $18 / 02$ & 0.5690 & 0.5163 & 0.8737 & 0.2831 & 54.8276 & 96.5027 & 56.8146 \\
\hline $2 A$ & 18 & June $18 / 02$ & 0.5729 & 0.5072 & 0.9221 & 0.3276 & 64.5850 & 96.6968 & 66.7912 \\
\hline $2 \mathrm{~B}$ & 18 & June $18 / 02$ & 0.5767 & 0.5053 & 0.8360 & 0.2377 & 47.0364 & 96.3513 & 48.8176 \\
\hline $3 \mathrm{~A}$ & 18 & June $18 / 02$ & 0.5542 & 0.5083 & 0.8057 & 0.2299 & 45.2243 & 95.6770 & 47.2677 \\
\hline $3 B$ & 18 & June $18 / 02$ & 0.5647 & 0.5175 & 0.8294 & 0.2431 & 46.9710 & 97.4242 & 48.2129 \\
\hline $4 \mathrm{~A}$ & 18 & June $18 / 02$ & 0.5932 & 0.5129 & 0.8494 & 0.2346 & 45.7350 & 97.3914 & 46.9600 \\
\hline $4 B$ & 18 & June 18/02 & 0.5616 & 0.5162 & 0.8257 & 0.2425 & 46.9731 & 97.1772 & 48.3375 \\
\hline $1 \mathrm{~A}$ & 46 & July $16 / 02$ & 0.5810 & 0.5170 & 0.9001 & 0.2975 & 57.5387 & 96.6796 & 59.5148 \\
\hline $1 B$ & 46 & July 16/02 & 0.6170 & 0.5027 & 0.8969 & 0.2583 & 51.3776 & 97.4057 & 52.7459 \\
\hline $2 A$ & 46 & July $16 / 02$ & 0.5682 & 0.5137 & 0.8035 & 0.2137 & 41.5953 & 98.0005 & 42.4439 \\
\hline $2 B$ & 46 & July $16 / 02$ & 0.5660 & 0.5132 & 0.7967 & 0.2091 & 40.7395 & 96.2310 & 42.3351 \\
\hline $3 \mathrm{~A}$ & 46 & July $16 / 02$ & 0.5768 & 0.5139 & 0.8100 & 0.2116 & 41.1705 & 96.3989 & 42.7084 \\
\hline $3 \mathrm{~B}$ & 46 & July $16 / 02$ & 0.5796 & 0.5034 & 0.7687 & 0.1675 & 33.2688 & 97.4163 & 34.1511 \\
\hline $4 \mathrm{~A}$ & 46 & July 16/02 & 0.5821 & 0.5000 & 0.8128 & 0.2091 & 41.8150 & 97.5517 & 42.8645 \\
\hline $4 \mathrm{~B}$ & 46 & July $16 / 02$ & 0.5441 & 0.5124 & 0.7893 & 0.2236 & 43.6329 & 96.9737 & 44.9946 \\
\hline $1 \mathrm{~A}$ & 74 & Aug 13/02 & 0.5711 & 0.5059 & 0.8262 & 0.2335 & 46.1504 & 96.6918 & 47.7294 \\
\hline $1 \mathrm{~B}$ & 74 & Aug $13 / 02$ & 0.5735 & 0.5127 & 0.8652 & 0.2701 & 52.6770 & 97.5052 & 54.0248 \\
\hline $2 \mathrm{~A}$ & 74 & Aug 13/02 & 0.5726 & 0.5019 & 0.8036 & 0.2094 & 41.7165 & 98.0796 & 42.5333 \\
\hline $2 \mathrm{~B}$ & 74 & Aug 13/02 & 0.5866 & 0.5048 & 0.8134 & 0.2052 & 40.6448 & 98.2330 & 41.3759 \\
\hline $3 \mathrm{~A}$ & 74 & Aug 13/02 & 0.5833 & 0.5100 & 0.8054 & 0.2005 & 39.3088 & 98.4932 & 39.9102 \\
\hline $3 \mathrm{~B}$ & 74 & Aug 13/02 & 0.5806 & 0.5146 & 0.8076 & 0.2054 & 39.9096 & 98.3644 & 40.5733 \\
\hline $4 \mathrm{~A}$ & 74 & Aug 13/02 & 0.5871 & 0.5034 & 0.8821 & 0.2734 & 54.3057 & 97.8442 & 55.5022 \\
\hline $4 \mathrm{~B}$ & 74 & Aug 13/02 & 0.5906 & 0.5100 & 0.8806 & 0.2684 & 52.6225 & 98.6072 & 53.3658 \\
\hline $1 \mathrm{~A}$ & 109 & Sept 17/02 & 0.5933 & 0.5088 & 0.8393 & 0.2244 & 44.0989 & 98.3280 & 44.8487 \\
\hline $1 B$ & 109 & Sept 17/02 & 0.5735 & 0.5144 & 0.8509 & 0.2558 & 49.7230 & 98.8274 & 50.3129 \\
\hline $2 \mathrm{~A}$ & 109 & Sept 17/02 & 0.5829 & 0.5066 & 0.8180 & 0.2135 & 42.1388 & 98.9564 & 42.5832 \\
\hline $2 \mathrm{~B}$ & 109 & Sept 17/02 & 0.5893 & 0.5039 & 0.8411 & 0.2302 & 45.6787 & 99.3621 & 45.9720 \\
\hline $3 A$ & 109 & Sept 17/02 & 0.5519 & 0.5103 & 0.8254 & 0.2519 & 49.3582 & 98.9237 & 49.8952 \\
\hline $3 B$ & 109 & Sept 17/02 & 0.5696 & 0.5134 & 0.8014 & 0.2102 & 40.9379 & 99.8689 & 40.9916 \\
\hline $4 \mathrm{~A}$ & 109 & Sept 17/02 & 0.5871 & 0.5108 & 0.8713 & 0.2626 & 51.4047 & 99.2554 & 51.7903 \\
\hline $4 \mathrm{~B}$ & 109 & Sept 17/02 & 0.5684 & 0.5062 & 0.8411 & 0.2511 & 49.6000 & 98.8584 & 50.1727 \\
\hline $1 \mathrm{~A}$ & 123 & Oct 1/02 & 0.5168 & 0.5034 & 0.7841 & 0.2457 & 48.8031 & 97.7631 & 49.9198 \\
\hline $1 \mathrm{~B}$ & 123 & Oct $1 / 02$ & 0.5636 & 0.5083 & 0.8132 & 0.2280 & 44.8505 & 97.7269 & 45.8937 \\
\hline $2 A$ & 123 & Oct $1 / 02$ & 0.5831 & 0.5134 & 0.8106 & 0.2059 & 40.1003 & 98.3521 & 40.7722 \\
\hline $2 \mathrm{~B}$ & 123 & $\operatorname{Oct} 1 / 02$ & 0.5681 & 0.5023 & 0.8021 & 0.2124 & 42.2805 & 98.8792 & 42.7598 \\
\hline $3 A$ & 123 & Oct $1 / 02$ & 0.5755 & 0.5145 & 0.8261 & 0.2290 & 44.5044 & 99.1820 & 44.8714 \\
\hline $3 B$ & 123 & Oct $1 / 02$ & 0.5692 & 0.5094 & 0.8397 & 0.2489 & 48.8565 & 99.6255 & 49.0402 \\
\hline $4 A$ & 123 & Oct $1 / 02$ & 0.5701 & 0.5116 & 0.8499 & 0.2582 & 50.4642 & 98.8662 & 51.0430 \\
\hline $4 B$ & 123 & Oct $1 / 02$ & 0.5797 & 0.5125 & 0.8752 & 0.2739 & 53.4390 & 98.1267 & 54.4592 \\
\hline
\end{tabular}


Phase 2 - Acld Detergent Fibre (ADF) Determinatlon

Technician: Janice Haines, Soil Science

Dale: January 30,2003

Blank: 0.0216

\begin{tabular}{|c|c|c|c|c|c|c|c|c|c|}
\hline $\begin{array}{c}\text { Sample } \\
\text { Identitication }\end{array}$ & $\begin{array}{c}\text { Day of } \\
\text { Experiment }\end{array}$ & $\begin{array}{l}\text { Date of } \\
\text { Sample }\end{array}$ & Bag WI & Wet Wt & $\mathrm{Dry} W \mathrm{t}+\mathrm{Bag}$ & Dry Wt - Blank & $\%$ ADF & $\% \mathrm{DM}$ & $\begin{array}{l}\% \text { ADF (dry } \\
\text { matter basis) }\end{array}$ \\
\hline & & & (g) & (g) & (g) & (g) & & & \\
\hline $1 A$ & 137 & Oct $15 / 02$ & 0.5689 & 0.5014 & 0.7981 & 0.2076 & 41.3991 & 99.1152 & 41.7686 \\
\hline $1 \mathrm{~B}$ & 137 & Oct $15 / 02$ & 0.5522 & 0.5111 & 0.8155 & 0.2417 & 47.2853 & 99.6519 & 47.4505 \\
\hline $2 A$ & 137 & Oct $15 / 02$ & 0.5704 & 0.5043 & 0.8240 & 0.2320 & 45.9994 & 99.4153 & 46.2699 \\
\hline $2 \mathrm{~B}$ & 137 & Oct $15 / 02$ & 0.5303 & 0.5037 & 0.7890 & 0.2371 & 47.0667 & 99.8340 & 47.1450 \\
\hline $3 \mathrm{~A}$ & 137 & Oct 15/02 & 0.5713 & 0.5071 & 0.8138 & 0.2209 & 43.5565 & 99.6602 & 43.7050 \\
\hline $3 B$ & 137 & Oct $15 / 02$ & 0.5472 & 0.5017 & 0.7722 & 0.2034 & 40.5372 & 99.4816 & 40.7484 \\
\hline $4 \mathrm{~A}$ & 137 & Oct $15 / 02$ & 0.5329 & 0.5009 & 0.7821 & 0.2276 & 45.4332 & 99.1591 & 45.8185 \\
\hline $4 \mathrm{~B}$ & 137 & Oct $15 / 02$ & 0.5306 & 0.5029 & 0.7794 & 0.2272 & 45.1730 & 99.2010 & 45.5369 \\
\hline $1 \mathrm{~A}$ & 158 & Nov $5 / 02$ & 0.5814 & 0.5043 & 0.8567 & 0.2537 & 50.3024 & 98.6223 & 51.0051 \\
\hline $1 \mathrm{~B}$ & 158 & Nov 5/02 & 0.6008 & 0.5077 & 0.8674 & 0.2450 & 48.2519 & 99.5964 & 48.4475 \\
\hline $2 \mathrm{~A}$ & 158 & Nov $5 / 02$ & 0.5769 & 0.5049 & 0.8257 & 0.2272 & 44.9941 & 99.2790 & 45.3208 \\
\hline $2 B$ & 158 & Nov $5 / 02$ & 0.5876 & 0.5034 & 0.8370 & 0.2278 & 45.2473 & 99.3972 & 45.5217 \\
\hline $3 \mathrm{~A}$ & 158 & Nov $5 / 02$ & 0.5829 & 0.5030 & 0.8439 & 0.2394 & 47.5895 & 99.0157 & 48.0626 \\
\hline $3 B$ & 158 & Nov $5 / 02$ & 0.5870 & 0.5100 & 0.8170 & 0.2084 & 40.8578 & 99.3763 & 41.1143 \\
\hline $4 \mathrm{~A}$ & 158 & Nov $5 / 02$ & 0.5751 & 0.5075 & 0.8454 & 0.2487 & 49.0000 & 98.9877 & 49.5011 \\
\hline $4 \mathrm{~B}$ & 158 & Nov $5 / 02$ & 0.5606 & 0.5086 & 0.8248 & 0.2426 & 47.6947 & 98.7714 & 48.2879 \\
\hline $1 \mathrm{~A}$ & 180 & Nov $27 / 02$ & 0.5605 & 0.5087 & 0.8355 & 0.2534 & 49.8083 & 99.4830 & 50.0672 \\
\hline $1 B$ & 180 & Nov $27 / 02$ & 0.5917 & 0.5068 & 0.8563 & 0.2430 & 47.9430 & 99.3554 & 48.2540 \\
\hline $2 \mathrm{~A}$ & 180 & Nov $27 / 02$ & 0.5671 & 0.5000 & 0.8218 & 0.2331 & 46.6150 & 99.7112 & 46.7500 \\
\hline 2B & 180 & Nov $27 / 02$ & 0.5773 & 0.5021 & 0.8314 & 0.2325 & 46.3005 & 99.4983 & 46.5340 \\
\hline $3 A$ & 180 & Nov $27 / 02$ & 0.5653 & 0.5020 & 0.8208 & 0.2339 & 46.5886 & 99.7413 & 46.7095 \\
\hline $3 \mathrm{~B}$ & 180 & Nov $27 / 02$ & 0.5595 & 0.5011 & 0.8234 & 0.2423 & 48.3486 & 99.6358 & 48.5254 \\
\hline $4 \mathrm{~A}$ & 180 & Nov $27 / 02$ & 0.6009 & 0.5081 & 0.8785 & 0.2560 & 50.3789 & 99.6809 & 50.5402 \\
\hline $4 \mathrm{~B}$ & 180 & Nov $27 / 02$ & 0.5563 & 0.5091 & 0.8368 & 0.2589 & 50.8495 & 99.6525 & 51.0269 \\
\hline
\end{tabular}

\begin{tabular}{|c|c|c|c|c|c|c|c|}
\hline KWSTD & 0.5174 & 0.5079 & 0.6784 & 0.1394 & 27.4414 & 94.8831 & 28.9213 \\
\hline & 0.5233 & 0.5063 & 0.6850 & 0.1401 & 27.6664 & 94.8831 & 29.1584 \\
\cline { 2 - 8 } & 0.5856 & 0.5074 & 0.7602 & 0.1530 & 30.1488 & 94.8831 & 31.7747 \\
\cline { 2 - 8 } & 0.5899 & 0.5068 & 0.7470 & 0.1355 & 26.7315 & 94.8831 & 28.1730 \\
\hline
\end{tabular}

\begin{tabular}{|c|c|c|c|}
\hline Blank & 0.5224 & 0.5331 & 0.0107 \\
\hline & 0.5387 & 0.5619 & 0.0232 \\
\hline & 0.6031 & 0.6467 & 0.0436 \\
\hline & 0.5706 & 0.5796 & 0.0090 \\
\hline & \multicolumn{2}{|c|}{ Blank Mean $=$} & 0.0216 \\
\hline
\end{tabular}




\section{Scott Chapman-Results}

Technician: SC/JH

Date: Feb, 2002

\begin{tabular}{|c|c|c|c|c|c|c|c|c|}
\hline Samp & Day & Samp & $\%$ NDF & $\%$ ADF & $\%$ Lignin & $\%$ Cellulose & $\%$ Hemicell & $\% D M$ \\
\hline ID & No. & Date & DM Basis & DM Basis & DM Basis & DM Basis & DM Basis & \\
\hline $1 \mathrm{~A}$ & 18 & June $18 / 02$ & 73.6682 & 60.5729 & 15.6958 & 44.877098 & 13.095313 & 95.5945 \\
\hline $1 \mathrm{~B}$ & 18 & June 18/02 & 74.2072 & 56.8146 & 13.2472 & 43.567389 & 17.392557 & 96.5027 \\
\hline $2 A$ & 18 & June $18 / 02$ & 68.0195 & 66.7912 & 17.6170 & 49.174182 & 1.2283158 & 96.6968 \\
\hline $2 \mathrm{~B}$ & 18 & June 18/02 & 57.8379 & 48.8176 & 13.1455 & 35.672079 & 9.020261 & 96.3513 \\
\hline $3 A$ & 18 & June $18 / 02$ & 62.6861 & 47.2677 & 14.1692 & 33.098449 & 15.418461 & 95.6770 \\
\hline $3 B$ & 18 & June $18 / 02$ & 60.5044 & 48.2129 & 14.0835 & 34.129346 & 12.291521 & 97.4242 \\
\hline $4 \mathrm{~A}$ & 18 & June $18 / 02$ & 62.1295 & 46.9600 & 14.3528 & 32.60724 & 15.169474 & 97.3914 \\
\hline $4 \mathrm{~B}$ & 18 & June $18 / 02$ & 59.0591 & 48.3375 & 14.3345 & 34.003028 & 10.721566 & 97.1772 \\
\hline $1 \mathrm{~A}$ & 46 & July $16 / 02$ & 62.3106 & 59.5148 & 16.5053 & 43.00945 & 2.7958271 & 96.6796 \\
\hline $1 \mathrm{~B}$ & 46 & July $16 / 02$ & 63.9670 & 52.7459 & 15.1916 & 37.554368 & 11.22109 & 97.4057 \\
\hline $2 A$ & 46 & July $16 / 02$ & 6810 & 42.4439 & 14.1040 & 28.339921 & 10.237028 & 98.0005 \\
\hline $2 B$ & 46 & July $16 / 02$ & 46.8465 & 42.3351 & 15.4710 & 26.864124 & 4.511377 & 96.2310 \\
\hline $3 \mathrm{~A}$ & 46 & July $16 / 02$ & 52.2611 & 42.7084 & 15.0185 & 27.689925 & 9.5526939 & 96.3989 \\
\hline $3 \mathrm{~B}$ & 46 & July $16 / 02$ & 42.2934 & 34.1511 & 13.0099 & 21.141224 & 8.1423065 & 97.4163 \\
\hline $4 \mathrm{~A}$ & 46 & July $16 / 02$ & 50.8598 & 42.8645 & 15.2737 & 27.590733 & 7.9953006 & 97.5517 \\
\hline $4 \mathrm{~B}$ & 46 & July $16 / 02$ & 53.2821 & 44.9946 & 15.8610 & 29.133625 & 8.2874993 & 96.9737 \\
\hline $1 \mathrm{~A}$ & 74 & Aug $13 / 02$ & 2045 & 47.7294 & 17.1932 & 30.536255 & 12.475077 & 96.6918 \\
\hline $1 B$ & 74 & Aug 13/02 & 249 & 54.0248 & 17.6036 & 36.421241 & 4.2000562 & 97.5052 \\
\hline $2 \mathrm{~A}$ & 74 & Aug 13/02 & 384 & 42.5333 & 14.7487 & 27.784577 & 5.6051223 & 98.0796 \\
\hline $2 B$ & 74 & Aug 13/02 & 7445 & 41.3759 & 17.0399 & 24.336047 & 2.3685376 & 98.2330 \\
\hline $3 A$ & 74 & Aug 13/02 & 38.8983 & 39.9102 & 15.1296 & 30553 & -1.011868 & 98.4932 \\
\hline $3 B$ & 74 & Aug 13/02 & 102 & 40.5733 & 15.7847 & 24.788577 & 0.4369599 & 98.3644 \\
\hline $4 \mathrm{~A}$ & 74 & Aug $13 / 02$ & 8100 & 55.5022 & 22.0074 & 33.494777 & -3.692248 & 97.8442 \\
\hline $4 B$ & 74 & Aug 13/02 & 55.4447 & 53.3658 & 20.6993 & 32.666564 & 2.0788433 & 98.6072 \\
\hline $1 \mathrm{~A}$ & 109 & Sept 17/02 & 42.4308 & 44.8487 & 17.1290 & 27.719765 & -2.417961 & 98.3280 \\
\hline $1 \mathrm{~B}$ & 109 & Sept 17/02 & 51.5814 & 50.3129 & 16.3 & 33.926798 & 1.2684576 & 98.8274 \\
\hline $2 A$ & 109 & Sept $17 / 02$ & 3724 & 42.5832 & 16.0576 & 5608 & -4.21078 & 98.9564 \\
\hline $2 \mathrm{~B}$ & 109 & Sept $17 / 02$ & 35.3585 & 45.9720 & 16.8761 & 29.095821 & -10.61344 & 99.3621 \\
\hline $3 \mathrm{~A}$ & 109 & Sept $17 / 02$ & 41.8589 & 49.8952 & 17.6521 & 32.243099 & -8.036319 & 98.9237 \\
\hline $3 B$ & 109 & Sept $17 / 02$ & 39.3868 & 40.9916 & 15.6230 & 25.368629 & -1.604789 & 99.8689 \\
\hline $4 \mathrm{~A}$ & 109 & Sept $17 / 02$ & 142 & 51.7903 & 21.2619 & 30.528385 & -3.17615 & 99.2554 \\
\hline $4 \mathrm{~B}$ & 109 & Sept $17 / 02$ & 54.4913 & 50.1727 & 21.2029 & 28.969863 & 4.318566 & 98.8584 \\
\hline $1 \mathrm{~A}$ & 123 & Oct $1 / 02$ & 46.4543 & 49.9198 & 18.5357 & 31.384111 & -3.465468 & 97.7631 \\
\hline $1 B$ & 123 & Oct $1 / 02$ & 51.6638 & 45.8937 & 16.6897 & 29.20402 & 5.7701088 & 97.7269 \\
\hline $2 A$ & 123 & Oct $1 / 02$ & 46.7958 & 40.7722 & 16.1799 & 24.592334 & 6.0235659 & 98.3521 \\
\hline $2 B$ & 123 & Oct $1 / 02$ & 45.7266 & 42.7598 & 15.1819 & 27.577906 & 2.9668736 & 98.8792 \\
\hline $3 \mathrm{~A}$ & 123 & Oct $1 / 02$ & 43.9664 & 44.8714 & 16.2850 & 28.586365 & -0.904961 & 99.1820 \\
\hline $3 B$ & 123 & Oct $1 / 02$ & 38.7614 & 49.0402 & 16.9073 & 32.132839 & -10.27879 & 99.6255 \\
\hline $4 \mathrm{~A}$ & 123 & Oct $1 / 02$ & 46.5797 & 51.0430 & 21.9263 & 29.116679 & -4.463249 & 98.8662 \\
\hline $4 \mathrm{~B}$ & 123 & Oct $1 / 02$ & 53.0439 & 54.4592 & 23.8815 & 30.57773 & -1.415311 & 98.1267 \\
\hline
\end{tabular}




\section{Scott Chapman-Results}

Technician: SC/JH

Date: Feb, 2002

\begin{tabular}{|c|c|c|c|c|c|c|c|c|}
\hline Samp & Day & Samp & $\%$ NDF & $\%$ ADF & \% Lignin & $\%$ Cellulose & $\%$ Hemicell & $\% \mathrm{DM}$ \\
\hline ID & No. & Date & DM Basis & DM Basis & DM Basis & DM Basis & DM Basis & \\
\hline $1 \mathrm{~A}$ & 137 & Oct $15 / 02$ & 39.6648 & 41.7686 & 16.5814 & 25.187261 & -2.103817 & 99.1152 \\
\hline $1 \mathrm{~B}$ & 137 & Oct $15 / 02$ & 40.9936 & 47.4505 & 17.9669 & 29.483541 & -6.45685 & 99.6519 \\
\hline $2 A$ & 137 & Oct $15 / 02$ & 47.6397 & 46.2699 & 17.1941 & 29.075825 & 1.3697423 & 99.4153 \\
\hline $2 \mathrm{~B}$ & 137 & Oct $15 / 02$ & 45.5824 & 47.1450 & 16.3895 & 30.755484 & -1.56257 & 99.8340 \\
\hline $3 \mathrm{~A}$ & 137 & Oct $15 / 02$ & 47.1700 & 43.7050 & 15.5137 & 28.191308 & 3.4649849 & 99.6602 \\
\hline $3 \mathrm{~B}$ & 137 & Oct $15 / 02$ & 41.2865 & 40.7484 & 14.1477 & 26.60072 & 0.5381083 & 99.4816 \\
\hline $4 \mathrm{~A}$ & 137 & Oct $15 / 02$ & 49.2038 & 45.8185 & 19.4520 & 26.366482 & 3.3852553 & 99.1591 \\
\hline $4 \mathrm{~B}$ & 137 & Oct $15 / 02$ & 51.8831 & 45.5369 & 20.5493 & 24.987549 & 6.3462436 & 99.2010 \\
\hline $1 \mathrm{~A}$ & 158 & Nov $5 / 02$ & 37.8090 & 51.0051 & 18.1158 & 32.889328 & -13.19606 & 98.6223 \\
\hline $1 \mathrm{~B}$ & 158 & Nov $5 / 02$ & 42.0024 & 48.4475 & 17.9358 & 30.511676 & -6.445028 & 99.5964 \\
\hline $2 \mathrm{~A}$ & 158 & Nov $5 / 02$ & 36.7969 & 45.3208 & 15.4811 & 29.839679 & -8.52393 & 99.2790 \\
\hline $2 \mathrm{~B}$ & 158 & Nov $5 / 02$ & 40.3688 & 45.5217 & 15.8878 & 29.633921 & -5.152862 & 99.3972 \\
\hline $3 \mathrm{~A}$ & 158 & Nov $5 / 02$ & 40.4816 & 48.0626 & 15.9620 & 32.100537 & -7.580948 & 99.0157 \\
\hline $3 \mathrm{~B}$ & 158 & Nov $5 / 02$ & 36.7986 & 41.1143 & 14.2057 & \begin{tabular}{|l}
26.908562 \\
\end{tabular} & -4.315688 & 99.3763 \\
\hline $4 \mathrm{~A}$ & 158 & Nov $5 / 02$ & 45.9284 & 49.5011 & 19.8067 & 29.694427 & -3.572675 & 98.9877 \\
\hline $4 \mathrm{~B}$ & 158 & Nov $5 / 02$ & 47.1734 & 48.2879 & 19.1910 & 29.0969 & -1.114502 & 98.7714 \\
\hline $1 \mathrm{~A}$ & 180 & Nov $27 / 02$ & 43.6319 & 50.0672 & 19.3266 & 30.740519 & -6.435227 & 99.4830 \\
\hline $1 \mathrm{~B}$ & 180 & Nov $27 / 02$ & 41.8345 & 48.2540 & 19.1240 & 29.130042 & -6.419516 & 99.3554 \\
\hline $2 \mathrm{~A}$ & 180 & Nov $27 / 02$ & 43.9680 & 46.7500 & 16.7692 & 29.980753 & -2.782026 & 99.7112 \\
\hline $2 \mathrm{~B}$ & 180 & Nov $27 / 02$ & 44.0694 & 46.5340 & 17.2145 & 29.319463 & -2.464633 & 99.4983 \\
\hline $3 \mathrm{~A}$ & 180 & Nov $27 / 02$ & 43.3183 & 46.7095 & 16.7574 & 29.952054 & -3.391222 & 99.7413 \\
\hline $3 \mathrm{~B}$ & 180 & Nov $27 / 02$ & 40.8135 & 48.5254 & 16.8257 & 31.699627 & -7.711912 & 99.6358 \\
\hline $4 \mathrm{~A}$ & 180 & Nov $27 / 02$ & 45.7876 & 50.5402 & 20.8878 & 29.652317 & -4.752599 & 99.6809 \\
\hline $4 \mathrm{~B}$ & 180 & Nov $27 / 02$ & 45.7240 & 51.0269 & 21.7822 & 29.244668 & -5.302889 & 99.6525 \\
\hline KWSTD & & KWSTD & 36.627325 & 29.5068 & 5.0521 & 24.454786 & 7.4356712 & 94.8831 \\
\hline
\end{tabular}

